\title{
Generalized Seiberg-Witten and the Nahm Transform
}

\author{
DiSSERTATION \\ zur Erlangung des mathematisch-naturwissenschaftlichen Doktorgrades \\ Doctor rerum naturalium \\ der Georg-August-Universität Göttingen
}

im Promotionsprogramm Mathematical Sciences

der Georg-August University School of Science (GAUSS)

vorgelegt von

Robin Raymond

aus Göppingen

Göttingen, 2017 


\section{Betreuungsausschuss}

Prof. Dr. Victor Pidstrygach, Mathematisches Institut

Prof. Dr. Thomas Schick, Mathematisches Institut

Mitglieder der Prüfungskommission

Referent: Prof. Dr. Victor Pidstrygach, Mathematisches Institut

Korreferent: Prof. Dr. Thomas Schick, Mathematisches Institut

Weitere Mitglieder der Prüfungskommission

Prof. Dr. Karl-Henning Rehren, Institut für theoretische Physik

Prof. Dr. Henrik Seppänen, Mathematisches Intitut

Prof. Dr. Max Wardetzky, Intitut für Numerische und Angewandte Mathematik

Prof. Dr. Chengchang Zhu, Mathematisches Institut

Tag der mündlichen Prüfung: 24.01.2018 


\begin{abstract}
Using the viewpoint of principal bundles on hyperkähler reductions, we recover the results of Gocho and Nakajima [GN92] and give insights into the role that the quaternions play. We define a framework for dimensional reduction of gauge theories and show that the Haydys-Witten equations are dimensionally reduced Spin(7)-instantons. We extend the Nahm transform to data close to a solution satisfying the ordinary boundary conditions. Using generalized Seiberg-Witten, we show that $\mathbf{G}_{\mathbf{2}}$-Monopoles on $\Lambda_{+}^{2} \mathrm{X}$ and solutions of the Haydys-Witten equations on $\mathbb{R} \times X$ for $\mathrm{X}$ an oriented Riemannian 4-manifold are related to solutions of generalized Seiber-Witten equations with target the moduli space of Bogomolny monopoles and Nahm equations respectively. Applying the Nahm transform we derive a relation between $\mathbf{G}_{\mathbf{2}}$-Monopoles and solutions of the Haydys-Witten equations. Finally we hint how this can be extended via the extended Nahm transform.
\end{abstract}




\section{Acknowledgement}

Let me take the time to thank everyone who has supported me during the creation of this work. First and foremost I'd like to thank my supervisor Victor Pidstrygach for introducing me into the field and patiently answering my countless questions. I'd also like to thank my co-supervisor Thomas Schick for his support.

I'm very greatful for the financial support by the Research Training Group 1493 "Mathematical Structures in Modern Quantum Physics".

Furthermore I'd like to thank the attendees of the Tea Seminar for all the inspiring discussions throughout the years, specifically to the attendees Nuno Romão, Martin Callies, Ilias Tergiakidis and Felix Lubbe. I'm very thankful to Florian Beck for proofreading a draft of this work and to Florian Skorzinski for demystifying PDE.

Last but not least I'm in dept to my family and my girlfriend Jana Steffen for all the support I have received over the years.

"... just 3 more months ..." 


\section{Contents}

\begin{tabular}{llr}
\hline Introduction & 8
\end{tabular}

\begin{tabular}{|lll}
2 & Background Material & 10
\end{tabular}

$2.1 \quad$ Algebraic Structures . . . . . . . . . . . . . . . . . . . . . 10

2.1.1 Normed Division Algebras . . . . . . . . . . . . . . . . . 10

$2.1 .2 \quad$ Linear Algebra . . . . . . . . . . . . . . . . . . . 12

2.1 .3 Groups $\ldots \ldots \ldots \ldots \ldots \ldots \ldots$. . . . . . . . . . . . . . . . . . . . . . . . . . . . . . . . .

2.1 .4 Representations . . . . . . . . . . . . . . . . . . . . . . . . . . . . . . .

2.2 Manifolds of Special Holonomy . . . . . . . . . . . . . . . . . . 23

2.2.1 Hyperkähler structure . . . . . . . . . . . . . . . . 23

2.2 .2 Hypo structure $\ldots \ldots \ldots \ldots \ldots \ldots$

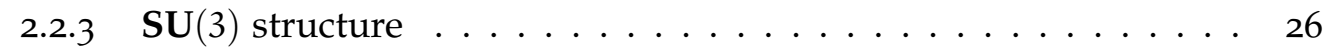

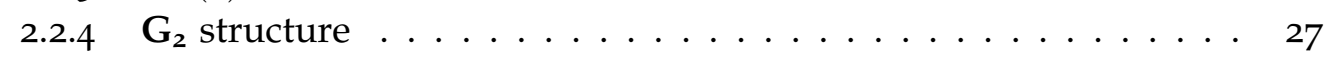

$2.2 .5 \quad \operatorname{Spin}(7)$ structure $\ldots \ldots \ldots \ldots \ldots \ldots . \ldots \ldots 28$

2.2 .6 Connection of Holonomies . . . . . . . . . . . . . . . . . 29

3 Hyperkähler Reduction 3 31

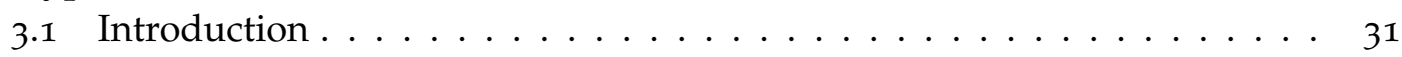

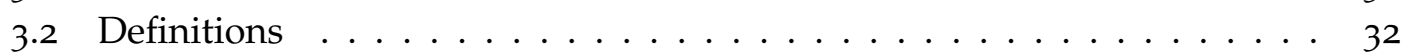

3.2.1 Reduction and Extensions . . . . . . . . . . . . . 34

3.2.2 The Correspondence of Forms … . . . . . . . . . . 35

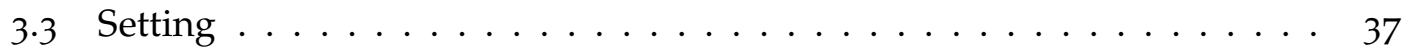

3.3.1 The Splitting of TM . . . . . . . . . . . . . . . . . . 37

3.3 .2 The Principal Bundles . . . . . . . . . . . . . . . . . . . 38

3.4 The Induced Connections $\ldots \ldots \ldots \ldots \ldots \ldots . \ldots \ldots . \ldots \ldots$

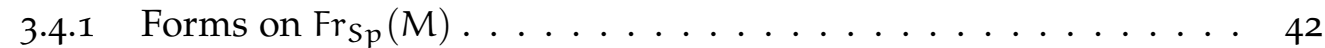

3.4 .2 Forms on $\mathrm{t}^{*} \mathrm{Fr}_{\mathrm{SO}}(\mathrm{M})$ and $\mathrm{t}^{*} \mathrm{Fr}_{\mathrm{Sp}}(\mathrm{M}) \ldots \ldots \ldots \ldots \ldots 4^{2}$

3.4 .3 Forms on $\operatorname{Fr}_{\mathrm{SO}}\left(\mu^{-1}(0), \mathrm{M}\right) \ldots \ldots \ldots \ldots \ldots . \ldots \ldots . \ldots \ldots 2$

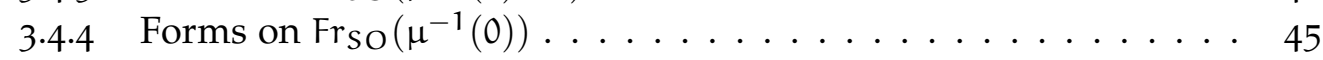

3.4 .5 Riemannian Submersions . . . . . . . . . . . . . . . . . . . . . . . . . . . .

3.4 .6 Forms on $\mathrm{Fr}_{\mathrm{SO}}\left(\mathrm{N}, \mu^{-1}(0)\right) \ldots \ldots \ldots \ldots \ldots . \ldots \ldots$ 
$3.4 \cdot 7$ Forms on $\mathrm{Fr}_{\mathrm{sp}}(\mathrm{N}, \mathrm{M}) \ldots \ldots \ldots \ldots \ldots \ldots \ldots \ldots$

3.5 Final Result $\ldots \ldots \ldots \ldots \ldots \ldots \ldots . \ldots \ldots \ldots$

3.5 .1 Preparation . . . . . . . . . . . . . . . . . . . 49

3.5 .2 The Results . . . . . . . . . . . . . . . 50

4 Gauge Theories 53

4.1 (Anti) Self-Duality Equations . . . . . . . . . . . . . . . . . 53

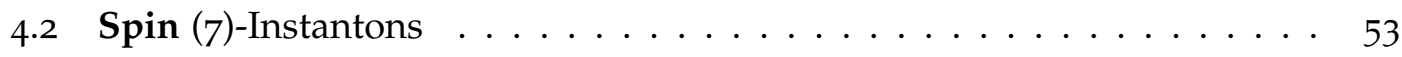

4.3 Generalized Seiberg-Witten Theory. . . . . . . . . . . . . . . . . 54

5 Dimensional Reduction $\quad \mathbf{5 6}$

5.1 Dimensional Reduction of a Gauge Theory . . . . . . . . . . . . . . . . . 56

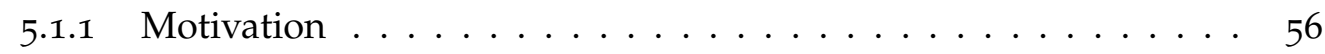

5.1 .2 Formulation $\ldots \ldots \ldots \ldots \ldots \ldots \ldots \ldots$

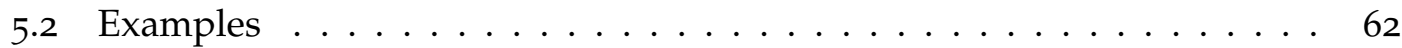

5.2.1 Bogomolny Equations . . . . . . . . . . . . . . . . . 63

5.2 .2 Hitchin System $\ldots \ldots \ldots$. . . . . . . . . . . . . . 63

5.2 .3 Nahm Equations . . . . . . . . . . . . . . . . . . . 64

5.2.4 Rotationally Invariant Nahm Equations. . . . . . . . . . . . . . . . . . 65

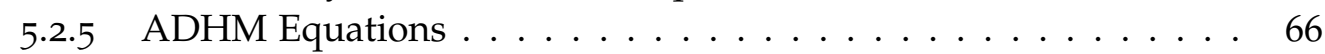

$5.2 .6 \quad \mathrm{G}_{2}$ Monopoles . . . . . . . . . . . . . . . . . . 66

5.2 .7 Haydys-Witten Equations . . . . . . . . . . . . . . . . . . 68

$\begin{array}{lll}6 & \text { Dimensionally Reduced Gauge Theories } & 75\end{array}$

6.1 Nahm Equations . . . . . . . . . . . . . . . . . . . . . . . . . . . . . . . . . . . . . 75

6.1.1 The Equations . . . . . . . . . . . . . . . . . 75

6.1 .2 Boundary Conditions. . . . . . . . . . . . . . . . . . 77

6.1 .3 The Moduli Space . . . . . . . . . . . . . . . . . . . . . . . . . 78

6.1 .4 Actions on the Moduli Space . . . . . . . . . . . . . . . . . 81

6.1 .5 As a Hyperkähler Reduction . . . . . . . . . . . . . . . . . . . . . 84

6.2 Bogomolny Equations $\ldots \ldots \ldots \ldots \ldots \ldots \ldots$

6.2 .1 The Equations $\ldots \ldots \ldots \ldots \ldots \ldots \ldots \ldots$

6.2 .2 Boundary Conditions . . . . . . . . . . . . . . . . 86

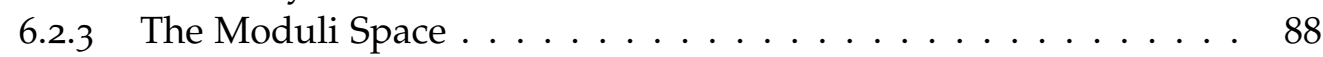

6.2 .4 Actions on the Moduli Space . . . . . . . . . . . . . . . . . . . . 91

6.2.5 As a Hyperkähler Reduction . . . . . . . . . . . . . . . . . . . . . . . . . . . . . . . . .

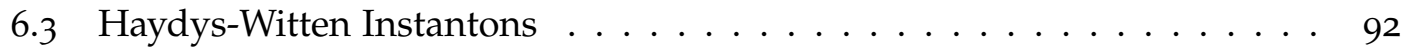

$6.4 \mathbf{G}_{\mathbf{2}}$-Monopoles $\ldots \ldots \ldots \ldots \ldots \ldots \ldots \ldots$ 
7 An Extended Nahm Transform 95

7.1 Notions . . . . . . . . . . . . . . . . . . . . . 96

7.2 An Extension of the Nahm Transform . . . . . . . . . . . . . . . . . 96

7.2.1 Nahm to Bogomolny . . . . . . . . . . . . . . . . . . . 97

7.2 .2 Bogomolny to Nahm . . . . . . . . . . . . . . . 100

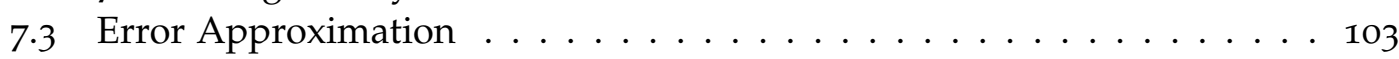

7.3.1 Nahm to Bogomolny . . . . . . . . . . . . . . . . . . . 103

$7 \cdot 3.2$ Bogomolny to Nahm . . . . . . . . . . . . . . . 106

7.4 Boundary Conditions . . . . . . . . . . . . . . . . . . 108

7.4.1 Nahm to Bogomolny . . . . . . . . . . . . . . . . . . . . 109

7.4 .2 Bogomolny to $\mathrm{Nahm} \ldots \ldots \ldots \ldots$. . . . . . . . . . . . . . . . .

7.5 Behavior with Respect to Conformal Maps . . . . . . . . . . . . . . 113

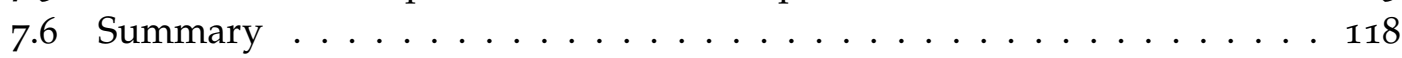

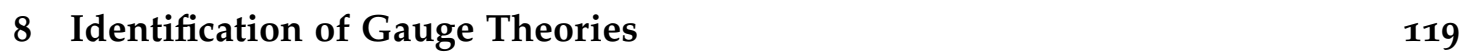

8.1 Prelude . . . . . . . . . . . . . . . . . . . . . . . . 119

8.2 General Construction . . . . . . . . . . . . . . . . . . . . . . . . 122

8.2.1 Reduction of Gauge Theories on Vector Bundles . . . . . . . . 122

8.2.2 Compensation through bigger Structure Groups . . . . . . . . . . 125

8.3 Examples . . . . . . . . . . . . . . . . . . . . . . 129

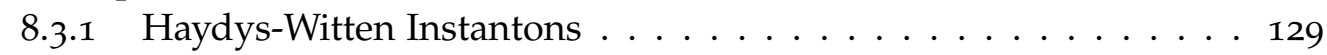

8.3 .2 Construction of Examples . . . . . . . . . . . . . . . . 135

$8.3 .3 \quad \mathrm{G}_{2}$ Monopoles . . . . . . . . . . . . . . . . . . . . . 136

8.3 .4 Construction of Examples . . . . . . . . . . . . . . . . . . 143

8.4 Adiabatic scaling . . . . . . . . . . . . . . . . . . . . . . . . . . . . . . . . . 144

8.5 A Transform Between $\mathbf{G}_{\mathbf{2}}$-Monopoles and solutions of the Haydys-Witten Equations ......................... 144

$\begin{array}{llr}9 & \text { Outlook } & 147\end{array}$

9.1 About the Connections . . . . . . . . . . . . . . . . . . . . . . 147

$9.2 \quad$ About the Boundary Conditions and Other Remarks . . . . . . . . . . 147

\begin{tabular}{lr}
\hline Bibliography & 149
\end{tabular} 


\section{Chapter 1}

\section{Introduction}

The origin of gauge theories lies in the Maxwell equations [Max65], the foundation of classical electromagnetism. Changing the structure group to the non-abelian group SU(2), we arrive at the anti self-duality equations which allowed Donaldson to make his groundbreaking advances in the theory of smooth structures of 4-manifolds [Don83].

Equations of these types can be derived on a larger class of manifolds if we restrict the holonomy of the underlying space. Similar to the anti self-duality equations, or Instanton equations as they are sometimes called in physics, we can define interesting gauge theoretic equations on manifolds with holonomy in e.g. $\mathbf{S O}(4), \mathbf{S U}(3), \mathbf{G}_{2}$, and $\operatorname{Spin}(7)$ [DS11a; Hay15b].

A different branch of gauge theory developed with the discovery of the SeibergWitten equations [SW94b; SW94a], which trade a more complicated equation for a simpler (abelian) gauge group $\mathbf{U}(1)$. It turned out that many of the results of the anti self-duality equations are also obtainable by these equations, and often much simpler to obtain.

Rather than just a connection, the Seiber-Witten equations accommodate a connection and a spinor, a section of the spin bundle. Generalized Seiberg-Witten theory replaces the spin bundle by a more complicated fiber bundle and allows to unify many gauge theories under this construction [Tau99: Pido4]. The typical fiber of these bundles has to be a hyperkähler manifold with a rotating $\mathbf{S p}(1)$-action. It turns out that the solution spaces of certain gauge theoretic equations have just these properties [Hay15a].

In Chapter 2 we introduce the necessary background material to understand the following chapters. The following Chapter 3 we investigate hyperähler reductions. These will play an important role in the final construction. Out approach is to work on the involved principal bundles, which allows us to recover the results of Gocho and Nakajima [GN92] and also a small novelty. The approach (which is very different to the one in $\left[\overline{\mathrm{GN}_{92}} \mid\right.$ ) also highlights the role the quaternions play in the construction.

Chapter 4 introduces the gauge theories that we will use in the rest of this work. In Chapter 5 we give a framework for dimensional reductions of gauge theories and 
apply this to construct all the well known reductions of the anti self-duality equations and Spin(7)-instantons. As a new result we show that the Haydys-Witten equations are a dimensional reduction of Spin(7)-instantons. We discuss some of the reduced equations in Chapter 6, with the focus being the Nahm equations and Bogomolny equations.

The Nahm transform relates solutions of the Nahm equations and Bogomolny equations, and we will recall the construction and show that it can be extended to a small neighborhood of the solution space of the equations in Chapter 7 In Chapter 8 we will use generalized Seiberg-Witten to relate solutions of the Haydys-Witten equations to solutions of the Nahm equations and similarly solutions of the $\mathbf{G}_{\mathbf{2}}$-Monopole equations to solutions of the Bogomolny equations. Combining this with the results of Chapter 7 allows is to define a map that relates $\mathbf{G}_{\mathbf{2}}$-Monopoles to solutions of the Haydys-Witten equations.

The last chapter, Chapter 9, briefly discusses how our results could be extended to a larger class of solutions and some interesting differences to the classical Nahm transform. Finally we mention interesting further research opportunities. 


\section{Chapter 2}

\section{Background Material}

\subsection{Algebraic Structures}

\subsubsection{Normed Division Algebras}

Definition 2.1 (Quaternions). Let $\mathbb{H}$ be the 4-dimensional real vector space spanned by $1, i, j, k$. $\mathbb{H}$ is the skew-field of quaternions if we define the multiplication via

$$
i^{2}=j^{2}=k^{2}=i j k=-1 .
$$

Definition 2.2 (Quaternionic Vectorspace). A vector space $V$ over the skew-field $\mathbb{H}$ is called a quaternionic vector space.

Remark 2.3 (Complex Structures). An equivalent way of defining a quaternionic vector space is by requiring a real vector space $V$ to carry a complex structure $I$ and an anti-linear real structure J,

$$
\mathrm{I}, \mathrm{J} \in \operatorname{End}(\mathrm{V}), \quad \mathrm{I}^{2}=-1, \quad \mathrm{~J}^{2}=1, \quad \mathrm{IJ}=-\mathrm{J} \mathrm{I} .
$$

Definition 2.4 (Octonions). Let $\mathrm{O}$ to be the 8-dimensional real vector space spanned by the basis

$$
\left\{1, e_{1}, e_{2}, \ldots, e_{7}\right\},
$$

and furnish it with a bilinear multiplication $\mathrm{O} \otimes_{\mathbb{R}} \mathrm{O} \rightarrow \mathrm{O}$ defined by the following diagram 


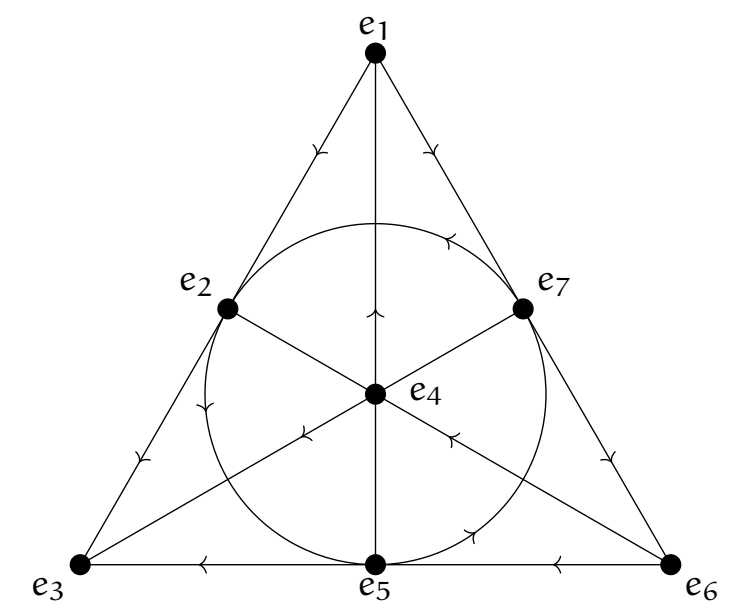

in the sense that for every oriented triple $\left(e_{i}, e_{j}, e_{k}\right)$ lying on an oriented curve, we require the quaternionic relations to hold,

$$
e_{i}^{2}=e_{j}^{2}=e_{k}^{2}=e_{i} e_{j} e_{k}=-1 .
$$

We call the division algebra $\mathrm{O}$ the octonions. Given an octonion $a=a_{0} 1+\sum_{i=1}^{7} a_{i} e_{i} \in \mathbb{O}$, we write

$$
\operatorname{Re}(a)=a_{0} 1, \quad \operatorname{Im}(a)=\sum_{i=1}^{7} a_{i} e_{i}, \quad \bar{a}=\operatorname{Re}(a)-\operatorname{Im}(a) .
$$

Remark 2.5. There are many different (but equivalent) ways to define the octonionic multiplication by distributing the symbols $e_{i}$ on the triangle above (in a consistent way). Our choice is not the most common, but it is essential that we make this very choice for chapter 5.2 .7 , 8.3.1 and 8.3.3. It is not completely clear to the author where this freedom of choice has its origin. However chapter 8.3.1 and 8.3.3 show that it is closely related to the choice of self-dual vs. anti self-dual connections.

Remark 2.6. - The standard scalar product on $\mathbb{R}^{8}$ can be described via octonions by

$$
\langle a, b\rangle_{\mathbb{R}^{8}}=\operatorname{Re}(a \bar{b}), \quad a, b \in \mathrm{O} .
$$

- The octonions $\mathrm{O}$ are a normed division algebra but are neither commutative nor associative. The former is no surprise, since we see that we have copies of $\mathbb{H}$ laying in $\mathrm{O}$, e.g. for every directed line in the diagram above. To see the latter, pick three $e_{i}$ not living in the same copy of $\mathbb{H}$, e.g.

$$
\left(e_{1} e_{3}\right) e_{6}=-e_{2} e_{6}=-e_{4} \neq e_{4}=-e_{1} e_{5}=e_{1}\left(e_{3} e_{6}\right) .
$$




\subsubsection{Linear Algebra}

In this section we will define some notation and prove a few easy facts that are hard to find a reference for.

Notation 2.7. Let $M$ be a manifold and $G \frown M$ a Lie group acting on $M$. For $\xi \in \mathfrak{g}$ and $x \in M$, we denote by

$$
K^{\xi} \in \Gamma(T M), \quad K_{x}^{\xi}=\left.\frac{d}{d t}\right|_{t=0}(\exp (t \xi) x),
$$

the corresponding fundamental vector fields.

Definition 2.8. Let $M$ be a manifold and $G \frown M$ a group acting on $M$. Let $\omega \in$ $\Omega^{k}(M, V)$ be a form with values in some vector bundle $V \rightarrow M$. We denote by

$$
\iota_{\mathfrak{g}} \omega \in \mathfrak{g}^{\vee} \otimes_{\mathbb{R}} \Omega^{k-1}(M, V),
$$

the form defined by $\left\langle\mathfrak{t}_{\mathfrak{g}} \omega, \xi\right\rangle=\mathfrak{t}_{\mathfrak{K} \xi} \omega$ for $\xi \in \mathfrak{g}$.

Lemma 2.9. Let $\mathrm{V}$ be an $\mathrm{n}$-dimensional oriented euclidean vector space and $\mathrm{V}=\mathrm{U} \oplus \mathrm{W}$ as an oriented orthogonal sum, where $\operatorname{dim} \mathrm{U}=\mathrm{m}$. Let $\mathrm{p}, \mathrm{q} \in \mathbb{N}_{0}$ such that $\mathrm{p}+\mathrm{q} \in\{0, \ldots, \mathrm{n}\}$. Then we may express the Hodge operator $\star_{\mathrm{V}}$ via $\star \mathrm{u}$ and $\star \mathrm{W}$ with respect to the bigrading of $\wedge^{\mathrm{p}+\mathrm{q}} \mathrm{V}$ as follows

$$
\left.\star v\right|_{\wedge p, q}=\left.\left.(-1)^{(m-p) q} \star u\right|_{\Lambda^{p}} \otimes \star w\right|_{\wedge q} .
$$

Proof. Let $e_{1}, \ldots, e_{m}$ be an orthonormal and oriented basis of $U$, and $e_{m+1}, \ldots, e_{n}$ of $W$. A basis of $\wedge^{p, q} \vee$ is given by $e_{I} \wedge e_{J}$ where $I \subset\{1, \ldots, m\},|I|=p$ and $J \subset\{m+1, \ldots, n\}$, $|J|=q$, where we define for $I=\left\{i_{1}, \ldots, i_{p}\right\}$

$$
e_{\mathrm{I}}=e_{i_{1}} \wedge \ldots \wedge e_{i_{p}}
$$

We calculate

$$
\begin{aligned}
\star_{\mathrm{V}}\left(e_{\mathrm{I}} \wedge e_{\mathrm{J}}\right) & =\operatorname{sgn}\left(\mathrm{I}, \mathrm{J}, \mathrm{I}^{\mathrm{c}}, \mathrm{J}^{\mathrm{c}}\right) e_{\mathrm{I}^{\mathrm{c}}} \wedge e_{\mathrm{J}^{\mathrm{c}}} \\
\left(\star_{\mathrm{U}} \otimes{ }^{\star} \mathrm{W}\right)\left(e_{\mathrm{I}} \wedge e_{\mathrm{J}}\right) & =\operatorname{sgn}\left(\mathrm{I}, \mathrm{I}^{\mathrm{c}}\right) \operatorname{sgn}\left(J, \mathrm{~J}^{\mathrm{c}}\right) e_{\mathrm{I}^{\mathrm{c}}} \wedge e_{\mathrm{J}^{\mathrm{c}}},
\end{aligned}
$$

where $I^{c}$ is the complement of $I$ in $\{1, \ldots, m\}$ and similarly for $J$. If $I=\left\{i_{1}, \ldots, i_{p}\right\}$ and $I^{\mathfrak{c}}=\left\{i_{p+1}, \ldots i_{m}\right\}$, then $\left(I, I^{c}\right)$ denotes the permutation sending $k \rightarrow i_{k}$. Since $\left(\mathrm{I} \cup \mathrm{I}^{\mathrm{c}}\right) \cap\left(\mathrm{J} \cup \mathrm{J}^{\mathrm{c}}\right)=\varnothing$, we have

$$
\operatorname{sgn}\left(I, I^{c}\right) \operatorname{sgn}\left(J, J^{c}\right)=\operatorname{sgn}\left(I, I^{c}, J, J^{c}\right)=(-1)^{|J| \cdot\left|I^{c}\right|} \operatorname{sgn}\left(I, J, I^{c}, J^{c}\right),
$$

which shows the claim. 
Notation 2.10. Let $M$ be a oriented Riemannian manifold and $\omega \in \Omega(M)$ a form on $M$. We will be often looking at the maps

$$
\Omega(M) \rightarrow \Omega(M), \quad \eta \mapsto \omega \wedge \eta,
$$

and

$$
\Omega(M) \rightarrow \Omega(M), \quad \eta \mapsto \star(\omega \wedge \eta),
$$

so we fix the notation

$$
F(\omega): \Omega(M) \rightarrow \Omega(M), F(\omega)(\eta)=\omega \wedge \eta
$$

and

$$
\mathrm{G}(\omega): \Omega(M) \rightarrow \Omega(M), G(\omega)(\eta)=\star(\omega \wedge \eta) .
$$

Lemma 2.11. Let $(\mathrm{V}, \mathrm{i}, \mathrm{j}, \mathrm{k})$ and $(\mathrm{W}, \mathrm{I}, \mathrm{J}, \mathrm{K})$ be two quaternionic vector spaces. Let $\alpha: \mathrm{V} \rightarrow \mathrm{W}$ be a quaternionic linear and $\beta: \mathrm{V} \rightarrow \mathrm{W}$ be elementary quaternionic antilinear, i.e. there is $a$ $a \in\{i, j, k\}$ with

$$
\beta(a v)=A \beta(v), \quad \beta(b v)=-B \beta(v) \quad \forall v \in V, b \neq a .
$$

Fixing any one of the complex structures, we can decompose the forms into bidegrees,

$$
\Lambda^{k}(V) \cong \bigoplus_{p+q=k} \Lambda_{a}^{p} V \otimes \Lambda_{a}^{q} V
$$

Then

1. $\alpha$ respects this decomposition for all $\mathrm{a} \in\{i, j, k\}$,

2. $\beta$ respects this decomposition for $a$. For $b \neq a$ it swaps the indices, i.e. if $\eta \in \Lambda_{A}^{p, q} W$, then $\beta^{*} \eta \in \Lambda_{\mathrm{a}}^{\mathrm{q}, \mathrm{p}} \mathrm{V}$.

Proof. This follows immediately from an easy calculation.

Corollary 2.12. In the setting of the last Lemma, let $\gamma: \mathrm{V} \rightarrow \mathrm{W}$ be quaternionic antilinear, i.e. in the span of elementary quaternionic antilinearity for all three structures. Then $\gamma$ fixes $\Lambda_{\mathrm{a}}^{\mathrm{q}, \mathrm{q}}$ for all structures $a \in\{i, j, k\}$.

Lemma 2.13. Let $(\mathrm{V}, \mathrm{i}, \mathrm{j}, \mathrm{k})$ be a 4-dimensional quaternionic vector space. If $\eta \in \Lambda_{\mathrm{a}}^{1,1} \mathrm{~V}$ for all $a \in\{i, j, k\}$, then $\eta \in \Lambda_{-}^{2} V$

Proof. Note that $\Lambda_{-}^{2} \mathrm{~V} \otimes_{\mathbb{R}} \mathbb{C}$ is given by $\omega_{\mathrm{a}}^{\perp} \subset \Lambda_{\mathrm{a}}^{1,1} \mathrm{~V}$, i.e. it is the orthogonal complement of $\omega_{a}$ in $\Lambda_{a}^{1,1} V$. Since $\omega_{a} \perp \omega_{b}$ for $a \neq b$, and $\eta$ is in $\Lambda^{1,1} V$ for all complex structures $a$, the claim follows. 


\subsubsection{Groups}

In this section we will recall the definitions of groups that play an important role in this work. Furthermore, we will mention some connections between these groups and discuss characterizations that will become important later on.

Definition 2.14 $(\mathbf{O}(n), \mathbf{S O}(n), \mathbf{U}(n), \mathbf{S U}(n), \mathbf{S p}(n))$. As always, define the classical matrix groups as

$$
\begin{aligned}
\mathbf{O}(\mathrm{n}) & =\left\{A \in \mathbf{G l}(\mathrm{n}, \mathbb{R}) \mid A^{\mathrm{t} A}=\mathbb{1}\right\} & & \subset \mathbf{G l}(\mathrm{n}, \mathbb{R}) \\
\mathbf{S O}(\mathrm{n}) & =\{\mathrm{A} \in \mathbf{O}(\mathrm{n}) \mid \operatorname{det} A=1\} & & \subset \mathbf{O}(\mathrm{n}) \\
\mathbf{U}(\mathrm{n}) & =\left\{\mathrm{A} \in \mathbf{G l}(\mathrm{n}, \mathbb{C}) \mid A^{*} A=\mathbb{1}\right\} & & \subset \mathbf{G l}(\mathrm{n}, \mathbb{C}) \\
\mathbf{S U}(\mathrm{n}) & =\{A \in \mathbf{U}(\mathrm{n}) \mid \operatorname{det} A=1\} & & \subset \mathbf{U}(\mathrm{n}) \\
\mathbf{S p}(\mathrm{n}) & =\left\{\mathrm{A} \in \mathbf{G l}(\mathrm{n}, \mathbb{H}) \mid A^{*} A=\mathbb{1}\right\} & & \subset \mathbf{G l}(\mathrm{n}, \mathbb{H}) .
\end{aligned}
$$

Definition 2.15 $(\operatorname{Spin}(n))$. For $n>2$, denote by $\operatorname{Spin}(n)$ the universal cover of $\mathbf{S O}(n)$. Remark 2.16. We are particularly interested in Spin(7). The following description will become handy later on,

$$
\operatorname{Spin}(7)=\{A \in \mathbf{S O}(8) \mid \exists B \in \mathbf{S O}(7), \forall a, b \in \mathbb{O}:(B a)(A b)=A(a b)\},
$$

where $B$ acts on the imaginary part of the Octonions. The map to $\mathbf{S O}(7)$ is in this notation given by

$$
\mathrm{A} \mapsto \mathrm{B} .
$$

This uses the triality of $\mathbf{S O}(7)$, for a justification see [Yokog].

Definition 2.17 $\left(G_{2}\right)$. Define $G_{2}$ to be the octonionic automorphism group of $O$, that is the real automorphisms of $\mathrm{O}$ that respect the multiplication

$$
\mathbf{G}_{\mathbf{2}}=\operatorname{Aut}_{\mathbf{O}}(\mathbf{O})=\left\{\varphi: \operatorname{Aut}_{\mathbb{R}}(\mathbf{O}) \mid \varphi(\mathrm{a} \cdot \mathrm{b})=\varphi(\mathrm{a}) \cdot \varphi(\mathrm{b}), \forall \mathrm{a}, \mathrm{b} \in \mathbf{O}\right\} .
$$

Remark 2.18. We can realize these groups as subgroups of $\mathbf{S O}(n)$, for certain $n \in \mathbb{N}$. We are interested here in

$$
\begin{gathered}
\text { - } \iota_{1}: \mathbf{S U}(2) \hookrightarrow \mathbf{S O}(4), \quad\left(\begin{array}{ll}
a & b \\
c & d
\end{array}\right) \mapsto\left(\begin{array}{ll}
\mathfrak{l}(a) & \mathfrak{l}(b) \\
\mathfrak{l}(b) & \mathfrak{l}(a)
\end{array}\right), \text { where } \\
\mathfrak{l}(a)=\left(\begin{array}{cc}
\operatorname{Re}(a) & -\operatorname{Im}(a) \\
\operatorname{Im}(a) & \operatorname{Re}(a)
\end{array}\right), \\
\text { - } \iota_{2}: \mathbf{S U}(2) \hookrightarrow \mathbf{S O}(5), \quad A \mapsto\left(\begin{array}{cc}
1 & 0 \\
0 & \iota_{1} A
\end{array}\right),
\end{gathered}
$$


- $\iota_{3}: \mathbf{S U}(3) \hookrightarrow \mathbf{S O}(6)$, similar to $\iota_{1}$,

- $\iota_{4}: \mathbf{G}_{2} \subset \mathbf{S O}(7),\left.\quad \varphi \mapsto \operatorname{pr}_{\operatorname{ImO}} \circ \varphi\right|_{\operatorname{ImO} \text {. }}$

- $\iota_{5}: \operatorname{Spin}(7) \subset \mathbf{S O}(8)$, using (2.26).

For $\mathfrak{i}_{4}$, realize that the multiplicativity of $\mathbf{G}_{\mathbf{2}}$ implies that $\varphi(1)=1$ and via $\varphi(\bar{a})=\overline{\varphi(a)}$ we conclude that $\varphi$ is an isometry with respect to the standard scalar product on $\mathbb{R}^{8}$. Hence $\varphi \in \mathbf{O}(\operatorname{Im} \mathbf{O})$, and since $\mathbf{G}_{\mathbf{2}}$ is connected, even $\varphi \in \mathbf{S O}(\operatorname{Im} \mathbf{O})$.

Remark 2.19. $\quad$ Note that $\mathbf{S U}(2)=\mathbf{S p}(1)$ with some choice of $\mathbb{H} \cong \mathbb{C}^{2}$.

- We can give alternative characterizations of the above groups as stabilizers of certain objects. This is discussed in detail in Proposition 2.28.

Notation 2.20. For a manifold $M$ and a basis of one-forms $d x^{i}$ we use the notation

$$
d x^{i_{1} i_{2} \ldots i_{k}}=d x^{i_{1}} \wedge \ldots \wedge d x^{i_{k}} .
$$

If $M=\mathbb{R}^{k}$, then $e^{i}$ denote the canonical basis of one-forms.

Definition 2.21 (Hyperkähler structure forms). The following 2-forms on $\mathbb{R}^{4}$,

$$
\omega_{1}^{\prime}=e^{12}+e^{34}, \quad \omega_{2}^{\prime}=e^{13}-e^{24}, \quad \omega_{3}^{\prime}=e^{14}+e^{23},
$$

are called the hyperkähler structure forms.

Definition 2.22 (Hypo structure forms). The following forms on $\mathbb{R}^{5}$ are called the hypo structure forms.

$$
\eta^{\prime}=e^{1}, \quad \eta_{1}^{\prime}=e^{23}+e^{45}, \quad \eta_{2}^{\prime}=e^{24}-e^{35}, \quad \eta_{3}^{\prime}=e^{25}+e^{34} .
$$

Definition 2.23 (SU(3) structure forms). The following forms on $\mathbb{R}^{6}$

$$
\omega^{\prime}=-e^{12}+e^{34}+e^{56}, \quad \Omega^{\prime}=-e^{135}+e^{146}-e^{236}-e^{245},
$$

are called $\mathbf{S U}(3)$ structure forms.

Definition 2.24 ( $G_{2}$ structure form). The following form on $\mathbb{R}^{7}$

$$
\varphi^{\prime}=e^{123}-e^{145}-e^{167}-e^{246}+e^{257}-e^{347}-e^{356}
$$

is called the $\mathbf{G}_{\mathbf{2}}$-structure form.

Remark 2.25. Our choice of octonionic multiplication induces the signs in this structure form. 
Definition 2.26 (Spin(7) structure form). The following form on $\mathbb{R}^{8}$

$$
\begin{aligned}
\Theta^{\prime} & =e^{1234}-e^{1256}-e^{1278}-e^{1357}+e^{1368}-e^{1458}-e^{1467} \\
& -e^{2358}-e^{2367}+e^{2457}-e^{2468}-e^{3456}-e^{3478}+e^{5678}
\end{aligned}
$$

is the $\operatorname{Spin}(7)$-structure form.

Remark 2.27. Note that we have the following relation between these structure forms.

- If $\omega_{1}^{\prime}, \omega_{2}^{\prime}, \omega_{3}^{\prime}$ are hyperkähler structure forms on $\mathbb{R}^{4}$, then

$$
\eta^{\prime}=e^{0}, \quad \eta_{i}^{\prime}=\omega_{i}^{\prime}, \quad i \in\{1,2,3\} .
$$

form hypo structure forms on $\mathbb{R}^{5}=\mathbb{R} \oplus \mathbb{R}^{4}$.

- If $\eta^{\prime}, \eta_{i}^{\prime}$ are hypo structure forms on $\mathbb{R}^{5}$, then

$$
\begin{aligned}
& \omega^{\prime}=-e^{0} \wedge \eta^{\prime}+\eta_{1}^{\prime} \\
& \Omega^{\prime}=e^{0} \wedge \eta_{2}^{\prime}-\eta^{\prime} \wedge \eta_{3}^{\prime}
\end{aligned}
$$

are $\mathbf{S U}(3)$ structure forms of $\mathbb{R}^{6}=\mathbb{R} \oplus \mathbb{R}^{5}$. From Lemma 2.9

$$
\begin{aligned}
& \star_{6} \omega^{\prime}=-\star_{5} \eta^{\prime}+e^{0} \wedge \star_{5} \eta_{1}^{\prime} \\
& \star_{6} \Omega^{\prime}=\star_{5} \eta_{2}^{\prime}-e^{0} \wedge \star_{5}\left(\eta^{\prime} \wedge \eta_{3}^{\prime}\right) .
\end{aligned}
$$

- If $\omega^{\prime}, \Omega^{\prime}$ are $\mathbf{S U}(3)$ structure forms on $\mathbb{R}^{6}$, then

$$
\varphi^{\prime}=-e^{0} \wedge \omega^{\prime}+\Omega^{\prime}
$$

is a $\mathbf{G}_{\mathbf{2}}$ structure form on $\mathbb{R}^{7}=\mathbb{R} \oplus \mathbb{R}^{6}$. By Lemma 2.9 we also get

$$
\psi^{\prime}=\star_{7} \varphi^{\prime}=-\star_{6} \omega-e^{0} \wedge \star_{6} \Omega .
$$

- If $\varphi^{\prime}$ is the $\mathbf{G}_{\mathbf{2}}$ structure form on $\mathbb{R}^{7}$, then

$$
\Theta^{\prime}=e^{\mathcal{O}} \wedge \varphi^{\prime}+\star_{7} \varphi^{\prime}
$$

is the $\operatorname{Spin}(7)$ structure form. Note that $\star_{7}$ is induced by the metric arising from $\mathbf{G}_{\mathbf{2}} \subset \mathbf{S O}(7)$.

Proposition 2.28. We can characterize the groups above as follows

1. $\mathbf{S U}(2) \cong \mathbf{S O}(4) \cap \mathbf{S}\left(\omega_{1}^{\prime}\right) \cap \mathbf{S}\left(\omega_{2}^{\prime}\right) \cap \mathbf{S}\left(\omega_{3}^{\prime}\right)$

2. $\mathbf{S U}(2) \cong \mathbf{S O}(5) \cap \mathbf{S}\left(\eta^{\prime}\right) \cap \mathbf{S}\left(\eta_{1}^{\prime}\right) \cap \mathbf{S}\left(\eta_{2}^{\prime}\right) \cap \mathbf{S}\left(\eta_{3}^{\prime}\right)$

3. $\mathbf{S U}(3) \cong \mathbf{S O}(6) \cap \mathbf{S}\left(\omega^{\prime}\right) \cap \mathbf{S}\left(\Omega^{\prime}\right)$, 
4. $\mathbf{G}_{\mathbf{2}} \cong \mathbf{G l}(7) \cap \mathbf{S}\left(\varphi^{\prime}\right)$,

5. $\operatorname{Spin}(7) \cong \mathbf{G l}(8) \cap \mathbf{S}\left(\Theta^{\prime}\right)$.

where $\mathbf{S}(-)$ is the stabilizer of a form.

Proof. The proof of all these facts are similar.

Ad 1. Note that $\mathbf{S U}(2) \cong \mathbf{S p}(1)$ and the latter is the stabilizer of

$$
\langle-,-\rangle_{\mathbb{H}}: \mathbb{H} \times \mathbb{H} \rightarrow \mathbb{H}, \quad\langle x, y\rangle_{\mathbb{H}}=x \bar{y} .
$$

With the identification $\mathbb{H} \cong \mathbb{R}^{4}$ via $x_{0}+i x_{1}+j x_{2}+k x_{3} \leftrightarrow\left(x_{0}, x_{1}, x_{2}, x_{3}\right)$ (and $\mathrm{T}_{\mathrm{a}} \mathbb{R}^{4} \cong \mathbb{R}^{4}$ ) this implies after a short calculation that

$$
\langle x, y\rangle_{\mathbb{H}}=\langle x, y\rangle_{\mathbb{R}}-i \omega_{i}^{\prime}(x, y)-j \omega_{j}^{\prime}(x, y)-k \omega_{k}^{\prime}(x, y),
$$

is stabilized, where $\langle-,-\rangle_{\mathbb{R}}$ is the canonical scalar product on $\mathbb{R}^{4}$. This shows the claim.

Ad 2. Follows immediately from 1.

Ad 3. A similar argument as in 1 shows that the stabilizer of $\langle-,-\rangle_{\mathbb{C}}$ (which is $\mathbf{U}(3)$ ) is the intersection of $\mathbf{S O}(6)$ with the stabilizer of $\omega^{\prime}$. Stabilizing $\Omega^{\prime}$ is then equivalent to stabilizing the determinant $\Lambda^{3,0}$ form, which is given by $\Omega^{\prime}+J \Omega^{\prime}$, where $\mathrm{J}$ is the almost complex structure induced by $\mathbf{S O}(6)$ and $\omega^{\prime}$. This is equivalent to stabilizing $\Omega^{\prime}$.

Ad 4. The octonionic multiplication is given by

$$
a \cdot b=\langle a, b\rangle_{\mathbb{R}^{8}}+a \times b, \quad a, b \in \mathbb{O} .
$$

Furthermore $\varphi^{\prime}$ is the 3-form given by

$$
\varphi^{\prime}(x, y, z)=\langle x, y \times z\rangle,
$$

so stabilizing $\varphi^{\prime}$ is equivalent to stabilizing $\times$ in $\mathbf{S O}(7)$. But stabilizing $\varphi$ automatically implies stabilizing $\langle-,-\rangle$, because

$$
6\langle-,-\rangle \text { vol }=\iota_{-} \varphi \wedge \iota_{-} \varphi \wedge \varphi .
$$

Ad 5. See e.g. [Sal89, Lemma 12.2].

Corollary 2.29. We have the following relation between the groups.

1. $\operatorname{Spin}(7) \cap(\mathbf{S O}(1) \times \mathbf{S O}(7)) \cong \mathbf{G}_{2}$ 
2. $\mathbf{G}_{\mathbf{2}} \cap(\mathbf{S O}(1) \times \mathbf{S O}(6)) \cong \mathbf{S U}(3)$

3. $\mathbf{G}_{\mathbf{2}} \cap(\mathrm{SO}(3) \times \mathbf{S O}(4)) \cong \mathbf{S O}(4)$

4. $\mathrm{SU}(3) \cap(\mathrm{SO}(1) \times \mathrm{SO}(5)) \cong \mathbf{S U}(2)$

5. $\mathbf{S U}(2) \cap(\mathbf{S O}(1) \times \mathbf{S O}(4)) \cong \mathbf{S U}(2)$

where we embed the left most groups according to Remark 2.18

Proof. This follows immediately from Proposition 2.28 and Remark 2.27, except for 3 , which can be found in e.g. [Yokog].

\subsubsection{Representations}

To understand manifolds of special holonomies, we need to understand some of the representations of these groups. Luckily most of the groups we are interested in are simply connected, so representations of their Lie Algebras are in one-to-one correspondence with the representations of the groups. Unfortunately it is very hard to find references for representations of real Lie Algebras, so we need to introduce some notation.

Notation 2.30. Let all Lie Algebras be semi-simple, unless otherwise mentioned.

Definition 2.31 (Lie Algebra representation). A Lie Algebra representation of a Lie Algebra $\mathfrak{g}$ on a vector space $\mathrm{V}$ is a Lie algebra homomorphism

$$
\rho: \mathfrak{g} \rightarrow \mathfrak{g l}(\mathrm{V}) .
$$

- If $\mathfrak{g}$ is a real Lie Algebra and $\mathrm{V}$ a real vector space, we call the representation real.

- If $\mathfrak{g}$ is a real Lie Algebra, $\mathrm{V}$ is a complex vector space and $\rho: \mathfrak{g} \rightarrow \mathfrak{g l}_{\mathbb{C}}(\mathrm{V})$ is $\mathbb{R}$-linear, we call the representation complex.

- If $\mathfrak{g}$ is a real Lie Algebra, $V$ is a quaternionic vector space and $\rho: \mathfrak{g} \rightarrow \mathfrak{g l}_{\mathbb{H}}(\mathrm{V})$ is $\mathbb{R}$-linear, we call the representation quaternionic.

- If $\mathfrak{g}$ is a complex Lie Algebra, $\mathrm{V}$ is a complex vector space and $\rho: \mathfrak{g} \rightarrow \mathfrak{g l}_{\mathbb{C}}(\mathrm{V})$ is $\mathrm{C}$-linear, we call the representation complex linear.

- A representation of any of the types above is called irreducible if it has no nontrivial subrepresentations of the same type.

Remark 2.32. The theory of semi simple Lie Algebras generally works with complex Lie Algebras and complex linear representations. So there are three steps to identify these with representations of Lie Groups. 
1. Relate complex linear representations of the complexification of a Lie Algebra to complex representations of the underlying real Lie Algebra.

2. Relate complex representations to real representations.

3. Relate representations of Lie Algebras to representations of the corresponding Lie group.

We begin with $\mathrm{I}$.

Lemma 2.33. [Halo3, Proposition 4.6] Let $\mathfrak{g}$ be a real Lie Algebra. There is a $1-1$ correspondence of (finite dimensional) complex representations of $\mathfrak{g}$ and (finite dimensional) complex linear representations of $\mathfrak{g}^{\mathrm{C}}$. The correspondence respects irreducibility.

Proof. The correspondence is given by complex linear extension,

$$
x+i y \mapsto \rho(x)+i \rho(y),
$$

and restriction onto the real Lie Algebra.

For the second step of Remark 2.32 we use a theorem from Onishchik [Onio4]. Let us first introduce some notation.

Definition 2.34. - Let $\rho: \mathfrak{g} \rightarrow \mathfrak{g l}(\mathrm{V})$ be a real representation. Denote by $\rho^{\mathbb{C}}$ the induced complex representation of $\rho$ on $\mathbb{V}^{\mathbb{C}}=V \oplus i V$.

- Let $\rho: \mathfrak{g} \rightarrow \mathfrak{g l}(W)$ be a complex presentation. Then denote by $\rho_{\mathbb{R}}$ the real representation that is induced by forgetting the complex structure of $W$.

- Let $\rho: \mathfrak{g} \rightarrow \mathfrak{g l}(W)$ be a complex representation with invariant real structure. Then $\mathrm{W}=\mathrm{V}^{\mathrm{C}}$ for some real vector space $\mathrm{V}$ and $\rho$ induces a representation on $\mathrm{V}$. We denote this representation by $[W]$.

Proposition 2.35. [Onio4, Theorem §8.1]

Any irreducible real representation $\rho: \mathfrak{g} \rightarrow \mathfrak{g l}(\mathrm{V})$ of a real Lie algebra $\mathfrak{g}$ satisfies precisely one of the following two conditions:

1. $\rho^{\mathbb{C}}$ is an irreducible complex representation;

2. $\rho=\rho_{\mathbb{R}^{\prime}}^{\prime}$ where $\rho^{\prime}$ is an irreducible complex representation admitting no invariant real structures.

Conversely, any real representation $\rho$ satisfying 1 or 2 is irreducible.

Remark 2.36. We may turn this around and say that given any irreducible complex representation $\rho: \mathfrak{g} \rightarrow \mathfrak{g l}_{\mathbb{C}}(V)$, where $n=\operatorname{dim}_{\mathbb{C}} V$, one of two things can happen. Either it has an invariant real structure, which gives a irreducible representation of real 
dimension $n$; or it remains irreducible if we forget the complex structure, resulting in a an irreducible real representation of dimension $2 n$.

There are sophisticated techniques discussed in [Onio4] to see which of the two categories a representation belongs to. Being only interested in low dimensional examples, we will use an ad-hoc approach here.

Remark 2.37. The last step of Remark 2.32 concerns the first fundamental group. Again, there are more sophisticated methods to understand which representations factor through the projection, but we will only work with the most naive approach; a representation factors through if and only if the deck transformation group acts trivially on it.

On the other hand any (irreducible) representation of the quotient clearly induces a (irreducible) representation of the total space, so we get all possible representations.

\section{The representations of $\mathrm{SU}(2)$}

Because $\mathbf{S U}(2)$ is simply connected, the representations of $\mathbf{S U}(2)$ agree with the complex linear representations of $\mathfrak{s l}(2, \mathbb{C})$. These are characterized by the highest weight and given by symmetric powers of the tautological representation.

As $\mathfrak{s u}(2)$ representations, it follows immediately that $S^{i} \mathbb{C}^{2}$ has a real structure if and only if $i$ is even. This gives the following real representations

\begin{tabular}{cccc}
\hline Highest Weight & Name & Origin & Dimension \\
\hline $\mathrm{o}$ & $\mathbb{R}$ & {$\left[\mathrm{S}^{0} \mathbb{C}^{2}\right]$} & 1 \\
2 & $\mathfrak{s u}(2)$ & {$\left[\mathrm{S}^{2} \mathbb{C}^{2}\right]$} & 3 \\
1 & $\mathbb{C}_{\mathbb{R}}^{2}$ & $S^{1} \mathbb{C}_{\mathbb{R}}^{2}$ & 4 \\
4 & - & {$\left[S^{4} \mathbb{C}^{2}\right]$} & 5 \\
\hline
\end{tabular}

\section{Representations of $\mathrm{SO}(4)$}

We know that $\mathbf{S O}(4)$ has the double cover $\mathbf{S U}(2) \times \mathbf{S U}(2)$. The irreducible complex representations of $\mathbf{S U}(2)$ are given by $S^{i} \mathbb{C}^{2}$. Hence the irreducible complex representations of $\mathbf{S U}(2) \times \mathbf{S U}(2)$ are given by

$$
S^{i j}=S^{i} C^{2} \otimes S^{j} C^{2} .
$$

Figure 2.1: The Coxeter-Dynkin Diagram of $\mathfrak{s o}(4, \mathbb{C})$.

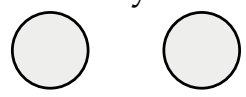

These representations factor through $\mathbf{S O}(4)$ if and only if $(-1,-1) \in \mathbf{S U}(2) \times \mathbf{S U}(2)$ acts trivially, which is true if either both $i$ and $j$ are even, or both are odd. If both $i$ and 
$j$ is even, then both components have a real structure, so also the tensor product. If both are odd, then both sides have a quaternionic structure and their tensor products give a real structure. Hence the irreducible real representations of $\mathbf{S O}(4)$ are given by

$$
\left\{\left[S^{i j}\right] \mid i+j \bmod 2=0\right\} .
$$

For notational simplicity, we will drop the $[-]$ and simply denote them by $S^{i j}$. The lowest dimensions are given by

\begin{tabular}{cccc}
\hline Highest Weight & Name & Origin & Dimension \\
\hline$(0,0)$ & $\mathbb{R}$ & $S^{00}$ & 1 \\
$(2,0)$ & $\Lambda_{+}^{2}$ & $S^{20}$ & 3 \\
$(0,2)$ & $\Lambda_{-}^{2}$ & $S^{02}$ & 3 \\
$(1,1)$ & $\mathbb{R}^{4}$ & $S^{11}$ & 4 \\
$(4,0)$ & - & $S^{40}$ & 5 \\
$(0,4)$ & - & $S^{04}$ & 5 \\
$(3,1)$ & - & $S^{31}$ & 8 \\
$(1,3)$ & - & $S^{13}$ & 8 \\
$(2,2)$ & - & $S^{22}$ & 9 \\
\hline
\end{tabular}

\section{Representations of SU(3)}

Again, $\mathbf{S U}(3)$ is simply connected. Actually all $\mathbf{S U}(n)$ are, by the fibration

$$
\mathrm{SU}(\mathrm{n}-1) \rightarrow \mathrm{SU}(\mathrm{n}) \rightarrow \mathrm{SU}(\mathrm{n}) / \mathrm{SU}(\mathrm{n}-1) \cong \mathrm{S}^{2 \mathrm{n}-1}
$$

So again, complex representations of $\mathbf{S U}(3)$ are equal to complex linear representations of $\mathfrak{s l}(3, \mathbb{C})$.

Figure 2.2: The Coxeter-Dynkin Diagram of $\mathfrak{s l}(3, \mathbb{C})$.

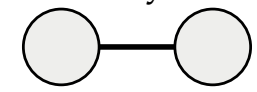

In this case we have a new phenomenon. Since we have an outer automorphism of the group (swapping the two nodes in Figure 2.2), we have two distinct irreducible representations of every dimension as long as the highest weight is not of type $(n, n)$, $n \in \mathbb{N}_{0}$. These representations are dual to each other, hence as representations of SU(3) they are conjugated. Since these do not possess a real structure, we forget the complex structure, which identifies these as real representations. 
Table 2.1: Some Irreducible Representations of SU(3)

\begin{tabular}{ccc}
\hline Highest Weight & Name & Dimension \\
\hline$(0,0)$ & $\mathbb{R}$ & 1 \\
$(1,0)$ or $(0,1)$ & $\mathbb{C}_{\mathbb{R}}^{3}$ & 6 \\
$(1,1)$ & $\mathfrak{s u}(3)$ & 8 \\
$(2,0)$ or $(0,2)$ & - & 12 \\
\hline
\end{tabular}

\section{The representations of $\mathrm{G}_{2}$}

The representations of $\mathbf{G}_{\mathbf{2}}$ are all equipped with an invariant real structure, and since $\mathbf{G}_{\mathbf{2}}$ is simply connected, representations are in correspondence with representations of $\mathfrak{g}_{2}^{\mathrm{C}}$.

Figure 2.3: The Coxeter-Dynkin Diagram of $\mathfrak{g}_{2}^{\mathbb{C}}$.

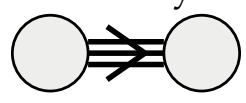

Table 2.2: Some Irreducible Real Representations of $\mathbf{G}_{\mathbf{2}}$.

\begin{tabular}{cccl}
\hline Highest Weight & Name & Dimension & Description \\
\hline$(0,0)$ & $\mathbb{R}$ & 1 & trivial representation \\
$(1,0)$ & $\mu$ & 7 & action of $\mathrm{G}_{2}$ on $\operatorname{Im}(\mathrm{O})$ \\
$(0,1)$ & $\mathfrak{g}_{2}$ & 14 & the adjoint representation \\
$(2,0)$ & $\mathrm{S}_{0}^{2} \mu$ & 27 & \\
$(1,1)$ & - & 64 & \\
$(3,0)$ & - & 77 & \\
$(0,2)$ & - & 77 & \\
\hline
\end{tabular}

Lemma 2.38. If we restrict the group to $\mathbf{S O}(4) \subset \mathbf{G}_{\mathbf{2}}$ then we have the following decomposition of the $\mathbf{G}_{\mathbf{2}}$-representation $\mu$,

$$
\mu=S^{20} \oplus S^{11}
$$

Proof. See the proof of [Yokog, Theorem 1.10.1], but instead of the described map use

$$
\varphi: \mathbf{S p}(1)_{+} \times \mathbf{S p}(1)_{-}: \mathrm{G}_{2}^{\gamma}, \quad\left(\mathrm{m}+\mathrm{a} e_{4}\right)=\mathrm{pm} \overline{\mathrm{p}}+\mathrm{qa} \overline{\mathrm{p}} \mathrm{e}_{4} .
$$

(see the reference for the notation; we swapped $\mathbf{S p}(1)_{+}$with $\mathbf{S p}(1)_{-}$to get $S^{20}$ instead of $S^{02}$ ). Then the representations are $S^{20}$ and $S^{11}$ where the ismorphism of the second is given by $\mathbb{H} \rightarrow \mathbb{H}, x \mapsto \bar{x}$.

Remark 2.39. The identifiaction $x \mapsto \bar{\chi}$ will haunt us later on, but it is necessary beacuse we want to work with anti self-dual instantons. 


\section{The representations of $\operatorname{Spin}(7)$}

The representations of $\operatorname{Spin}(7)$ can again be read off the Coxeter-Dynkin diagram $B_{3}$. For details, see [Halo3; Varo1].

Table 2.3: Some Irreducible Real Representations of Spin(7).

\begin{tabular}{cccl}
\hline Highest Weight & Name & Dimension & Description \\
\hline$(0,0,0)$ & $\mathbb{R}$ & $\mathbb{1}$ & trivial representation \\
$(1,0,0)$ & $\mathbb{R}^{7}$ & 7 & representation from $\operatorname{SO}(7)$ \\
$(0,1,0)$ & $\mathbf{S}_{8}$ & 8 & spin representation \\
$(0,0,1)$ & $\mathfrak{s p i n}(7)$ & 21 & the adjoint representation \\
\hline
\end{tabular}

Figure 2.4: The Coxeter-Dynkin Diagram of $\mathfrak{s o}(7, \mathbb{C})$.

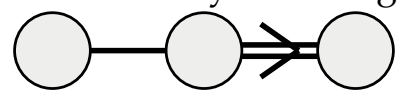

\subsection{Manifolds of Special Holonomy}

\subsubsection{Hyperkähler structure}

Definition 2.40 (Hyperkähler structure). A 4-dimensional oriented Riemannian manifold $M$ is said to have a hyperkähler structure if its holonomy group reduces to $\mathbf{S p}(1) \subset$ SO(4). The structure is called an integrable hyperkähler structure if the Levi-Civita connection reduces too.

Proposition 2.41 (Description by local forms). Let M be a 4-dimensional oriented Riemannian manifold and

$$
\mathcal{F}=\left\{\left(\omega_{1}, \omega_{2}, \omega_{3}\right) \in\left(\Lambda^{2} T_{x}^{\vee} M\right)^{\oplus 3} \mid \exists p \in \operatorname{Fr}_{S O}(M)_{x}: p^{*} \omega_{i}=\omega_{i}^{\prime}\right\}
$$

be the admissible bundle of $\omega_{1}^{\prime}, \omega_{2}^{\prime}$ and $\omega_{3}^{\prime}$. Then a hyperkähler structure is equivalent to a section

$$
\left(\omega_{1}, \omega_{2}, \omega_{3}\right) \in \Gamma(\mathcal{F}),
$$

and it is integrable if and only if the sections are parallel.

Proof. This follows immediately from the holonomy theorem (see e.g. [Bauog, Satz 5.3]) and Proposition 2.28.

Lemma 2.42 (Hitchin). Let $M$ be an oriented Riemannian manifold with hyperkähler structure $\left(\omega_{1}, \omega_{2}, \omega_{3}\right)$. Then the structure is integrable if and only if

$$
\mathrm{d} \omega_{1}=\mathrm{d} \omega_{2}=\mathrm{d} \omega_{3}=0 .
$$


Notation 2.43. In the following $\Lambda_{j}^{i}$ will denote a $j$-dimensional subspace of the $i$-th exterior product of $T^{\vee} M$.

Proposition $2.44\left(\Lambda^{\bullet}\right.$ of a hyperkähler manifold). Let $M$ be a 4-dimensional hyperkähler manifold. Then

\begin{tabular}{ccc}
\hline Space & Decomposition & Isomorphic to by $\star$ \\
\hline$\Lambda^{0} T^{\vee} M$ & $\Lambda_{1}^{0}$ & $\Lambda^{4} T^{\vee} M$ \\
$\Lambda^{1} T^{\vee} M$ & $\Lambda_{4}^{1}$ & $\Lambda^{3} T^{\vee} M$ \\
$\Lambda^{2} T^{\vee} M$ & $3 \Lambda_{1}^{2} \oplus \Lambda_{3}^{2}$ & \\
\hline
\end{tabular}

where $\Lambda_{1}^{\cdot}=\mathbb{R}$ are the trivial representations, $\Lambda_{4}^{1}=\mathbb{C}_{\mathbb{R}}^{2}$ is the realified tautological representation and $\Lambda_{3}^{2}=\mathfrak{s u}(2)$ is the adjoint representation.

Proof. $\Lambda^{1} \mathrm{~T}^{\vee} M=\Lambda_{4}^{1}=\mathbb{C}_{\mathbb{R}}^{2}$ follows immediately by restricting $\mathbf{S O}(4) \frown \mathbb{R}^{4}$ to $\mathbf{S U}(2) \hookrightarrow$ SO(4). Furthermore

$$
\Lambda^{2} T^{\vee} M=\Lambda^{2} \mathbb{R}^{4}=\Lambda_{+}^{2} \oplus \Lambda_{-}^{2}
$$

as $\mathbf{S O}(4)$ representations. It is then a tedious but simple calculation to show that $\Lambda_{+}^{2} \cong 3 \mathbb{R}$ and $\Lambda_{-}^{2} \cong \mathfrak{s u}(2)$ as $\mathbf{S U}(2)$ representations. First note that $\mathbf{S U}(2)$ is simply connected, so that we can equivalently work with the its Lie Algebra $\mathfrak{s u}(2)$.

We use the usual orthogonal basis of $\Lambda_{ \pm}^{2}$

$$
f_{ \pm}^{1}=e^{12} \pm e^{34}, \quad f_{ \pm}^{2}=e^{13} \mp e^{24}, \quad f_{ \pm}^{3}=e^{14} \pm e^{23} .
$$

Here $e^{i j}=e^{i} \wedge e^{j}$ and $e^{1}, \ldots, e^{4}$ is the dual of the standard basis $e_{1}, \ldots, e_{4}$ of $\mathbb{R}^{4}$. If we identify $\mathbb{C}_{\mathbb{R}}^{2}$ with $\mathbb{R}^{4}$, we have the identification

$$
e_{1} \leftrightarrow\left(\begin{array}{l}
1 \\
0
\end{array}\right), \quad e_{2} \leftrightarrow\left(\begin{array}{l}
i \\
0
\end{array}\right), \quad e_{3} \leftrightarrow\left(\begin{array}{l}
0 \\
1
\end{array}\right), \quad e_{4} \leftrightarrow\left(\begin{array}{l}
0 \\
i
\end{array}\right) .
$$

Using the standard basis of $\mathfrak{s u}(2)$ given by

$$
u_{1}=\left(\begin{array}{cc}
i & 0 \\
0 & -i
\end{array}\right), \quad u_{2}=\left(\begin{array}{cc}
0 & 1 \\
-1 & 0
\end{array}\right), \quad u_{3}=\left(\begin{array}{ll}
0 & i \\
i & 0
\end{array}\right),
$$

the representation $\rho$ of $\mathfrak{s u}(2)$ on $\mathbb{R}^{4} \cong \mathbb{C}_{\mathbb{R}}^{2}$ is given by left multiplication of the above matrices. A quick inspection shows that the dual representation $X \mapsto-\rho(X)^{\top}$ has exactly the same matrices (with respect to the dual basis) and hence the induced representation $\Lambda^{2} \rho^{\vee}$ acts by

\begin{tabular}{c|ccc}
$\cdot$ & $f_{+}^{1}$ & $f_{+}^{2}$ & $f_{+}^{3}$ \\
\hline$u_{1}$ & 0 & 0 & 0 \\
$u_{2}$ & 0 & 0 & 0 \\
$u_{3}$ & 0 & 0 & 0
\end{tabular}

\begin{tabular}{c|ccc}
$\cdot$ & $\mathrm{f}_{-}^{1}$ & $\mathrm{f}_{-}^{2}$ & $\mathrm{f}_{-}^{3}$ \\
\hline $\mathrm{u}_{1}$ & 0 & $-2 \mathrm{f}_{-}^{3}$ & $2 \mathrm{f}_{-}^{2}$ \\
$\mathrm{u}_{2}$ & $-2 \mathrm{f}_{-}^{3}$ & 0 & $2 \mathrm{f}_{-}^{1}$ \\
$\mathrm{u}_{3}$ & $-2 \mathrm{f}_{-}^{2}$ & $2 \mathrm{f}_{-}^{1}$ & 0
\end{tabular}


which shows that the representation on $\Lambda_{+}^{2}$ is trivial and the isomorphism induced by $f_{-}^{1} \mapsto u_{1}, f_{-}^{2} \mapsto-u_{2}$ and $f_{-}^{3} \mapsto u_{3}$ gives an $\mathfrak{s u}(2)$-module isomorphisms between $\mathfrak{s u}(2)$ and $\Lambda_{-}^{2}$.

Proposition 2.45 (Description of the various representations). We have the following description of the various representations of proposition 2.44)

$$
\begin{aligned}
\Lambda_{3}^{2} & =\bigcap_{i=1}^{3} \operatorname{ker}\left(\mathrm{F}\left(\omega_{i}\right)\right) \\
3 \Lambda_{1}^{2} & =\left\langle\omega_{1}\right\rangle \oplus\left\langle\omega_{2}\right\rangle \oplus\left\langle\omega_{3}\right\rangle
\end{aligned}
$$

Proof. The second assertion follows immediately from the proof of proposition (2.44). To see the first, note that wedging with $\omega_{i}$ gives a $\mathbf{S U}(2)$-map $3 \Lambda_{1}^{2} \oplus \Lambda_{3}^{2} \rightarrow \Lambda_{1}^{4}$, and a quick check shows that $\omega_{i} \wedge \omega_{i} \neq 0$, so the kernel of the map is of dimension 5. This shows the first assertion.

\subsubsection{Hypo structure}

Definition 2.46 (Hypo structure). A 5-dimensional oriented Riemannian manifold $M$ possesses a hypo structure, if the structure group of the frame bundle reduces to $\mathrm{l}_{2}: \mathbf{S U}(2) \hookrightarrow \mathbf{S O}(5)$. If, in addition, the Levi-Civita connection reduces, we say that $M$ possesses an integrable hypo structure.

Proposition 2.47 (Descritpion by local forms). Let $\mathrm{M}$ be a 5-dimensional oriented Riemannian manifold and let

$$
\mathcal{F}_{x}=\left\{\left(\eta, \eta_{1}, \eta_{2}, \eta_{3}\right) \in\left(\Lambda^{2} T_{x}^{\vee} M\right)^{\oplus 3} \oplus T_{x}^{\vee} M \mid \exists p \in \operatorname{Fr}_{S O}(M): \begin{array}{l}
p^{*} \eta=\eta^{\prime}, \\
p^{*} \eta_{i}=\eta_{i}^{\prime}
\end{array}\right\}
$$

be the admissible subbundle to the hypo structure forms. Then a hypo structure on $\mathrm{M}$ is given by a sections

$$
\left(\eta, \omega_{1}, \omega_{2}, \omega_{3}\right) \in \Gamma(\mathcal{F})
$$

and it is integrable if and only if all sections are parallel.

Proof. This follows from the holonomy theorem (see e.g. [Bauog, Satz 5.3]) and Proposition 2.28 ,

Proposition $2.48\left(\Lambda^{\bullet}\right.$ of a hypo manifold). Let $M$ be a manifold with a hypo structure. Then the exterior powers of the cotangent bundle decompose as follows

\begin{tabular}{ccc}
\hline Space & Decomposition & Isomorphic to by $\star$ \\
\hline$\Lambda^{0} T^{\vee} M$ & $\Lambda_{1}^{0}$ & $\Lambda^{5} T^{\vee} M$ \\
$\Lambda^{1} T^{\vee} M$ & $\Lambda_{1}^{1} \oplus \Lambda_{4}^{1}$ & $\Lambda^{4} T^{\vee} M$ \\
$\Lambda^{2} T^{\vee} M$ & $\Lambda_{4}^{2} \oplus 3 \Lambda_{1}^{2} \oplus \Lambda_{-}^{2}$ & $\Lambda^{3} T^{\vee} M$ \\
\hline
\end{tabular}


where $\Lambda_{1}=\mathbb{R}$ are the trivial representations, $\Lambda_{4}=\mathbb{C}_{\mathbb{R}}^{2}$ are the realified tautological representations and $\Lambda_{-}^{2}=\mathfrak{s u}(2)$ is the adjoint representation.

Proposition 2.49 (Description of the various representations). We have the following description of the representations of proposition 2.48

$$
\begin{aligned}
\Lambda_{1}^{1} & =\langle\eta\rangle \\
\Lambda_{4}^{1} & =\operatorname{ker}\left(\left.\iota_{\eta^{\sharp}}\right|_{\Lambda^{1}}\right) \\
\Lambda_{4}^{2} & =\operatorname{im}\left(\left.\mathrm{F}(\eta)\right|_{\Lambda^{1}}\right) \\
3 \Lambda_{1}^{2} & =\left\langle\omega_{1}\right\rangle \sqcup\left\langle\omega_{2}\right\rangle \sqcup\left\langle\omega_{3}\right\rangle \\
\Lambda_{-}^{2} & =\operatorname{ker}\left(\left.F\left(\eta_{2}\right)\right|_{\Lambda^{2}}\right) \cap \operatorname{ker}\left(\left.F\left(\eta_{2}\right)\right|_{\Lambda^{2}}\right) \cap \operatorname{ker}\left(\left.F\left(\eta_{3}\right)\right|_{\Lambda^{2}}\right) \cap \operatorname{ker}\left(\left.F(\eta)\right|_{\Lambda^{2}}\right)
\end{aligned}
$$

Proof. This is can be checked by calculating with the hypo structure forms on $\mathbb{R}^{5}$ and realizing that all maps above are $\mathbf{S U}(2)$-equivariant.

\subsubsection{SU(3) structure}

Definition 2.50. A 6-dimensional oriented Riemannian manifold $M$ possesses a SU(3)structure if the structure group of the frame bundle reduces to $\iota_{3}: \mathbf{S U}(3) \hookrightarrow \mathbf{S O}(6)$. If, in addition, the Levi-Civita connection reduces, we say that $M$ possesses an integrable SU(3)-structure.

Proposition 2.51. Let $M$ be a 6-dimensional oriented Riemannian manifold and let

$$
\mathcal{F}_{x}=\left\{(\omega, \Omega) \in \Lambda^{2} \mathrm{~T}_{x}^{\vee} M \oplus \Lambda^{3} \mathrm{~T}_{x}^{\vee} M \mid \exists p \in \operatorname{Fr}_{\mathbf{S O}}(M): \begin{array}{l}
p^{*} \omega=\omega^{\prime}, \\
p^{*} \Omega=\Omega^{\prime}
\end{array}\right\}
$$

be the corresponding admissible bundle. A SU(3) structure on $\mathrm{M}$ is a pair of sections

$$
(\omega, \Omega) \in \Gamma(\mathcal{F}),
$$

and it is integrable if and only if the sections are parallel.

Proof. This follows from the holonomy theorem (see e.g. [Bauog, Satz 5.3]) and Proposition 2.28 ,

Proposition $2.52\left(\Lambda^{\bullet}\right.$ of a SU(3) manifold). Let $M$ be a 6-dimensional SU(3)-manifold. Then

\begin{tabular}{ccc}
\hline Space & Decomposition & Isomorphic to by $\star$ \\
\hline$\Lambda^{0} T^{\vee} M$ & $\Lambda_{1}^{0}$ & $\Lambda^{6} T^{\vee} M$ \\
$\Lambda^{1} T^{\vee} M$ & $\Lambda_{6}^{1}$ & $\Lambda^{5} T^{\vee} M$ \\
$\Lambda^{2} T^{\vee} M$ & $\Lambda_{1}^{2} \oplus \Lambda_{6}^{2} \oplus \Lambda_{8}^{2}$ & $\Lambda^{4} T^{\vee} M$ \\
$\Lambda^{3} T^{\vee} M$ & $2 \Lambda_{1}^{3} \oplus \Lambda_{6}^{3} \oplus \Lambda_{12}^{3}$ & \\
\hline
\end{tabular}


where the representations of the decomposition are uniquely determined by their dimensions (compare to Table 2.1).

Proof. See e.g. [Xuo8, section 2.1.1].

\subsection{4 $\mathrm{G}_{2}$ structure}

Definition 2.53. A 7-dimensional oriented Riemannian manifold $M$ possesses a $\mathbf{G}_{\mathbf{2}}-$ structure if the structure group of the frame bundle reduced to $\iota_{4}: \mathbf{G}_{2} \hookrightarrow \mathbf{S O}(7)$. If, in addition, the Levi-Civita connection reduces, we say that $M$ possesses an integrable $\mathbf{G}_{\mathbf{2}}$-structure.

Proposition 2.54. Let $M$ be a 7-dimensional oriented Riemannian manifold and let

$$
\mathcal{F}_{x}=\left\{\varphi \in \Lambda^{3} T_{x}^{\vee} M \mid \exists p \in \operatorname{Fr}_{\mathbf{S O}}(M): p^{*} \varphi=\varphi^{\prime}\right\}
$$

be the corresponding admissible bundle. A $\mathbf{G}_{\mathbf{2}}$ structure on $\mathrm{M}$ is section

$$
\varphi \in \Gamma(\mathcal{F}),
$$

and it is integrable if and only if the section is parallel.

Proof. This follows from the holonomy theorem (see e.g. [Bauog, Satz 5.3]) and Proposition 2.28 .

Lemma 2.55. A section $\varphi \in \Gamma(\mathcal{F})$ is parallel if and only if $\varphi$ is closed and co-closed.

Proposition $2.56\left(\Lambda^{\bullet}\right.$ of a $\mathbf{G}_{\mathbf{2}}$ manifold). Let $M$ be a 7-dimensional $\mathbf{G}_{\mathbf{2}}$-manifold. Then

\begin{tabular}{ccc}
\hline Space & Decomposition & Isomorphic to by $\star$ \\
\hline$\Lambda^{0} T^{\vee} M$ & $\Lambda_{1}^{0}$ & $\Lambda^{7} T^{\vee} M$ \\
$\Lambda^{1} T^{\vee} M$ & $\Lambda_{7}^{1}$ & $\Lambda^{6} T^{\vee} M$ \\
$\Lambda^{2} T^{\vee} M$ & $\Lambda_{7}^{2} \oplus \Lambda_{14}^{2}$ & $\Lambda^{5} T^{\vee} M$ \\
$\Lambda^{3} T^{\vee} M$ & $\Lambda_{1}^{3} \oplus \Lambda_{7}^{3} \oplus \Lambda_{27}^{3}$ & $\Lambda^{4} T^{\vee} M$ \\
\hline
\end{tabular}

where the representations of the decomposition are uniquely determined by their dimensions (compare to Table 2.2.).

Proof. See e.g. [Bry87]. 
Proposition 2.57. Let $\mathrm{M}$ be a 7-dimensional $\mathbf{G}_{\mathbf{2}}$-manifold. Then

$$
\begin{aligned}
\Lambda_{7}^{2} & =\operatorname{im}\left(\left.\mathrm{G}(\psi)\right|_{\Lambda^{1}}\right)=\operatorname{Eig}\left(\left.\mathrm{G}(\varphi)\right|_{\Lambda^{2}} ; 2\right) \\
\Lambda_{14}^{2} & =\operatorname{ker}\left(\left.\mathrm{F}(\psi)\right|_{\Lambda^{2}}\right)=\operatorname{Eig}\left(\left.\mathrm{G}(\varphi)\right|_{\Lambda^{2}} ;-1\right) \\
\Lambda_{1}^{3} & =\langle\varphi\rangle \\
\Lambda_{7}^{3} & =\operatorname{im}\left(\left.\mathrm{G}(\varphi)\right|_{\Lambda^{1}}\right) \\
\Lambda_{27}^{3} & =\operatorname{ker}\left(\left.\mathrm{F}(\varphi)\right|_{\Lambda^{3}}\right) \cap \operatorname{ker}\left(\left.\mathrm{F}(\psi)\right|_{\Lambda^{3}}\right)
\end{aligned}
$$

where the representations of the decomposition are uniquely determined by their dimensions (compare to Table 2.2).

Proposition 2.58. There is a $\mathbf{G}_{\mathbf{2}}$-structure on $\Lambda_{+}^{2} \mathrm{X}$ of a 4-dimensional Riemannian manfiold. If $\mathrm{X}$ is an anti self-dual Einstein manifold, then an integrable $\mathbf{G}_{\mathbf{2}}$-structure can be defined on $\Lambda_{+}^{2} \mathrm{X}$. These structures reduce algebraically to $\mathbf{S O}(4)$.

Proof. See [Sal89, Theorem 11.10].

\subsection{5 $\operatorname{Spin}(7)$ structure}

Definition 2.59. An 8-dimensional oriented Riemannian manifold $M$ is said to posses a $\operatorname{Spin}(7)$-structure if the structure group of the frame bundle reduced to $\iota_{5}: \operatorname{Spin}(7) \hookrightarrow$ SO $(8)$. If, in addition, the Levi-Civita connection reduces, we say that $M$ possesses an integrable Spin(7)-structure.

Proposition 2.60. Let $\mathrm{M}$ be a 8-dimensional oriented Riemannian manifold and let

$$
\mathcal{F}_{x}=\left\{\Theta \in \Lambda^{4} \mathrm{~T}_{x}^{\vee} M \mid \exists p \in \operatorname{Fr}_{\mathbf{S O}}(M): p^{*} \Theta=\Theta^{\prime}\right\}
$$

be the corresponding admissible bundle. A Spin(7) structure on $\mathrm{M}$ is section

$$
\Theta \in \Gamma(\mathcal{F}),
$$

and it is integrable if and only if the section is parallel.

Proof. This follows from the holonomy theorem (see e.g. [Bauog, Satz 5.3]) and Proposition 2.28 ,

Lemma 2.61. A section $\Theta \in \Gamma(\mathcal{F})$ is parallel if and only if $\Theta$ is closed.

Proposition $2.62\left(\Lambda^{\bullet}\right.$ of a Spin(7) manifold). Let $M$ be a 7-dimensional Spin(7)-manifold. Then

\begin{tabular}{ccc}
\hline Space & Decomposition & Isomorphic to by $\star$ \\
\hline$\Lambda^{0} \mathrm{~T}^{\vee} M$ & $\Lambda_{1}^{0}$ & $\Lambda^{8} \mathrm{~T}^{\vee} M$ \\
$\Lambda^{1} \mathrm{~T}^{\vee} M$ & $\Lambda_{8}^{1}$ & $\Lambda^{7} \mathrm{~T}^{\vee} M$ \\
$\Lambda^{2} \mathrm{~T}^{\vee} M$ & $\Lambda_{7}^{2} \oplus \Lambda_{21}^{2}$ & $\Lambda^{6} \mathrm{~T}^{\vee} M$ \\
$\Lambda^{3} \mathrm{~T}^{\vee} M$ & $\Lambda_{8}^{3} \oplus \Lambda_{48}^{3}$ & $\Lambda^{5} \mathrm{~T}^{\vee} M$ \\
$\Lambda^{4} \mathrm{~T}^{\vee} M$ & $\Lambda_{1}^{4} \oplus \Lambda_{7}^{4} \oplus \Lambda_{27}^{4} \oplus \Lambda_{35}^{4}$ & - \\
\hline
\end{tabular}


Proof. See e.g. [Bry87].

Proposition 2.63. Let $\mathrm{M}$ be a 8-dimensional Spin(7)-manifold. Then

$$
\begin{aligned}
\Lambda_{7}^{2} & =\operatorname{Eig}\left(\left.G(\Theta)\right|_{\Lambda^{2}} ; 3\right) \\
\Lambda_{21}^{2} & =\operatorname{Eig}\left(\left.G(\Theta)\right|_{\Lambda^{2}} ;-1\right) \\
\Lambda_{8}^{3} & =\operatorname{im}\left(\left.G(\Theta)\right|_{\Lambda^{1}}\right) \\
\Lambda_{48}^{3} & =\operatorname{ker}\left(\left.\mathrm{F}(\Theta)\right|_{\Lambda^{3}}\right) \\
\Lambda_{1}^{4} & =\langle\Theta\rangle \\
\Lambda_{35}^{4} & =\operatorname{Eig}\left(\star_{8}||_{\Lambda^{4}} ;-1\right)
\end{aligned}
$$

Proof. See $[\overline{B r y} 87]$ for a justification and a description of the remaining spaces (which we are not interested in here).

\subsubsection{Connection of Holonomies}

Corollary 2.64. If we apply the above to the holonomy groups of manifolds, we get the following facts.

- An 8-manifold with (integrable) Spin(7) holonomy and a global parallel non-vanishing vector field has (integrable) holonomy in $\mathbf{G}_{\mathbf{2}}$.

- A 7-manifold with (integrable) $\mathbf{G}_{\mathbf{2}}$ holonomy and a global parallel non-vanishing vector field has (integrable) holonomy in $\mathbf{S U}(3)$.

- A 6-manifold with (integrable) SU(3) holonomy and a global parallel non-vanishing vector field has (integrable) holonomy in $\mathbf{S U}(2)$.

Proof. Apply the holonomy principle and use Corollary 2.29.

Remark 2.65. We can also formulate this the following way. If $\mathbb{R} \times M$ is a $\operatorname{Spin}(7)$ manifold, then $M$ is a $\mathbf{G}_{\mathbf{2}}$ manifold. If $\mathbb{R} \times M$ is a $\mathbf{G}_{\mathbf{2}}$-manifold, then $M$ is a $\mathbf{S U}(3)$ manifold. If $\mathbb{R} \times M$ is a $\mathbf{S U}(3)$ manifold, then $M$ is a manifold with hypo structure. Finally if $\mathbb{R} \times M$ is a manifold with hypo structure, then $M$ is a $\mathbf{S U}(2)$ manifold.

Remark 2.66. We will be interested in an additional case. Assume we have manifold $M$ with (integrable) Spin(7)-structure, which has an free and proper action by $\mathbf{S O}(3)$, such that the quotient is flat (in the sense of the induced submersion by the Riemannian submersion). This means that the quotient is locally a Riemannian product and by the decomposition theorem of de Rham and $\mathrm{Wu}$, this means the distribution induced by the action of $\mathbf{S O}(3)$ in $\mathrm{TM}$ is invariant under the action of the holonomy group. Via parallel transport we can restrict the discussion to a single tangent space.

As discussed in Lemma 2.38 and thereafter, the decomposition of $\mathrm{T}_{\mathrm{p}} \mathrm{M}$ for holonomy groups

$$
\mathrm{SU}(2) \subset \mathrm{G}_{2} \subset \operatorname{Spin}(7)
$$


decomposes into dimensions $4+1+1+1+1$, whereas the decomposition for

$$
\operatorname{SO}(4) \subset \mathbf{G}_{2} \subset \operatorname{Spin}(7)
$$

is into dimensions $4+3+1$.

This shows that a $\mathbf{S O}(3)$ action cannot achieve the former, and it can only achieve the latter if we act on the "the three extra dimensions of $\mathbf{G}_{\mathbf{2}}$ over $\mathbf{S O}(4)$ " (otherwise this would imply the existence of a parallel vector field on $\mathbf{S O}(3)$ ). 


\section{Chapter 3}

\section{Hyperkähler Reduction}

\subsection{Introduction}

The Hyperkähler Reduction is a cousin of the Symplectic Reduction applicable to the setting where the starting manifold $M$ is hyperkähler and the involved data, the action of an auxiliary group $\mathrm{G}$ and the moment map $\mu$, respect this structure. It is well known, that this implies that the final manifold, the quotient of a preimage of a central regular value of $\mu$ by G, also is a hyperkähler manifold. This however is not all that is special about the hyperkähler reduction.

In their paper [GN92] T. Gocho and H. Nakajima find some interesting relations between various geometrical quantities involved in this construction. The paper uses calculations in the tangent bundle to show these relations.

We will present a different approach in this work by lifting the calculation onto the involved principal bundles. Although quite a bit longer than the original work, it highlights the role the quaternionic structure plays in the construction. The length can be partly attributed to the need to introduce basic notions in this setting, e.g. the section 3.4.5 Riemannian Submersions which recovers the fundamentals of O'Neill's theory in the principal bundle setting.

The aim of this chapter is to show that these relations can be derived fundamentally from the structure of quaternionic matrices, when embedded into real matrices. It does so, by first deriving equation (3.68), which does not need the involved quaternionic structures. Then this equation is compared to the quaternionic world (3.69), and this comparison yields all the relations that we long for. It then just remains to decipher the implied relations for the quaternionic components.

The section 3.2 Definitions recalls the basic notions involved in hyperkähler geometry and in particular in a hyperkähler reduction. Of utmost importance to the next sections are the notions of reduction and extension of principal bundles. Further it describes a recipe to compare forms on the manifolds and the involved principal bundles. 
Section 3.3 Setting first discusses the tangent bundle of $M$ and how its quaternionic structure behaves with respect to the reduction. This structure allows for various reductions of the principal bundle of frames of $M$. These bundles lie at the heart of the construction in this work.

The following section inspects the involved forms with respect to the bundles discussed. Concretely we will trace the reductions of the Levi-Civita connection and tautological form starting from the principal bundle of frames of $M$ all the way to the principal bundle of frames of the quotient N. A quick excursion is made in this section, explaining the fundamentals of Riemannian Submersions in the principal bundle language.

The last section 3.5 Final Results uses the preceding work to recover the results of Gocho and Nakajima, and show a small novelty. It is this section where the relation between the quaternionic structure and the results is investigated.

\subsection{Definitions}

Let us define some standard notions. Throughout this chapter, let $M$ be a smooth oriented Riemannian manifold of dimension $4 \mathrm{~m} \in \mathbb{N}$, and $\mathrm{G}$ a smooth Lie group of dimension $k \in \mathbb{N}$.

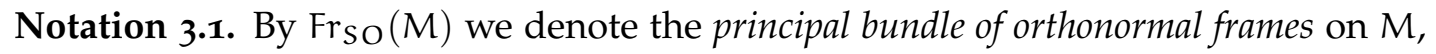

$$
\operatorname{Fr}_{S O}(M)=\left\{p: \mathbb{R}^{4 m} \rightarrow T_{x} M: p \text { is an oriented orthogonal isomorphism }\right\} .
$$

Notation 3.2. By $\theta^{M} \in \Omega^{1}\left(\operatorname{Fr}_{S O}(M), \mathbb{R}^{4 m}\right)$ we denote the soldering form of $\operatorname{Fr}_{S O}(M)$

$$
\theta_{\mathrm{p}}^{M}(\xi)=p^{-1} \circ \mathrm{D} \pi_{\mathrm{p}}(\xi), \quad p \in \mathrm{Fr}_{\mathrm{SO}}(M), \xi \in \mathrm{T}_{\mathrm{p}} \operatorname{Fr}_{\mathrm{SO}}(\mathrm{M}),
$$

where $\pi: \mathrm{Fr}_{\mathrm{SO}}(\mathrm{M}) \rightarrow \mathrm{M}$ is the projection.

Let $\varphi \in \Omega^{1}\left(\operatorname{Fr}_{\mathrm{SO}}(M), \mathfrak{s o}(4 \mathrm{~m})\right)^{\mathbf{S O}(4 \mathrm{~m})}$ denote the Levi-Civita connection of $(M, g)$. Then $\varphi$ satisfies

- $\mathrm{R}_{\mathrm{g}}^{*} \varphi=\operatorname{Ad}_{\mathrm{g}^{-1}} \circ \varphi$, for all $\mathrm{g} \in \mathbf{S O}(4 \mathrm{~m})$,

- $\varphi\left(\mathrm{K}^{\xi}\right)=\xi$ for all $\xi \in \mathfrak{s o}(4 \mathrm{~m})$, where $\mathrm{K}^{\xi}$ is the fundamental vector field to the lie algebra element $\xi$, i.e.

$$
K_{p}^{\xi}=\left.\frac{d}{d t}\right|_{t=0}(p \exp (t \xi)),
$$

- $\mathrm{d} \theta+\varphi \wedge \theta=0$, i.e. $\varphi$ has zero torsion.

Definition 3.3 (Hyperkähler Manifold). A Riemannian manifold $(M, g)$ with a triple of almost complex structures I, J, K,

$$
\mathrm{I}, \mathrm{J}, \mathrm{K}: \mathrm{TM} \rightarrow \mathrm{TM}, \quad \mathrm{I}^{2}=\mathrm{J}^{2}=\mathrm{K}^{2}=-\mathrm{id}_{\mathrm{TM}},
$$


which satisfy the quaternionic relation $\mathrm{IJ}=\mathrm{K}$ and are compatible with the metric,

$$
g(-,-)=g(I-, I-)=g(J-, J-)=g(K-, K-),
$$

is called a hyperkähler manifold (hk-manifold) if the two-forms corresponding to I, J and $\mathrm{K}$ are closed, i.e.

$$
\mathrm{d} \omega_{\mathrm{A}}=0, \quad \omega_{\mathrm{A}}(-,-)=\mathrm{g}(\mathrm{A}-,-), \quad A \in\{\mathrm{I}, \mathrm{J}, \mathrm{K}\} .
$$

Proposition 3.4 (Alternative Characterization). $\left(\mathrm{M}^{4 \mathrm{~m}}, \mathrm{~g}\right)$ is a hyperkähler manifold if and only if the structure group of $\mathrm{Fr}_{\mathrm{SO}}(\mathrm{M})$ reduces to $\mathbf{S p}(\mathrm{m})$ and the Levi-Civita connection on $\operatorname{Fr}_{\mathrm{SO}}(\mathrm{M})$ reduces to a connection on

$$
\operatorname{Fr}_{\mathrm{Sp}}(\mathrm{M})=\left\{\mathrm{p}: \mathbb{H}^{\mathrm{m}} \rightarrow \mathrm{T}_{\mathrm{x}} \mathrm{M}: \mathrm{p} \text { is a } \mathbb{H} \text {-linear isomorphism }\right\},
$$

i.e. the horizontal subspaces are tangent to the submanifold $\operatorname{Fr}_{\mathrm{Sp}}(M) \subset \operatorname{Fr}_{\mathrm{SO}}(M)$.

Note that in the dual formulation the condition on the horizontal subspaces is that $\varphi$ reduces to a connection on $\operatorname{Fr}_{S p}(M)$. Precisely this means that $j^{*} \varphi$ is a connection on $\operatorname{Fr}_{S p}(M)$, where $j: \operatorname{Fr}_{S p}(M) \rightarrow \operatorname{Fr}_{S O}(M)$.

Definition 3.5 (Hyperkähler Action). We say a group G acts hyperkähler on a hyperkähler manifold $(M, g, I, J, K)$, if $G$ acts on $M$ and this action preserves the metric $g$ and the hyperkähler structures I, J and K, i.e.

$$
R_{h}^{*} \omega_{A}=\omega_{A} \quad \forall A \in\{I, J, K\}, \quad R_{h}^{*} g=g,
$$

for all $h \in G$. (In this case we used a right action of $G$ on $M$, but this definition does not require so).

Definition 3.6 (tri-hamiltonian action). A hyperkähler action of $G$ on $M$ is called a tri-hamiltonian action, if G-equivariant moment maps

$$
\mu_{\mathrm{I}}, \mu_{\mathrm{J}}, \mu_{\mathrm{K}}: M \rightarrow \mathfrak{g}^{*}
$$

exist, i.e.

$$
\begin{aligned}
& \mu_{\mathrm{A}}(\mathrm{x} . \mathrm{h})=\mathrm{Ad}_{\mathrm{h}^{-1}}^{*} \circ \mu_{\mathrm{A}}(\mathrm{x}) \quad \forall \mathrm{x} \in \mathrm{M}, \quad \forall \mathrm{h} \in \mathrm{G}, \quad \forall \mathrm{A} \in\{\mathrm{I}, \mathrm{J}, \mathrm{K}\}, \\
& \left\langle\xi, \mathrm{d} \mu_{\mathcal{A}}(\eta)\right\rangle=\omega_{\mathcal{A}}\left(\mathrm{K}^{\xi}, \eta\right) \quad \forall \eta \in T M, \quad \forall \xi \in \mathfrak{g}, \quad \forall A \in\{\mathrm{I}, \mathrm{J}, \mathrm{K}\} .
\end{aligned}
$$

The moment maps of a tri-hamiltonian action are also often considered together as a map $\mu=\left(\mu_{\mathrm{I}}, \mu_{\mathrm{J}}, \mu_{\mathrm{K}}\right): M \rightarrow \mathbb{R}^{3} \otimes \mathfrak{g}^{*}$. 


\subsubsection{Reduction and Extensions}

Let $\pi: P \rightarrow M$ be a principal bundle with structure group $G$. A reduction of $P$ is a principal bundle $\mathrm{Q} \rightarrow \mathrm{M}$ with structure group $\mathrm{H}$ and maps

$$
\lambda: H \rightarrow G, \quad f: Q \rightarrow P,
$$

a Lie homomorphism and a smooth map respectively, such that the following diagram commutes.

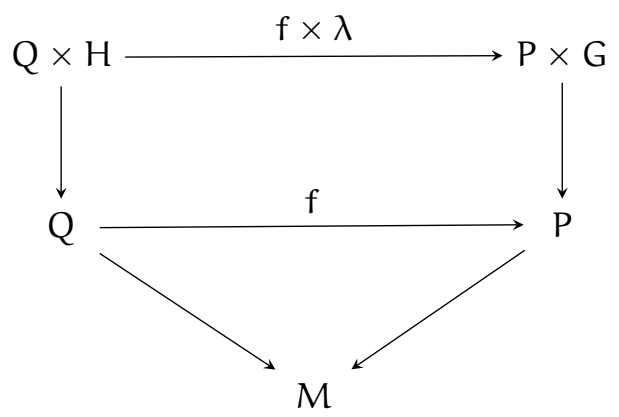

The vertical maps above are the group actions on the principal bundles. An extension of $P$ is a principal bundle $\tilde{Q} \rightarrow M$ of structure group $\tilde{H}$ with maps $\tilde{\lambda}: G \rightarrow \tilde{H}$ and $\tilde{f}: P \rightarrow \tilde{Q}$, such that $P$ is a reduction of $\tilde{Q}$.

Given a connection $\phi^{\mathrm{P}}$ on $\mathrm{P}$, then there is a unique connection $\phi^{\tilde{Q}}$ on $\tilde{\mathrm{Q}}$ such that

$$
\tilde{f}^{*} \phi^{\tilde{Q}}=\tilde{\lambda}_{*} \circ \phi^{\mathrm{P}},
$$

where $\tilde{\lambda}_{*}$ is the derivative of $\tilde{\lambda}$ (see e.g. [Bauog, Satz 4.1]). In this sense, a connection is always extendable. If two connections satisfy the equation above, we say that $\phi^{\mathrm{P}}$ extends to $\phi^{\tilde{Q}}$ and $\phi^{\tilde{Q}}$ reduces to $\phi^{\mathrm{P}}$.

On $\mathrm{Q}$ the situation is somewhat more complicated. We will only discuss the situation for the simplest case where $f=i$ and $\lambda$ are the inclusions.

Proposition 3.7 (Reduction of a connection). If $\mathfrak{g}=\mathfrak{h} \oplus \mathfrak{f}$ as H-representations, i.e. $\mathfrak{f} \subset \mathfrak{g}$ is a vector space complement of $\mathfrak{h} \subset \mathfrak{g}$, with the property that

$$
\operatorname{Ad}_{\mathrm{H}}(\mathfrak{f}) \subset \mathfrak{f},
$$

then $\mathrm{pr}_{\mathfrak{h}} \mathrm{i}^{*} \phi^{\mathrm{P}}$ is a connection on $\mathrm{Q}$, where the projection is with respect to the decomposition given above.

Proof. The only thing to note is, that the condition $\operatorname{Ad}_{H}(\mathfrak{f}) \subset \mathfrak{f}\left(\right.$ together with $\operatorname{Ad}_{H}(\mathfrak{h}) \subset$ $\mathfrak{h}$ ) implies that $\mathrm{pr}_{\mathfrak{h}}$ commutes with $\mathrm{Ad}_{\mathfrak{h}}$ for all $h \in H$. The necessary conditions are then easily checked. 
Definition 3.8. We say that $\varphi$ reduces to $Q$ when the horizontal subspaces are tangent to the subbundle $\mathrm{Q} \subset \mathrm{P}$. In the dual formulation this is true if and only if the pulled back connection takes values in the Lie algebra $\mathfrak{h}$, so that no projection is necessary.

Note that a projected connection as in the lemma above can be extended back to $P$. This will however yield a different connection if the original one was not reducible. This also implies that there are in general multiple connections on $\mathrm{P}$ that project onto a given connection on $\mathrm{Q}$.

Remark 3.9. Let $\mathrm{\imath}: \mathrm{Q} \rightarrow \mathrm{Fr}_{\mathrm{SO}}(\mathrm{M})$ denote a reduction of the frame bundle. We call the pull back $\theta^{\mathrm{Q}}$ of $\theta^{\mathrm{M}}$ to $\mathrm{Q}$ again soldering form of $\mathrm{Q}$. Since the diagram

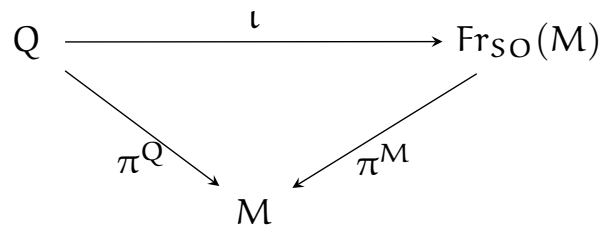

commutes, we have that for all $p \in Q$ and $\xi \in T_{p} Q$

$$
\begin{aligned}
& \theta_{\mathrm{p}}^{\mathrm{Q}}(\xi)=\left(\mathfrak{\imath}^{*} \theta^{\mathrm{M}}\right)_{\mathfrak{p}}(\xi)=\theta_{\mathfrak{\imath}(\mathfrak{p})}^{\mathrm{M}}(\xi)=\mathfrak{\imath}(\mathrm{p})^{-1} \circ\left(\mathrm{D} \pi^{\mathrm{M}}\right)_{\mathfrak{l}(\mathfrak{p})} \circ \mathrm{D} \mathfrak{l}_{\mathfrak{p}}(\xi) \\
& =\mathfrak{l}(p)^{-1} \circ\left(D\left(\pi^{M} \circ \mathfrak{l}\right)\right)_{p}(\xi)=\mathfrak{l}(p)^{-1} \circ\left(D \pi^{Q}\right)_{p}(\xi),
\end{aligned}
$$

so that $\theta_{p}^{Q}=\mathfrak{l}(p)^{-1} \circ D \pi_{p}^{Q}$. In this sense the construction is natural.

\subsubsection{The Correspondence of Forms}

Having a principal bundle of frames $\mathrm{Fr}_{\mathrm{Gl}}(M)$ (or any reduction of it) over a manifold $M$ induces a correspondence between certain forms on the base manifold and the bundle. We will use this correspondence to compare our approach and the one taken in [GN92].

Lemma 3.10 (Correspondence of forms). There is a one-to-one correspondence between horizontal, equivariant and $\mathfrak{g l}(4 \mathrm{~m})$-valued one-forms on the principal bundle of frames, and (global) sections of the vector bundle $\mathrm{T}^{*} \mathrm{M} \otimes \operatorname{End}(\mathrm{TM})$.

Remark 3.11. Note that this is a special case of the correspondence between representation valued forms on a principal bundle and forms with values in associated vector bundles on the base. In the presence of the soldering form, we can give a simple explicit description.

Proof. Let $\omega$ be a horizontal and equivariant one-form on the principal bundle. We induce the wanted section as follows. If $x \in M$ and $\xi, \eta \in T_{x} M$, let $p \in \operatorname{Fr}_{G l}(M)$ be any frame in the fiber of $\pi$ over $x$. Define

$$
s(\omega)(\xi, \eta)=p \omega(\bar{\xi}) \theta(\bar{\eta}),
$$


where $\theta$ is the solder form of $\operatorname{Fr}_{\mathrm{Gl}}(M)$ and $\bar{\xi}$ and $\bar{\eta}$ are lifts of $\xi$ and $\eta$ to $p \in \operatorname{Fr}_{\mathrm{Gl}}(\mathrm{M})$, i.e. $\mathrm{D} \pi(\bar{\xi})=\xi$ and $\mathrm{D} \pi(\bar{\eta})=\eta$. This is well defined, because for a different choice of lifts $\tilde{\xi}$ and $\tilde{\eta}$, the differences $\Delta \xi=\tilde{\xi}-\bar{\xi}$ and $\Delta \eta=\tilde{\eta}-\bar{\eta}$ are vertical, but $\omega$ and $\theta$ are both horizontal forms. A different choice of frame $q=p . g \in \operatorname{Fr}_{G l}(M)$, leads to the calculation

$$
\begin{aligned}
\mathrm{q} \omega(\bar{\xi}) \theta(\bar{\eta}) & =\mathrm{q} \omega(\bar{\xi}) \mathrm{q}^{-1}(\eta)=p \cdot g \omega(\bar{\xi})(\mathrm{p} \cdot g)^{-1}(\eta)=p g \omega(\bar{\xi}) g^{-1} p^{-1}(\eta) \\
& =\operatorname{pAd}_{g}(\omega(\bar{\xi})) \theta(\bar{\eta})=p \omega\left(D_{g^{-1}} \bar{\xi}\right) \theta(\bar{\eta})=p \omega(\tilde{\xi}) \theta(\bar{\eta}) \\
& =p \omega(\bar{\xi}) \theta(\bar{\eta})
\end{aligned}
$$

where we have used the equivariance of $\omega, R_{g}^{*} \omega=A d_{g^{-1}} \omega$, and the fact that $D R_{g}$ maps lifts into lifts, since $\pi \circ R_{g}=\pi$ and therefore $D \pi \circ D R_{g}=D \pi$ for all $g \in G l(m)$. By abuse of notation $\bar{\eta}$ denotes a lift to both $q$ and $p$ in $\operatorname{TFr}_{\mathrm{Gl}}(\mathrm{m})$.

Note that we have only needed $\mathbf{G l}(\mathrm{m})$ for the fact that $\operatorname{Ad}_{g}(\xi)=g \xi g^{-1}$, so this will be true for all principal bundles in this work, if we adjust the vector bundle in which the sections are taken.

The inverse map, sending a section to a form on the principal bundle is defined by

$$
\omega(s)(\xi)=p^{-1} s(D \pi(\xi)) p,
$$

where $p \in \operatorname{Fr}_{\mathrm{Gl}}(M)$ is some frame, $\xi \in \mathrm{T}_{\mathrm{p}} \mathrm{Fr}_{\mathrm{Gl}}(M)$ and $s \in \Gamma\left(\mathrm{T}^{*} \mathrm{M} \otimes \operatorname{End}(\mathrm{TM})\right)$ is the section. This form is clearly a horizontal $\mathfrak{g l}(\mathrm{m})$-valued one-form. It is also equivariant because

$$
\begin{aligned}
R_{g}^{*} \omega(s)(\xi) & =(p g)^{-1} s\left(D \pi \circ D R_{g}(\xi)\right) p g=g^{-1} p^{-1} s(D \pi(\xi)) p g \\
& =\operatorname{Ad}_{g^{-1}} \omega(s)(\xi) .
\end{aligned}
$$

It is easy to show that these two maps are inverse of each other, which concludes the proof.

Definition 3.12 (Corresponding forms). As denoted in the proof above, the section of $T^{*} M \otimes \operatorname{End}(T M)$ corresponding to $\omega$ is denoted by $s(\omega)$, and the form corresponding to a section $s$ by $\omega(s)$.

Note that this result remains true for reductions of the basis bundle, if we adjust the vector bundle in which the sections are taken. For example, the above mentioned forms on $\mathrm{Fr}_{S O}(M)$ correspond to sections in $T^{*} M \otimes \mathfrak{s o}(T M)$ and the forms on $\operatorname{Fr}_{S p}(M)$ to sections of $T^{*} M \otimes \mathfrak{s p}(T M)$.

Example 3.13 (Difference form). A well known example of this correspondence is between the difference form of two connections on a principal bundle, and the difference tensor of the two associated covariant derivatives. This follows immediately from equation $3 \cdot 38$ ). 


\subsection{Setting}

We will recover the results from [GN92] for principal bundles.

Let $(M, g)$ be an Riemannian manifold of dimension $4 m \in \mathbb{N}$, and let $M \curvearrowleft G$ be a tri-hamiltonian action of $G$ on $M$. Let $k \in \mathbb{N}$ be the dimension of the Lie group $G$. We denote the momentum map by $\mu: M \rightarrow \mathbb{R}^{3} \otimes \mathfrak{g}^{*}$. We assume that $0 \in \mathbb{R}^{3} \otimes \mathfrak{g}^{*}$ is a regular value of $\mu$. This implies that $G$ acts on the submanifold $\mu^{-1}(0)$, because equation (3.3) guarantees that for $x \in \mu^{-1}(0)$, i.e. $\mu_{A}(x)=0$ for all $A$, we have

$$
\mu_{\mathrm{A}}(\mathrm{x} . \mathrm{h})=\operatorname{Ad}_{\mathrm{h}}^{*} \circ \mu_{\mathrm{A}}(\mathrm{x})=0, \quad \forall \mathrm{h} \in \mathrm{G},
$$

and hence $x . h \in \mu^{-1}(0)$.

We assume further that this action is free and proper, so that the quotient $\mu^{-1}(0) / G$ is a Hausdorff space, and define $N:=\mu^{-1}(0) / G$.

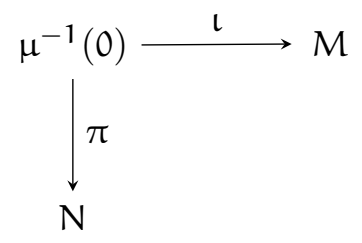

We will show that $\mathrm{N}$ also is a hyperkähler manifold, and that the second fundamental form of $\mu^{-1}(0)$ in $M$ is given by the Hessian of $\mu$, compare [GN92] and [Hit+87].

\subsubsection{The Splitting of TM}

The tri-hamiltonian action $M \curvearrowleft G$ splits the vector bundle TM over $\mu^{-1}(0)$, i.e. the ambient bundle

$$
\text { เ*TM, }
$$

in the following way.

Proposition 3.14. If $x \in \mu^{-1}(0)$, we have

$$
\mathrm{T}_{x} \mathrm{M}=\mathrm{T}_{x} \mu^{-1}(0) \oplus \mathrm{T}_{x} \mu^{-1}(0)^{\perp}=\mathrm{H}_{x} \oplus \mathfrak{g} \oplus \mathrm{T}_{x} \mu^{-1}(0)^{\perp},
$$

where $\mathfrak{g} \subset \mathrm{T}_{x} \mathrm{M}$ is defined by the fundamental vector fields, i.e. the image of $\mathrm{K}: \mathfrak{g} \rightarrow \Gamma(\mathrm{TM})$, and $\mathrm{H}_{\mathrm{x}}$ is the orthogonal complement to $\mathfrak{g}$ in $\mathrm{T \mu}^{-1}(0)$ with respect to the metric g. All direct sums are orthogonal.

Then $\mathrm{H}_{\mathrm{x}}$ is a quaternionic subspace of $\mathrm{T}_{\mathrm{x}} \mathrm{M}$ and

$$
\mathrm{T}_{x} \mu^{-1}(0)^{\perp}=\mathrm{Ig} \oplus \mathrm{Jg} \oplus \mathrm{K} \mathfrak{g} .
$$


Proof. If $\xi \in \mathfrak{g}$ and $\eta \in \mathrm{T}_{x} \mu^{-1}(0)$ then $\eta$ is tangent to a level set of $\mu$, i.e. $\mathrm{d} \mu(\eta)=0$, which implies for $A \in\{I, J, K\}$

$$
g\left(A K^{\xi}, \eta\right)=\omega_{A}\left(K^{\xi}, \eta\right)=\left\langle\xi, \mathrm{d} \mu_{A}(\eta)\right\rangle=0,
$$

hence $A K^{\xi} \in T \mu^{-1}(0)^{\perp}$ for all $A$.

Furthermore the sets $I \mathfrak{g}$, Jg and $\mathrm{Kg}$ have a trivial intersection. Indeed, assume $\xi, \eta \in$ $\mathfrak{g}$ with $\mathrm{I} \xi=\mathrm{J} \eta$. Then $\mathrm{K} \xi=\eta$ but since $\mathrm{K} \xi$ is in $\mathrm{T} \mu^{-1}(0)^{\perp}, \eta=\xi=0$.

Since the codimension of $\mu^{-1}(0)$ in $M$ is $3 k$, where $k=\operatorname{dim} G=\operatorname{dim} \mathfrak{g}$, we see that

$$
\mathrm{T}_{x} \mu^{-1}(0)^{\perp}=\mathrm{I} \mathfrak{g} \oplus \mathrm{Jg} \oplus \mathrm{K} \mathfrak{g} .
$$

Finally, I, J and $\mathrm{K}$ let the orthogonal complement of $\mathrm{H}_{\mathrm{x}}$ invariant and are orthogonal, so they also let $\mathrm{H}_{x}$ invariant.

We conclude that TM splits over $\mu^{-1}(0)$ into two quaternionic sub-bundles

$$
\mathrm{TM}=\mathrm{H} \oplus \mathfrak{g} \otimes_{\mathbb{R}} \mathbb{H} .
$$

Notice that while the first bundle has a quaternionic structure, the second one has a quaternionic and a real structure. This will become important later on.

The metric $g$ of $M$ induces a metric on $H$. Since $M \curvearrowleft G$ is hyperkähler and $g$ is G-invariant it furnishes $\mathrm{N}$ with a Riemannian metric. Similarly the quaternionic structure on $\mathrm{M}$ induces one on $\mathrm{H}$ (because of the quaternionic decomposition above), which in turn induces one on $\mathrm{N}$ compatible with the metric. This reduces the principal bundle of orthogonal frames on $N$ to the structure group $\operatorname{Sp}(n)(n=m-k, 4 n$ is the dimension of $N$ ). We will show later that the connection of $N$ reduces so that $N$ is indeed a hyperkähler manifold.

\subsubsection{The Principal Bundles}

Similar to the vector bundle TM, we may depict the splitting in the principal bundle setting. Fix a splitting

$$
\mathbb{R}^{4 m}=\mathbb{R}^{4 n} \oplus \mathbb{R}^{k} \oplus \mathbb{R}^{3 k}=\mathbb{H}^{n} \oplus \mathbb{H}^{k}
$$

Now we can ask frames $p: \mathbb{R}^{4 m} \rightarrow T_{\chi} M$ to respect various degrees of the structure. Let $x \in \mu^{-1}(0)$.

- $p: \mathbb{R}^{4 m} \rightarrow \mathrm{T}_{x} \mathrm{M}$ with no condition at all. These frames are in the pull back of the frame bundle $\operatorname{Fr}_{S O}(M)$ to $\mu^{-1}(0)$, denoted by $\imath^{*} \mathrm{Fr}_{S O}(M)$. 
- $p: \mathbb{R}^{4 m} \rightarrow \mathrm{T}_{x} M$ with $p\left(\mathbb{R}^{4 n} \oplus \mathbb{R}^{k}\right)=T_{\mu}^{-1}(0)$, frames adapted to the submanifold $\mu^{-1}(0) \subset M$. This is a principal bundle whose structure group is $\mathbf{S O}(4 n+k) \times$ $\mathbf{S O}(3 \mathrm{k})$, corresponding to the possible rotations of the frame in $T \mu^{-1}(0)$ and $\mathrm{T} \mu^{-1}(0)^{\perp}$. We denote it by

$$
\operatorname{Fr}_{S O}\left(\mu^{-1}(0), M\right)=\left\{p \in \operatorname{Fr}_{S O}(M): \quad \operatorname{im}\left(\left.p\right|_{\mathbb{R}^{4 n+k}}\right)=\mathrm{T}^{-1}(0)\right\},
$$

- $p: \mathbb{R}^{4 n} \oplus \mathbb{R}^{k} \rightarrow T_{x} \mu^{-1}(0)$. These frames can be identified with frames of $\mu^{-1}(0)$. We denote them with $\operatorname{Fr}_{S O}\left(\mu^{-1}(0)\right)$.

- $p: \mathbb{R}^{4 n} \oplus \mathbb{R}^{k} \rightarrow \mathrm{T}_{x} \mu^{-1}(0)$ with $p\left(\mathbb{R}^{4 n}\right)=\mathrm{H}_{x}$. These frames are frames of $\mu^{-1}(0)$ adapted to the fibration $\pi: \mu^{-1}(0) \rightarrow N$. The principal bundle of these have structure group $\mathbf{S O}(4 n) \times \mathbf{S O}(k)$ corresponding to the rotations in the fiber and its orthogonal complement. We denote the bundle by

$$
\operatorname{Fr}_{S O}\left(N, \mu^{-1}(0)\right)=\left\{p \in \operatorname{Fr}_{S O}\left(\mu^{-1}(0)\right): \quad \operatorname{im}\left(\left.p\right|_{\mathbb{R}^{4 n}}\right)=H_{x}\right\},
$$

- $p: \mathbb{R}^{4 n} \rightarrow H_{x}$. The principal bundle of these frames can be identified with the pull back of $\operatorname{Fr}_{\mathrm{SO}}(\mathrm{N})$ to $\mu^{-1}(0)$ (note that we know already that $\mathrm{N}$ is a Riemannian manifold). We denote it by $\pi^{*} \mathrm{Fr}_{\mathrm{SO}}(\mathrm{N})$.

We may restrict the principal bundles above to quaternionic frames where it makes sense. Fix

$$
\mathbb{H}^{m}=\mathbb{H}^{n} \oplus \mathbb{H}^{k}
$$

respecting (3.21). This induces the following bundles, where all frames are $\mathbb{H}$-linear.

- $p: \mathbb{H}^{m} \rightarrow \mathrm{T}_{x} M$ are the frames that make up the pull back of

$$
\operatorname{Fr}_{S p}(M)=\left\{p \in \operatorname{Fr}_{S O}(M): p \text { is } \mathbb{H} \text {-linear }\right\}
$$

to $\mu^{-1}(0)$. It is naturally a reduction of $\iota^{*} \operatorname{Fr}_{S O}(M)$ to quaternionic frames, has structure group $S p(m)$ and will be denoted by $\iota^{*} \operatorname{Fr}_{S p}(M)$.

- $p: \mathbb{H}^{m} \rightarrow \mathrm{T}_{x} M$ with $p\left(\mathbb{H}^{n}\right)=\mathrm{H}_{x}$ and $p\left(\mathbb{H}^{k}\right)=\mathfrak{g} \otimes \mathbb{H}$ respecting both the quaternionic and real structure. We denote this principal bundle with structure group $\mathbf{S p}(n) \times \mathbf{S O}(k)$ by

$$
\operatorname{Fr}_{S p}(N, M)=\left\{p \in \imath^{*} \operatorname{Fr}_{S p}(M): \operatorname{im}\left(\left.p\right|_{\mathbb{H}^{n}}\right)=H_{x}, \operatorname{im}\left(\left.p\right|_{\mathbb{H}^{k}}\right)=\mathfrak{g} \otimes \mathbb{H}\right\}
$$

The frames are adapted to the quaternionic splitting of $\mathrm{T}_{x} \mathrm{M}=\mathrm{H}_{x} \oplus \mathfrak{g} \otimes \mathbb{H}$ and respect the real structure of the second, $p\left(\operatorname{Re}\left(\mathbb{H}^{k}\right)\right)=\operatorname{Re}(\mathfrak{g} \otimes \mathbb{H})=\mathfrak{g}$, so in particular (because I, J, K are orthogonal) respect the splitting $T \mu^{-1}(0) \oplus T \mu^{-1}(0)^{\perp}$. 
- $p: \mathbb{H}^{n} \rightarrow H_{x}$ are the frames of the pulled back bundle $\operatorname{Fr}_{S p}(N)$ to $\mu^{-1}(0)$ and is denoted by $\pi^{*} \operatorname{Fr}_{S p}(\mathrm{~N})$.

There are plenty of natural maps between these bundles. We will be using the following.

- Reductions to quaternionic frames, denoted by $i$ : Some of the real frame bundles can be reduced to quaternionic frames, which induces maps from the quaternionic world to the real world. This is obviously the case for $\operatorname{Fr}_{S p}(M) \rightarrow \operatorname{Fr}_{S O}(M)$, $\mathrm{Fr}_{\mathrm{Sp}}(\mathrm{N}) \rightarrow \mathrm{Fr}_{\mathrm{SO}}(\mathrm{N})$ and their pull backs to $\mu^{-1}(0)$. Finally this is also the case for $\operatorname{Fr}_{S p}(N, M) \rightarrow \operatorname{Fr}_{S O}\left(\mu^{-1}(0), M\right)$, because a quaternionic frame that respects the splitting $T_{x} \mu^{-1}(0) \oplus T_{x} \mu^{-1}(0)^{\perp}$, automatically respects the quaternionic splitting $\mathrm{H}_{x} \oplus \mathfrak{g} \otimes \mathbb{H}$, as can be seen by applying one of the complex structures to $\mathrm{T}_{x} \mu^{-1}(0)^{\perp}$. In other words, $(\mathbf{S O}(4 n+k) \times \mathbf{S O}(3 k)) \cap \mathbf{S p}(m) \cong \mathbf{S p}(n) \times \mathbf{S O}(k)$.

- Reduction to more structured frames, denoted by $j$ : Some of the bundles are simply restrictions of other bundles to frames respecting more structures. This is the case for

$$
\operatorname{Fr}_{S O}\left(\mu^{-1}(0), M\right) \rightarrow \iota^{*} \operatorname{Fr}_{S O}(M), \quad \operatorname{FrSO}_{S O}\left(N, \mu^{-1}(0)\right) \rightarrow \operatorname{Fr}_{S O}\left(\mu^{-1}(0)\right)
$$

and

$$
\operatorname{Fr}_{S p}(N, M) \rightarrow \imath^{*} \operatorname{Fr}_{S p}(M)
$$

- Induced maps by pull backs, also denoted by j: There are of course canonical maps $\iota^{*} \mathrm{Fr}_{\mathrm{SO}}(\mathrm{M}) \rightarrow \mathrm{Fr}_{\mathrm{SO}}(\mathrm{M})$ and similar for $\pi: \mu^{-1}(0) \rightarrow \mathrm{N}$ and the quaternionic bundles.

- Restrictions of frames, denoted by $k$ : Some bundles allow natural projections to other bundles by restricting the frame to a subspace of its domain. This is the case for

$$
\operatorname{Fr}_{\mathrm{SO}}\left(\mu^{-1}(0), M\right) \rightarrow \operatorname{Fr}_{\mathrm{SO}}\left(\mu^{-1}(0)\right), \quad \mathrm{Fr}_{\mathrm{SO}}\left(\mathrm{N}, \mu^{-1}(0)\right) \rightarrow \pi^{*} \mathrm{Fr}_{\mathrm{SO}}(\mathrm{N})
$$

and

$$
\mathrm{Fr}_{\mathrm{Sp}}(\mathrm{N}, \mathrm{M}) \rightarrow \pi^{*} \mathrm{Fr}_{\mathrm{Sp}}(\mathrm{N}), \quad \mathrm{Fr}_{\mathrm{Sp}}(\mathrm{N}, \mathrm{M}) \rightarrow \mathrm{Fr}_{\mathrm{SO}}\left(\mathrm{N}, \mu^{-1}(0)\right)
$$

The aforementioned bundles are depicted in the following diagram. 


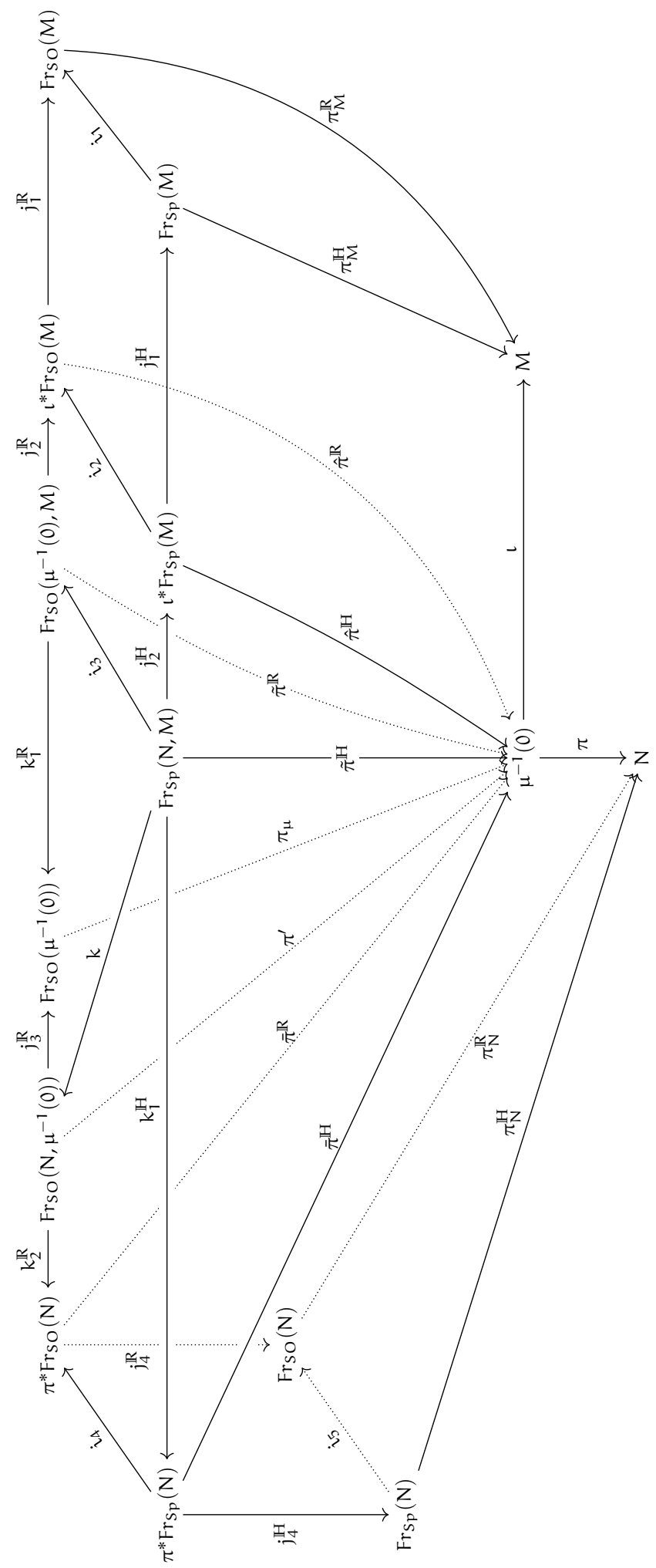




\subsection{The Induced Connections}

In this section we will start with the Levi Civita connection on $\operatorname{Fr}_{S O}(M)$ and chase it through the diagram. This will show that $\mathrm{N}$ is indeed a hyperkähler manifold and recover the results from [GN92].

\subsubsection{Forms on $\operatorname{Fr}_{S p}(M)$}

Starting with the solder form $\theta^{M, \mathbb{R}}$ and the Levi Civita connection $\varphi^{M, \mathbb{R}}$ on $\operatorname{Fr}_{S O}(M)$, we first induce the forms $\theta^{M, H}$ and $\varphi^{M, \mathbb{H}}$ on $\operatorname{Fr}_{S p}(M)$, by pulling back with $i_{1}$,

$$
\theta^{M, H}=i_{1}^{*} \theta^{M, \mathbb{R}}, \quad \varphi^{M, H}=i_{1}^{*} \varphi^{M, \mathbb{R}} .
$$

Since $M$ is a hyperkähler-manifold, $\varphi^{M, H}$ is a connection on $\operatorname{Fr}_{S p}(M)$ satisfying the pulled back structure equation

$$
\mathrm{d} \theta^{\mathrm{M}, \mathbb{H}}+\varphi^{\mathrm{M}, \mathbb{H}} \wedge \theta^{\mathrm{M}, \mathbb{H}}=0 .
$$

As remarked in 3.9 $\theta^{M, \mathbb{H}}$ is again the soldering form of $\operatorname{Fr}_{S p}(M)$, hence $\varphi^{M, \mathbb{H}}$ is a torsion free connection on $\mathrm{Fr}_{S p}(M)$.

\subsubsection{Forms on $\iota^{*} \mathrm{Fr}_{\mathrm{SO}}(\mathrm{M})$ and ${ }^{*} \mathrm{Fr}_{\mathrm{Sp}}(\mathrm{M})$}

The solder forms and connection forms on $\operatorname{Fr}_{S O}(M)$ and $\operatorname{Fr}_{S p}(M)$ further induce connections on the ambient principal bundles $\iota^{*} \operatorname{Fr}_{S p}(M)$ and $\iota^{*} \operatorname{Fr}_{S O}(M)$ which we will denote by $\hat{\varphi}^{\mathbb{R}}, \hat{\theta}^{\mathbb{R}}$ and $\hat{\varphi}^{\mathbb{H}}, \hat{\theta}^{\mathbb{H}}$ with the obvious choice. The $\hat{\varphi}$ are connections, since we do not change the fibers of the principal bundle (although some may be discarded). It is also a torsion free connection, since the structural equation $\mathrm{d} \theta+\varphi \wedge \theta=0$ survives the pull back and by using remark (3.9), the pulled back solder forms are natural

$$
\hat{\theta}_{p}^{\mathbb{R}}(\xi)=p^{-1} \circ D \hat{\pi}_{p}^{\mathbb{R}}(\xi), \quad \hat{\theta}_{\mathbf{q}}^{\mathbb{H}}(\eta)=q^{-1} \circ D \hat{\pi}_{q}^{\mathbb{H}}(\eta),
$$

where $p \in \iota^{*} \operatorname{Fr}_{S O}(M), \xi \in T_{p} \iota^{*} \operatorname{Fr}_{S O}(M)$ and $q \in \iota^{*} \operatorname{Fr}_{S p}(M), \eta \in T_{q} \iota^{*} \operatorname{Fr}_{S p}(M)$.

\subsubsection{Forms on $\operatorname{Fr}_{\mathrm{SO}}\left(\mu^{-1}(0), M\right)$}

The next step is to transfer these forms to the principal bundle

$$
\operatorname{Fr}_{S O}\left(\mu^{-1}(0), M\right)=\left\{p \in \iota^{*} \operatorname{Fr}_{S O}(M): \operatorname{im}\left(\left.p\right|_{\mathbb{R}^{4 n+k}}\right)=\mathrm{T}^{-1}(0)\right\},
$$

which has structure group $\mathbf{S O}(4 n+k) \times \mathbf{S O}(3 k)$.

Different to before is that $\operatorname{Fr}_{S O}\left(\mu^{-1}(0), M\right)$ is in general not horizontal in the ambient bundle, hence we need to project in order to get a connection. 
Lemma (3.7) allows us to define connections on the adapted frame bundles given by $\operatorname{Fr}_{S O}\left(\mu^{-1}(0), M\right)$ and $\operatorname{Fr}_{S p}(N, M)$. With the inclusion

$$
i: \mathbf{S O}(4 n+k) \times \mathbf{S O}(3 k) \rightarrow \mathbf{S O}(4 m), \quad(A, B) \mapsto\left(\begin{array}{cc}
A & 0 \\
0 & B
\end{array}\right)
$$

we get the Lie algebra decomposition (as vector spaces)

$$
\mathfrak{s o}(4 m)=\mathfrak{s o}(4 n+k) \oplus \mathfrak{s o}(3 k) \oplus \mathfrak{f},
$$

where

$$
\mathfrak{f}=\left\{\left(\begin{array}{cc}
0 & C \\
-C^{t} & 0
\end{array}\right) \in \mathfrak{s o}(4 m): C \in \operatorname{Mat}(4 n+k, 3 k)\right\} .
$$

If $A \in \operatorname{im}(i)$ and $\xi \in \mathfrak{f}$, then $\operatorname{Ad}_{A}(\xi)=A \xi A^{-1} \in \mathfrak{f}$, hence we have a connection $\tilde{\varphi}^{\mathbb{R}}=\operatorname{pr}_{\mathfrak{s o}(4 n+k) \oplus \mathfrak{s o}(3 k)} \circ j_{2}^{\mathbb{R} *} \hat{\varphi}^{\mathbb{R}}$ on $\operatorname{Fr}_{S O}\left(\mu^{-1}(0), M\right)$. This connection naturally decomposes into two equivariant one-forms $\phi_{1}^{\mathbb{R}}$ and $\phi_{2}^{\mathbb{R}}$ with values in $\mathfrak{s o}(4 n+k)$ and $\mathfrak{s o}(3 k)$ respectively.

We can go ahead and extend $\tilde{\varphi}^{\mathbb{R}}$ back to ${ }^{*} \operatorname{Fr}_{\mathrm{SO}}(M)$, which gives us a connection $\hat{\varphi}^{\prime \mathbb{R}}$. The difference form

$$
\hat{\tau}^{\mathbb{R}}=\hat{\varphi}^{\mathbb{R}}-\hat{\varphi}^{\prime \mathbb{R}}
$$

is a equivariant horizontal one form, hence the pull back

$$
\tau^{\mathbb{R}}=j_{2}^{\mathbb{R} *} \hat{\tau}^{\mathbb{R}}=j_{2}^{\mathbb{R} *} \hat{\varphi}^{\mathbb{R}}-\tilde{\varphi}^{\mathbb{R}}
$$

is also.

The induced connection $\tilde{\varphi}^{\mathbb{R}}$ is torsion free, since $\tilde{\theta}^{\mathbb{R}}$, the pull back of the solder form, is again the solder form on $\mathrm{Fr}_{\mathrm{SO}}\left(\mu^{-1}(0), \mathrm{N}\right)$. We pull back the structure equation $d \hat{\theta}^{\mathbb{R}}+\hat{\varphi}^{\mathbb{R}} \wedge \hat{\theta}^{\mathbb{R}}=0$ to get

$$
d \tilde{\theta}^{\mathbb{R}}+\left(j_{2}^{\mathbb{R} *} \hat{\varphi}^{\mathbb{R}}\right) \wedge \tilde{\theta}^{\mathbb{R}}=d \tilde{\theta}^{\mathbb{R}}+\left(\tilde{\varphi}^{\mathbb{R}}+\tau^{\mathbb{R}}\right) \wedge \tilde{\theta}^{\mathbb{R}}=0 .
$$

Since $\tilde{\theta}^{\mathbb{R}}$ has values in $\mathbb{R}^{4 n+k}$, we can split the equation into the following two equations

$$
\begin{aligned}
\mathrm{d} \tilde{\theta}^{\mathbb{R}}+\tilde{\varphi}^{\mathbb{R}} \wedge \tilde{\theta}^{\mathbb{R}}=0, \\
\tau^{\mathbb{R}} \wedge \tilde{\theta}^{\mathbb{R}}=0,
\end{aligned}
$$

which shows that $\tilde{\varphi}^{\mathbb{R}}$ is indeed torsion free.

$\tau^{\mathbb{R}}$ splits naturally into two forms with values in the top right matrices and bottom left matrices. Let $\tau_{1}^{\mathbb{R}}$ denote the one that has values in the bottom left. Hence we have the splitting 


$$
j_{2}^{\mathbb{R} *} \hat{\varphi}^{\mathbb{R}}=\left(\begin{array}{cc}
\phi_{1}^{\mathbb{R}} & -\left(\tau_{1}^{\mathbb{R}}\right)^{\mathrm{t}} \\
\tau_{1}^{\mathbb{R}} & \phi_{2}^{\mathbb{R}}
\end{array}\right) .
$$

Using lemma 3.10 to identify $\tau_{1}^{\mathbb{R}}$ with a $(2,1)$-tensor on $\mu^{-1}(0)$, via

$$
s\left(\tau_{1}^{\mathbb{R}}\right)(\xi, \eta)=p \tau_{1}^{\mathbb{R}}(\bar{\xi}) \tilde{\theta}^{\mathbb{R}}(\bar{\eta}),
$$

where $p$ is a frame in $F\left(\mu^{-1}(0), M\right)$ and $\bar{\xi}, \bar{\eta}$ are lifts (compare lemma $\left.\sqrt{3.10}\right)$ ).

Proposition 3.15 (Second fundamental form). $s\left(\tau_{1}^{\mathbb{R}}\right)$ is the second fundamental form of $\mu^{-1}(0)$ in $M$.

Proof. In the next subsection we will show that $\phi_{1}^{\mathbb{R}}$ is the pull back of the Levi Civita connection on $\mathrm{Fr}_{\mathrm{SO}}\left(\mu^{-1}(0)\right)$. The covariant derivative of a connection $\varphi$ with soldering form $\theta$ is given by

$$
\nabla_{\mathrm{t}} \mathrm{X}=\mathrm{p}\left(\overline{\mathrm{t}} \theta(\overline{\mathrm{X}})+\varphi(\overline{\mathrm{t}}) \theta\left(\overline{\mathrm{X}}_{\mathrm{p}}\right)\right),
$$

where $\bar{t}$ and $\bar{X}$ are lifts of the tangent vector $t$ and vector field $X$ to a frame $p$ (see e.g. [BC64, p. 6.4], but note that this book has a very unusual sign convention for the second fundamental form). Hence the second fundamental form is given by

$$
\begin{aligned}
\mathrm{II}(X, Y) & =\nabla_{X}^{M} Y-\nabla_{X}^{\mu^{-1}(0)} Y=p\left(j_{2}^{\mathbb{R} *} \hat{\varphi}^{\mathbb{R}}(\bar{X})-\phi_{1}^{\mathbb{R}}(\bar{X})\right) \tilde{\theta}^{\mathbb{R}}(\bar{Y}) \\
& =p\left(j_{2}^{\mathbb{R} *} \hat{\varphi}^{\mathbb{R}}(\bar{X})-\tilde{\varphi}^{\mathbb{R}}(\bar{X})\right) \tilde{\theta}^{\mathbb{R}}(\bar{Y})=p \tau^{\mathbb{R}}\left(\bar{X}_{\mathfrak{p}}\right) \tilde{\theta}^{\mathbb{R}}\left(\bar{Y}_{p}\right) .
\end{aligned}
$$

Here we have used that $X$ and $Y$ are tangent to $\mu^{-1}(0)$ and hence $\phi_{2}^{\mathbb{R}}\left(\bar{X}_{p}\right) \tilde{\theta}^{\mathbb{R}}\left(\bar{Y}_{p}\right)=0$. Note that II is symmetric, because $\tau^{\mathbb{R}} \wedge \tilde{\theta}^{\mathbb{R}}=0$, by equation (3.35). Since the second fundamental form is only defined for tangent vectors to $\mu^{-1}(0)$ and takes values orthogonal to $\mu^{-1}(0)$, we have to restrict $\tau^{\mathbb{R}}$ to $\tau_{1}^{\mathbb{R}}$ as described above.

Proposition 3.16 (Second fundamental form as Hessian). Let $\mathrm{f}: \mathrm{M} \rightarrow \mathrm{V}$ be a smooth map, where $\mathrm{M}$ is a Riemannian manifold and $\mathrm{V}$ a vector space. Assume further, that $\mathrm{O} \in \mathrm{V}$ is a regular value. $\mathrm{Df}: \mathrm{TM} \rightarrow \mathrm{V}$ identifies every fiber of the bundle $\mathrm{Tf}^{-1}(0)^{\perp}$ with $\mathrm{V}$, and under this identification the negative of the Hessian matrix of $\mathrm{f}$ equals the second fundamental form of $\mathrm{f}^{-1}(0)$ in $\mathrm{M}$.

Proof. The first claim is just the dimension formula for a linear map,

$$
D f_{p}: \operatorname{Tf}^{-1}(0) \oplus \operatorname{Tf}^{-1}(0)^{\perp} \rightarrow V,
$$

which has kernel $\mathrm{Tf}^{-1}(0)$. Note that the second equality only holds for vector fields tangent to $f^{-1}(0)$, since the second fundamental form is only defined for these. Let $X$ 
and $Y$ be vector fields tangent to $f^{-1}(0)$. Then

$$
\begin{aligned}
\operatorname{Hess}(f)(X, Y) & =X(Y f)-\operatorname{Df}\left(\nabla_{X}^{M} Y\right) \\
& =X(\underbrace{\operatorname{Df}(Y)}_{=0})-\underbrace{\operatorname{Df}\left(\nabla_{X}^{\mu^{-1}(0)} Y\right)}_{=0}-\operatorname{DfII}(X, Y) \\
& =-\operatorname{DfII}(X, Y)
\end{aligned}
$$

In this sense, $\tau_{1}^{\mathbb{R}}$ is associated with the $-\operatorname{Hess}(\mu)$ by the two aforementioned propositions.

\subsubsection{Forms on $\operatorname{Fr}_{\mathrm{SO}}\left(\mu^{-1}(0)\right)$}

Recall that the torsion free connection $\tilde{\varphi}^{\mathbb{R}}$ decomposes into two one forms $\phi_{1}^{\mathbb{R}}$ and $\phi_{2}^{\mathbb{R}}$. $\phi_{1}^{\mathbb{R}}$ with values in $\mathfrak{s o}(4 n+k)$ induces a connection on $\operatorname{Fr}_{S O}\left(\mu^{-1}(0)\right)$, because

$$
\begin{aligned}
\phi_{1}^{\mathbb{R}}\left(\left(D k_{1}^{\mathbb{R}}\right)^{-1}(0)\right) & =0, \\
\mathrm{R}_{g}^{*} \phi_{1}^{\mathbb{R}} & =\phi_{1}^{\mathbb{R}} \quad \forall \mathrm{g} \in \mathrm{O}(3 \mathrm{k}) \subset \mathrm{O}(4 \mathrm{~m}),
\end{aligned}
$$

which is true because $\mathrm{Dk}_{1}^{\mathbb{R}}: \mathfrak{s o}(4 n+k) \oplus \mathfrak{s o}(3 k) \rightarrow \mathfrak{s o}(4 n+k)$ is the projection. It allows us to define

$$
\varphi^{\mu^{-1}(0)}(\eta)=\phi_{1}^{\mathbb{R}}(\tilde{\eta}), \quad \tilde{\eta} \in\left(D k_{1}^{\mathbb{R}}\right)_{q}^{-1}(\eta),
$$

i.e. $k_{1}^{\mathbb{R} *} \varphi^{\mu^{-1}(0)}=\phi_{1}^{\mathbb{R}}$. Since the solder form on $\mu^{-1}(0)$ pulled back to $F\left(\mu^{-1}(0), M\right)$ is the form $\tilde{\theta}^{\mathbb{R}}$, we get the equation

$$
\mathrm{d} \theta^{\mu^{-1}(0), \mathbb{R}}+\varphi^{\mu^{-1}(0)} \wedge \theta^{\mu^{-1}(0), \mathbb{R}}=0,
$$

and see that $\varphi^{\mu^{-1}(0)}$ is the unique Levi Civita connection on $\mu^{-1}(0)$.

\subsubsection{Riemannian Submersions}

The next step involves understanding Riemannian submersions on the level of frame bundles. Since there is no exposition of this known to the author, we will describe it in a general setting, and apply it to the reduction afterwards.

Let us at this point recall the basics of the Riemannian submersion theory of $\mathrm{O}^{\prime}$ Neill $\left[\mathrm{O}^{\prime} \mathrm{N} 66\right]$. A Riemannian submersion $\pi: \mathrm{M}^{\mathrm{m}} \rightarrow \mathrm{B}^{\mathrm{b}}$ is a smooth map between two Riemannian manifolds such that $\pi$ is a submersion and $\left.D \pi_{x}\right|_{H_{x}}: H_{x} \rightarrow T_{\pi(x)} B$ is a isometry for all $x \in M$, where $H_{x}$ is the orthogonal complement of $\operatorname{ker}(D \pi) \subset T_{x} M$.

To such a Riemannian submersion we may associate two important $(2,1)$-tensor fields on $M$, 


$$
\begin{aligned}
\mathrm{T}_{X} \mathrm{Y} & =\mathcal{H} \nabla_{\mathcal{V} X}^{M} \mathcal{V} Y+\mathcal{V} \nabla_{\mathcal{V} X}^{M} \mathcal{H} Y \\
A_{X} Y & =\mathcal{H}_{\mathcal{H}_{X}}^{M} \mathcal{V} Y+\mathcal{V} \nabla_{\mathcal{H} X}^{M} \mathcal{H} Y,
\end{aligned}
$$

where $\mathcal{H}$ and $\mathcal{V}$ are the horizontal and vertical projection in $T M$, respectively. $T$ is known to be the second fundamental form of each fiber (if vertical vector fields are plugged in), whereas $A$ is related to the obstruction to integrability of the horizontal distribution on $M$. An important fact is that

$$
A_{X} Y=\frac{1}{2} v[X, Y],
$$

for horizontal vector fields $X$ and $Y$. If the Riemannian submersion $\pi: M \rightarrow B$ should also happen to be a principal bundle, and we fix the connection corresponding to the horizontal subspaces, then $2 A_{X} Y=-R(X, Y)$, where $R(X, Y)$ is the curvature of the connection, if we identify the vertical tangent space with the Lie algebra as usual.

In the world of principal bundles this can be expressed the following way. Let $\operatorname{Fr}(M)$ be the principal bundle of frames and $\operatorname{Fr}(B, M)$ the reduction to adapted frames on $M$. Here a frame is adapted if it respects the splitting of TM into horizontal and vertical parts, i.e.

$$
\operatorname{Fr}(B, M)=\left\{p \in \operatorname{Fr}(M): \operatorname{im}\left(\left.p\right|_{\mathbb{R}^{\mathrm{b}}}\right) \text { is horizontal }\right\} \text {. }
$$

Then a pull back of the Levi Civita connection $\phi$ on $\operatorname{Fr}(M)$ and the solder form $\theta$ gives, after a suitable projection, a connection $\psi$ on $\operatorname{Fr}(B, M)$ with structure equation

$$
\mathrm{d} \theta^{\prime}+\psi \wedge \theta^{\prime}+\tau \wedge \theta^{\prime}=0,
$$

where $\theta^{\prime}$ is the pull back of the solder form, $\psi$ the projected connection and $\tau=$ $i^{*} \phi-\psi$, where $i: \operatorname{Fr}(B, M) \rightarrow \operatorname{Fr}(M)$ is the inclusion. We see that $\tau$ is an obstruction to the integrability of the horizontal distribution, because for a product manifold $M=$ $M_{1} \times M_{2}$ we have the commutative diagram

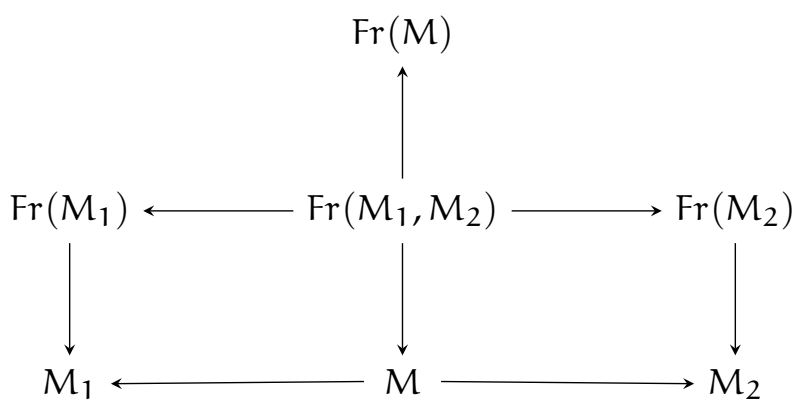


and the connection on $\operatorname{Fr}(M)$ reduces to a connection on $\operatorname{Fr}\left(M_{1}, M_{2}\right)$, which is the sum of the connections pulled back from $\operatorname{Fr}\left(M_{i}\right)$. On the other hand, from the construction of the last section, we also know that $\tau$ is related to the second fundamental forms of the fibers.

The notion of horizontal and vertical projection extends to horizontal forms on $\operatorname{Fr}(B, M)$, via

$$
\begin{aligned}
& \tau_{h}(\xi)=\tau\left(\overline{\mathcal{H} \mathrm{D} \pi^{\prime}(\xi)}\right) \\
& \tau_{v}(\xi)=\tau\left(\overline{\mathcal{V D} \pi^{\prime}(\xi)}\right),
\end{aligned}
$$

where $\pi^{\prime}$ is the principal bundle map of $\operatorname{Fr}(B, M)$ and the over line is a lift with respect to that map. It is easy to see that this is well defined for a horizontal form, since it does not depend on the choice of lift. Note also that by definition $\tau=\tau_{h}+\tau_{v}$. The following proposition is the main result of this section.

Proposition 3.17 ( $\mathrm{O}^{\prime}$ Neill on Principal Bundles). $\tau_{v}$ corresponds to $\mathrm{T}$ and $\tau_{\mathrm{h}}$ corresponds to A.

Proof. Note that $\tau$ is described by the difference of the connection on $\operatorname{Fr}(M)$ and the connection on $\operatorname{Fr}(B, M)$. The connection on $\operatorname{Fr}(M)$ gives rise to the covariant derivative $\nabla^{M}$, and the connection on $\operatorname{Fr}(B, M)$ to $\tilde{\nabla}$. As we have shown before, the connection extended from $\tilde{\nabla}$ splits into two connections which are the Levi Civita connection on the fibers and the horizontal submanifolds, if they exist. Even if they do not, a quick inspection of equation (3.38), using the matrix form of the reduced connection, shows that

$$
\tilde{\nabla}_{\xi} X=\mathcal{H} \nabla_{\xi}^{M} X
$$

if $\xi$ and $X$ are horizontal and

$$
\tilde{\nabla}_{\eta} Y=\mathcal{V} \nabla_{\eta}^{M} Y,
$$

if $\eta$ and $Y$ are vertical. The unique extension of this to $\operatorname{Fr}(M)$ gives the connection

$$
\hat{\nabla}_{\chi} \mathrm{Z}:=\mathcal{H} \nabla_{\chi}^{\mathrm{M}} \mathcal{H} \mathrm{Z}+\mathcal{v} \nabla_{\chi}^{\mathrm{M}} \mathcal{V Z}
$$

for $\chi$ an arbitrary tangent vector and $Z$ an arbitrary vector field on $M$. This can be verified by showing that the above is indeed a covariant derivative on $M$ and that it restricts to $\tilde{\nabla}$ if both $\chi$ and $Z$ are vertical, or both are horizontal. The latter is immediately clear, the former some simple calculations.

We see now, that

$$
\begin{aligned}
& \nabla_{\chi}^{\mathrm{M} Z}=\mathcal{H} \nabla_{\mathcal{H} \chi}^{\mathrm{M}} \mathcal{H} \mathrm{CZ}+\mathcal{H}_{\mathcal{V}_{\chi}}^{\mathrm{M}} \mathcal{H} \mathrm{C}+\mathcal{H}_{\mathcal{H}_{\chi}}^{\mathrm{M}} \mathcal{V Z}+\mathcal{H} \nabla_{\mathcal{V} \chi}^{M} \mathcal{M Z} \\
& +\mathcal{V} \nabla_{\mathcal{H} \chi}^{M} \mathcal{H} Z+\mathcal{V} \nabla_{\mathcal{V} \chi}^{M} \mathcal{H} Z+\mathcal{V} \nabla_{\mathcal{H}_{\chi}}^{M} \mathcal{V} Z+\mathcal{V} \nabla_{\mathcal{V}_{\chi}}^{M} \mathcal{V} Z \\
& =A_{\chi} Z+T_{\chi} Z+\hat{\nabla}_{\chi} Z \text {, }
\end{aligned}
$$


hence the difference of connections indeed gives $A+T$. Finally, notice that if $x$ is horizontal then $T$ vanishes, as does $\tau_{v}$. If on the other hand $\chi$ is vertical, then $A$ vanishes, as does $\tau_{h}$.

The principal bundle of frames $\operatorname{Fr}(B)$ of $B$ can be pulled back to $M$ via $\pi$. The Levi Civita connection $\phi^{\mathrm{B}}$ on $\operatorname{Fr}(\mathrm{B})$ can also be pulled back to a connection $\tilde{\phi}$ on $\pi^{*} \operatorname{Fr}(\mathrm{B})$ together with the structure equation

$$
\tilde{\phi}+\tilde{\theta}_{\mathrm{B}} \wedge \tilde{\phi}=0,
$$

where $\tilde{\theta}_{B}$ is the pull back of the solder form $\theta_{B}$ on $\operatorname{Fr}(B)$. If we pull this solder form into $\operatorname{Fr}(B, M)$, we get a form $\theta_{B}^{\prime}$, where the obvious restriction map is used $k: \operatorname{Fr}(B, M) \rightarrow$ $\pi^{*} \operatorname{Fr}(B)$. A calculation similar to that in remark (3.9) shows that $\theta_{B}^{\prime}$ agrees with the part of $\theta^{\prime}$, that has values in $\mathbb{R}^{b}$. If we split $\theta^{\prime}$ into two parts, $\theta_{1}$ and $\theta_{2}$ with values in $\mathbb{R}^{\mathrm{b}}$ and $\mathbb{R}^{\mathrm{m}-\mathrm{b}}$, and $\psi$ into $\psi_{1}$ and $\psi_{2}$ with values in $\mathfrak{s o}(\mathrm{b})$ and $\mathfrak{s o}(\mathrm{m}-\mathrm{b})$, then the structural equation 3.51 of $\psi$ decomposes into

$$
\begin{aligned}
& \mathrm{d} \theta_{1}+\psi_{1} \wedge \theta_{1}+\tau \wedge \theta_{2}=0 \\
& \mathrm{~d} \theta_{2}+\psi_{2} \wedge \theta_{2}+\tau \wedge \theta_{1}=0 .
\end{aligned}
$$

If we restrict the first equation to $\pi$-horizontal vectors, the last term vanishes and we see that $\psi_{1}$ is the Levi Civita connection pulled back from B. Such a restriction also turns $\tau$ into $\tau_{h}$ and we get the formula

$$
k^{*} \pi^{*} \phi^{B}+\tau_{h}=i^{*} \phi_{M},
$$

on $\operatorname{Fr}(B, M)$, if we restrict to vectors lifted from $B$. This is the recovery of $\mathrm{O}^{\prime} \mathrm{Neill}$ 's formula for the connections [O'N66, Lemma 3.4].

\subsubsection{Forms on $\operatorname{Fr}_{\mathrm{SO}}\left(\mathrm{N}, \mu^{-1}(0)\right)$}

Applying the last section to the reduction $\operatorname{Fr}_{\mathrm{SO}}\left(\mathrm{N}, \mu^{-1}(0)\right)$ of $\operatorname{Fr}_{\mathrm{SO}}\left(\mu^{-1}(0)\right)$ on $\mu^{-1}(0)$, we get the equation

$$
j_{3}^{\mathbb{R} *} \varphi^{\mu^{-1}(0)}=\psi_{1}+\psi_{2}+\tau^{\prime},
$$

where $\psi_{1}$ is the pull back of the Levi Civita connection on $\mathrm{N}$.

\subsubsection{Forms on $\operatorname{Fr}_{\mathrm{Sp}}(\mathrm{N}, \mathrm{M})$}

Now we will do a similar construction on the quaternionic side of the reduction for $\mathrm{Fr}_{S p}(\mathrm{~N}, M)$ As with $\mathrm{Fr}_{S O}\left(\mu^{-1}(0), M\right), \mathrm{Fr}_{S p}(N, M)$ will in general not be horizontal in $\iota^{*} \mathrm{Fr}_{S p}(M)$. Using Proposition 3.7 we construct a connection $\tilde{\varphi}^{\mathbb{H}}$ with the decomposition

$$
\mathfrak{s p}(m)=\mathfrak{s p}(n) \oplus \mathfrak{o}(k) \oplus \mathfrak{f},
$$


induced by an inclusion of $\mathbf{S} \mathbf{p}(n) \times \mathbf{S O}(k)$ in $\mathbf{S p}(\mathrm{m})$ as described in the beginning. As before, the obvious choice of complement will satisfy the necessary condition (3.7).

We get the projected connection form $\tilde{\varphi}^{\mathbb{H}}$ which decomposes into two equivariant one-forms $\phi_{1}^{\mathbb{H}}$ and $\phi_{2}^{\mathbb{H}}$ with values in $\mathfrak{s p}(n)$ and $\mathfrak{s o}(k)$ respectively and a difference form $\tau^{\mathbb{H}}$ with

$$
\phi_{1}^{\mathbb{H}}+\phi_{2}^{\mathbb{H}}+\tau^{\mathbb{H}}=j_{2}^{\mathbb{H} *} \hat{\varphi}^{\mathbb{H}}
$$

\subsection{Final Result}

\subsubsection{Preparation}

Let us recall the connections of the real reductions. On $\operatorname{Fr}_{\mathrm{SO}}\left(\mu^{-1}(0), M\right)$ we have equation 3.32

$$
\phi_{1}^{\mathbb{R}}+\phi_{2}^{\mathbb{R}}+\tau^{\mathbb{R}}=j_{2}^{\mathbb{R} *} \hat{\varphi}^{\mathbb{R}},
$$

where $\hat{\varphi}^{\mathbb{R}}$ is the pull back of the Levi Civita connection on $M . \phi_{1}^{\mathbb{R}}$ is the pull back of the Levi-Civita connection of $\mu^{-1}(0)$, which in turn decomposes on $\operatorname{Fr}_{\mathrm{SO}}\left(\mathrm{N}, \mu^{-1}(0)\right)$ according to equation (3.62).

The connection $\psi_{1}+\psi_{2}$ on $\operatorname{Fr}_{S O}\left(N, \mu^{-1}(0)\right)$ can be extended back to a connection $\tilde{\psi}_{1}+\tilde{\psi}_{2}$ on $\operatorname{Fr}_{\mathrm{SO}}\left(\mu^{-1}(0)\right)$, so that we have

$$
\tilde{\psi}_{1}+\tilde{\psi}_{2}+\tilde{\tau}^{\prime}=\varphi^{\mu^{-1}(0)}
$$

where $\tilde{\tau}^{\prime}$ is defined by this equation (and hence the pull back of it is $\tau^{\prime}$.) So if we pull back this equation to $\mathrm{Fr}_{\mathrm{SO}}\left(\mu^{-1}(0), M\right)$, we get

$$
k_{1}^{\mathbb{R} *} \tilde{\psi}_{1}+k_{1}^{\mathbb{R} *} \tilde{\psi}_{2}+k_{1}^{\mathbb{R} *} \tilde{\tau}^{\prime}=\phi_{1}^{\mathbb{R}},
$$

and combining this with 3.65

$$
k_{1}^{\mathbb{R} *} \tilde{\psi}_{1}+k_{1}^{\mathbb{R} *} \tilde{\psi}_{2}+k_{1}^{\mathbb{R} *} \tilde{\tau}^{\prime}+\phi_{2}^{\mathbb{R}}+\tau^{\mathbb{R}}=j_{2}^{\mathbb{R} *} \hat{\varphi}^{\mathbb{R}} .
$$

Since $i_{3}^{*} \hat{\varphi}^{\mathbb{R}}=\hat{\varphi}^{\mathbb{H}}$, we can identify the right hand side of the equation above and of 3.64) if we pull back by $i_{3}$,

$$
i_{3}^{*}\left(k_{1}^{\mathbb{R} *} \tilde{\psi}_{1}+k_{1}^{\mathbb{R} *} \tilde{\psi}_{2}+k_{1}^{\mathbb{R} *} \tilde{\tau}^{\prime}+\phi_{2}^{\mathbb{R}}+\tau^{\mathbb{R}}\right)=\phi_{1}^{\mathbb{H}}+\phi_{2}^{\mathbb{H}}+\tau^{\mathbb{H}} .
$$

To understand which terms correspond, it is a good idea to visualize where the different forms take their values. If we identify $\mathbb{H}^{n}$ with $\mathbb{R}^{4 n}$ such that $a+i b+j c+k d$ gets mapped to $(a, b, c, d)\left(a, b, c, d \in \mathbb{R}^{n}\right)$, we identify $n \times n$ quaternionic matrices $A+i B+j C+k D$ with $4 n \times 4 n$ real matrices of the form 


$$
\left(\begin{array}{cccc}
A & -B & -C & -D \\
B & A & -D & C \\
C & D & A & -B \\
D & -C & B & A
\end{array}\right)
$$

If we use a frame $p \in \operatorname{Fr}_{S p}(N, M)$ to identify $\iota^{*}(T M)$ with $\mathbb{R}^{4 m}$, we see that both sides of the equations take values in matrices of the form

$$
\left(\begin{array}{cc}
M_{1} & -M_{2}^{t} \\
M_{2} & M_{3}
\end{array}\right),
$$

where $M_{1}$ is a $4 n \times 4 n, M_{2}$ a $4 k \times 4 n$ and $M_{3}$ a $4 k \times 4 k$ block matrix of the type given above. Using the quaternionic splitting, we can decompose the $M_{i}$ into $A_{i}, B_{i}, C_{i}$ and $D_{i}$. Note that in $M_{3}$ only $A_{3}$ (the diagonal) is non vanishing, because of the inclusion $\mathrm{SO}(\mathrm{k}) \hookrightarrow \mathrm{Sp}(\mathrm{k}), A \mapsto A+i A+j A+k A$.

The components of the matrices $M_{i}$ are of course only defined up to the choice of frame $p \in \operatorname{Fr}_{S p}(N, M)$. However, two different frames differ by a matrix in $S p(n) \times$ $\mathrm{SO}(\mathrm{k})$, which leaves the components of $M_{3}$ and the component-rows of $M_{2}$ invariant. $M_{1}$ and the columns of $M_{2}$ get transformed by conjugation with a $\mathbb{H}$-linear matrix.

Define the matrix $M_{2}^{1}$ to be the first $k$ rows of $M_{2}, M_{2}^{2}$ to be the other $3 k$ rows and $M_{3}^{1}$ as $A_{3},\left(M_{3}^{2}\right)^{t}=\left(B_{3}, C_{3}, D_{3}\right)$ and $M_{3}^{3}$ as the matrix $M_{3}$ without the first $k$ columns and first $k$ rows. Hence we may write 3.71 as

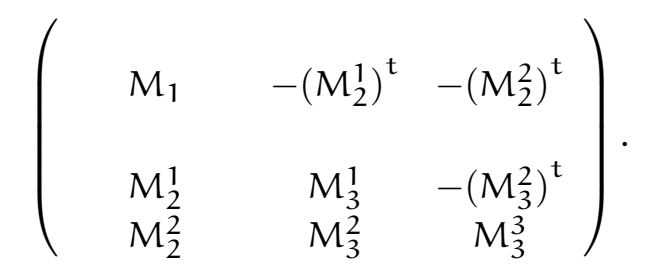

Starting with the right hand side of the equation $3.69, \phi_{1}^{\mathbb{H}}$ takes values $M_{1}, \phi_{2}^{\mathbb{H}}$ in $M_{3}$ and $\tau^{\mathbb{H}}$ the remaining $M_{2}$ matrix. On the left hand side, $\tilde{\psi}_{1}$ takes values in the $M_{1}, \tilde{\psi}_{2}$ in $M_{3}^{1}, \tilde{\tau}^{\prime}$ in $M_{2}^{1}, \phi_{2}^{\mathbb{R}}$ in $M_{3}^{3}$ and $\tau^{\mathbb{R}}$ in the remaining $M_{2}^{2}$ and $M_{3}^{2}$ matrices.

\subsubsection{The Results}

The equations (3.69) and the following analysis of the previous section allows us to recover some of the results from [GN92]. First we see that

$$
i_{3}^{*} k_{1}^{\mathbb{R} *} \tilde{\psi}_{1}=\phi_{1}^{\mathbb{H}} \quad \Rightarrow \quad k^{*} \psi_{1}=\phi_{1}^{\mathbb{H}},
$$

because both sides take values in $M_{1}$. If we pull back the Levi-Civita connection on $\operatorname{Fr}_{S O}(N)$ to $\operatorname{Fr}_{S p}(N, M)$ via $\operatorname{Fr}_{S p}(N)$, we get $\phi_{1}^{\mathbb{H}}$ because of this equation. Hence the 
pull back to $\operatorname{Fr}_{S p}(\mathrm{~N})$ takes values in $\mathrm{H}$-linear matrices, in other words the connection reduces to one on $\mathrm{Fr}_{S p}(\mathrm{~N})$. This shows that $\mathrm{N}$ is indeed a hyperkähler manifold.

A more constructive argument can be given by noting that the Levi-Civita connection on $M$ is $G$-invariant, for the canonical choice of extension of the $G$ action to $\mathrm{Fr}_{\mathrm{SO}}(\mathrm{M})$. This remains true for $\phi_{1}^{\mathbb{H}}$ and a careful examination shows that it can be pushed down to $\operatorname{Fr}_{\mathrm{Sp}}(\mathrm{N})$.

If we continue with $M_{3}$, we see that for $\xi \in \mathfrak{g}, \mathrm{II}(\cdot, \xi)$, which is described by $M_{3}^{2}=0$, vanishes.

The fact that $M_{3}$ is only non-vanishing on the diagonal, gives a connection between the covariant derivative on the fibers of $\pi: \mu^{-1}(0) \rightarrow N$, and the normal derivative of $\mu^{-1}(0)$ described by $\phi_{2}^{\mathbb{R}}$, i.e. $D_{\xi} Y:=\operatorname{pr}_{T^{-1}(0)^{\perp}} \nabla_{\xi}^{M} Y$, for $\xi \in T \mu^{-1}(0)$ and $Y \in$ $\Gamma\left(\mu^{-1}(0), T \mu^{-1}(0)^{\perp}\right)$ (see e.g. [KN69, p. VII]). Precisely, we have for all $A \in\{I, J, K\}$

$$
\nabla_{\xi}^{\mathrm{F}} X=\mathrm{d} \mu^{\mathrm{A}} \circ \mathrm{D}_{\xi}\left(A K^{\eta}\right), \quad \forall \xi, \eta \in \mathfrak{g},
$$

where $\nabla^{\mathrm{F}}$ is the connection on the fiber.

Let us now focus on $M_{2}$. From proposition 3.15 we know that $M_{2}^{2}$ and $M_{3}^{2}$ give the second fundamental form and from proposition 3.17) we know that $M_{2}^{1}$ is $A+T$, the O'Neill tensors. Hence

$$
M_{2}(\xi)=p^{-1} \circ\left(\begin{array}{cccc}
\left(A_{\xi}+T_{\xi}\right)(\cdot) & \left(A_{\xi}+T_{\xi}\right)(\mathrm{I} \cdot) & \left(A_{\xi}+\mathrm{T}_{\xi}\right)(\mathrm{J} \cdot) & \left(\mathrm{A}_{\xi}+\mathrm{T}_{\xi}\right)(\mathrm{K} \cdot) \\
\mathrm{II}^{\mathrm{I}}(\xi, \cdot) & \mathrm{II}^{\mathrm{I}}(\xi, \mathrm{I} \cdot) & \mathrm{II}^{\mathrm{I}}(\xi, \mathrm{J} \cdot) & \mathrm{II}^{\mathrm{I}}(\xi, \mathrm{K} \cdot) \\
\mathrm{II}^{\mathrm{J}}(\xi, \cdot) & \mathrm{II}^{\mathrm{J}}(\xi, \mathrm{I} \cdot) & \mathrm{II}^{\mathrm{J}}(\xi, \mathrm{J} \cdot) & \mathrm{II}^{\mathrm{J}}(\xi, \mathrm{K} \cdot) \\
\mathrm{II}^{\mathrm{K}}(\xi, \cdot) & \mathrm{II}^{\mathrm{K}}(\xi, \mathrm{I} \cdot) & \mathrm{II}^{\mathrm{K}}(\xi, \mathrm{J} \cdot) & \mathrm{II}^{\mathrm{K}}(\xi, \mathrm{K} \cdot)
\end{array}\right) \circ p,
$$

where $\mathrm{II}^{A}$ is the second fundamental form of $\mu^{-1}(0) \hookrightarrow M$ projected onto $A \mathfrak{g} \subset$ $\mathrm{T \mu}^{-1}(0)^{\perp}$ and $p \in \mathrm{Fr}_{\mathrm{Sp}}(\mathrm{N}, \mathrm{M})$ is a frame (restricted in a suitable way). Using the form 3.70 of the matrix, we get the following results (recall the notation $\iota^{*}(\mathrm{TM})=$ $\left.\mathrm{H} \oplus \mathfrak{g} \otimes_{\mathbb{R}} \mathbb{H}\right)$.

If $\xi \in H$ and $\cdot \in H$, then the first row of $M_{2}$ becomes $-\frac{1}{2} R(\xi, \cdot), \ldots$, where $R$ is the curvature of $\mu^{-1}(0) \rightarrow N$ as discussed before. This yields that for all $\xi, \eta \in H$,

$$
-\frac{1}{2} \mathrm{R}(\xi, \eta)=\mathrm{II}^{\mathrm{I}}(\xi, \mathrm{I} \eta)=\mathrm{II}^{\mathrm{J}}(\xi, \mathrm{J \eta})=\mathrm{II}^{\mathrm{K}}(\xi, \mathrm{K} \eta) .
$$

Here $\mathrm{II}^{\mathrm{I}}=\mathrm{d} \mu^{\mathrm{I}} \circ$ II. Note that this in particular implies that $R$ is hyperholomorphic, i.e. of type $(1,1)$ with respect to all complex structures (on $N$, viewing $R$ as a two form on N).

If $\xi \in \mathfrak{g}$ and $\cdot \in \mathrm{H}$, then the first row becomes $T_{\xi} \cdot=\mathcal{V} \nabla_{\xi}^{\mu^{-1}(0)} \cdot, \ldots$, where $\nabla^{\mu^{-1}(0)}$ is the Levi-Civita connection on $\mu^{-1}(0)$ and $\nu$ is the vertical projection in $T \mu^{-1}(0)$ from $\pi: \mu^{-1}(0) \rightarrow \mathrm{N}$. This can be described as the negative of the Weingarten map $\mathcal{W}_{\xi}(\cdot)$ of the fibers of $\pi$. Hence we get for all $\xi \in \mathfrak{g}, \eta \in H$,

$$
-\mathcal{W}_{\xi}(\eta)=\mathrm{II}^{\mathrm{I}}(\xi, \mathrm{I} \eta)=\mathrm{II}^{\mathrm{J}}(\xi, \mathrm{J \eta})=\mathrm{II}^{\mathrm{K}}(\xi, \mathrm{K} \eta) .
$$


However, since II is symmetric, $\mathrm{II}(\xi, \cdot)=0$, hence the Weingarten map of the fibers vanish, in other words, the fibers are totally geodesic.

If $\xi \in \mathrm{H}$ and $\cdot \in \mathfrak{g}$, the discussion needs to be carried out in $-M_{2}^{t}$. Using the formula for $A$ and $T$ (and that $\mathrm{II}(\xi, \cdot)=0$ ), we see that

$$
\operatorname{pr}_{\mathrm{H}} \circ \nabla_{\xi}^{\mu^{-1}(0)} \mathrm{X}=0,
$$

for all $\xi \in \mathrm{H}$ and $X \in \Gamma\left(\mu^{-1}(0), \mathfrak{g}\right)$, which is already clear from $\mathcal{W}_{X}(\xi)=0$. Both $\xi$ and in $\mathfrak{g}$ again yield that the second fundamental forms of the fibers of $\pi$ vanish. 


\section{Chapter 4}

\section{Gauge Theories}

All of the theories mentioned in this chapters are vast fields in mathematics. This chapter only tries to give the absolute minimum of definitions needed to follow the next chapters.

\section{1 (Anti) Self-Duality Equations}

Definition 4.1. Let $M$ be a 4-manifold with $\mathbf{S O}(4)$-holonomy (i.e. Riemannian and oriented), $\mathrm{P} \stackrel{\mathrm{G}}{\rightarrow} M$ a principal $\mathrm{G}$ bundle on $M$ and let $A \in \mathcal{C}(\mathrm{P})$ be a connection. $A$ is called an anti self-dual connection, if

$$
\pi_{+}\left(\mathrm{F}^{\mathrm{A}}\right)=0,
$$

and a self-dual instanton if $\pi_{-}\left(F^{A}\right)=0$, where $\pi_{ \pm}$are the (orthogonal) projections onto

$$
\Omega^{2}(M, \operatorname{Ad}(P)) \cong \Omega_{+}^{2}(M, \operatorname{Ad}(P)) \oplus \Omega_{-}^{2}(M, \operatorname{Ad}(P)) .
$$

Remark 4.2. Since

$$
\pi_{+}=\frac{1}{2}(\mathbb{1}+\star) \quad \text { and } \quad \pi_{-}=\frac{1}{2}(\mathbb{1}-\star)
$$

a connection $A$ is anti self-dual if and only if $F^{A}+\star F^{A}=0$, and self-dual if and only if $\mathrm{F}^{\mathrm{A}}-\star \mathrm{F}^{\mathrm{A}}=0$.

\subsection{Spin (7)-Instantons}

Spin(7)-instantons are a close relative of instantons on 4-manifolds, as can bee seen by the next definition.

Definition 4.3. Let $M$ be a 8-manifold with $\operatorname{Spin}(7)$-holonomy, $P \stackrel{G}{\rightarrow} M$ a principal $G$ bundle on $M$ and let $A \in \mathcal{C}(P)$ be a connection. $A$ is called a $\operatorname{Spin}(7)$-instanton if

$$
\pi_{7}\left(F^{A}\right)=0 \text {, }
$$


where $\pi_{7}$ is the (orthogonal) projection onto

$$
\Omega^{2}(M, \operatorname{Ad}(P)) \cong \Omega_{7}^{2}(M, \operatorname{Ad}(P)) \oplus \Omega_{21}^{2}(M, \operatorname{Ad}(P)) .
$$

Remark 4.4. Since

$$
\pi_{7}=\frac{1}{2}(\mathbb{1}+\mathrm{G}(\Theta))
$$

a connection $A$ is a Spin(7)-instanton if and only if

$$
\mathrm{F}^{\mathrm{A}}+\star\left(\Theta \wedge \mathrm{F}^{\mathrm{A}}\right)=0
$$

\subsection{Generalized Seiberg-Witten Theory}

Generalized Seiberg-Witten Theory is a generalization of Seiberg-Witten theory to allow for targets other than $\mathbb{H}$. The fundamental idea is to replace the fibers of the spinbundles with a more complicated hyperkähler manifold M. Detailed analysis shows that the hyperkähler manifold has to have a trihamiltonian action by a group $G$ and a rotating action. This allows to define a Seiberg-Witten theory with target $M$. For brevity we will not explain any details of this theory unless they are needed for the following. The interested reader is referred to [Pido4; Cal1o; Hay15a].

The first simplification is that we are only looking at rotating actions that factor through $\mathbf{S O}(3)$. As such we can define the involved principal bundles as a direct product. Furthermore, we won't explain how to define the non-linear generalized Dirac operator $\mathscr{D}_{\mathfrak{a}}$, but will only give an equivalent criteria to $\mathscr{D}_{\mathrm{a}} \mathfrak{u}=0$ for a spinor $u$ and a connection $a$.

Definition 4.5. Let $X$ be an oriented Riemannian 4-manifold and let $Q \rightarrow X$ be a principal $G$ bundle. Let $P_{+} \rightarrow X$ be the principal SO(3)-bundle of frames in $\wedge_{+}^{2} X$ and fix the Levi-Civita connection on it. Assume further that $M$ is a hyperkähler manifold which has a trihamiltonian action by $\mathrm{G}$ with moment map $\mu: M \rightarrow \operatorname{Im} \mathbb{H} \otimes \mathfrak{g}$ and a rotating action which factors through $\mathbf{S U}(2) \rightarrow \mathbf{S O}(3)$. The generalized Seiberg-Witten equations for a pair $(a, u) \in \mathcal{C}(Q) \times \mathfrak{C}^{\infty}\left(P_{+} \times{ }_{x} Q, M\right)^{G \times S O}(3)$ are given by

$$
\mathscr{D}_{\mathrm{a}} \mathrm{u}=0, \quad \mu \circ \mathrm{u}=\mathrm{F}_{+}^{\mathrm{a}} \text {. }
$$

Remark 4.6. As mentioned we will only give an equivalent criteria for the first equation, without defining $\mathscr{D}_{a} u$. For the second equation notice that $\mu \circ \mathfrak{u}: \mathrm{Q} \rightarrow \operatorname{Im} \mathbb{H} \otimes \mathfrak{g}$ is $\mathrm{G} \times \mathbf{S O}(3)$-equivariant and as such gives a section of the associated vector bundle $\Lambda_{+}^{2} X^{\vee} \otimes \operatorname{Ad}(Q)=\Omega_{+}^{2}(X, A d(Q))$.

For the first equation, notice that $a \in \mathcal{C}(\mathrm{Q})$ together with the Levi-Civita connection on $\mathrm{P}_{+}$defines a connection $\hat{\mathrm{a}}$ on $\mathrm{P}_{+} \times_{X} \mathrm{Q} \rightarrow \mathrm{X}$. Interpreting this connection as a horizontal subspace, we can restrict $D u: T_{(p, q)}\left(P_{+} \times_{x} Q\right) \rightarrow T_{u(p, q)} M$ to this subspace. Horizontal lifting identifies this space with $T_{\pi(p, q)} X$ at the corresponding point $x=$ 
$\pi(p, q)$. The base point $p \in P_{+}$defines a quaternionic structure on $T_{x} M$, by defining any base of $T_{x} M$ that induces $p$ on $\Lambda_{+}^{2} T_{x} M$ as quaternionic. Now

$$
D_{x} u: T_{x} M \rightarrow T_{u(p, q)} M
$$

is a linear map between two quaternionic vector spaces and we define $\mathscr{D}_{\mathrm{a}} u=0$ if and only if $D_{x} u$ has no quaternionic linear part for all $x \in X$. Such a map is called aholomorphic.

Remark 4.7. Notice that a linear map f between quaternionic vector spaces $\left(\mathrm{V}, \mathrm{I}_{1}, \mathrm{I}_{2}, \mathrm{I}_{3}\right)$ and $\left(\mathrm{W}, \mathrm{J}_{1}, \mathrm{~J}_{2}, \mathrm{~J}_{3}\right)$ has no quaternionic part if and only if the projection onto the quaternionic linear part vanishes. This projection is given by some multiple of $f \mapsto(f-$ $\left.\sum_{i=1}^{3} I_{i} f J_{i}\right)$. Since this map itself is quaternionic linear, it suffices to check this vanishing for a quaternionic base; in our case it suffices to check this on a single vector $\xi \in T_{x} M$. Using a quaternionic base $e_{0}, \ldots, e_{3}$ of $T_{x} M$, the condition becomes

$$
\operatorname{Du}\left(\tilde{e}_{0}\right)-\sum_{i=1}^{3} I_{i} \operatorname{Du}\left(\tilde{e}_{i}\right)=0,
$$

where : denotes the horizontal lift. Finally, to horizontally lift a vector, we can also lift it arbitrarily and remove the vertical part, i.e.

$$
\tilde{\xi}=\bar{\xi}-\mathrm{K}^{\hat{\mathrm{a}}(\bar{\xi})},
$$

where $\hat{a}$ is the connection on $\mathrm{P}_{+} \times_{X} \mathrm{Q}$ and $\bar{\xi}$, some lift of $\xi$. Using that $u$ is $\mathbf{S O}(3) \times \mathrm{G}$ equivariant (technically anti-equivariant) we get

$$
\mathrm{D}_{x} \mathrm{u}(\xi)=\left(\mathrm{Du}\left(\bar{\xi}-\mathrm{K}_{\mathrm{P}_{+} \times \mathrm{x}}^{\hat{\mathrm{a}}(\bar{\xi})}\right)\right)_{\mathbb{H}}=\left(\mathrm{Du}(\bar{\xi})+\mathrm{K}_{\mathrm{M}}^{\hat{\mathrm{a}}(\bar{\xi})}\right)_{\mathbb{H}^{\prime}}
$$

where $(\cdot)_{\mathbb{H}}$ denotes the quaternionic projection.

Remark 4.8. Note finally, that if $M$ is $\mathbb{H}^{n}$ or an affine space over $\mathbb{H}^{n}$, then there are two fundamental $\mathbb{H}$ structures on $M$, one by left multiplication of $i, j, k \in \mathbb{H}$ and one by right multiplication by $(-i,-j,-k)$. We will call the first a left-left structure (if $X$ was a quaternionic vector space, then the first left indicates that the multiplication on $X$ would be from the left), and the second a left-right structure. Decomposing $\mathrm{Du}=\mathrm{Du_{0 }}+i \mathrm{D} u_{1}+j \mathrm{Du} u_{2}+k D u_{3}$ we arrive at the signs

$$
\begin{gathered}
\text { left-left structure: }\left\{\begin{array}{l}
\mathrm{D} u_{0}\left(e_{0}\right)+\mathrm{D} u_{1}\left(e_{1}\right)+\mathrm{D} u_{2}\left(e_{2}\right)+\mathrm{D} u_{3}\left(e_{3}\right) \\
\mathrm{D} u_{1}\left(e_{0}\right)-\mathrm{D} u_{0}\left(e_{1}\right)-D u_{3}\left(e_{2}\right)+D u_{2}\left(e_{3}\right) \\
D u_{2}\left(e_{0}\right)+D u_{3}\left(e_{1}\right)-D u_{0}\left(e_{2}\right)-D u_{1}\left(e_{3}\right) \\
D u_{3}\left(e_{0}\right)-D u_{2}\left(e_{1}\right)+D u_{1}\left(e_{2}\right)-D u_{0}\left(e_{3}\right)
\end{array}\right. \\
\text { left-right structure: }\left\{\begin{array}{l}
D u_{0}\left(e_{0}\right)-D u_{1}\left(e_{1}\right)-D u_{2}\left(e_{2}\right)-D u_{3}\left(e_{3}\right) \\
D u_{1}\left(e_{0}\right)+D u_{0}\left(e_{1}\right)-D u_{3}\left(e_{2}\right)+D u_{2}\left(e_{3}\right) \\
D u_{2}\left(e_{0}\right)+D u_{3}\left(e_{1}\right)+D u_{0}\left(e_{2}\right)-D u_{1}\left(e_{3}\right) \\
D u_{3}\left(e_{0}\right)-D u_{2}\left(e_{1}\right)+D u_{1}\left(e_{2}\right)+D u_{0}\left(e_{3}\right)
\end{array}\right.
\end{gathered}
$$

for the projection 4.10 . 


\section{Chapter 5}

\section{Dimensional Reduction}

\subsection{Dimensional Reduction of a Gauge Theory}

\subsubsection{Motivation}

There are many different concepts of reductions in Gauge Theory. The one we are considering here is the following. Assume we have a gauge theory on a base manifold $X$, with $\operatorname{target} M$. Now if $G$ is a Lie group which acts on $X$, such that $X / G$ is again a smooth manifold, then we want to reduce the gauge theory $X \rightarrow M$ to one $X / G \rightarrow M$. In this section we will discuss this construction in detail. The next section shows how this can be used to show that Haydys-Witten equations are invariant Spin(7)instantons.

\subsubsection{Formulation}

Throughout this section, let $X$ be a smooth manifold, $G$ a Lie group and $\pi: P \rightarrow X$ a principal G-bundle.

Definition 5.1 (Reductions). Let $\mathrm{H}$ be a Lie group.

- We call $(\mathrm{X}, \mathrm{H})$ a reducing pair if $\mathrm{H}$ acts on the right of $\mathrm{X}, \mathrm{X} / \mathrm{H}$ is a smooth manifold and $\mathrm{X} \rightarrow \mathrm{X} / \mathrm{H}$ is a principal $\mathrm{H}$-bundle.

- Let $(\mathrm{X}, \mathrm{H})$ be a reducing pair. If the action of $\mathrm{H}$ lifts to a right action on $\mathrm{P}$ by bundle automorphisms, such that $\mathrm{P} / \mathrm{H}$ is a smooth manifold and $\mathrm{P} \rightarrow \mathrm{P} / \mathrm{H}$ is a principal $\mathrm{H}$-bundle, then we call $(\mathrm{X}, \mathrm{P}, \mathrm{H})$ a reducing triple.

Remark 5.2 (Reducing Triples and Pull Backs). A reducing triple $(\mathrm{X}, \mathrm{P}, \mathrm{H})$ is given by a pullback diagram

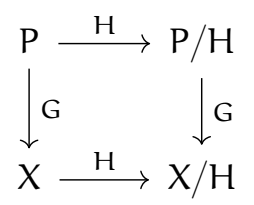


of principal bundles.

Notation 5.3 (Maps, Connections and Horizontal Forms of a Reducing Triple). Given a reducing triple $(\mathrm{X}, \mathrm{P}, \mathrm{H})$, we denote the projections of the various principal bundles by

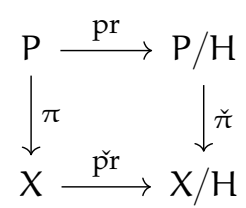

The connections on $\mathrm{X} \rightarrow \mathrm{X} / \mathrm{H}$ and $\mathrm{P} / \mathrm{H} \rightarrow \mathrm{X} / \mathrm{H}$ are denoted by $\mathrm{C}(\mathrm{X})$ and $\mathrm{C}(\mathrm{P} / \mathrm{H})$ respectively. Since "connections on $\mathrm{P}$ " is ambiguous, we will index those with the projection map, so connections on $\mathrm{P} \rightarrow \mathrm{X}$ are denoted by $\mathcal{C}_{\pi}(\mathrm{P})$ and connections on $\mathrm{P} \rightarrow \mathrm{P} / \mathrm{H}$ by $\mathcal{C}_{\mathrm{pr}}(\mathrm{P})$.

If we talk about a horizontal form $\omega$ on $X$ this means that $\mathfrak{l}_{\mathfrak{h}} \omega=0$. We denote those by $\Omega_{\mathfrak{h}}(\mathrm{X})$ and similarly for $\mathrm{P} / \mathrm{H}$. On $\mathrm{P}$ we have to distinguish between $\mathfrak{g}$ and $\mathfrak{h}$ horizontal, we denote those by $\Omega_{\mathfrak{g}}$ and $\Omega_{\mathfrak{h}}$ respectively.

Remark 5.4 (Reducing Connections). Given a reducing triple (X, P, H), we are interested in the $\mathrm{H}$-invariant connections. Since the $\mathrm{G}$ and $\mathrm{H}$ actions on $\mathrm{P}$ commute, $\mathrm{R}_{\mathrm{h}}$ is a $\mathrm{G}$ equivariant map for every $h \in H$, hence for every $\xi \in \mathfrak{g}, K_{G}^{\xi}$ is $R_{h}$ related. This implies that that the induced action

$$
\mathrm{TP} \curvearrowleft \mathrm{H}, \quad \mathrm{h} \mapsto \mathrm{DR}_{\mathrm{h}},
$$

leaves the subbundle $\mathfrak{g} \subset$ TP invariant in a way so that it induces an action $\mathfrak{g} \curvearrowleft \mathrm{H}$ which is trivial.

Definition 5.5 (Invariant Connection). Let $(\mathrm{X}, \mathrm{P}, \mathrm{H})$ be a reducing triple. Define an action

$$
\Omega^{1}(\mathrm{P}, \mathfrak{g}) \curvearrowleft \mathrm{H},
$$

via the trivial action $\mathfrak{g} \curvearrowleft \mathrm{H}$ and the induced action $\Omega(\mathrm{P}) \curvearrowleft \mathrm{H}$ by pullback.

$\mathrm{H}$-invariant connections $\mathrm{e}_{\pi}^{\mathrm{H}}(\mathrm{P}) \subset \mathcal{C}_{\pi}(\mathrm{P}) \subset \Omega^{1}(\mathrm{P}, \mathfrak{g})^{\mathrm{G}}$ are connections invariant under this action.

Remark 5.6. In later chapters, we sometimes are interested in describing gauge theories in such a situation, even if they are not $\mathrm{H}$-invariant. This is also often called reduction in the literature (sometimes compactification in physics). To avoid using the term reduction again, we will call this description of a gauge theory in differential equation form. The examples will show why this could be considered a fitting name.

Example 5.7 (Invariant Connections on $\mathbb{R}^{2}$ ). H-invariant connections $\mathrm{C}_{\pi}^{\mathrm{H}}(\mathrm{P})$ can be obtained by pullback of connections $\mathrm{C}(\mathrm{P} / \mathrm{H})$. Not all invariant connections are of this type though. 
Consider for example $X=\mathbb{R}^{2}, H=\mathbb{R}$ acting by translation on the second factor

$$
R_{h}(x, y)=(x, y+h) \quad(x, y) \in X, h \in H,
$$

and $\mathrm{P}=\underline{\mathrm{S}}^{1}$. Then $(\mathrm{X}, \mathrm{P}, \mathrm{H})$ is a reducing triple with the canonical lift of $\mathrm{H}$ to $\mathrm{P}$. Since $\mathrm{X} / \mathrm{H}=\mathbb{R}$ and $\mathrm{P} / \mathrm{H}=\underline{\mathrm{S}}^{1} \rightarrow \mathbb{R}$, connections $\mathrm{C}(\mathrm{P} / \mathrm{H})$ are one forms $i \Omega^{1}(\mathbb{R})$ with values in $\mathfrak{u}(1) \cong i \mathbb{R}$.

Hence pulled back connections from $\mathrm{C}(\mathrm{P} / \mathrm{H})$ are all of the form

$$
A=\mathfrak{i}(f \circ \check{\mathrm{pr}}) \mathrm{dx} \quad f \in \mathrm{C}^{\infty}(X / H), \breve{\mathrm{pr}}: X \rightarrow X / H .
$$

On the other hand, if we identify $\mathcal{C}_{\pi}(\mathrm{P}) \cong i \Omega^{1}(\mathrm{X}), \mathrm{H}$-invariant connections are given by

$$
B=\mathfrak{i}(f \circ \check{p r}) d x+g d y, \quad f \in C^{\infty}(X / H), g \in C^{\infty}(X)^{H},
$$

so that $\hat{p r}^{*} \mathrm{C}(\mathrm{P} / \mathrm{H}) \subset \mathcal{C}_{\pi}^{\mathrm{H}}(\mathrm{P})$ is an honest subspace.

A natural question is what the quotient (as affine spaces) of invariant connections by pulled back connections is. The next proposition investigates this question. Note that in the example above, the two spaces differed by a smooth function $\mathfrak{C}^{\infty}(\mathrm{X} / \mathrm{H})=$ $\mathfrak{C}^{\infty}(\mathbb{R})$.

Proposition 5.8 (SES of affine spaces for a reducing triple). Let $(\mathrm{X}, \mathrm{P}, \mathrm{H})$ be a reducing triple. Then there is a canonical short exact sequence of affine spaces

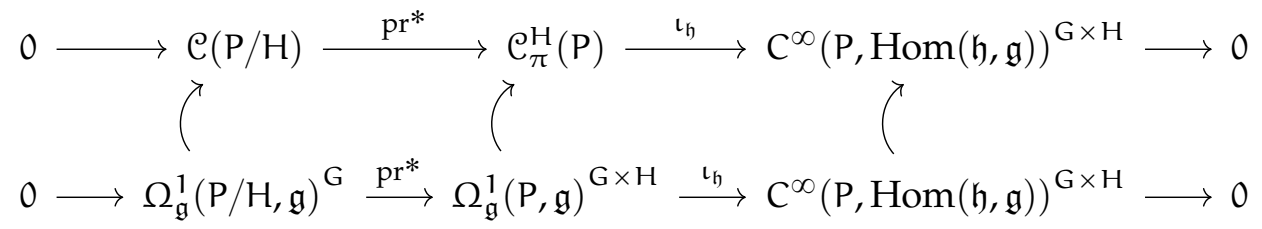

Here the transitive and free action of the G-equivariant and horizontal one forms on the spaces of connections is the usual (by addition) and $\mathrm{C}^{\infty}(\mathrm{P}, \operatorname{Hom}(\mathfrak{h}, \mathfrak{g}))^{\mathrm{G} \times \mathrm{H}}$ is considered an affine space over itself.

For completeness, let us recall the lef 1 actions on the various spaces. If $\omega$ is in either of $\Omega_{\mathfrak{g}}^{1}(\mathrm{P} / \mathrm{H}, \mathfrak{g})^{\mathrm{G}}, \mathrm{C}(\mathrm{P} / \mathrm{H})$ or $\mathrm{C}_{\pi}^{\mathrm{H}}(\mathrm{P})$, then the action of $\mathrm{g} \in \mathrm{G}$ is given by

$$
\text { g.w }=\operatorname{Ad}_{g} R_{g}^{*} \omega .
$$

For $\omega \in \Omega_{\mathfrak{g}}^{1}(\mathrm{P}, \mathfrak{g})^{\mathrm{G} \times \mathrm{H}}$, the action of $(\mathrm{g}, \mathrm{h}) \in \mathrm{G} \times \mathrm{H}$ is given by

$$
(g, h) \cdot \omega=\operatorname{Ad}_{g} R_{g}^{*} R_{h}^{*} \omega,
$$

and on $\varphi \in \mathrm{C}^{\infty}(\mathrm{P}, \operatorname{Hom}(\mathfrak{h}, \mathfrak{g}))^{\mathrm{G} \times \mathrm{H}}$ the action of $(\mathrm{g}, \mathrm{h}) \in \mathrm{G} \times \mathrm{H}$ is given by

$$
(g, h) \cdot \varphi=\operatorname{Ad}_{g} \circ\left(R_{g}^{*} R_{h}^{*} \varphi\right) \circ \operatorname{Ad}_{h^{-1}} .
$$

${ }^{1}$ See remark 5.10 
Remark 5.9 (SES of affine spaces). Exact sequences of affine spaces are defined through exact sequences of the underlying vector subspaces.

Remark 5.10 (Left Actions and Right Actions). It is important to keep track of right and left actions, unfortunately this can be cumbersome at times. Take for example a G-equivariant $k$-form $\omega \in \Omega^{k}(P, \mathfrak{g})^{G}$. The right action $P \curvearrowleft G$ induces a right action on $\Lambda^{k} \mathrm{TP}$ and a left action on $\Lambda^{\mathrm{k}} \mathrm{TP}^{\vee}$. The left action $\mathrm{G} \curvearrowright \mathfrak{g}$ by Ad induces a right action via $g \mapsto \operatorname{Ad}_{g^{-1}}$. So $\omega: \wedge^{k} T P \rightarrow \mathfrak{g}$, is G-equivariant with respect to these right actions. If we view $\omega \in\left(\Omega^{k}(P) \otimes \mathfrak{g}\right)^{G}$, then it is invariant under the left action $D R_{g}^{\vee} \otimes A_{g}$ and $\left(D R_{g}^{\vee} \otimes A d_{g}\right) \omega=A d_{g} R_{g}^{*} \omega$.

Proof. Let us first focus on the lower row of $(5.8)$. We show that the maps are welldefined. The first map pr* is just the usual identification of forms on the base manifold and $\mathrm{H}$-invariant forms on the bundle. Since pr is also G-equivariant (this is how we defined the $\mathrm{G}$-action on $\mathrm{P} / \mathrm{H})$, the pullback of a $\mathrm{G}$-invariant form is still G-invariant.

If $\omega \in \Omega_{\mathfrak{g}}^{1}(\mathrm{P}, \mathfrak{g})^{\mathrm{G} \times \mathrm{H}}$, we define $\varphi=\mathfrak{l}_{\mathfrak{h}} \omega$. It is obviously a smooth function on $\mathrm{P}$ with values in $\mathfrak{h}^{\vee} \otimes \mathfrak{g}=\operatorname{Hom}(\mathfrak{h}, \mathfrak{g})$, we only need to check the invariance. To show that note that the fundamental vector fields $K_{H}$ are H-equivariant

$$
\mathrm{K}_{\mathrm{H}}^{\mathrm{Ad}_{h^{-1}} \xi}=\mathrm{DR}_{\mathrm{h}} \mathrm{K}_{\mathrm{H}}^{\xi} \quad \mathrm{h} \in \mathrm{H}, \xi \in \mathfrak{h},
$$

and since $\mathrm{G}$ and $\mathrm{H}$ commute, every diffeomorphism $\mathrm{R}_{\mathrm{g}}$ is $\mathrm{H}$-equivariant, which implies that $K_{H}^{\xi}$ is $R_{g}$ related for every $\xi \in \mathfrak{h}$ and $g \in G$,

$$
K_{H}^{\xi} \circ R_{g}=D R_{g} \circ K_{H}^{\xi} \quad h \in H, \xi \in \mathfrak{h} .
$$

Now for $(g, h) \in G \times H, p \in P$ and $\xi \in \mathfrak{h}$

$$
\begin{aligned}
& \left(R_{g}^{*} R_{h}^{*} \varphi\right)_{p} \operatorname{Ad}_{h^{-1}}(\xi)=\varphi_{p g h} \operatorname{Ad}_{h^{-1}}(\xi)=\omega_{p g h}\left(\left.K_{H}^{A d_{h-1} \xi}\right|_{p g h}\right) \\
& \stackrel{5.12}{-} \omega_{\mathrm{pgh}}\left(\left.\left.D R_{h}\right|_{\mathrm{pg}} \mathrm{K}_{\mathrm{H}}^{\xi}\right|_{\mathrm{pg}}\right)=\left(D R_{\mathrm{h}}^{*} \omega\right)_{\mathrm{pg}}\left(\left.\mathrm{K}_{\mathrm{H}}^{\xi}\right|_{\mathrm{pg}}\right) \\
& \stackrel{5.10}{=} \omega_{p g}\left(\left.\mathrm{~K}_{\mathrm{H}}^{\xi}\right|_{\mathrm{pg}}\right) \stackrel{5.13}{=} \omega_{\mathrm{pg}}\left(\left.\left.\mathrm{DR}_{\mathrm{g}}\right|_{\mathrm{p}} \mathrm{K}_{\mathrm{H}}^{\xi}\right|_{\mathrm{p}}\right) \\
& \stackrel{5.10}{=} \operatorname{Ad}_{g^{-1}} \omega_{p}\left(\left.K_{\mathrm{H}}^{\xi}\right|_{p}\right)=\operatorname{Ad}_{g^{-1}} \varphi_{p}(\xi) \text {, }
\end{aligned}
$$

so that $\operatorname{Ad}_{g}\left(R_{g}^{*} R_{h}^{*} \varphi\right)_{p} \operatorname{Ad}_{h^{-1}}(\xi)=\varphi_{p}(\xi)$ as claimed.

The injectivity of $\mathrm{pr}^{*}$ follows immediately from the usual identification of forms on the base and horizontal H-invariant forms on the total space, because pr* restricted to its image

$$
\operatorname{im}\left(\mathrm{pr}^{*}\right)=\Omega_{\mathfrak{g}, \mathfrak{h}}^{1}(\mathrm{P}, \mathfrak{g})^{\mathrm{G} \times \mathrm{H}}
$$

is invertible. This image also implies that $\operatorname{im}(\operatorname{pr})=\operatorname{ker}\left(\mathfrak{t}_{\mathfrak{h}}\right)$ which leaves us to show that $\iota_{\mathfrak{h}}$ is surjective. We will show this in 5.12

The upper row in (5.8) follows by similar arguments. The well-definedness follows by exactly the same calculation and the injectivity of the lower row's pr* implies the 
injectivity upstairs. The image of pr* are simply $\mathfrak{h}$-horizontal connections which shows the exactness in the middle and the surjectivity of $\mathfrak{l}_{\mathfrak{h}}$ will be shown in 5.14

Example 5.11 (Transitive action). Before we work out the general case, let us look at an example where we have a transitive action $X \curvearrowleft H$, so that $X / H=\{*\}$. In this case a connection

$$
\mathrm{TP} \cong \mathfrak{g} \oplus \mathfrak{h} \rightarrow \mathfrak{g}
$$

is determined by the map $\mathfrak{h} \rightarrow \mathfrak{g} \in \mathfrak{C}^{\infty}(P, \operatorname{Hom}(\mathfrak{h}, \mathfrak{g}))^{\mathrm{G} \times H} \cong \operatorname{Hom}(\mathfrak{h}, \mathfrak{g})$. This agrees with the statement, since any pulled back connection is the trivial connection (because $\left.\Omega^{1}(\{*\})=0\right)$. A closer look shows that we have a splitting of the short exact sequence, because there is a unique choice in connection $X \rightarrow X / H$.

Proposition 5.12 (Splitting of Lower Row). In the situation of Proposition $\sqrt{5.8}$, if we fix a connection $\mathrm{B} \in \mathcal{C}(\mathrm{X})$, then the lower row of (5.8) splits.

In particular this implies that $\mathfrak{l}_{\mathfrak{h}}$ on the lower row is surjective.

If $\mathrm{X} \rightarrow \mathrm{X} / \mathrm{H}$ is a Riemannian submersion, then there is a canonical choice for $\mathrm{B}$ given by the orthogonal complement of the vertical subspaces of TX.

Proof. We use the following commuting diagram,

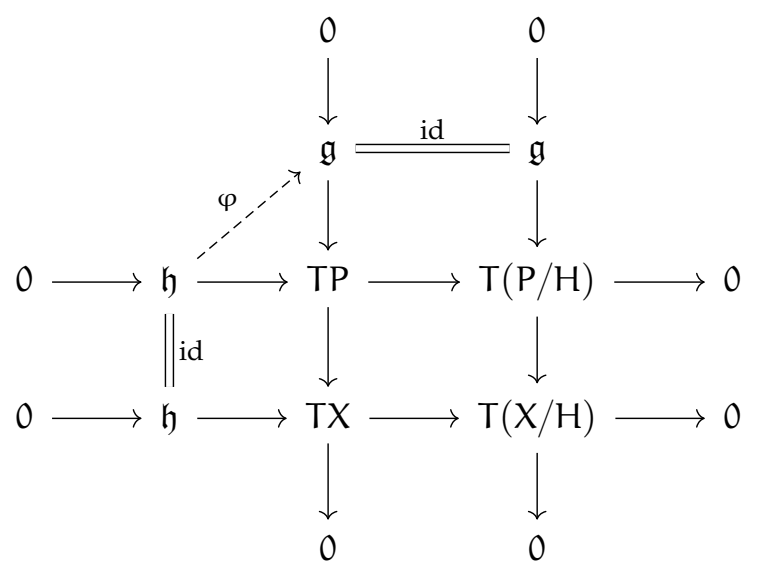

of vector bundles on P (we omitted the pull back symbols on some of the bundles), where any row and column is a short exact sequence of H-equivariant, respectively G-equivariant, vector bundles.

Now $B \in \mathcal{C}(X)$ induces via pull back a $G$-invariant connection in $\mathcal{C}_{\mathrm{pr}}^{\mathrm{G}}(\mathrm{P})$, which gives a G-invariant and H-equivariant splitting of the top row. This defines

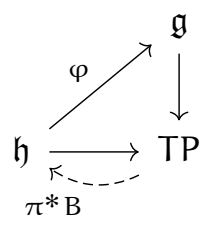

in the above diagram. We will show that 
1. $\varphi \circ \pi^{*} \mathrm{~B} \in \Omega_{\mathfrak{g}}^{1}(\mathrm{P}, \mathfrak{g})^{\mathrm{G} \times \mathrm{H}}$ and

2. $\mathfrak{l}_{\mathfrak{h}}\left(\varphi \circ \pi^{*} \mathrm{~B}\right)=\varphi \in \mathrm{C}^{\infty}(\mathrm{P}, \operatorname{Hom}(\mathfrak{h}, \mathfrak{g}))^{\mathrm{G} \times \mathrm{H}}$,

which will show the claim.

Ad 1. We calculate

$$
\begin{aligned}
R_{g}^{*} R_{h}^{*}\left(\varphi \circ \pi^{*} B\right) & =\left(R_{g}^{*} R_{h}^{*} \varphi\right) \circ\left(R_{g}^{*} R_{h}^{*} \pi^{*} B\right) \\
& =\operatorname{Ad}_{g^{-1}} \circ \varphi \circ \operatorname{Ad}_{h} \circ A_{h^{-1}} \circ \pi^{*} B \\
& =A d_{g^{-1}} \circ \varphi \circ \pi^{*} B
\end{aligned}
$$

which is the required behavior under the action. Note that it is important here that $\mathrm{B}$ is G-invariant.

Ad 2. This follows immediately from the fact that $\pi^{*} B$ is a projection onto $\mathfrak{h} \subset \mathrm{TP}$, so $\mathfrak{\iota}_{\mathfrak{h}} \pi^{*} \mathrm{~B}=\mathrm{id}_{\mathfrak{h}}$.

Notation 5.13. To simplify the notation, we will also denote the pull back of $B\left(\pi^{*} B\right)$ with $B$ in the following.

Proposition 5.14 (Splitting of Upper Row). If we fix connections $\mathrm{B} \in \mathrm{C}(\mathrm{X})$ and $\mathrm{C} \in \mathrm{C}(\mathrm{P} / \mathrm{H})$ the upper row of proposition (5.8) splits.

In particular this implies that $\mathfrak{l}_{\mathfrak{h}}$ on the upper row is surjective.

Proof. This follows immediately from Proposition (5.12) and the fact that the top row is affine over the bottom row, i.e. $C \in \mathcal{C}(P / H)$ identifies $\mathcal{C}(P / H) \cong \Omega_{\mathfrak{g}}^{1}(P / H, \mathfrak{g})^{G}$ and its pull back identifies $\mathcal{C}_{\pi}^{\mathrm{H}}(\mathrm{P}) \cong \Omega_{\mathfrak{g}}^{1}(\mathrm{P}, \mathfrak{g})^{\mathrm{G} \times \mathrm{H}}$

Theorem 5.15 (The Curvature of the Reduction). Let $(\mathrm{X}, \mathrm{P}, \mathrm{H})$ be a reducing triple, $\mathrm{B} \in$ $\mathcal{C}(\mathrm{X})$ and $\mathrm{C} \in \mathrm{C}(\mathrm{P} / \mathrm{H})$ and with respect to their splitting let

$$
A=(a, \varphi)=\operatorname{pr}^{*} a+\varphi \circ B \in \mathcal{C}(P / H) \oplus C^{\infty}(P, \operatorname{Hom}(\mathfrak{h}, \mathfrak{g}))^{G \times H} .
$$

Then the curvature of A satisfies the following identity

$$
\mathrm{F}^{\mathrm{A}}=\operatorname{pr}^{*} \mathrm{~F}^{\mathrm{a}}+\mathrm{d}_{\mathrm{a}} \varphi \stackrel{\circ}{\wedge} \mathrm{B}+\varphi \circ \mathrm{dB}+\frac{1}{2}[\varphi \circ \mathrm{B} \wedge \varphi \circ \mathrm{B}],
$$

Proof. If $A$ is a connection and $\omega$ an equivariant and horizontal 1-form, then the curvature of $A+\omega$ is given by

$$
F^{A+\omega}=F^{A}+d_{A} \omega+\frac{1}{2}[\omega \wedge \omega] .
$$


which follows immediately from the defining equation $F^{A}=d A+\frac{1}{2}[A \wedge A]$. Using this for $\omega=\mathrm{B} \circ \varphi$, we get

$$
\begin{aligned}
\mathrm{F}^{\mathrm{A}} & =\operatorname{pr}^{*} \mathrm{~F}^{\mathrm{a}}+\mathrm{d}_{\mathrm{a}}(\varphi \circ \mathrm{B})+\frac{1}{2}[\varphi \circ \mathrm{B} \wedge \varphi \circ \mathrm{B}] \\
& =\operatorname{pr}^{*} \mathrm{~F}^{\mathrm{a}}+\mathrm{d}_{\mathrm{a}} \varphi \stackrel{\wedge}{\wedge} \mathrm{B}+\varphi \circ \mathrm{dB}+\frac{1}{2}[\varphi \circ \mathrm{B} \wedge \varphi \circ \mathrm{B}],
\end{aligned}
$$

where we used that $d_{a} B=d B$, because the $G$ representation of $\mathfrak{h}$ is trivial (compare to Remark 5.4).

Remark 5.16. Note that this equation holds not only on $P$, but also on $X$, where $\mathrm{F}^{\mathrm{A}}, \mathrm{pr}^{*} \mathrm{~F}^{\mathrm{a}} \in \Omega^{2}(\mathrm{X}, \operatorname{Ad}(\mathrm{P}))$ and $\varphi \in \Gamma(\operatorname{Hom}(\underline{\mathfrak{h}}, \operatorname{Ad}(\mathrm{P})))$ (recall that $\mathrm{B}$ is also pulled back from $X)$.

Corollary 5.17. If in the setting of theorem 5.15) $\varphi \in \Omega^{1}(\mathrm{P}, \operatorname{Hom}(\mathfrak{h}, \mathfrak{g}))^{\mathrm{G} \times \mathrm{H}}$ is a Lie algebra homomorphism, then

$$
\mathrm{F}^{\mathrm{A}}=\operatorname{pr}^{*} \mathrm{~F}^{\mathrm{a}}+\mathrm{d}_{\mathrm{a}} \varphi \stackrel{\circ}{\wedge} \mathrm{B}+\varphi \circ \mathrm{F}^{\mathrm{B}} .
$$

Remark 5.18. In the setting of theorem (5.15) the summands of 5.23, live on different subspaces of the tangent space. Indeed, let us split $T X=\xi \oplus \mathfrak{h}$, where $\xi$ is the complementary distribution of $\mathfrak{h} \subset \mathrm{TX}$ defined by B. Then

$$
\Lambda^{2} \mathrm{TX}=\Lambda^{2} \xi \oplus \xi \otimes \mathfrak{h} \oplus \Lambda^{2} \mathfrak{h},
$$

and we can now associate the terms to the different subspaces,

$$
\mathrm{F}^{\mathrm{A}}=\underbrace{\operatorname{pr}^{*} \mathrm{~F}^{\mathrm{a}}}_{\wedge^{2} \xi}+\underbrace{\mathrm{d}_{\mathrm{a}} \varphi \stackrel{\circ}{\wedge} \mathrm{B}}_{\xi \otimes \mathfrak{h}}+\varphi \circ \mathrm{dB}+\underbrace{\frac{1}{2}[\varphi \circ \mathrm{B} \wedge \varphi \circ \mathrm{B}]}_{\wedge^{2} \mathfrak{h}} .
$$

The term $\varphi \circ \mathrm{dB}$ is problematic since a priori it does not belong to any one of these spaces. To combat this in the examples we will assume that we have a trivial bundle $X \rightarrow X / H$, which allows us to simply use the Maurer-Cartan form of the Lie Group $H$ as the connection $B$. But then $d B=-\frac{1}{2}[B \wedge B]$, so $\varphi \circ d B \in \Lambda^{2} \mathfrak{h}$.

\subsection{Examples}

Let us explore how to work with these equations by applying them to some wellknown examples, i.e. reductions of the anti self-duality equations. After that we will use these to reduce $\mathbf{S p i n}(7)$-instantons to $\mathbf{G}_{\mathbf{2}}$-monopoles and the Haydys-Witten equations. The former is mentioned in $\left[\overline{D S}{ }_{11 b}\right]$, whereas the latter is a new result. As mentioned in Remark 5.16, we may interpret these equations on $P$ and $X$. If the bundle $X \rightarrow X / H$ is trivial, we may even interpret the equation on $X / H$. To simplify the notation, we will simply write $\mathrm{F}^{\mathrm{a}}$ instead of $\mathrm{pr}^{*} \mathrm{~F}^{\mathrm{a}}$, no matter where we consider the equations. 


\subsubsection{Bogomolny Equations}

Let $\mathrm{G}$ be some Lie group and $(\mathrm{H} \times \mathrm{X}, \mathrm{P}, \mathrm{H})$ a reducing triple consisting of a oriented Riemannian 3-dimensional manifold $\mathrm{X}$, a principal G-bundle $\mathrm{P} \rightarrow \mathrm{H} \times \mathrm{X}$ and a 1dimensional abelian Lie Group $\mathrm{H}$ acting on itself, and by isometries on $\mathrm{H} \times \mathrm{X}$. Use the Maurer Cartan form of $\mathrm{H}$ as the connection B. We reduce the anti self-duality equations (see Definition 4.1) on $\mathrm{H} \times \mathrm{X}$

$$
\star_{4} \mathrm{~F}^{\mathrm{A}}+\mathrm{F}^{\mathrm{A}}=0 .
$$

By the construction, $A \in \mathcal{C}(P)$ splits into a connection $a \in \mathcal{C}(P / H)$ and a Higgs field $\phi \in$ $\Gamma(X, \operatorname{Hom}(\mathfrak{h}, \operatorname{Ad}(\mathfrak{g})))$ (for notational consistency, we denote the Higgs field by $\phi$ instead of $\varphi$ ). Here we have used that $\operatorname{Ad}(\mathfrak{h})$ is trivial, because $H$ is abelian. Since $\mathfrak{h}$ is also one-dimensional, we get $\phi \in \Gamma(\mathrm{X} / \mathrm{H}, \mathrm{Ad}(\mathfrak{g})$ ) (the orientation makes this identification unique). Using the formula for the curvature, we get

$$
F^{A}=F^{a}+d_{a} \phi \wedge B
$$

since $\mathrm{dB}=0$ by the Maurer Cartan formula and $\Lambda^{2} \mathfrak{h}=0$. The fact that the underlying manifold is given by $\mathrm{H} \times \mathrm{X}$, we can decompose the equation

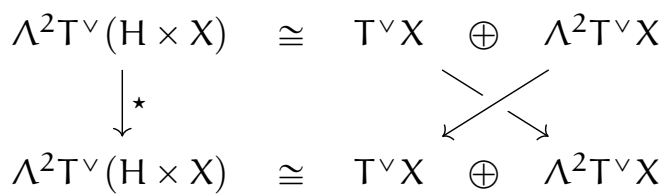

Using Lemma 2.9 we see that the anti self-duality equations give two equations,

$$
\mathrm{B} \wedge\left(\star_{3}\left(\mathrm{~F}^{\mathrm{a}}\right)-\mathrm{d}_{\mathrm{a}} \phi\right)=0
$$

and

$$
-\star_{3} d_{a} \phi+F^{a}=0 .
$$

One quickly realizes that these are the same equation, which on $X$ is given by

$$
F^{\mathrm{a}}=\star_{3} \mathrm{~d}_{\mathrm{a}} \phi
$$

These are the so called Bogomolny equations on X.

\subsubsection{Hitchin System}

Similar to the last example, pick the reducing triple $(H \times X, P, H)$, where $H$ is a two dimensional abelian Lie group, and $X$ is an (oriented) 2-dimensional manifold. 


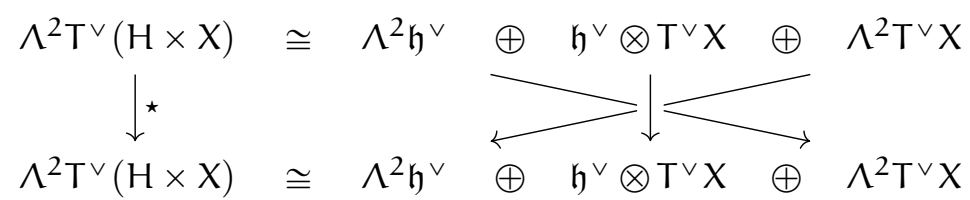

This results in three equations

$$
\begin{aligned}
& \star \mathrm{F}^{\mathrm{a}}+\frac{1}{2}[\varphi \circ \mathrm{B} \wedge \varphi \circ \mathrm{B}]=0, \\
& \mathrm{~F}^{\mathrm{a}}+\star \frac{1}{2}[\varphi \circ \mathrm{B} \wedge \varphi \circ \mathrm{B}]=0, \\
& \star \mathrm{d}_{\mathrm{a}} \varphi \wedge \mathrm{B}+\mathrm{d}_{\mathrm{a}} \varphi \wedge \mathrm{B}=0,
\end{aligned}
$$

of which the first two are the same. With the identification

$$
\Phi=\varphi_{1}+i \varphi_{2}
$$

the first becomes $F=\frac{i}{2}\left[\Phi, \Phi^{*}\right]$, and the last $D_{\bar{z}} \Phi=0$. These are the so called Hitchin Equations on $\mathrm{X}$.

\subsubsection{Nahm Equations}

Similar to the last example, pick the reducing triple $(H \times X, P, H)$, where $H$ is a three dimensional abelian Lie group, and $X$ is an (oriented) one-dimensional Riemannian manifold. Then a connection $A \in \mathcal{C}(P)$ induces a connection $a \in \mathcal{C}(P / H)$ and a Higgs field. Picking a basis of $\mathfrak{h}$ (that is oriented and orthonormal with respect to the structure of $\mathrm{H} \times \mathrm{X}$ ) we identify $\mathfrak{h} \cong \mathbb{R}^{3}$ and decompose

$$
\varphi=\left(\varphi_{1}, \varphi_{2}, \varphi_{3}\right) \in \Gamma(X, \operatorname{Hom}(\mathfrak{h}, \operatorname{Ad}(P))) \cong \mathbb{R}^{3 \vee} \otimes \Gamma(X, \operatorname{Ad}(P)) .
$$

Let $\mathrm{dh}^{1}, \mathrm{dh}^{2}$ and $\mathrm{dh}^{3}$ denote the left invariant one forms induced by the choice of basis above, and $t$ denote the coordinate of $X$ with respect to some chart. Using for the connection $B$ the Maurer Cartan form (so that $d B=0$ ), the curvature yields

$$
\begin{aligned}
\mathrm{F}^{\mathrm{A}} & =\mathrm{d}_{\mathrm{a}} \varphi \wedge \mathrm{B}+\frac{1}{2}[\varphi \circ \mathrm{B} \wedge \varphi \circ \mathrm{B}] \\
& =\sum_{j=1}^{3} \nabla^{\mathrm{a}} \varphi_{j} \mathrm{dt} \wedge \mathrm{dh}^{j}+\frac{1}{2} \sum_{i, j=1}^{3}\left[\varphi_{i}, \varphi_{j}\right] \mathrm{dh}^{i} \wedge d h^{j} \\
& =d t \wedge\left(\sum_{j=1}^{3} \nabla^{\mathrm{a}} \varphi_{j} \mathrm{dh}^{j}\right)+\sum_{1 \leqslant i<j \leqslant 3}\left[\varphi_{i}, \varphi_{j}\right] d h^{i} \wedge d h^{j}
\end{aligned}
$$


where we have used that $\Lambda^{2} \xi=0$, i.e. there is no curvature on a 1-dimensional manifold.

The anti self duality equations then give two sets of identical equations, given by

$$
\begin{aligned}
\nabla^{\mathrm{a}} \varphi_{1}+\left[\varphi_{2}, \varphi_{3}\right] & =0 \\
-\nabla^{\mathrm{a}} \varphi_{2}+\left[\varphi_{1}, \varphi_{3}\right] & =0 \\
\nabla^{\mathrm{a}} \varphi_{3}+\left[\varphi_{1}, \varphi_{2}\right] & =0
\end{aligned}
$$

on $X$. These are the Nahm equations.

\subsubsection{Rotationally Invariant Nahm Equations}

Similar to the last example, we may write $\mathbb{R}^{4} \backslash\{0\} \cong \mathbb{R}^{>0} \times \mathbf{S U}(2)$ and take the (left) action of $\mathbf{S U}(2)$ on itself to reduce the $\mathbf{S U}(2)$ invariant anti self duality equations to $\mathbb{R}^{>0}$. This time $a \in \mathcal{C}(\mathrm{P} / \mathrm{SU}(2))$ and

$$
\varphi \in \Gamma\left(\mathbb{R}^{>0}, \operatorname{Hom}(\underline{\mathfrak{s u}(2)}, \underline{\mathfrak{g}})\right) \cong \mathfrak{s u}(2)^{\vee} \otimes \mathfrak{C}^{\infty}\left(\mathbb{R}^{>0}, \mathfrak{g}\right) .
$$

Using the Pauli matrices as a basis for $\mathfrak{s u}(2)$, we can decompose $\varphi$ in components $\varphi_{1}, \varphi_{2}, \varphi_{3} \in \mathfrak{C}^{\infty}\left(\mathbb{R}^{<0}, \mathfrak{g}\right)$. For $\mathrm{B}$ we take the trivial connection given by the Maurer Cartan form of $\mathbf{S U}(2)$,

$$
B=\sum_{j=1}^{3} \sigma^{j} \otimes d \sigma^{j},
$$

where $\sigma^{j}$ are the Pauli matrices and $d \sigma^{j}$ are the left invariant one forms dual to the left invariant vector fields defined by the Pauli matrices. The formula for the curvature now gives

$$
\begin{aligned}
\mathrm{F}^{\mathrm{A}} & =\mathrm{d}_{\mathrm{a}} \varphi \stackrel{\circ}{\wedge} \mathrm{B}+\varphi \circ \mathrm{dB}+\frac{1}{2}[\varphi \circ \mathrm{B} \wedge \varphi \circ \mathrm{B}] \\
& =\sum_{j=1}^{3} \nabla^{\mathrm{a}} \varphi_{j} \mathrm{~d} x^{1} \wedge \mathrm{d} \sigma^{j}+\sum_{i<j}\left(2 \varepsilon_{i j k} \varphi_{k}+\left[\varphi_{i}, \varphi_{j}\right]\right) d \sigma^{i} \wedge d \sigma^{j}
\end{aligned}
$$

where we used the Maurer Cartan equation for the expression dB. A similar calculation as for the ordinary Nahm equations give

$$
\begin{aligned}
\nabla^{\mathrm{a}} \varphi_{1}+2 \varphi_{1}+\left[\varphi_{2}, \varphi_{3}\right] & =0 \\
-\nabla^{\mathrm{a}} \varphi_{2}-2 \varphi_{2}+\left[\varphi_{1}, \varphi_{3}\right] & =0 \\
\nabla^{\mathrm{a}} \varphi_{3}+2 \varphi_{3}+\left[\varphi_{1}, \varphi_{2}\right] & =0 .
\end{aligned}
$$

These are the rotational (or perturbed) version of the Nahm Equations. 


\subsubsection{ADHM Equations}

In this example we consider a reducing triple $(\mathrm{H}, \mathrm{P}, \mathrm{H})$, where $\mathrm{H}$ acts on itself and is an oriented Riemannian Lie Group. Then the equations dictate

$$
\mathrm{F}^{\mathrm{A}}=\frac{1}{2}[\varphi \circ \mathrm{B}, \varphi \circ \mathrm{B}]
$$

Note in particular, that

$$
\varphi \in \Gamma\left(\{p \mathrm{t}\}, \operatorname{Hom}\left(\mathbb{R}^{4}, \mathfrak{g}\right)\right) \cong \mathbb{R}^{4 \vee} \otimes \mathfrak{g},
$$

that is, $\varphi$ can be identified with four elements in $\mathfrak{g}$, given by four matrices if $G$ is a matrix Lie Group. The equation for the curvature now implies

$$
0=\star F^{A}+F^{A}=\sum_{1 \leqslant i<j \leqslant 4}\left[\varphi_{i}, \varphi_{j}\right] \star\left(d h_{i} \wedge d h_{j}\right)+\sum_{1 \leqslant i<j \leqslant 4}\left[\varphi_{i}, \varphi_{j}\right] d h_{i} \wedge d h_{j}
$$

which is equivalent to

$$
\begin{array}{ll}
0=\left[\varphi_{1}, \varphi_{2}\right]+\left[\varphi_{3}, \varphi_{4}\right] & 0=\left[\varphi_{3}, \varphi_{4}\right]+\left[\varphi_{1}, \varphi_{2}\right] \\
0=\left[\varphi_{1}, \varphi_{3}\right]-\left[\varphi_{2}, \varphi_{4}\right] & 0=\left[\varphi_{2}, \varphi_{4}\right]-\left[\varphi_{1}, \varphi_{3}\right] \\
0=\left[\varphi_{1}, \varphi_{4}\right]+\left[\varphi_{2}, \varphi_{3}\right] & 0=\left[\varphi_{2}, \varphi_{3}\right]+\left[\varphi_{1}, \varphi_{4}\right]
\end{array}
$$

where we can see that every equation is given twice, so that we may reduce this to the system

$$
\begin{aligned}
& 0=\left[\varphi_{1}, \varphi_{2}\right]+\left[\varphi_{3}, \varphi_{4}\right] \\
& 0=\left[\varphi_{1}, \varphi_{3}\right]-\left[\varphi_{2}, \varphi_{4}\right] \\
& 0=\left[\varphi_{1}, \varphi_{4}\right]+\left[\varphi_{2}, \varphi_{3}\right]
\end{aligned}
$$

which are the so called ADHM Equations.

Remark 5.19. This system is often given on $\mathbb{R}^{4}$, where additional boundary conditions guarantee that it can be extended to $\mathrm{S}^{4}$.

\subsection{6 $\mathrm{G}_{2}$ Monopoles}

Let us reduce the Spin(7)-instantons by one dimension. Recall the Spin(7) instanton equation (Definition 4.3)

$$
\star\left(\Theta \wedge F^{A}\right)=-F^{A} .
$$

Let $(\mathrm{H} \times \mathrm{X}, \mathrm{P}, \mathrm{H})$ be a reducing triple consisting of a Spin(7)-manifold $\mathrm{H} \times \mathrm{X}$ of dimension 8 , a principal G-bundle $\mathrm{P} \rightarrow \mathrm{H} \times \mathrm{X}$ and a one-dimensional abelian Lie 
Group $\mathrm{H}$ acting on itself, and by isometries on $\mathrm{H} \times \mathrm{X}$. Again let $\mathrm{B}$ be the Maurer Cartan form of $\mathrm{H}$. Recall that by Corollary 2.64, $\mathrm{X}$ is a $\mathrm{G}_{\mathbf{2}}$-manifold and

$$
\Theta=\operatorname{dh} \wedge \varphi+\psi,
$$

where $h$ is the coordinate of $H$, describes the connection of the canonical forms. By splitting the bundle $\wedge^{2} T^{\vee}(H \times X)$ and analyzing the map

$$
\star(\Theta \wedge-)=\star(\underbrace{\operatorname{dh} \wedge \varphi \wedge}_{\alpha_{0}})+\star(\underbrace{\psi \wedge-}_{\beta_{0}})
$$

the following diagram arises (where we use $\operatorname{dim} H=1$ ),

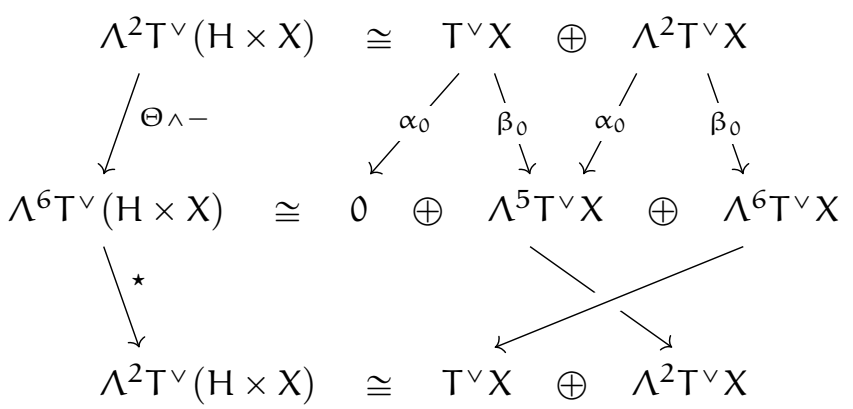

This decomposition agrees with the splitting into $\xi \otimes \mathfrak{h} \oplus \wedge^{2} \xi$ in $(5.28$. Using the formula for the curvature, we get

$$
F^{A}=\underbrace{F^{a}}_{\in \Lambda^{2} T^{\vee} X}+\underbrace{d_{a} \phi \wedge B}_{\in T^{\vee} X}
$$

where we identify $\phi \in \Gamma(\mathrm{X}, \operatorname{Ad}(\mathrm{P}))$, just like in the case of Bogomolny Monopoles. Using the observations above, we see that the Spin(7)-instanton equation decomposes into two sets of equations, given by

$$
-\mathrm{d}_{\mathrm{a}} \phi \wedge \mathrm{dh}=\star \beta_{0}\left(\mathrm{~F}^{\mathrm{A}}\right)=\star\left(\psi \wedge \mathrm{F}^{\mathrm{a}}\right)
$$

and

$$
-F^{a}=\star \alpha_{0}\left(F^{a}\right)+\star \beta_{0}\left(d_{a} \phi \wedge d h\right)=\star\left(d h \wedge \varphi \wedge F^{a}\right)+\star\left(\psi \wedge d_{a} \phi \wedge d h\right) .
$$

The former equation is equivalently given by

$$
F^{A} \wedge \psi=\star_{7} d_{a} \phi,
$$

which is the $\mathbf{G}_{\mathbf{2}}$-monopole equation. The second equation is also equivalent to this equation. To see this notice that by Lemma 2.9

$$
\star\left(d h \wedge \varphi \wedge F^{a}\right)+\star\left(\psi \wedge d_{a} \phi \wedge d h\right)=\star_{7}\left(\varphi \wedge F^{a}\right)-\star_{7}\left(\psi \wedge d_{a} \phi\right) .
$$


We decompose

$$
\Lambda^{2} T^{\vee} X \cong \Lambda_{7}^{2} \oplus \Lambda_{14}^{2}
$$

by the $\mathbf{G}_{2}$-structure (compare Proposition 2.56), and denote the projections by $\mathrm{F}^{\mathrm{a}}=$ $\mathrm{F}_{7}^{\mathrm{a}}+\mathrm{F}_{14}^{\mathrm{a}}$. Then we know that

$$
\star_{7}\left(\varphi \wedge \mathrm{F}^{\mathrm{a}}\right)=2 \mathrm{~F}_{7}^{\mathrm{a}}-\mathrm{F}_{14}^{\mathrm{a}},
$$

by the eigenspace decomposition and hence

$$
\mathrm{F}^{\mathrm{a}}+\star\left(\varphi \wedge \mathrm{F}^{\mathrm{a}}\right)=3 \mathrm{~F}_{7}^{\mathrm{a}}
$$

So the equation is equivalent to

$$
\mathrm{G}(\psi)\left(\mathrm{d}_{\mathrm{a}} \phi\right)=3 \mathrm{~F}_{7}^{\mathrm{a}},
$$

which in turn is equivalent to $\mathrm{G}(\psi)^{2}\left(\mathrm{~F}^{\mathrm{a}}\right)=3 \mathrm{~F}_{7}^{\mathrm{a}}$. To see that the latter holds, notice that $G(\psi)^{2}: \Lambda^{2} \rightarrow \Lambda^{2}$ is a $\mathbf{G}_{2}$-map. By Schur's lemma it will be a multiple on all irreducible components. Because wedging with $\psi$ maps into $\Lambda^{6}$, which is 7-dimensional and irreducible, the $\Lambda_{14}^{2}$ components gets mapped to 0 . Now applying $G\left(\psi^{2}\right)$ to any element in $\Lambda_{7}^{2}$ shows the result.

\subsubsection{Haydys-Witten Equations}

For the Haydys-Witten equations we proceed similar as for the $\mathbf{G}_{\mathbf{2}}$-monopoles. There are two different cases. If we have an action by $\mathbb{R}^{3}$, then we arrive at the HaydysWitten equations on a manifold with hypo structure. If we have an action by $\mathbf{S O}(3)$ then we arrive at the (perturbed) Haydys-Witten equations on a manifold with $\mathbf{S O}(4)$ structure. We will do the calculation for a $\mathbf{S O}(3)$ action, the $\mathbb{R}^{3}$ action can be derived from this.

Since the Higgs field for the Haydys-Witten equations is called $B \stackrel{\text { loc }}{=} \sum_{i=1}^{3} \varphi_{i} \sigma_{i} \in$ $\Omega_{+}^{2}(\operatorname{Ad}(P))$, we will denote the connection by $\tilde{B}$ is this secion.

Let $(\mathrm{X} \times \mathrm{H}, \mathrm{P}, \mathrm{H})$ be a reducing triple consisting of a 8-dimensional Spin(7)-manifold $X$, a principal G-bundle $P \rightarrow X$ and the three-dimensional Lie Group SO(3) acting on $X$ and $\mathrm{P}$ by isometries. Again let $\tilde{\mathrm{B}}$ be the Maurer Cartan form of $\mathrm{H}$. Recall that by Corollary 2.64. $\mathrm{X}$ is a $\mathbf{S O}(4)$-manifold and splitting the bundle $\Lambda^{2} \mathrm{~T}^{\vee}(\mathrm{H} \times \mathrm{X})$ and analyzing the map

$$
\star(\Theta \wedge-)=\star(\underbrace{\eta \wedge \varphi \wedge-}_{\alpha_{0}})+\star(\underbrace{\psi \wedge-}_{\beta_{0}})
$$

the following picture arises. Starting from the Spin(7)-instantons, we decompose the 
forms in a local frame

$$
\begin{aligned}
\varphi= & \mathrm{dh}^{123}-\mathrm{dh}^{1} \wedge \omega_{1}-\mathrm{dh}^{2} \wedge \omega_{2}-\mathrm{dh}^{3} \wedge \omega_{3}, \\
\psi= & \mathrm{d} x^{5678}-\mathrm{dh}^{23} \wedge \omega_{1}-\mathrm{dh}^{31} \wedge \omega_{2}-\mathrm{dh}^{12} \wedge \omega_{3} \\
\Theta= & \eta \wedge \varphi+\psi \\
= & \underbrace{\eta \wedge \mathrm{dh}^{123}}_{\alpha_{3}}+\underbrace{\left(-\mathrm{dh}^{12} \wedge \omega_{3}-\mathrm{dh}^{31} \wedge \omega_{2}-\mathrm{dh}^{23} \wedge \omega_{1}\right)}_{\alpha_{2}} \\
& +\underbrace{(-\eta) \wedge\left(\mathrm{dh}^{1} \wedge \omega_{1}+\mathrm{dh}^{2} \wedge \omega_{2}+\mathrm{dh}^{3} \wedge \omega_{3}\right)}_{\alpha_{1}}+\underbrace{\mathrm{d} x^{5678}}_{\alpha_{0}} .
\end{aligned}
$$

The conundrum here is that the quotient by $\mathrm{H}$ has to be chosen the way to satisfy the conditions outlined in Remark 2.66. For the case of an action of $\mathbb{R}^{3}$, we can split up the action by reducing step by step (reducing by a copy of $\mathbb{R}$ in every step) splitting of the expected directions. The calculations remain true but are more cumbersome.

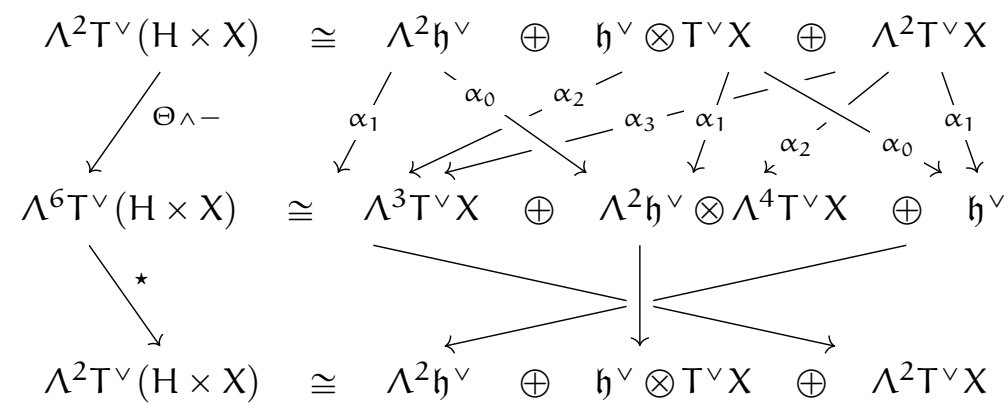

Let us study the maps a little closer.

$$
\begin{aligned}
& \Lambda^{1} T^{\vee} X \cong \mathbb{R} \oplus S^{11} \\
& \Lambda^{2} T^{\vee} X \cong S^{20} \oplus S^{02} \oplus S^{11} \\
& \Lambda^{3} T^{\vee} X \cong S^{20} \oplus S^{02} \oplus S^{11} \\
& \Lambda^{4} T^{\vee} X \cong \mathbb{R} \oplus S^{11} \\
& \mathfrak{h}^{\vee} \otimes T^{\vee} X \cong \mathfrak{h}^{\vee} \oplus \mathfrak{h}^{\vee} \otimes S^{11} \\
& \Lambda^{2} \mathfrak{h}^{\vee} \otimes \Lambda^{4} T^{\vee} X \cong \Lambda^{2} \mathfrak{h}^{\vee} \oplus \Lambda^{2} \mathfrak{h}^{\vee} \otimes S^{11} . \\
& \mathfrak{h}^{\vee} \otimes T^{\vee} X \cong S^{20} \oplus S^{20} \otimes S^{11} \cong S^{20} \oplus S^{31} \oplus S^{11} \\
& \Lambda^{2} \mathfrak{h}^{\vee} \otimes \Lambda^{4} T^{\vee} X \cong S^{20} \oplus S^{20} \otimes S^{11} \cong S^{20} \oplus S^{31} \oplus S^{11} .
\end{aligned}
$$


Now note that $\mathfrak{h}^{\vee} \cong S^{20}$, so that $\Lambda^{2} \mathfrak{h}^{\vee} \cong S^{20}$. We identify

$$
\Lambda_{+}^{2} \cong \mathfrak{h}^{\vee}, \omega_{i} \leftrightarrow 2 d h^{i}, \quad \mathfrak{h}^{\vee} \cong \Lambda^{2} \mathfrak{h}^{\vee}, \mathrm{dh}^{i} \leftrightarrow d h^{j k},
$$

where (ijk) is a even permutation of (123). The factor 2 is necessary to identify these as Lie algebras (note that $\Lambda_{+}^{2}$ carries a Lie algebra structure for a Riemannian manifold induced by identification with skew symmetric endomorphisms of TM). We also identify $S^{11}$ in $\Lambda^{2} T^{\vee} X$ and $\mathfrak{h}^{\vee} \otimes T^{\vee} X$ by

$$
\begin{aligned}
& \eta \wedge d x^{5} \leftrightarrow f_{1}=d h^{1} \wedge d x^{6}+d h^{2} \wedge d x^{7}+d h^{3} \wedge d x^{8} . \\
& \eta \wedge d x^{6} \leftrightarrow f_{2}=-d h^{1} \wedge d x^{5}-d h^{2} \wedge d x^{8}+d h^{3} \wedge d x^{7} . \\
& \eta \wedge d x^{7} \leftrightarrow f_{3}=d h^{1} \wedge d x^{8}-d h^{2} \wedge d x^{5}-d h^{3} \wedge d x^{6} . \\
& \eta \wedge d x^{8} \leftrightarrow f_{4}=-d h^{1} \wedge d x^{7}+d h^{2} \wedge d x^{6}-d h^{3} \wedge d x^{5} .
\end{aligned}
$$

We claim, that under these identifications the maps $G\left(\alpha_{i}\right)=\star\left(\alpha_{i} \wedge-\right)$ are given by

\begin{tabular}{ccccc}
\hline & $\mathrm{G}\left(\alpha_{0}\right)$ & $\mathrm{G}\left(\alpha_{1}\right)$ & $\mathrm{G}\left(\alpha_{2}\right)$ & $\mathrm{G}\left(\alpha_{3}\right)$ \\
\hline$\Lambda^{2} \mathfrak{h}^{\vee}$ & $\mathfrak{l}$ & $-\mathfrak{l}$ & \\
$\mathfrak{h}^{\vee} \otimes \mathrm{T}^{\vee} X$ & $\mathrm{pr}_{\mathrm{S}^{20}}$ & $-\mathrm{id}_{\mathrm{S}^{31}}+2 \mathrm{id}_{\mathrm{S}^{11}}$ & $-\mathrm{id}_{\mathrm{S}^{20}}+3 \mathrm{id}_{\mathrm{S}^{11}}$ & \\
$\Lambda^{2} \mathrm{~T}^{\vee} \mathrm{X}$ & & $-2 \mathrm{pr}_{\mathrm{S}^{20}}$ & $-2 \mathrm{id}_{\mathrm{S}^{20}}+\mathrm{id}_{\mathrm{S}^{11}}$ & $\mathrm{id}_{\mathrm{S}^{20}}-\mathrm{id}_{\mathrm{S}^{02}}$ \\
\hline
\end{tabular}

where $\iota$ is the inclusion, pr is the projection onto a factor and $\alpha$ id the action on said space with the given factor $\alpha$.

Let us justify these claims.

Ad $G\left(\alpha_{0}\right):$ First

$$
\left.\mathrm{G}\left(\alpha_{0}\right)\right|_{\Lambda^{2} \mathfrak{h}^{v}}: S^{20} \rightarrow \mathrm{S}^{20} \oplus \mathrm{S}^{31} \oplus \mathrm{S}^{11}
$$

By Schur's Lemma $G\left(\alpha_{0}\right)$ can only map into the $S^{20}$ components. We calculate explicitly, that $G\left(\alpha_{1}\right)$ is the isomorphism of the $S^{20}$ components given by the identification above. Indeed,

$$
\mathrm{G}\left(\alpha_{0}\right)\left(\star_{3} d h^{i}\right)=\eta \wedge d h^{i}
$$

Similarly,

$$
\left.\mathrm{G}\left(\alpha_{0}\right)\right|_{\mathfrak{h}^{\vee} \otimes \mathrm{T}^{\vee} \mathrm{X}}: S^{20} \oplus \mathrm{S}^{31} \oplus \mathrm{S}^{11} \rightarrow \mathrm{S}^{20},
$$

we conclude that the kernel of $G\left(\alpha_{0}\right)$ includes $S^{31}$ and $S^{11}$ components. Again a direct computation shows that $\eta \wedge d h^{i}$ gets mapped to $\star_{3} d h^{i}$. 
Ad $G\left(\alpha_{1}\right)$ : Let us now look at $G\left(\alpha_{1}\right)$.

$$
\left.\mathrm{G}\left(\alpha_{1}\right)\right|_{\Lambda^{2} \mathfrak{h}^{\vee}}: \mathrm{S}^{20} \rightarrow \mathrm{S}^{20} \oplus \mathrm{S}^{02} \oplus \mathrm{S}^{11},
$$

has its image in $S^{20}$. We calculate that

$$
\left.\mathrm{G}\left(\alpha_{1}\right)\right|_{\Lambda^{2} \mathfrak{h} \vee}\left(\star_{3} d h^{i}\right)=-\omega_{i},
$$

which shows that $G\left(\alpha_{1}\right)$ is just twice the negative of the identification of the two $S^{20}$ components.

$$
\left.\mathrm{G}\left(\alpha_{1}\right)\right|_{\Lambda^{2} \mathrm{~T} \vee}\left(\omega_{i}\right)=-\star_{3} d h^{i},
$$

so is the negative of the projection up to the identification.

Finally

$$
\left.\mathrm{G}\left(\alpha_{1}\right)\right|_{\mathfrak{h}^{\vee} \otimes \mathrm{T}^{\vee} \mathrm{X}}
$$

if follows immediately that $S^{20}$ gets mapped to 0 , because of the $\eta$ factor. For $S^{31} \oplus S^{11}$, it is fastest to realize that $F \alpha_{1}$ preserves the components of $f_{i}$. So the map decomposes into three 3-dimensional maps which can be easily diagonalized. Each one of the three has two eigenvalues given by $2,-1$ where -1 comes with multiplicity 2 . For dimensional reasons $G\left(\alpha_{1}\right)$ then has to act as described in the table.

Ad $G\left(\alpha_{2}\right)$ :

$$
\left.\mathrm{G}\left(\alpha_{2}\right)\right|_{\wedge^{2} \mathrm{~T}^{\vee} \mathrm{X}}\left(\omega_{i}\right)=-\eta \wedge \star_{3} \mathrm{dh}^{i} .
$$

The basis of $S^{11}$ has been chosen so that $\mathrm{G}\left(\alpha_{2}\right)$ is the identity. Furthermore we calculate

$$
\left.\mathrm{G}\left(\alpha_{2}\right)\right|_{\mathfrak{h} \vee \otimes T^{\vee} X}\left(\eta \wedge \star_{3} \mathrm{dh}^{i}\right)=-\omega_{\mathfrak{i}}
$$

and

$$
\left.\mathrm{G}\left(\alpha_{2}\right)\right|_{\mathfrak{h}^{\vee} \otimes \mathrm{T}^{\vee} \mathrm{X}}\left(f_{\mathfrak{i}}\right)=3 \eta \wedge d x^{i},
$$

verifying the claim.

$\operatorname{Ad} G\left(\alpha_{3}\right)$ :

$$
\mathrm{G}\left(\alpha_{3}\right): \mathrm{S}^{20} \oplus \mathrm{S}^{02} \oplus \mathrm{S}^{11} \rightarrow \mathrm{S}^{20} \oplus \mathrm{S}^{02} \oplus \mathrm{S}^{11},
$$

and these are the eigenspaces of the map to the eigenvalues $1,-1,0$. Indeed, if $\omega \in S^{11}$, then $G\left(\alpha_{3}\right)(\omega)=0$, since $\alpha_{3}$ has the factor $\eta$. If $\omega \in S^{20}$ or $S^{02}$, then by Lemma 2.9

$$
\mathrm{G}\left(\alpha_{3}\right)(\omega)=\star\left(\alpha_{3} \wedge \omega\right)=\star_{4} \alpha \wedge \star_{4} \omega=\star_{4} \omega,
$$

and $S^{20}$ and $S^{02}$ are the eigenspaces to the eigenvalues 1 and -1 of $\star_{4}$ respectively. 
The Equations in $\Lambda^{2} \mathfrak{h}^{\vee}$ The equations in $\Lambda^{2} \mathfrak{h}^{\vee}$ is given by

$$
-\frac{1}{2} \varphi \circ[\tilde{\mathrm{B}} \wedge \tilde{\mathrm{B}}]-\frac{1}{2}[\varphi \circ \tilde{\mathrm{B}} \wedge \varphi \circ \tilde{\mathrm{B}}]=\star_{8}\left(\alpha_{0} \wedge\left(\mathrm{d}_{\mathrm{a}} \varphi \stackrel{\circ}{\wedge} \tilde{\mathrm{B}}\right)\right)+\star_{8}\left(\alpha_{1} \wedge \mathrm{F}^{\mathrm{a}}\right),
$$

where the first term comes from the Maurer-Cartan equations for $\varphi \circ \mathrm{d} \tilde{\mathrm{B}}$.

We know that $G\left(\alpha_{1}\right)\left(F^{a}\right)=-F_{+}^{a}$ up to the identification, i.e.

$$
\star_{8}\left(\alpha_{1} \wedge F^{a}\right)=-F_{+, 1}^{a} d^{23}-F_{+, 2}^{a} d h^{31}-F_{+, 3}^{a} d^{12} .
$$

From the table $G\left(\alpha_{0}\right)$ is the projection onto the $S^{20}$ component of $d_{a} \varphi \stackrel{\circ}{\wedge} \tilde{B}$, which is given by

$$
\operatorname{pr}_{\mathrm{S}^{20}}\left(\mathrm{~d}_{\mathrm{a}} \varphi \stackrel{\circ}{\wedge} \tilde{\mathrm{B}}\right)=\sum_{i=1}^{2} \nabla_{4} \varphi_{\mathrm{i}} \eta \wedge \mathrm{dh}^{\mathrm{i}},
$$

so that

$$
\star_{8}\left(\alpha_{0} \wedge\left(\mathrm{d}_{\mathrm{a}} \varphi \stackrel{\circ}{\wedge} \tilde{\mathrm{B}}\right)\right)=\nabla_{4}^{\mathrm{a}} \varphi_{1} \mathrm{dh}^{23}+\nabla_{4}^{\mathrm{a}} \varphi_{2} \mathrm{dh}^{31}+\nabla_{4}^{\mathrm{a}} \varphi_{3} \mathrm{dh}^{12} .
$$

As before, the Maurer-Cartan equations yield a 2B and finally

$$
-\frac{1}{2}[\varphi \circ \tilde{B} \wedge \varphi \circ \tilde{B}]=-\sum_{1 \leqslant i<j \leqslant 3}\left[\varphi_{i}, \varphi_{j}\right] d h^{i j}
$$

This gives

$$
\begin{aligned}
0 & =\left[\varphi_{1}, \varphi_{2}\right]-F_{+, 3}^{\mathrm{a}}+\nabla_{4}^{\mathrm{a}} \varphi_{3} \\
0 & =\left[\varphi_{2}, \varphi_{3}\right]-F_{+, 1}^{\mathrm{a}}+\nabla_{4}^{\mathrm{a}} \varphi_{1} \\
0 & =\left[\varphi_{3}, \varphi_{1}\right]-F_{+, 2}^{\mathrm{a}}+\nabla_{4}^{\mathrm{a}} \varphi_{2}
\end{aligned}
$$

which is equivalent to

$$
\mathrm{F}_{+}^{\mathrm{a}}-\nabla_{\mathrm{Y}}^{\mathrm{a}} \mathrm{B}-2 \mathrm{~B}-\sigma(\mathrm{B}, \mathrm{B})=0
$$

The Equations in $\mathfrak{h}^{\vee} \otimes T^{\vee} X:$

$$
-\mathrm{d}_{\mathrm{a}} \varphi \stackrel{\circ}{\wedge} \tilde{\mathrm{B}}=\star_{8}\left(\alpha_{0} \wedge \frac{1}{2}(\varphi \circ[\tilde{\mathrm{B}} \wedge \tilde{\mathrm{B}}]+[\varphi \circ \tilde{\mathrm{B}} \wedge \varphi \circ \tilde{\mathrm{B}}])+\alpha_{1} \wedge\left(\mathrm{d}_{\mathrm{a}} \varphi \stackrel{\circ}{\wedge} \tilde{\mathrm{B}}\right)+\alpha_{2} \wedge \mathrm{F}^{\mathrm{a}}\right)
$$

Here we have three equations, in $S^{20}, S^{31}$ and $S^{11}$ of dimension 3,8 and 4 . Let us first focus on the equation in $S^{31}$. Looking at the table we see that only $G\left(\alpha_{1}\right)$ has values in $S^{31}$, so the equation becomes

$$
-\operatorname{pr}_{S^{31}}\left(d_{a} \varphi \stackrel{\circ}{\wedge} \tilde{B}\right)=G\left(\alpha_{1}\right) \circ \operatorname{pr}_{S^{31}}\left(d_{a} \varphi \stackrel{\circ}{\wedge} \tilde{B}\right),
$$


which is trivially satisfied as $G\left(\alpha_{1}\right)$ acts with eigenvalue -1 on $S^{31}$. Now let us turn to the equation in $S^{20} . G\left(\alpha_{1}\right)$ contributes nothing to this equation, $G\left(\alpha_{0}\right)$ is simply the inclusion and $G\left(\alpha_{2}\right)$ acts by multiplication of -1 . Hence the equation becomes

$$
\operatorname{pr}_{\mathrm{S}^{20}}\left(\mathrm{~d}_{\mathrm{a}} \varphi \stackrel{\circ}{\wedge} \tilde{\mathrm{B}}++\frac{1}{2} \varphi \circ[\tilde{\mathrm{B}} \wedge \tilde{\mathrm{B}}]+\frac{1}{2}[\varphi \circ \tilde{\mathrm{B}} \wedge \varphi \circ \tilde{\mathrm{B}}]-\mathrm{F}^{\mathrm{a}}\right)=0
$$

Now we already now that $\mathrm{pr}_{\mathrm{S}^{20}} \mathrm{~F}^{\mathrm{a}}=\mathrm{F}_{+}^{\mathrm{a}}$,

$$
\operatorname{pr}_{\mathrm{S}^{20}}\left(\mathrm{~d}_{\mathrm{a}} \varphi \stackrel{\circ}{\wedge} \tilde{\mathrm{B}}\right)=\nabla_{\mathrm{Y}}^{\mathrm{a}} \mathrm{B},
$$

and

$$
\frac{1}{2} \varphi \circ[\tilde{\mathrm{B}} \wedge \tilde{\mathrm{B}}]+\frac{1}{2}[\varphi \circ \tilde{\mathrm{B}} \wedge \varphi \circ \tilde{\mathrm{B}}]=2 \mathrm{~B}+\sigma(\mathrm{B}, \mathrm{B}),
$$

which shows that the equation is equivalent to (5.114).

Finally let us look at the equation in $S^{11}$. The equation is given by

$$
0=\operatorname{pr}_{\mathrm{S}^{11}}\left(\mathrm{~d}_{\mathrm{a}} \varphi \stackrel{\circ}{\wedge} \tilde{\mathrm{B}}+2 \mathrm{~d}_{\mathrm{a}} \varphi \stackrel{\circ}{\wedge} \tilde{\mathrm{B}}+\mathrm{F}^{\mathrm{a}}\right)=\operatorname{pr}_{\mathrm{S}^{11}}\left(3 \mathrm{~d}_{\mathrm{a}} \varphi \stackrel{\circ}{\wedge} \tilde{\mathrm{B}}+\mathrm{F}^{\mathrm{a}}\right) .
$$

This gives the same equation as 5.136 .

The Equations in $\wedge^{2} T^{\vee} X$ :

$$
-\mathrm{F}^{\mathrm{a}}=\star_{8}\left(\alpha_{1} \wedge\left(\frac{1}{2} \varphi \circ[\tilde{\mathrm{B}}, \tilde{\mathrm{B}}]+\frac{1}{2}[\varphi \circ \tilde{\mathrm{B}} \wedge \varphi \circ \tilde{\mathrm{B}}]\right)+\alpha_{2} \wedge \mathrm{d}_{\mathrm{a}} \varphi \stackrel{\circ}{\wedge} \tilde{\mathrm{B}}+\alpha_{3} \wedge \mathrm{F}^{\mathrm{a}}\right)
$$

Again there are three components, $S^{20}, S^{02}$ and $S^{11}$ of dimension 3,3 and 4 . The $S^{02}$ component is given by

$$
-\mathrm{F}_{-}^{\mathrm{a}}=\mathrm{G}\left(\alpha_{3}\right)\left(\mathrm{F}_{-}^{\mathrm{a}}\right)=-\mathrm{F}_{-}^{\mathrm{a}},
$$

again a tautology. The $S^{20}$ component is

$$
-\mathrm{F}_{+}^{\mathrm{a}}=-\varphi[\tilde{\mathrm{B}} \wedge \tilde{\mathrm{B}}]-[\varphi \circ \tilde{\mathrm{B}} \wedge \varphi \circ \tilde{\mathrm{B}}]-2 \operatorname{pr}_{\mathrm{S}^{20}}\left(\mathrm{~d}_{\mathrm{a}} \varphi \stackrel{\circ}{\wedge} \tilde{\mathrm{B}}\right)+\mathrm{F}_{+}^{\mathrm{a}}
$$

or

$$
0=-2 B-2 \sigma(B, B)-2 \nabla_{Y}^{a} B+2 F_{+}^{a} .
$$

Finally the $S^{11}$ component is given by

$$
-\mathrm{F}_{4}^{\mathrm{a}}=3 \operatorname{pr}_{\mathrm{S}^{11}}\left(\mathrm{~d}_{\mathrm{a}} \varphi \stackrel{\circ}{\wedge} \tilde{\mathrm{B}}\right) .
$$

Now it is easy to see that

$$
F_{4}^{a}=\sum_{i=5}^{8} F_{1 i}^{a} \eta \wedge d x^{i}
$$


Identifying $S^{11}$ with $S^{11} \subset T^{\vee} X$ via $\iota_{Y}$, this gives $\iota_{Y} F^{a}$.

Now using the basis $f_{1}, \ldots f_{4}$, we see that

$$
\begin{aligned}
\operatorname{pr}_{\mathrm{S}^{11}}\left(\mathrm{~d}_{\mathrm{a}} \varphi \stackrel{\circ}{\wedge} \tilde{\mathrm{B}}\right)= & \frac{1}{3} \sum_{i=1}^{4} \mathrm{f}_{\mathrm{i}} \mathrm{f}_{\mathrm{i}}^{\vee}\left(\mathrm{d}_{\mathrm{a}} \varphi \stackrel{\circ}{\wedge} \tilde{\mathrm{B}}\right) \\
= & \frac{\mathrm{f}_{1}}{3}\left(\nabla_{6}^{\mathrm{a}} \varphi_{1}+\nabla_{7}^{\mathrm{a}} \varphi_{2}+\nabla_{8}^{\mathrm{a}} \varphi_{3}\right) \\
& +\frac{f_{2}}{3}\left(-\nabla_{5}^{\mathrm{a}} \varphi_{1}-\nabla_{8}^{\mathrm{a}} \varphi_{2}+\nabla_{7}^{\mathrm{a}} \varphi_{3}\right) \\
& +\frac{\mathrm{f}_{3}}{3}\left(\nabla_{8}^{\mathrm{a}} \varphi_{1}-\nabla_{5}^{\mathrm{a}} \varphi_{2}-\nabla_{6}^{\mathrm{a}} \varphi_{3}\right) \\
& +\frac{\mathrm{f}_{4}}{3}\left(-\nabla_{7}^{\mathrm{a}} \varphi_{1}+\nabla_{6}^{\mathrm{a}} \varphi_{2}-\nabla_{5}^{\mathrm{a}} \varphi_{3}\right) .
\end{aligned}
$$

Mapping this over yields

$$
\begin{aligned}
\mathrm{G}\left(\alpha_{2}\right) \circ \operatorname{pr}_{\mathrm{S} 11}\left(\mathrm{~d}_{\mathrm{a}} \varphi \stackrel{\circ}{\wedge}\right)= & \eta \wedge \mathrm{d} x^{5}\left(\nabla_{6}^{\mathrm{a}} \varphi_{1}+\nabla_{7}^{\mathrm{a}} \varphi_{2}+\nabla_{8}^{\mathrm{a}} \varphi_{3}\right) \\
& +\eta \wedge \mathrm{d} x^{6}\left(-\nabla_{5}^{\mathrm{a}} \varphi_{1}-\nabla_{8}^{\mathrm{a}} \varphi_{2}+\nabla_{7}^{\mathrm{a}} \varphi_{3}\right) \\
& +\eta \wedge \mathrm{d} x^{7}\left(\nabla_{8}^{\mathrm{a}} \varphi_{1}-\nabla_{5}^{\mathrm{a}} \varphi_{2}-\nabla_{6}^{\mathrm{a}} \varphi_{3}\right) \\
& +\eta \wedge \mathrm{d} x^{8}\left(-\nabla_{7}^{\mathrm{a}} \varphi_{1}+\nabla_{6}^{\mathrm{a}} \varphi_{2}-\nabla_{5}^{\mathrm{a}} \varphi_{3}\right) .
\end{aligned}
$$

We will denote this with $-\delta_{+}^{a} B$ (the notation will be explained in section 6.3). Then the equation is

$$
\iota_{Y} \mathrm{~F}^{\mathrm{a}}-\delta_{+}^{\mathrm{a}} \mathrm{B}=0 .
$$

Putting this together shows that we have the perturbed Haydys-Witten equations,

$$
\begin{array}{r}
\iota_{Y} F^{a}-\delta_{+}^{a} B=0 \\
F_{+}^{a}-\nabla_{Y}^{a} B-2 B-\sigma(B, B)=0 .
\end{array}
$$

If we simplify this to an action by $\mathbb{R}^{3}$ so that $d \tilde{B}=0$, then the resulting equations are given by the Haydys-Witten equations

$$
\begin{array}{r}
\mathfrak{l}_{Y} F^{a}-\delta_{+}^{a} B=0 \\
F_{+}^{a}-\nabla_{Y}^{a} B-\sigma(B, B)=0 .
\end{array}
$$

Remark 5.20. If we simplify the action to be $\mathbb{R}^{3}$, then by Corollary 2.64 the underlying manifold has a hypo structure. In this case, the $\Lambda_{+}^{2}$ representation decomposes into three trivial representations (compare to Proposition 2.44) and the second equation decouples into the three Nahm equations with the additional term $F_{+}^{a}$. 


\section{Chapter 6}

\section{Dimensionally Reduced Gauge Theories}

In this chapter we will collect known facts about the dimensionally reduced equations that we need later on. Most of this chapter can be found scattered through the literature. We will introduce a common notation and make consistent choices where they are needed to formulate the results in Chapter 8

\subsection{Nahm Equations}

Throughout this section, fix a Lie Group G with an Ad-invariant scalar product $\langle-,-\rangle$ on the Lie Algebra $\mathfrak{g}$.

\subsubsection{The Equations}

Definition 6.1. A Nahm datum is a quadruple of maps

$$
\mathrm{T}_{i}: \mathrm{I}=(-1,1) \rightarrow \mathfrak{g}, \quad i \in\{0,1,2,3\} .
$$

Remark 6.2. Sometimes it will be convenient to combine these to

$$
\mathrm{T}: \mathrm{I} \rightarrow \mathbb{H} \otimes \mathfrak{g},
$$

where $\operatorname{Re}(\mathbf{T})=\mathrm{T}_{0}$.

Notation 6.3. We introduce the following notation,

$$
W_{N}=\mathfrak{C}^{\infty}(\mathrm{I}, \mathbb{H} \otimes \mathfrak{g}) \quad \text { and } \quad \mathrm{U}_{\mathrm{N}}=\mathfrak{C}^{\infty}\left(\mathrm{I}, \operatorname{Im}(\mathbb{H}) \otimes \mathfrak{g}^{\vee}\right) .
$$

Definition 6.4. A Nahm datum satisfies the Nahm Equations if

$$
\dot{\mathrm{T}}_{i}+\left[\mathrm{T}_{0}, \mathrm{~T}_{i}\right]+\frac{1}{2} \sum_{j, k=1}^{3} \varepsilon_{i j k}\left[\mathrm{~T}_{j}, \mathrm{~T}_{k}\right]=0 \quad i \in\{1,2,3\} .
$$


Define the map

$$
\mu_{N}: W_{N} \rightarrow U_{N}, \quad\left(T_{0}, T_{1}, T_{2}, T_{3}\right) \mapsto\left(N_{1}, N_{2}, N_{3}\right),
$$

where $N_{i}=N_{i}\left(T_{0}, T_{1}, T_{2}, T_{3}\right)$ are the three Nahm equations.

Remark 6.5. From the reduction theory, we know that $T_{0}$ is a connection and $T_{1}, T_{2}, T_{3}$ are sections of the adjoint bundle. Using this we can write the equations as

$$
\mathrm{d}_{\mathrm{T}_{0}} \mathrm{~T}_{i}+\frac{1}{2} \sum_{j, k=1}^{3} \varepsilon_{i j k}\left[\mathrm{~T}_{j}, \mathrm{~T}_{k}\right]=0 \quad i \in\{1,2,3\} .
$$

Remark 6.6. The gauge group of the bundle $\mathcal{G}_{N}=\mathfrak{C}^{\infty}(\mathrm{I}, \mathrm{G})$ (from now on just $\mathcal{G}$ in this section) acts on both sections and connections in a natural way given by

$$
g \cdot T_{0}=\operatorname{Ad}_{g} T_{0}+\left(g^{-1}\right)^{\star} \omega_{G}, \quad T_{i} \cdot g=A d_{g^{-1}} T_{i},
$$

where $\omega_{G}$ is the Maurer Cartan form of G. For a matrix group the action is given by g. $\mathrm{T}_{0}=g \mathrm{~T}_{0} \mathrm{~g}^{-1}-\dot{\mathrm{g}} \mathrm{g}^{-1}$.

Since $[-,-]$ is Ad-equivariant and $d_{g \cdot T_{0}} g \cdot T_{i}=\operatorname{Ad}_{g} d_{T_{0}} T_{i}$ by the standard formulas,

$$
\mu_{\mathrm{N}}(\mathrm{g} \cdot \mathbf{T})=\operatorname{Ad}_{\mathrm{g}} \circ \mu_{\mathrm{N}}(\mathbf{T}) .
$$

The equivariance of $\mu_{N}$ shows that the kernel of $\mu_{N}$ is invariant; so the gauge group acts on solutions of the Nahm Equations.

Remark 6.7. The gauge group can be used to eliminate the connection completely from the problem. The trick is to choose a special gauge which is called temporal gauge (sometimes Weyl gauge; technically we are looking at a slice of the gauge action). In the case of the matrix group this can be easily seen by requiring $g . T_{0}=0$ and solving the linear ordinary differential equation

$$
\dot{g}=g_{0}, \quad g(0)=g_{0} \in \mathfrak{g},
$$

on I. For general G, one can use parallel translation to achieve the same. Then the Nahm equations become

$$
\left\{\begin{array}{l}
\dot{\mathrm{T}}_{1}+\left[\mathrm{T}_{2}, \mathrm{~T}_{3}\right]=0 \\
\dot{\mathrm{T}}_{2}+\left[\mathrm{T}_{3}, \mathrm{~T}_{1}\right]=0 \\
\dot{\mathrm{T}}_{3}+\left[\mathrm{T}_{1}, \mathrm{~T}_{2}\right]=0
\end{array}\right.
$$

and the only remaining action of the gauge group is via constant gauges (i.e. by choosing a different initial condition go). 


\subsubsection{Boundary Conditions}

From now on fix $G=\mathbf{U}(k)$, for $k \in \mathbb{N}$.

Definition 6.8. Fix $k \in \mathbb{N}$. Let $\rho_{1}, \rho_{2}, \rho_{3} \in \mathfrak{g}$ determine an irreducible representation of $\mathfrak{s u}(2)$. We say that a solution to the Nahm Equations satisfies the boundary conditions $\mathrm{NC}_{\mathrm{k}}$ if,

- $\mathrm{T}_{0}$ is smooth on $\overline{\mathrm{I}}=[-1,1]$,

- for $i \neq 0$ and $t \rightarrow 1,\left\|(t-1) T_{i}-\rho_{i}\right\| \in \mathcal{O}(t-1)$, and

- for $i \neq 0$ and $t \rightarrow-1,\left\|(t+1) T_{i}-\rho_{i}\right\| \in \mathcal{O}(t+1)$.

It may seem strange that the choice of $\rho_{i}$ is not denoted for the boundary conditions $\mathrm{NC}_{\mathrm{k}}\left(\right.$ e.g. $\left.\mathrm{NC}_{\mathrm{k}}^{\rho}\right)$. It is however true, that all irreducible representations of $\mathfrak{s u}(2)$ in $\mathfrak{u}(\mathrm{k})$ are conjugated. Hence given two different irreducible representations $\rho, \rho^{\prime}$, we can pick an element $g \in \mathcal{G}$ from $g(-1)=1$ to $g(1)=u$, where $u \in \mathbf{U}(k)$ conjugates $\operatorname{Ad}_{\mathfrak{u}} \rho=\rho^{\prime}$. Then the adjoint action $\mathrm{Ad}_{\mathrm{g}}$ maps solutions with $\rho$ at the right boundary to solutions with $\rho^{\prime}$ at the right boundary. This shows that the actual choice of representation is not important.

This observation also shows that we have to restrict to a smaller gauge group if we want the boundary conditions to be invariant under the gauge action.

Definition 6.9. Define the space of framed gauge transformations as the space

$$
\mathcal{G}_{0}=\{g \in \mathcal{G} \mid\|g-1\| \in \mathcal{O}(t-1),\|g-1\| \in \mathcal{O}(t+1)\},
$$

where the first requirement is for $t \rightarrow 1$ and the second one for $t \rightarrow-1$.

Proposition 6.10. The space $\mathcal{G}_{0}$ acts on solutions of the Nahm equations satisfying the boundary conditions $N C_{k}$ for $k \in \mathbb{N}$.

Proof. We will only show the claim for $t=1$ and a fixed $i \in\{1,2,3\}$, the other cases are similar. The boundary condition $\mathrm{NC}_{\mathrm{k}}$ is equivalent to

$$
\mathrm{T}_{i}(\mathrm{t})=\frac{\rho_{i}}{\mathrm{t}-1}+\mathrm{b}_{\mathfrak{i}}(\mathrm{t}), \quad\left\|\mathrm{b}_{\mathfrak{i}}\right\| \in \mathcal{O}(1) .
$$

The condition on $g \in \mathcal{G}_{0}$ is that $g(t)=1+c(t)$, where $\|c(t)\| \in \mathcal{O}(t-1)$. Without loss of generality we may assume $\|c(t)\|<1$, so that

$$
g^{-1}(t)=\sum_{k \in \mathbb{N}_{0}}(-c(t))^{k} .
$$

Therefore the action of $g$ on $T_{i}$ is given by

$$
\operatorname{Ad}_{g} T_{i}(t)=\frac{\rho_{i}}{t-1}+\underbrace{\frac{\left[c(t), \rho_{i}\right]}{t-1}}_{\|-\|=\mathcal{O}(1)}+\underbrace{\frac{(1+c(t)) \rho_{i} \sum_{k \geqslant 2}(-c(t))^{k}}{t-1}}_{\|-\|=\mathcal{O}(t-1)}+\underbrace{g b_{i}(t) g^{-1}}_{\|-\|=\mathcal{O}(1)}
$$




\subsubsection{The Moduli Space}

Notation 6.11. Denote by $F_{N}$ the subspace of $W_{N}$ that satisfies the boundary conditions $N_{k}$ for some $k \in \mathbb{N}$ ( $F_{N}^{k}$ if we want to fix the $k \in \mathbb{N}$ ). Further fix the notation

$$
\mathrm{V}_{\mathrm{N}}=\mathfrak{C}^{\infty}(\overline{\mathrm{I}}, \mathfrak{u}(\mathrm{k}))^{4}
$$

Definition 6.12. Fix $k \in \mathbb{N}$. With $N_{k}$ we denote the intersection of $\mu_{N}^{-1}(0)$ with $F_{N}$, i.e. solutions of the Nahm equations for $G=\mathbf{U}(k)$, subject to the boundary condition $\mathrm{NC}_{\mathrm{k}}$. The framed gauge group $\mathcal{G}_{0}$ acts freely on this space, and the quotient is a $4 \mathrm{k}$ dimensional hyperkähler manifold [AH88] denoted by

$$
\mathcal{N}_{k}=N_{k} / \mathcal{G}_{0}
$$

Remark 6.13. The Nahm equations have a close relative, the self-dual Nahm equations. In the literature, these are often also simply called Nahm equations. The modification is changing the orientation of $\mathbb{R}^{4}$ i.e. swapping anti self-dual instantons with anti-self dual instantons. Section 5.2.3 shows that the corresponding Nahm equations are given by

$$
\nabla_{\mathrm{t}} \mathrm{T}_{\mathrm{i}}-\frac{1}{2} \sum_{j, k=1}^{3} \varepsilon_{i j k}\left[\mathrm{~T}_{j}, \mathrm{~T}_{\mathrm{k}}\right]=0, \quad i \in\{1,2,3\} .
$$

We define everything else similarly to the regular Nahm equations and denote the space by $\mathcal{N}_{k}^{(s)}=N_{k}^{(s)} / \mathcal{G}_{0}$. Note that $N_{k}^{(s)} \cong N_{k}$ by mapping $\left(T_{0}, T_{1}, T_{2}, T_{3}\right) \rightarrow\left(T_{0}, T_{1},-T_{2}, T_{3}\right)$.

Lemma 6.14. $\mathrm{F}_{\mathrm{N}}$ is an affine space over $\mathrm{V}_{\mathrm{N}}$.

Proof. The boundary condition ensures that the difference of two solutions $T_{i}-\tilde{T}_{i}$ is bounded for $i \in\{1,2,3\}$, hence extensible to the closed interval.

Proposition 6.15. Let $\mathrm{c}=\left(\mathrm{T}_{0}, \mathrm{~T}_{1}, \mathrm{~T}_{2}, \mathrm{~T}_{3}\right) \in \mathrm{N}_{\mathrm{k}}^{(\mathrm{s})}$. Then the space $\mathrm{T}_{\mathrm{c}} \mathrm{N}_{\mathrm{k}}^{(\mathrm{s})}$ consists of quadruples $\left(t_{0}, t_{1}, t_{2}, t_{3}\right)$ where

$$
t_{i}: \bar{I} \rightarrow \mathfrak{u}(k), \quad i \in\{0,1,2,3\},
$$

that satisfy the linearized (self dual) Nahm equations

$$
\left\{\begin{array}{l}
0=\dot{t}_{1}+\left[t_{0}, T_{1}\right]+\left[T_{0}, t_{1}\right] \pm\left[t_{2}, T_{3}\right] \pm\left[T_{2}, t_{3}\right] \\
0=\dot{t}_{2}+\left[t_{0}, T_{2}\right]+\left[T_{0}, t_{2}\right] \pm\left[t_{3}, T_{1}\right] \pm\left[T_{3}, t_{1}\right] \\
0=\dot{t}_{3}+\left[t_{0}, T_{3}\right]+\left[T_{0}, t_{3}\right] \pm\left[t_{1}, T_{2}\right] \pm\left[T_{1}, t_{2}\right]
\end{array}\right.
$$

where the upper sign is for the case of $\mathrm{N}_{\mathrm{k}}$, the lower sign for the case of $\mathrm{N}_{\mathrm{k}}^{(\mathrm{s})}$.

Proof. The condition of $\mathrm{N}_{\mathrm{k}}^{(s)}$ is that the Nahm equations are satisfied. This is described via the linearized Nahm equations 6.19. 
Remark 6.16. To pass form $\mathrm{N}_{\mathrm{k}}^{(s)}$ to $\mathcal{N}_{\mathrm{k}}^{(s)}$, we quotient out the gauge group $\mathcal{G}_{0}$. In order to describe the space $T_{[c]} \mathcal{N}_{k}^{(s)}$ for $c \in N_{k}^{(s)}$, we need to pick an orthogonal complement in $T_{c} N_{k}^{(s)}$ to the infinitesimal generators of $\mathcal{G}_{0} \triangleleft \mathrm{N}_{k}^{(s)}$.

Definition 6.17. Define a metric on $\mathrm{N}_{\mathrm{k}}^{(s)}$ via

$$
\left\langle\left(t_{0}, t_{1}, t_{2}, t_{3}\right),\left(s_{0}, s_{1}, s_{2}, s_{3}\right)\right\rangle=\int_{I} \sum_{i=0}^{3}\left\langle t_{i}, s_{i}\right\rangle d t,
$$

where $\left(t_{i}\right)$ and $\left(s_{i}\right)$ are tangent vectors as in proposition 6.15 and $\langle t, s\rangle$ on $\mathfrak{u}(k)$ is given by $-\frac{1}{2} \operatorname{tr}(\mathrm{ts})$.

Remark 6.18. Note that $\langle\mathrm{t}, \mathrm{s}\rangle=-\frac{1}{2} \operatorname{tr}(\mathrm{ts})$ is almost the negative of the Killing form, the difference being the normalization and the fact that it is not zero on $i \mathbb{l} \in \mathfrak{u}(k)$. Notice that this implies that $\mathrm{ad}_{\mathrm{g}}$ is skew-symmetric with respect to $\langle-,-\rangle$ for all $g \in \mathfrak{u}(\mathrm{k})$.

Lemma 6.19. The induced action of the gauge group $\mathcal{G}$ on the tangent space $\mathrm{TN}_{\mathrm{k}}^{(\mathrm{s})}$ is given by

$$
g .\left(t_{0}, t_{1}, t_{2}, t_{3}\right)=\left(\operatorname{Ad}_{g} t_{0}, \operatorname{Ad}_{g} t_{1}, \operatorname{Ad}_{g} t_{2}, \operatorname{Ad}_{g} t_{3}\right) .
$$

Proof. We need to simply differentiate the map $g: N_{k} \rightarrow N_{k}$ for a fixed $g \in \mathcal{G}$. Since $g$ acts linear on $T_{1}, T_{2}$ and $T_{3}$ and affine linear on $T_{0}$, these formulas follow immediately.

Proposition 6.20. The metric is invariant under the gauge action $\mathcal{G}_{0} \frown \mathrm{N}_{\mathrm{k}}^{(\mathrm{s})}$.

Proof. This follows immediately since the Killing form is Ad-invariant.

Lemma 6.21. The infinitesimal action of the Lie Algebra of $\mathcal{G}_{0}$,

$$
\operatorname{Lie}\left(\mathcal{G}_{0}\right)=\{\xi \in \Gamma(\bar{I}, \operatorname{Ad}(\mathrm{P})) \mid \xi(-1)=0=\xi(1)\}
$$

on $\mathrm{N}_{\mathrm{k}}^{(\mathrm{s})}$ is given by

$$
\mathrm{K}^{\xi}=\left(-\mathrm{d}_{\mathrm{T}_{0}} \xi,\left[\xi, \mathrm{T}_{1}\right],\left[\xi, \mathrm{T}_{2}\right],\left[\xi, \mathrm{T}_{3}\right]\right) .
$$

Proof. Note that the exponential map $\operatorname{Lie}\left(\mathcal{G}_{0}\right) \rightarrow \mathcal{G}_{0}$ is given by

$$
\xi: \overline{\mathrm{I}} \rightarrow \mathfrak{u}(\mathrm{k}) \quad \mapsto \quad \exp (\xi): \overline{\mathrm{I}} \rightarrow \mathbf{U}(\mathrm{k}), \quad \exp (\xi)=\exp _{\mathbf{U}(\mathrm{k})} \circ \xi .
$$

Then acting by $g_{s}=\exp (s \xi)$ on $T_{0}$ and differentiating yields

$$
g_{s} \cdot T_{0}=g_{s} T_{0} g_{s}^{-1}-\left.\frac{d g_{s}}{d t} g_{s}^{-1} \Rightarrow \frac{d\left(g_{s} \cdot T_{0}\right)}{d s}\right|_{s=0}=\xi T_{0}-T_{0} \xi-\frac{d \xi}{d t} .
$$

a similar, but simpler, calculation gives

$$
\frac{d\left(g_{s} \cdot T_{i}\right)}{d t}=\left[\xi, T_{i}\right], \quad i \in\{1,2,3\}
$$


Proposition 6.22. A tangent vector $\left(\mathrm{t}_{0}, \mathrm{t}_{1}, \mathrm{t}_{2}, \mathrm{t}_{3}\right)$ to $\mathrm{c}=\left(\mathrm{T}_{0}, \mathrm{~T}_{1}, \mathrm{~T}_{2}, \mathrm{~T}_{3}\right) \in \mathrm{N}_{\mathrm{k}}^{(\mathrm{s})}$ is orthogonal to the infinitesimal generators of $\mathcal{G}_{0} \frown \mathrm{N}_{\mathrm{k}}^{(\mathrm{s})}$ with respect to the metric 6.20 if and only if

$$
0=\dot{t}_{0}+\sum_{i=0}^{3}\left[T_{i}, t_{i}\right] .
$$

Proof. By the last Lemma, the orthogonality is equivalent to

$$
0=-\int_{I}\left\langle t_{0}, d \xi\right\rangle d t+\int_{I} \sum_{i=0}^{3}\left\langle\left[\xi, T_{i}\right], t_{i}\right\rangle d t
$$

Using the fact that $\xi$ vanishes at the boundary, we can use partial integration to show that

$$
\int_{I}\left\langle t_{0}, d \xi\right\rangle d t=-\int_{I}\left\langle\dot{t}_{0}, \xi\right\rangle d t
$$

Recalling that the Killing form (and also multiples of it) are ad-skew-symmetric, $\left\langle\operatorname{ad}_{x} y, z\right\rangle=-\left\langle y, \operatorname{ad}_{x} z\right\rangle$, we see that the orthogonality is equivalent to

$$
0=\int_{I}\left\langle\dot{t}_{0}, \xi\right\rangle d t+\int_{I} \sum_{i=0}^{3}\left\langle\left[T_{i}, t_{i}\right], \xi\right\rangle d t
$$

which is satisfied for all $\xi$ if and only if

$$
0=\dot{t}_{0}+\sum_{i=0}^{3}\left[T_{i}, t_{i}\right] .
$$

This shows the claim.

Definition 6.23. Let $t_{0}, t_{1}, t_{2}, t_{3}: \mathfrak{C}^{\infty}(\bar{I}, \mathfrak{u}(k))$. Then we define

$$
\mathbf{t}=\mathrm{t}_{0}+i t_{1}+j t_{2}+k t_{3} \in \mathfrak{C}^{\infty}(\overline{\mathrm{I}}, \mathfrak{u}(\mathrm{k}) \otimes \mathbb{H})
$$

Combining the last two propositions, we get

Proposition 6.24. Let $\mathrm{c}=\left(\mathrm{T}_{0}, \mathrm{~T}_{1}, \mathrm{~T}_{2}, \mathrm{~T}_{3}\right) \in \mathrm{N}_{\mathrm{k}}^{(\mathrm{s})}$. Identify $\mathrm{T}_{[\mathrm{c}]} \mathcal{N}_{\mathrm{k}}^{(\mathrm{s})}$ with the orthogonal complement of the infinitesimal generators of $\mathcal{G}_{0} \frown \mathrm{N}_{k}^{(s)}$ in $\mathrm{T}_{\mathrm{c}} \mathrm{N}_{\mathrm{k}}^{(\mathrm{s})}$. This identifies $\mathrm{T}_{[\mathrm{c}]} \mathcal{N}_{\mathrm{k}}^{(\mathrm{s})}$ with quadruples $\left(t_{0}, t_{1}, t_{2}, t_{3}\right)$ where

$$
t_{i}: \bar{I} \rightarrow \mathfrak{u}(k), \quad i \in\{0,1,2,3\},
$$

that satisfy the quaternionic ordinary differential equation

$$
\begin{aligned}
\dot{\mathbf{t}}+\operatorname{ad}_{\mathrm{T}_{0}} \mathbf{t}+\operatorname{ad}_{\mathrm{T}_{1}}(\mathbf{t}(-\mathfrak{i}))+\operatorname{ad}_{\mathrm{T}_{2}}(\mathbf{t}(-\mathfrak{j}))+\operatorname{ad}_{\mathrm{T}_{3}}(\mathbf{t}(-\mathrm{k})) & =0 & & \text { for } \mathrm{N}_{\mathrm{k}}, \\
\dot{\mathbf{t}}+\operatorname{ad}_{\mathrm{T}_{0}} \mathbf{t}-\operatorname{ad}_{\mathrm{T}_{1}}(\mathfrak{i t})-\operatorname{ad}_{\mathrm{T}_{2}}(\mathfrak{j} \mathbf{t})-\operatorname{ad}_{\mathrm{T}_{3}}(\mathrm{k} \mathbf{t}) & =0 & & \text { for } \mathrm{N}_{\mathrm{k}}^{\mathrm{s}} .
\end{aligned}
$$


Proof. The above equation is just the combination of the linearized Nahm equations (6.19) and the orthogonality condition 6.27).

Corollary 6.25. There are complex structures on $\mathcal{N}_{k}$ given by left multiplication of $i, j, k$, and on $\mathcal{N}_{\mathrm{k}}^{\mathrm{s}}$ by right multiplication of $-\mathrm{i},-\mathrm{j},-\mathrm{k}$.

Proof. The equations above are invariant under the given multiplications. For the integrability see e.g. [AH88].

\subsubsection{Actions on the Moduli Space}

Definition 6.26. Define an action $\mathbb{R}^{3} \triangleleft W_{N}$ by

$$
\left(r_{1}, r_{2}, r_{3}\right) \cdot\left(T_{0}, T_{1}, T_{2}, T_{3}\right)=\left(T_{0}, T_{1}+i r_{1} \mathbb{1}, T_{2}+i r_{2} \mathbb{1}, T_{3}+i r_{3} \mathbb{1}\right) .
$$

Proposition 6.27. The action given by 6.37)

1. maps solutions of the Nahm equations to solutions of the Nahm equations,

2. respects the boundary conditions $N C_{k}$,

3. commutes with the gauge action of $\mathcal{G}$,

4. leaves $\mathrm{g}, \mathrm{\omega}_{\mathrm{I}}, \mathrm{\omega}_{\mathrm{J}}$ and $\mathrm{\omega}_{\mathrm{K}}$ invariant.

Put together, the action (6.37) induces an action $\left.\mathbb{R}^{3}\right\lrcorner \mathcal{N}_{k}^{(s)}$ which is hyperkähler.

Proof. Ad 1. The action does not change the differentials $\dot{\mathrm{T}}_{i}$ and the added terms are in the center of $\mathfrak{u}(k)$, so they also do not change the brackets $[-,-]$.

Ad 2. Satisfaction of the boundary condition at $t=1$ is equivalent to

$$
\phi_{j}=\frac{\rho_{j}}{t-1}+b_{j} ; \quad b_{j} \in \mathcal{O}(1), j \in\{1,2,3\} .
$$

But then $\phi_{j}+i r_{j} \mathbb{1}$ satisfies it with $b_{j}-i r_{j} \mathbb{1} \in \mathcal{O}(1)$.

Ad 3. For $j \in\{1,2,3\},\left(r_{1}, r_{2}, r_{3}\right) \in \mathbb{R}^{3}, g \in \mathcal{G}_{0}$ we have

$$
\text { g. }\left(r \cdot T_{j}\right)=g\left(T_{j}+i r_{j} \mathbb{1}\right) g^{-1}=g \cdot T_{j}+i r_{j} \mathbb{1}=r \cdot\left(g \cdot T_{j}\right) .
$$

Ad 4. The definition of the metric is independent of the base point $\left(T_{0}, T_{1}, T_{2}, T_{3}\right)$. Precisely, the induced action on the tangent bundle is trivial.

Definition 6.28. Define an action $\mathbf{S O}(3) \frown W_{N}$ by

$$
\text { B. }\left(T_{0}, T_{1}, T_{2}, T_{3}\right)=\left(T_{0}, \sum_{j=1}^{3} b_{1 j} T_{j}, \sum_{j=1}^{3} b_{2 j} T_{j}, \sum_{j=1}^{3} b_{3 j} T_{j}\right) \text {, }
$$

where $B=\left(b_{i j}\right)_{i, j} \in \mathbf{S O}(3)$. 
Proposition 6.29. The action given by 6.40 maps solutions of the Nahm equations to solutions of the Nahm equations.

Proof. Let $\left(T_{0}, T_{1}, T_{2}, T_{3}\right)$ be a solution of the Nahm equations. We may write the Nahm equations as

$$
0=\operatorname{Im}(\dot{\mathbf{T}})+\left[\mathrm{T}_{0}, \operatorname{Im}(\mathbf{T})\right]+\frac{1}{2} \operatorname{Im}\left((\operatorname{Im} \mathbf{T})^{2}\right) .
$$

The last term is the definition of the cross product on $\mathbb{R}^{3}$ (up to $[-,-]$, which is bilinear). Since

$$
\mathbb{R}^{3} \times \mathbb{R}^{3} \rightarrow \mathbb{R}^{3}, \quad(v, w) \mapsto v \times w .
$$

is $\mathbf{S O}(3)$-equivariant (use the definition $(v, w, v \times w) \in \mathbf{S O}(3)$ ), the Nahm equations are SO(3) equivariant,

$$
\mu_{N}(B T)=B \mu_{N}(\mathbf{T})
$$

which shows the claim.

Remark 6.30. A quick inspection shows that the action of $\mathbf{S O}(3)$ does not respect the boundary conditions. Hence we define a modified action, that does.

Definition 6.31. Define an action $\mathbf{S O}(3) \frown W_{N}$ by

$$
\text { B. }\left(T_{0}, T_{1}, T_{2}, T_{3}\right)=P^{-1} \cdot\left(T_{0}, \sum_{j=1}^{3} b_{1 j} T_{j}, \sum_{j=1}^{3} b_{2 j} T_{j}, \sum_{j=1}^{3} b_{3 j} T_{j}\right) \text {, }
$$

where $B=\left(b_{i j}\right)_{i, j} \in \mathbf{S O}(3)$ and $\mathrm{P} \in \mathbf{U}(k) \cong \operatorname{Const}(\overline{\mathrm{I}}, \mathbf{U}(k))$ is defined as follows. Denote by $\rho: \mathfrak{s u}(2) \rightarrow \mathfrak{u}(\mathrm{k})$ the irreducible representation of the boundary condition $\mathrm{NC}_{\mathrm{k}}$. This uniquely corresponds to a group homomorphism

$$
\varphi: \mathbf{S U}(2) \rightarrow \mathbf{U}(k),
$$

because $\pi_{1}(\mathbf{S U}(2))=1$. Now pick a $\tilde{\mathrm{P}} \in \mathbf{S U}(2)$ covering $\mathrm{B} \in \mathbf{S O}(3)$ with respect to the isomorphism $\mathfrak{s u}(2) \cong \mathbb{R}^{3}$ induced by mapping the canonical basis of $\mathbb{R}^{3}$ to the Pauli matrices $\sigma_{1}, \sigma_{2}, \sigma_{3}$, and let $\mathrm{P}=\varphi(\tilde{\mathrm{P}})$.

Remark 6.32. $P$ is only defined up to \pm 1 , but since the action of the gauge group factors through the center of the group, the action above is well defined.

Proposition 6.33. The modified action given by 6.44)

1. maps solutions of the Nahm equations to solutions of the Nahm equations,

2. respects the boundary conditions $N C_{\mathrm{k}}$,

3. induces a well defined action on the quotient $\mathcal{N}_{\mathrm{k}}$.

4. leaves g invariant, 
5. rotates $\mathrm{I}, \mathrm{J}, \mathrm{K}$.

Proof. Ad 1. Because the action of B preserves the Nahm equations as does the action of $\mathrm{P}$, this follows immediately.

Ad 2. Let $\varphi: \mathbf{S U}(2) \rightarrow \mathbf{U}(k)$ be the exponentiation of $\rho: \mathfrak{s u}(2) \rightarrow \mathfrak{u}(k)$. Let further $c$ denote the conjugation on both $\mathbf{S U}(2)$ and $\mathbf{U}(k)$, i.e. $c_{g}(h)=g h g^{-1}$. Then because $\varphi$ is a group homomorphism $\varphi \circ \mathbf{c}_{g}=\mathrm{c}_{\varphi(g)} \circ \varphi$ for all $\mathrm{g} \in \mathbf{S U}(2)$. Differentiating this equation at $\mathbb{1} \in \mathbf{S U}(2)$ gives

$$
\begin{aligned}
\rho \circ \operatorname{Ad}_{\mathrm{g}} & =\mathrm{D}_{\mathbb{1}} \varphi \circ \mathrm{D}_{\mathbb{1}} \mathrm{c}_{\mathrm{g}}=\mathrm{D}_{\mathbb{1}}\left(\varphi \circ \mathrm{c}_{\mathrm{g}}\right) \\
& \stackrel{!}{=} \mathrm{D}_{\mathbb{1}}\left(\mathrm{c}_{\varphi(\mathrm{g})} \circ \varphi\right)=\mathrm{D}_{\mathbb{1}} \mathrm{c}_{\varphi(\mathrm{g})} \circ \mathrm{D}_{\mathbb{1}} \varphi=\mathrm{Ad}_{\varphi(g)} \circ \rho .
\end{aligned}
$$

Now let $\tilde{P}$ be the lift of $B \in \mathbf{S O}(3)$ to $\mathbf{S U}(2)$, then this calculation implies that $\rho \circ \operatorname{Ad}_{\tilde{\mathrm{P}}-1}=\mathrm{Ad}_{\mathrm{P}-1} \circ \rho$.

The action on the boundary condition is given by mapping

$$
\mathrm{T}_{\mathrm{i}}=\frac{\rho_{\mathrm{i}}}{\mathrm{t}-1}+\mathcal{O}(1)
$$

to

$$
\tilde{T}_{i}=\operatorname{Ad}_{P^{-1}}\left(\sum_{j=1}^{3} b_{i j} T_{j}\right)=\frac{\operatorname{Ad}_{p^{-1}}\left(\sum_{j=1}^{3} b_{i j} \rho_{j}\right)}{t-1}+\mathcal{O}(1) .
$$

Because $\rho$ is linear and the calculation above holds, we have

$$
\operatorname{Ad}_{\mathrm{P}^{-1}}\left(\sum_{j=1}^{3} b_{i j} \rho_{j}\right)=\rho \circ \operatorname{Ad}_{\tilde{\mathrm{P}}-1}\left(\sum_{j=1}^{3} b_{i j} \sigma_{j}\right)=\rho\left(\sigma_{j}\right)=\rho_{j} .
$$

Ad 3. Let $B \in \mathbf{S O}(3)$ be a matrix and $P \in \mathbf{U}(k)$ a choice as in the definition of the action. We have to show that the induced action is well defined on the equivalence classes $\mathcal{N}_{k}$. Hence let $\left[\left(T_{0}, \vec{T}\right)\right]$ be an equivalence class of solutions to the Nahm equations with boundary $\mathrm{NC}_{k}, k \in \mathbb{N}$, and let $\mathrm{g} \in \mathcal{G}_{0}$ be an admissible gauge. We have to show that

$$
\left[(\mathrm{P}, \mathrm{B}) \cdot\left(\mathrm{g} \cdot\left(\mathrm{T}_{0}, \overrightarrow{\mathrm{T}}\right)\right)\right]=\left[(\mathrm{P}, \mathrm{B}) \cdot\left(\mathrm{T}_{0}, \overrightarrow{\mathrm{T}}\right)\right] .
$$

For this define $\mathrm{g}^{\prime}=\operatorname{Ad}_{\mathrm{p}} \mathrm{g}^{-1} \in \mathcal{G}_{0}$ and recall that the action of $\mathrm{g}$ commutes with the action of $\mathrm{B} \in \mathbf{S O}(3)$, hence

$$
g^{\prime} \cdot(P, B) \cdot\left(g \cdot\left(T_{0}, \vec{T}\right)\right)=g^{\prime} \cdot P \cdot g \cdot\left(T_{0}, B \vec{T}\right)=P \cdot\left(T_{0}, B \vec{T}\right)=(P, B) \cdot\left(T_{0}, \vec{T}\right) .
$$

Ad 4. This is immediately clear because the scalar product on $\mathbb{R}^{4} \cong \mathbb{H}$ is $\mathbf{S O}(3) \hookrightarrow$ SO(4) invariant.

Ad 5. This is obvious as the action of $B \in \mathbf{S O}(3)$ is simply the rotating action on $\mathbb{H}$ and the action of $\mathrm{P} \in \mathfrak{C}^{\infty}(\mathrm{I}, \mathrm{U}(\mathrm{k}))$ leaves $\mathrm{I}, \mathrm{J}$ and $\mathrm{K}$ invariant. 


\subsubsection{As a Hyperkähler Reduction}

The Nahm equations can also be described (formally) as a hyperkähler reduction. The formal part about it is that it involves infinite dimensional manifolds, and so some analytic work has to be done to make it precise. For these details see e.g. [Bieo8].

Proposition 6.34. The action of $\mathcal{G}_{\mathrm{N}} \triangleleft \mathrm{N}_{\mathrm{k}}^{(\mathrm{s})}$ is trihamiltonian and the corresponding moment map is given by $\pm \mu_{N}^{(s)}: \mathrm{F}_{N} \rightarrow \mathrm{U}_{\mathrm{N}}$ (again, the upper sign is for $\mathrm{N}_{\mathrm{k}}$, the lower for $\mathrm{N}_{\mathrm{k}}^{\mathrm{s}}$ ). Thus $\mathcal{N}_{\mathrm{k}}^{(\mathrm{s})}$ is a formal hyperkähler reduction of the affine space $\mathrm{F}_{\mathrm{N}}$.

Proof. We already know that the action of the gauge group is hyperkähler, so the only remaining thing to show is that

$$
\mathrm{d} \mu_{\mathrm{A}}^{(s)}(-)(\xi)=\omega_{A}^{(s)}\left(\mathrm{K}^{\xi},-\right) \quad \forall \xi \in \operatorname{Lie}(\mathcal{G}), \forall \mathrm{A} \in\{\mathrm{I}, \mathrm{J}, \mathrm{K}\} .
$$

We will show this as an example for $d \mu_{\mathrm{I}}^{(s)}$. Let $X=\left(s_{0}, s_{1}, s_{2}, s_{3}\right)$ be a tangent vector at $\left(T_{0}, T_{1}, T_{2}, T_{3}\right) \in N_{k}^{(s)}$, then

$$
\mathrm{d} \mu_{\mathrm{I}}^{(s)}(X)=\dot{s}_{1}+\left[s_{0}, \mathrm{~T}_{1}\right]+\left[\mathrm{T}_{0}, s_{1}\right] \pm\left[\mathrm{s}_{2}, \mathrm{~T}_{3}\right] \pm\left[\mathrm{T}_{2}, \mathrm{~s}_{3}\right]
$$

and

$$
\begin{aligned}
g\left(\mathrm{I}^{(s)} \mathrm{K}^{\xi}, \mathrm{X}\right) & =\int_{\mathrm{I}}\left\langle\mp\left[\xi, \mathrm{T}_{1}\right], \mathrm{s}_{0}\right\rangle+\left\langle\mp \mathrm{d}_{\mathrm{T}_{0}} \xi, \mathrm{s}_{1}\right\rangle+\left\langle-\left[\xi, \mathrm{T}_{3}\right], \mathrm{s}_{2}\right\rangle+\left\langle\left[\xi, \mathrm{T}_{2}\right], \mathrm{s}_{3}\right\rangle \mathrm{dt} \\
& =\int_{\mathrm{I}}\left\langle\xi, \pm \mathrm{d}_{\mathrm{T}_{0}} \mathrm{~s}_{1} \pm\left[\mathrm{s}_{0}, \mathrm{~T}_{1}\right]+\left[\mathrm{s}_{2}, \mathrm{~T}_{3}\right]+\left[\mathrm{T}_{2}, \mathrm{~s}_{3}\right]\right\rangle \mathrm{dt} \\
& =\int_{\mathrm{I}}\left\langle\xi, \pm \mathrm{d} \mu_{\mathrm{I}}^{\mathrm{s}}(\mathrm{X})\right\rangle \mathrm{dt}= \pm \mathrm{d} \mu_{\mathrm{I}}^{\mathrm{s}}(\mathrm{X})(\xi) .
\end{aligned}
$$

Remark 6.35. The canonical connection on $\mu_{N}^{-1}(0) \rightarrow \mathcal{N}_{k}$ can be described as follows. Since the action of $\mathcal{G}_{0}$ is free, for every point $\left(T_{0}, T_{1}, T_{2}, T_{3}\right) \in \mu_{N}^{-1}(0)$ we have a pulled back metric

$$
\langle\xi, \eta\rangle_{\left(T_{i}\right)}=g\left(K_{\left(T_{i}\right)}^{\xi}, K_{\left(T_{i}\right)}^{\eta}\right)
$$

on $\operatorname{Lie}\left(\mathcal{G}_{0}\right)$. Let $S_{\left(T_{i}\right)}$ denote the self-adjoint operator between the fixed scalar product on $\operatorname{Lie}\left(\mathcal{G}_{0}\right)$ and this induced one, i.e.

$$
\left\langle S_{\left(T_{i}\right)} \xi, \eta\right\rangle_{s t}=\langle\xi, \eta\rangle_{\left(T_{i}\right)}
$$

for all $\xi, \eta \in \operatorname{Lie}\left(\mathcal{G}_{0}\right)$ and $\left(T_{i}\right) \in \mu^{-1}(0)$. We see that the inverse of $S_{\left(T_{i}\right)}$ describes the connection form $A$ of the canonical connection,

$$
\left\langle A_{\left(T_{i}\right)}\left(t_{i}\right), \eta\right\rangle_{s t}=g\left(\left(t_{i}\right), K^{S_{\left(T_{i}\right)}}{ }^{-1} \eta\right),
$$

for all $\left(T_{i}\right) \in \mu_{N}^{-1}(0),\left(t_{i}\right) \in T_{\left(T_{i}\right)} \mu_{N}^{-1}(0)$ and $\eta \in \operatorname{Lie}\left(\mathcal{G}_{0}\right)$. 
Proposition 6.36. The operator

$$
S_{\left(T_{i}\right)}=-d_{T_{0}}^{2}-\sum_{i=1}^{4} \operatorname{ad}_{T_{i}}^{2}
$$

where

$$
\mathrm{d}_{\mathrm{T}_{0}}=\frac{\mathrm{d}}{\mathrm{dt}}+\mathrm{ad}_{\mathrm{T}_{0}}
$$

satisfies

$$
\left\langle S_{\left(T_{i}\right)}, \eta\right\rangle_{s t}=\langle\xi, \eta\rangle_{\left(T_{i}\right)}
$$

for all $\xi, \eta \in \operatorname{Lie}\left(\mathcal{G}_{0}\right)$ and $\left(\mathrm{T}_{i}\right) \in \mu^{-1}(0)$. Furthermore it is $\mathcal{G}$-equivariant, i.e.

$$
\operatorname{Ad}_{g^{-1}} \circ S_{g\left(T_{i}\right)} \xi=S_{\left(T_{i}\right)} \operatorname{Ad}_{g^{-1}} \xi
$$

for all $\xi \in \operatorname{Lie}(\mathcal{G})$. If we restrict to boundary conditions that makes $S_{\left(T_{i}\right)}$ a well defined endomorphism, then the one form A of the canonical connection is given by

$$
\left\langle A_{\left(T_{i}\right)}\left(t_{i}\right), \eta\right\rangle_{s t}=g\left(\left(t_{i}\right), K^{S_{\left(T_{i}\right)}}{ }^{-1} \eta\right),
$$

for all $\left(\mathrm{T}_{i}\right) \in \mu_{\mathrm{N}}^{-1}(0),\left(\mathrm{t}_{\mathrm{i}}\right) \in \mathrm{T}_{\left(\mathrm{T}_{i}\right)} \mu_{\mathrm{N}}^{-1}(0)$ and $\eta \in \operatorname{Lie}\left(\mathcal{G}_{0}\right)$.

Proof. For the boundary conditions and the fact that $S_{\left(T_{i}\right)}$ is invertible, see [Bieo8]. $S_{\left(T_{i}\right)}$ satisfies the equation 6.63 because

$$
\begin{aligned}
\langle\xi, \eta\rangle_{\left(T_{i}\right)} & =g\left(K_{\left(T_{i}\right)}^{\xi}, K_{\left(T_{i}\right)}^{\eta}\right) \\
& =\int_{I}\left\langle d_{T_{0}} \xi, d_{T_{0}} \eta\right\rangle+\sum_{j=0}^{4}\left\langle\left[T_{j}, \xi\right],\left[T_{j}, \eta\right]\right\rangle d t \\
& =\int_{I}\left\langle\left(-\frac{d^{2}}{d t^{2}}-a d_{\dot{T}_{0}}-2 a d_{T_{0}} \circ \frac{d}{d t}-\sum_{i=0}^{4} a d_{T_{i}}^{2}\right) \xi, \eta\right\rangle d t .
\end{aligned}
$$

The $\mathcal{G}$-equivariance is a cumbersome but straight-forward calculation.

\subsection{Bogomolny Equations}

\subsubsection{The Equations}

The Bogomolny equations can be studied for arbitrary 3-manifolds and principal bundles on them. We are however only interested in the Bogomolny equations on $\mathbb{R}^{3}$ here. Thus any bundle can then be trivialized and the connection be interpreted as a form on the base. The choice of trivialization is not important after passing to gauge equivalence classes; a different choice of trivialization is given by gauging the Bogomolny datum of the first trivialization. 
Definition 6.37. A Bogomolny datum is a pair $(\phi, A) \in \mathfrak{C}^{\infty}\left(\mathbb{R}^{3}, \mathfrak{g}\right) \times \Omega^{1}\left(\mathbb{R}^{3}, \mathfrak{g}\right)$ for some Lie Algebra $\mathfrak{g}$.

Remark 6.38. Sometimes it will be convenient to combine these to

$$
\mathbf{A}: \mathbb{R}^{3} \rightarrow \mathbb{H} \otimes \mathfrak{g},
$$

where $\operatorname{Re}(\mathbf{A})=\phi$ and we identified $\Omega^{1}\left(\mathbb{R}^{3}\right)$ with $\mathfrak{C}^{\infty}\left(\mathbb{R}^{3}\right)^{3}$ via evaluation on the vector fields induced by the canonical basis of $\mathbb{R}^{3}$.

Notation 6.39. We introduce the following notation,

$$
W_{\mathrm{B}}=\mathfrak{C}^{\infty}\left(\mathbb{R}^{3}, \mathbb{H} \otimes \mathfrak{g}\right) \quad \text { and } \quad \mathrm{U}_{\mathrm{B}}=\mathfrak{C}^{\infty}\left(\mathbb{R}^{3}, \operatorname{Im}(\mathbb{H}) \otimes \mathfrak{g}^{\vee}\right) .
$$

Definition 6.40. A Bogomolny datum satisfies the Bogomolny Equations if

$$
\mathrm{F}^{\mathrm{A}}=\star \mathrm{d}_{\mathrm{A}} \phi
$$

Define the map

$$
\mu_{\mathrm{B}}: \mathrm{W}_{\mathrm{B}} \rightarrow \mathrm{U}_{\mathrm{B}}, \quad(\phi, \mathrm{A}) \mapsto \mathrm{F}^{\mathrm{A}}-\star \mathrm{d}_{\mathrm{A}} \phi .
$$

Remark 6.41. From the reduction theory, we know that $A$ is a connection and $\phi$ is a sections of the adjoint bundle. This allows us to define an action of the gauge group $\mathcal{G}_{\mathrm{B}}=\mathfrak{C}^{\infty}\left(\mathbb{R}^{3}, \mathrm{G}\right)$ (from now on just $\mathcal{G}$ in this section) on the Bogomolny data by

$$
g \cdot A=\operatorname{Ad}_{g} A-g^{*} \omega_{G}, \quad g . \phi=\operatorname{Ad}_{g} \phi .
$$

For matrix groups this is given by $g . A=\mathrm{Ad}_{\mathrm{g}} \circ \mathrm{A}-\dot{\mathrm{g}} \mathrm{g}^{-1}$.

By standard formulas for the gauge action we have g. $F^{A}=\operatorname{Ad}_{g} F^{A}$ and $d_{g \cdot A}(g \cdot \phi)=$ $\operatorname{Ad}_{g} d_{A} \phi$, so the Bogomolny equations are $\mathcal{G}$-equivariant,

$$
\mu_{\mathrm{B}}(\mathrm{g} \cdot \mathbf{A})=\operatorname{Ad}_{\mathrm{g}} \circ \mu_{\mathrm{B}}(\mathbf{A}),
$$

hence the preimage of 0 is invariant; so the gauge group acts on solutions of the Bogomolny equations.

\subsubsection{Boundary Conditions}

From now on fix $G=\mathbf{S U}(2)$, for $k \in \mathbb{N}$ and let $r=\sqrt{x^{2}+y^{2}+z^{2}}$ denote the radial euclidean distance on $\mathbb{R}^{3}$.

Definition 6.42. We say a pair $(\phi, A)$ satisfies the boundary conditions for $k \in \mathbb{N}$ (satisfies $\mathrm{BC}_{\mathrm{k}}$ ) if for $\mathrm{r} \rightarrow \infty$ we have the following asymptotics

$$
\begin{aligned}
\|\phi\| & =1-\frac{k}{2 r}+\mathcal{O}\left(r^{-2}\right), \\
d\|\phi\| & =\mathcal{O}\left(r^{-2}\right), \\
\left\|d_{A} \phi\right\| & =\mathcal{O}\left(r^{-2}\right) .
\end{aligned}
$$


Remark 6.43. 1. Taubes has shown (unpublished) that it suffices to require $\|\phi\| \rightarrow 1$ as $r \rightarrow \infty$ if $(\phi, A)$ satisfy the Bogomolny equations using techniques from [JT80].

2. $k \in \mathbb{N}$ is implied by the other conditions, if we let $k \in \mathfrak{C}^{\infty}\left(\mathbb{R}^{3}\right)$. The second condition implies that $k$ is constant, and there are multiple ways to see that $k$ is an integer, the most descriptive are

- Since $\|\phi\| \rightarrow 1$, there is a $R>0$ such that $\phi$ has no zeroes outside of the ball of radius $R$ centered at the origin. Then $x \rightarrow \frac{\phi}{\|\phi\|}$ when restricted to sphere of radius bigger than $R$ gives a smooth map from $S^{2} \rightarrow S^{2}, k$ is just the negative of the degree of this map.

- By gauging the vector field $\phi$ on $\mathbb{R}^{3}$ to an analytic solution (this can be achieved locally, see [JT80], we see that $\phi$ has isolated zeroes. These are invariant under gauging. Using the theorem of Poincaré-Hopf, we can calculate the Euler characteristic of $\mathbb{R}^{3}$ without the complement of a large ball by counting multiplicities of the zeroes of the vector field $\phi$. This is related to $k \in \mathbb{N}$ by the winding of $\phi$ at infinity (see [AFG75]).

- On the complement $U$ of the large ball we can interpret $x \rightarrow \frac{\phi}{\|\phi\|}$ as a reduction of the principal $\mathbf{S U}(2)$ bundle, i.e. a $\mathbf{S U}(2)$-equivariant map

$$
\mathrm{P} \mid \mathrm{u} \rightarrow \mathrm{SU}(2) / \mathbf{U}(1) \cong \mathrm{S}^{2} .
$$

Hence $\phi$ defines an $\mathbf{U}(1)$-bundle $Q$ which is characterized by $c_{1}(Q)=k$. The adjoint bundle of the original (trivial) bundle $P$ then splits into two lines bundles of degree $k$ and $-k$.

3. The value of $\|\phi\|$ when $r \rightarrow \infty$ is arbitrary. Physically speaking it is the mass of the monopole, but the moduli spaces are the same for every choice of mass.

While the gauge group is leaving the boundary conditions $B C_{k}$ invariant, we still introduce a partial framing at infinity.

Definition 6.44. Define the reduced gauge group to be

$$
\mathcal{G}_{0}=\left\{g \in \mathcal{G}_{\mathrm{B}} \mid \lim _{x \rightarrow \infty} g(x, 0,0)=1\right\}
$$

Remark 6.45. As mentioned, any $\mathbf{S U}(2)$-monopole reduces to a $\mathbf{U}(1)$-monopole on a sphere at infinity (i.e. on the complement of a ball large enough). The induced data (the projected connection and induced Higgs field) satisfy the $\mathbf{U}(1)$-monopole equation $(\mathrm{d} A=\star d \phi)$. The equivalence class of such reductions to the fixed $\mathbf{U}(1)$-monopole has a $\mathbf{U}(1)$-action (acting by constant gauges). However, since monopole connections are irreducible, it only fixes the $\mathbf{S U}(2)$-connection if it is in the center of $\mathbf{S U}(2)$, hence only \pm 1 extend to monopole morphisms. 
As always with framing, instead of framing the gauges, we can also add a fiber at one point. For this picture we can fix a reduction to a $\mathbf{U}(1)$-monopole as above. The resulting space then is a $\mathbf{U}(1) /\{ \pm 1\}$ bundle over the former quotient. This picture is more cumbersome to denote but has more geometrical insights. In particular it allows us to define a rotating action. To see how to switch between these pictures, see [AH88].

\subsubsection{The Moduli Space}

Notation 6.46. Denote by $F_{B}$ the subspace of $W_{B}$ that satisfies the boundary conditions $\mathrm{BC}_{\mathrm{k}}$ for some $k \in \mathbb{N}$ ( $\mathrm{F}_{\mathrm{B}}^{\mathrm{k}}$ if we want to fix the $k \in \mathbb{N}$ ).

Definition 6.47. Fix $k \in \mathbb{N}$. With $M_{k}$ we denote the space of solutions to the Bogomolny equations for $G=\mathbf{S U}(2)$, subject to the boundary condition $B C_{k}$. The framed gauge group $\mathcal{G}_{0}$ acts freely on this space, and the quotient is a $4 \mathrm{k}$ dimensional hyperkähler manifold [AH88] denoted by

$$
\mathcal{M}_{\mathrm{k}}=\mathrm{M}_{\mathrm{k}} / \mathcal{G}_{0} \text {. }
$$

Remark 6.48. Just as in the Nahm case, we can define the self dual version of the Bogomolny equations. The reduction theory shows that these are given by

$$
\mathrm{F}^{\mathrm{A}}+\star \mathrm{d}_{\mathrm{A}} \phi=0,
$$

and similarly to the Nahm case they correspond to a change of orientation of $\mathbb{R}^{4}$. We define everything else similarly to the regular Bogomolny equations and denote the space by $\mathcal{M}_{k}^{s}=M_{k}^{s} / \mathcal{G}_{0}$. Note that $M_{k}^{s} \cong M_{k}$ by mapping $(\phi, A) \rightarrow(-\phi, A)$.

Lemma 6.49. $F_{B}$ is an affine space over

$$
\mathrm{V}_{\mathrm{B}}=\mathbb{H} \otimes\left\{f \in \mathrm{L}^{2}\left(\mathbb{R}^{3}, \mathfrak{g}\right) \mid \mathrm{f} \in \mathcal{O}\left(\mathrm{r}^{-2}\right)\right\},
$$

where we understand all $\mathrm{L}^{2}$ as smooth square integrable functions.

Proof. Being in $\mathcal{O}\left(\mathrm{r}^{-2}\right)$ ensures that the boundary condition of $\phi$ is not changed.

Note that we could let $M_{k}^{(s)}$ be an affine space over the larger vector space, where we do not restrict to (smooth) $\mathrm{L}^{2}$ functions. However, Taubes has shown [JT80] that it suffices to take $\mathrm{L}^{2}$ sections to describe the tangent space. We sometimes interpret $V_{B}$ as given in the Lemma, other times it is more convenient to think about $\mathrm{V}_{\mathrm{B}}=\mathrm{L}^{2}\left(\mathbb{R}^{3}, \mathfrak{s u}(2)\right) \oplus \Omega_{\mathrm{L}^{2}}^{1}\left(\mathbb{R}^{3}, \mathfrak{s u}(2)\right)$, with the correct boundary behavior, as in the next proposition.

Proposition 6.50. Let $\mathbf{A} \in \mathrm{M}_{\mathrm{k}}^{(\mathrm{s})}$. Then the space $\mathrm{T}_{\mathbf{A}} \mathrm{M}_{\mathrm{k}}^{(\mathrm{s})}$ consists of pairs $(\psi, \mathrm{a}) \in \mathrm{V}_{\mathrm{B}}$ that satisfy the linearized (self dual) Bogomolny equations

$$
\star d_{A} a \pm d_{A} \psi \pm[a, \phi]=0 .
$$

Again, the upper sign is for the case of $\mathrm{M}_{\mathrm{k}}$, the lower sign for $\mathrm{M}_{\mathrm{k}}^{(\mathrm{s})}$. 
Proof. Note that

$$
d_{A+\epsilon a}(\phi+\epsilon \psi)=d_{A} \phi+\epsilon\left(d_{A} \psi+[a, \phi]\right)+\mathcal{O}\left(\epsilon^{2}\right),
$$

so that the linearization $\star F^{A} \pm d_{A} \phi$ is given by the term of the proposition.

Definition 6.51. Define the usual metric for bundle valued forms on $M_{k}^{(s)}$, i.e.

$$
\langle\mathbf{A}, \mathbf{B}\rangle=\int_{\mathbb{R}^{3}} \sum_{i=0}^{3}\left\langle\mathbf{A}_{i}, \mathbf{B}_{i}\right\rangle \operatorname{vol}_{\mathbb{R}^{3}}
$$

where $\mathbf{A}$ and $\mathbf{B}$ are tangent vectors as in Proposition $(6.50)$ and $\langle-,-\rangle$ on $\mathfrak{s u}(2)$ is given by $-\frac{1}{2} \operatorname{tr}(\mathrm{ts})$.

Lemma 6.52. The induced action of the gauge group $\mathcal{G}$ on the tangent space $\mathrm{TM}_{\mathrm{k}}^{(\mathrm{s})}$ is given by

$$
\text { g. }(\psi, a)=\left(\operatorname{Ad}_{g} \psi, \operatorname{Ad}_{g} a\right) \text {. }
$$

Proof. See Lemma 6.19.

Proposition 6.53. The metric is invariant under the gauge action $9_{0} \triangleleft \mathrm{M}_{\mathrm{k}}^{(s)}$.

Proof. This follows immediately since the Killing form is Ad-invariant.

Lemma 6.54. The infinitesimal action of the Lie Algebra of $\mathcal{G}_{0}$,

$$
\operatorname{Lie}\left(\mathcal{G}_{0}\right)=\left\{\xi \in \Gamma\left(\mathbb{R}^{3}, \operatorname{Ad}(\mathrm{P})\right) \mid\|\xi\| \in \mathcal{O}\left(\mathrm{r}^{-1}\right)\right\}
$$

on $\mathrm{M}_{\mathrm{k}}^{(\mathrm{s})}$ is given by

$$
\mathrm{K}^{\xi}=\left([\xi, \phi],-\mathrm{d}_{\mathrm{A}} \xi\right) .
$$

Proof. See the proof of Lemma 6.21 .

Proposition 6.55. A tangent vector $(\psi, \mathrm{a})$ to $\mathrm{c}=(\phi, A) \in \mathrm{M}_{\mathrm{k}}^{(s)}$ is orthogonal to the compactly supported infinitesimal generators of $\mathcal{G}_{0} \triangleleft \mathrm{M}_{\mathrm{k}}^{(s)}$ with respect to the metric (6.20) if and only if

$$
\star \mathrm{d}_{\mathrm{A}} \star \mathrm{a}+[\phi, \psi]=0
$$

Proof. We want $g\left(K^{\xi},(\psi, A)\right)=0$, hence

$$
0=\int_{\mathbb{R}^{3}}\left\langle-\mathrm{d}_{\mathcal{A}} \xi, \mathrm{a}\right\rangle+\langle[\xi, \phi], \psi\rangle \operatorname{vol}_{\mathbb{R}^{3}},
$$


and

$$
\begin{aligned}
\int_{\mathbb{R}^{3}}\left\langle d_{A} \xi, a\right\rangle & =\int \sum_{i}\left\langle d_{A} \xi\left(\partial_{i}\right), a\left(\partial_{i}\right)\right\rangle \operatorname{vol}_{\mathbb{R}^{3}} \\
& =\int \sum_{i}\left\langle\partial_{i} \xi, a_{i}\right\rangle+\left\langle\left[A_{i}, \xi\right], a_{i}\right\rangle \operatorname{vol}_{\mathbb{R}^{3}} \\
& =\int \operatorname{grad} \xi \cdot a+\sum_{i}\left\langle\left[A_{i}, \xi\right], a_{i}\right\rangle \operatorname{vol}_{\mathbb{R}^{3}} \\
& =\int \operatorname{div}(\xi a)-\xi \operatorname{div} a-\sum_{i}\left\langle\xi,\left[A_{i}, a_{i}\right]\right\rangle \operatorname{vol}_{\mathbb{R}^{3}},
\end{aligned}
$$

where $a_{i}=a\left(\partial_{i}\right)$ and we used the vector calculus identity $\operatorname{grad} \xi \cdot a+\xi \operatorname{div} a=\operatorname{div}(\xi a)$, and by the theorem of Stokes, $\int \operatorname{div}(\xi a) \operatorname{vol}_{\mathbb{R}^{3}}$ vanishes (we assume $\xi$ has compact support here). But then

$$
g\left(K^{\xi},(\psi, A)\right)=\int_{\mathbb{R}^{3}}\left\langle\operatorname{div} a+\sum_{i}\left[A_{i}, a_{i}\right]+[\phi, \psi], \xi\right\rangle \operatorname{vol}_{\mathbb{R}^{3}},
$$

which together with $\star d_{A} \star a=\operatorname{div} a+\sum_{i}\left[A_{i}, a_{i}\right]$ shows the claim.

Proposition 6.56. Let $\mathrm{c}=(\phi, \mathrm{A}) \in \mathrm{M}_{\mathrm{k}}^{\mathrm{s}}$. Identify $\mathrm{T}_{[\mathrm{c}]} \mathcal{M}_{\mathrm{k}}^{(\mathrm{s})}$ with the orthogonal complement of the infinitesimal generators of $\mathcal{G}_{0} \triangleleft \mathrm{M}_{\mathrm{k}}^{(\mathrm{s})}$ in $\mathrm{T}_{\mathrm{c}} \mathrm{M}_{\mathrm{k}}^{(\mathrm{s})}$. This identifies $\mathrm{T}_{[\mathrm{c}]} \mathcal{M}_{\mathrm{k}}^{(s)}$ with pairs $\mathbf{a}=(\psi, \mathrm{a}) \in \mathrm{V}_{\mathrm{B}}$ that satisfy the quaternionic ordinary differential equation

$$
\begin{array}{rlr}
{[\phi, \mathbf{a}]+\nabla_{1}^{\mathrm{A}}(\mathbf{a}(-\mathrm{i}))+\nabla_{2}^{\mathrm{A}}(\mathbf{a}(-\mathrm{j}))+\nabla_{3}^{\mathrm{A}}(\mathbf{a}(-\mathrm{k}))=0} & \text { for } \mathrm{M}_{\mathrm{k}}, \\
{[\phi, \mathbf{a}]-\nabla_{1}^{\mathrm{A}}(\mathbf{i} \mathbf{A})-\nabla_{2}^{\mathrm{A}}(\mathbf{j} \mathbf{a})-\nabla_{3}^{\mathrm{A}}(\mathrm{kA})=0} & \text { for } M_{\mathrm{k}}^{\mathrm{s}} .
\end{array}
$$

Proof. We see that the system

$$
\begin{aligned}
\star d_{A} \star a+[\phi, \psi] & =0 \\
\star d_{A} a \mp d_{A} \psi \mp[a, \phi] & =0
\end{aligned}
$$

is in coordinates given by

$$
\begin{aligned}
& \nabla_{1}^{A} a_{1}+\nabla_{2}^{A} a_{2}+\nabla_{3}^{A} a_{3}+[\phi, \psi]=0 \\
& \nabla_{2}^{A} a_{3}-\nabla_{3}^{A} a_{2} \mp \nabla_{1}^{A} \psi \pm\left[\phi, a_{1}\right]=0 \\
& \nabla_{3}^{A} a_{1}-\nabla_{1}^{A} a_{3} \mp \nabla_{2}^{A} \psi \pm\left[\phi, a_{2}\right]=0 \\
& \nabla_{1}^{A} a_{2}-\nabla_{2}^{A} a_{1} \mp \nabla_{3}^{A} \psi \pm\left[\phi, a_{3}\right]=0 .
\end{aligned}
$$

Sorting this we get

$$
\nabla_{1}^{A}\left(\begin{array}{c}
a_{1} \\
\mp \psi \\
-a_{3} \\
a_{2}
\end{array}\right)+\nabla_{2}^{A}\left(\begin{array}{c}
a_{2} \\
a_{3} \\
\mp \psi \\
-a_{1}
\end{array}\right)+\nabla_{3}^{A}\left(\begin{array}{c}
a_{3} \\
-a_{2} \\
a_{1} \\
\mp \psi
\end{array}\right)+\left[\phi,\left(\begin{array}{c}
\psi \\
\pm a_{1} \\
\pm a_{2} \\
\pm a_{3}
\end{array}\right)\right]=0,
$$

which shows the claim (for $M_{k}^{s}$ multiply the last three equations by -1). 
Corollary 6.57. There are complex structures on $\mathcal{M}_{k}$ given by left multiplication of $i, j, k$ and on $\mathcal{M}_{\mathrm{k}}^{\mathrm{s}}$ given by right multiplication of $-i,-j,-k$.

Proof. The equations above are invariant under these multiplications. For the integrability see e.g. [AH88].

\subsubsection{Actions on the Moduli Space}

Definition 6.58. Define an action $\left.\mathbb{R}^{3}\right\lrcorner \mu_{B}{ }^{-1}(0)$ by pulling back the function $\psi$ and form $A$ along the translation by $r \in \mathbb{R}^{3}$.

Proposition 6.59. The action given by Definition 6.58

1. maps solutions of the Bogomolny equations to solutions of the Nahm equations,

2. respects the boundary conditions $B C_{k}$,

3. commutes with the gauge action of $\mathcal{G}$,

4. leaves $\mathrm{g}, \mathrm{\omega}_{\mathrm{I}}, \omega_{\mathrm{J}}$ and $\omega_{\mathrm{K}}$ invariant.

Put together, the action of Definition 6.58 induces an action $\left.\mathbb{R}^{3}\right\lrcorner \mathcal{M}_{\mathrm{k}}^{(\mathrm{s})}$ which is hyperkähler. Proof. All these follow immediately.

Proposition 6.60. The action $\mathrm{SU}(2) \rightarrow \mathrm{SO}(3) \triangleleft \mathbb{R}^{3}$ induces a rotating (left) action on $\mathcal{M}_{\mathrm{k}}^{(s)}$. Proof. Map $\hat{B} \in \mathbf{S U}(2)$ to $B=\left(b_{i j}\right)_{i, j} \in \mathbf{S O}(3)$ and let it act as denoted. If we write $A=\sum_{i=1}^{3} A_{i} d x^{i}$, then the transformation of the connection becomes

$$
\begin{aligned}
\text { B. } A & =\sum_{i=1}^{3} A_{i} \circ B^{-1} d\left(x^{i} \circ B^{-1}\right)=\sum_{i=1}^{3} A_{i} \circ B^{-1} d\left(x_{i} \circ B^{t}\right) \\
& =\sum_{i, j=1}^{3} A_{i} \circ B^{-1} d x_{j} b_{i j}^{t}=\sum_{i, j=1}^{3} A_{i} \circ B^{-1} d x_{j} b_{j i}
\end{aligned}
$$

so that

$$
A_{k} \mapsto \sum_{i=1}^{3} b_{k i} A_{i} \circ B^{-1}
$$

under the action. The action on $\varphi$ is simply given by pull back, B. $\phi=\phi \circ \mathrm{B}^{-1}$. The chain rule implies that

$$
\partial_{l}\left(B . A_{k}\right)=\sum_{i=1}^{3} b_{l i} \partial_{l} A_{k} \circ B^{-1} \quad \text { and } \quad \partial_{l}(B . \phi)=\sum_{i=1}^{3} b_{l i} \partial_{i} \phi \circ B^{-1} .
$$


This shows that the equations are equivariant under this action. It follows immediately that the boundary conditions are satisfied (here it is necessary to use the geometrical boundary description). It is also easy to see that the action commutes with the gauge action and leaves $g$ invariant (because the matrix is in $\mathbf{S O}(3)$ ). Finally, it induces the usual rotating action on the flat quaternionic space $V_{B}$.

\subsubsection{As a Hyperkähler Reduction}

The Bogomolny equations can also be described (formally) as a hyperkähler reduction. The formal part is similar to the Nahm equations.

Proposition 6.61. The action of $\mathcal{G}_{\mathrm{B}} \triangleleft \mathrm{M}_{\mathrm{k}}^{(\mathrm{s})}$ is trihamiltonian and the corresponding moment map is given by $\mu_{\mathrm{B}}^{(\mathrm{s})}: \mathrm{F}_{\mathrm{B}} \rightarrow \mathrm{U}_{\mathrm{B}}$. Thus $\mathcal{M}_{\mathrm{k}}^{(\mathrm{s})}$ is a formal hyperkähler reduction of $\mathrm{F}_{\mathrm{B}}$.

Proof. We already know that the action of the gauge group is hyperkähler, so the only remaining thing to show is that

$$
\left\langle\xi, \mathrm{d} \mu_{\mathrm{A}}^{(s)}(-)\right\rangle=\omega_{\mathrm{A}}^{(s)}\left(\mathrm{K}^{\xi},-\right) \quad \forall \xi \in \operatorname{Lie}(\mathcal{G}), \forall A \in\{\mathrm{I}, \mathrm{J}, \mathrm{K}\} .
$$

We will show this as an example for $d \mu_{\mathrm{I}}^{(s)}$. Let $X=\left(s_{1}, s_{2}, s_{3}, s_{4}\right)$ be a tangent vector at $(\phi, A) \in M_{k}^{(s)}$ and $\xi$ compactly supported, then

$$
\mathrm{d} \mu_{\mathrm{I}}^{(\mathrm{s})}(\mathrm{X})=\nabla_{2}^{\mathrm{A}} \mathrm{s}_{3}-\nabla_{3}^{\mathrm{A}} \mathrm{s}_{2} \mp \nabla_{1}^{\mathrm{A}} \mathrm{s}_{0} \pm\left[\phi, \mathrm{s}_{1}\right] .
$$

With Fubini's theorem we obtain

$$
\begin{aligned}
\mathrm{g}\left(\mathrm{I}^{(s)} \mathrm{K}^{\xi}, \mathrm{X}\right) & =\int_{\mathrm{I}}\left\langle \pm \nabla_{1}^{\mathrm{A}} \xi, \mathrm{s}_{0}\right\rangle+\left\langle \pm[\xi, \phi], \mathrm{s}_{1}\right\rangle+\left\langle\nabla_{3}^{\mathrm{A} \xi}, \mathrm{s}_{2}\right\rangle+\left\langle-\nabla_{2}^{\mathrm{A} \xi, s_{3}}\right\rangle \mathrm{dt} \\
& =\int_{\mathrm{I}}\left\langle\xi, \pm\left[\phi, \mathrm{s}_{1}\right] \mp \nabla_{1}^{A} \mathrm{~s}_{0}+\nabla_{2}^{\mathrm{A}} \mathrm{s}_{3}-\nabla_{3}^{\mathrm{A}} \mathrm{s}_{2}\right\rangle \mathrm{dt} \\
& =\int_{\mathrm{I}}\left\langle\xi, \mathrm{d} \mu_{\mathrm{I}}^{(\mathrm{s})}(\mathrm{X})\right\rangle \mathrm{dt}=\left\langle\xi, \mathrm{d} \mu_{\mathrm{I}}^{(s)}(\mathrm{X})\right\rangle .
\end{aligned}
$$

\subsection{Haydys-Witten Instantons}

Let $M$ be a 5-manifold with (not necessarily integrable) SO(4) structure. Then the alternating product bundles of $M$ split as

$$
\begin{aligned}
& \Lambda^{1} T^{\vee} M \cong \Lambda_{4}^{1} \oplus \Lambda_{1}^{1} \cong \Lambda^{4} T^{\vee} M, \\
& \Lambda^{2} T^{\vee} M \cong \Lambda_{+}^{2} \oplus \Lambda_{-}^{2} \oplus \Lambda_{4}^{2} \cong \Lambda^{3} T^{\vee} M
\end{aligned}
$$

$\Lambda_{1}^{1}$ 's representation is trivial, hence it is the trivial bundle, so we may pick a trivializing section $\eta \in \Gamma\left(\Lambda_{1}^{1}\right)$, and denote by $Y$ the dual of this section. Then $F(\eta)$ is a map identifying $\Lambda_{4}^{1} \cong \Lambda_{4}^{2}$. A quick inspection shows that $G(\eta): \Lambda^{2} \rightarrow \Lambda^{2}$ has the eigenspaces $\Lambda_{+}^{2}, \Lambda_{-}^{2}$ and $\Lambda_{4}^{2}$ corresponding to the eigenvalues $1,-1$ and 0 respectively. 
Definition 6.62. Let $P \rightarrow M$ be a principal G-bundle and let

$$
(A, B) \in \mathcal{C}(P) \times \Omega_{+}^{2}(P, \operatorname{Ad}(P)) .
$$

Then the Haydys-Witten equation are given by

$$
\begin{aligned}
\iota_{Y} F^{A} & =\delta_{A}^{+} B \\
F_{+}^{A} & =\nabla_{Y}^{A} B+\sigma(B, B),
\end{aligned}
$$

where $\delta_{A}^{+}=\operatorname{pr}_{S 11} \circ \nabla^{A}$ and $\sigma$ is induced by $[-,-]_{\mathfrak{s u}(2)} \otimes[-,-]_{\mathfrak{g}}:\left(\Lambda_{+}^{2} \otimes \mathfrak{g}\right) \otimes^{2} \rightarrow \Lambda_{+}^{2} \otimes \mathfrak{g}$.

Remark 6.63. $\nabla^{\mathrm{A}} \mathrm{B}$ lives in $\mathrm{T}^{\vee} \mathrm{M} \otimes \Lambda_{+}^{2} \otimes \mathfrak{g}$, and since in terms of $\mathbf{S O}(4)$-representations $\left(S^{11} \oplus S^{00}\right) \otimes S^{20} \cong S^{31} \oplus S^{11} \oplus S^{20}$, we have a projection onto $S^{11}$. See [Hay15b] for an explicit description of the map.

Similarly we can describe $\sigma$ as the projection onto the $S^{20}$ component of $S^{20} \otimes S^{20} \cong$ $\mathrm{S}^{40} \oplus \mathrm{S}^{20} \oplus \mathrm{S}^{00}$.

Remark 6.64. If $M$ has a hypo structure (again not necessarily integrable), then B decomposes into three sections of the adjoint bundle and the similarities to the Nahm equations are striking.

Remark 6.65. As mentioned, $\iota_{Y}$ identifies $\Lambda_{4}^{2}$ and $\Lambda_{4}^{1}$. Hence the first equation specifies $\mathrm{F}_{4}^{\mathrm{A}}$, whereas the second equations specifies $\mathrm{F}_{+}^{\mathrm{A}}$. This is similar to other monopole equations. The corresponding instantons would be given by $\mathrm{F}_{4}^{\mathrm{A}}=0=\mathrm{F}_{+}^{\mathrm{A}}$.

Remark 6.66. Mazzeo and Witten study possible boundary conditions of the HaydysWitten equations [MW13]. Of particular interest are the same boundary conditions we will introduce in Theorem 8.38, only not on an interval, but on a half line. Furthermore, they consider knots embedded into the boundary and require more complicated conditions on the knot to make a connection to Khovanov homology.

\section{$6.4 \quad G_{2}$-Monopoles}

Let $M$ be a 7-manifold with (not necessarily integrable) $G_{2}$ structure, $P \rightarrow M$ a principal G-bundle and denote the coassociative form of $M$ by $\psi$. Then the alternating product bundle of $M$ decompose as described in Proposition 2.56 .

Definition $6.67\left(\mathbf{G}_{\mathbf{2}}\right.$ Monopoles). A pair $(A, \phi) \in \mathcal{C}(P) \times \Gamma(\operatorname{Ad}(P))$ is called a $\mathbf{G}_{\mathbf{2}^{-}}$ monopole if

$$
\mathrm{F}^{\mathrm{A}} \wedge \psi=\star \mathrm{d}_{\mathrm{A}} \phi
$$

Remark 6.68. Recall that $\Lambda_{7}^{2}$ is the image of $G(\psi)$ restricted to $\Lambda^{1}$. Hence up to this identification the equations are given by $F_{7}^{A}=d_{A} \phi$. The associated instanton equations are given by $F_{7}^{A}=0$. 
Remark 6.69. Oliveira discusses some possible boundary conditions for $\mathbf{G}_{\mathbf{2}}$-Monopoles for examples that fit to our setting in [Oli14]. He considers the boundary condition where $\lim _{r \rightarrow \infty}\|\phi\|<\infty$ exists for a $\mathbf{G}_{2}$-monopole on $\Lambda_{+}^{2} X$ of a 4-manifold, where $r$ is the distance to the zero-section. In addition the existence of a limiting bundle with limiting connection is required. The boundary conditions we are interested in imply these but require more. In the case of Bogomolny equations, these conditions are actually equivalent (see the discussion in Remark 6.43), but this uses the satisfaction of the Bogomolny equations. It is unclear to the author if this can be extended to our case. 


\section{Chapter 7}

\section{An Extended Nahm Transform}

In this chapter we will generalize the Nahm Transform. The Nahm Transform relates a Bogomolny datum (see Definition 6.37) to a Nahm datum (see Definition 6.1). Recalling that both spaces are (formal) hyperkähler reductions of affine spaces, we can picture this as follows,

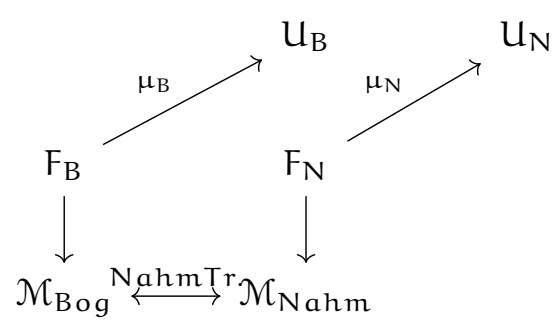

where $F_{B}$ and $F_{N}$ are (affine) flat (infinite dimensional) quaternionic vector spaces and the $\mu_{i}$ are the moment maps. We have in addition used here that the Nahm Transform respects the gauge equivalence class and hyperkähler structure (see e.g. [Nakg1]). The first remark is that the very definition of the Nahm Transform is a transform between the spaces $F_{B}$ and $F_{N}$, which later is shown to respect the gauge classes, and so is well defined on the quotients. Hence we get a transform

$$
\mathrm{F}_{\mathrm{B}} \supset \mu_{\mathrm{B}}^{-1}(0) \leftrightarrow \mu_{\mathrm{N}}^{-1}(0) \subset \mathrm{F}_{\mathrm{N}}
$$

between solutions of the Bogomolny equations and solutions of the Nahm equations (the boundary conditions are encoded in the spaces $F_{i}$ ). We will show that the Nahm Transform is extendable to a tubular neighborhood of $\mu_{i}{ }^{-1}(0) \subset F_{i}$. This means that we relate Bogomolny Data and Nahm Data that satisfy the usual boundary conditions, but satisfy the equations with a small error.

For this we only have to show that the construction remains well defined for small deviations of solutions. In particular we need to show that the boundary conditions remain satisfied. On the other hand we want to show that a small deviation of a solution induces a small deviation on the other side. 
In the first section we will define the necessary notions and in the second section we show that the construction of the Nahm Transform remains valid for small deviations. In the third section we give an approximation of the error term after the transformation and in the last section we will show that the boundary conditions remain satisfied under the small deviations. To show the approximation, we have to give a new elementary proof of the Nahm Transform (of the equation part) without using a choice of a complex structure as e.g. in [Don84] and [Nak91]. This makes the proof shorter and gives a straight forward calculation to verify the equations. Furthermore it highlights that the transform in both directions are virtually identical.

Another possibility to work with this problem would be to define a tubular neighborhood of $\mu^{-1}(0) \subset F$. This would allow to have a well defined projection onto $\mu^{-1}(0)$. In a very similar setting Gaio defined such a projection and moreover a way of mapping $\mu^{-1}(0)$ back into the tubular neighborhood, given a direction and a $\varepsilon$ [Gaig9]. Then adiabatic scaling is used to ensure that the solution of the equations is in this tubular neighborhood. A similar construction would solve the given problem but it seems unclear on how to generalize the results of [Gaig9] to this infinite dimensional setting.

There has been work on defining a tubular neighborhood for the Nahm equations, using a gradient flow in an equivariant setting with cutting and projecting. It is unclear if such a definition will have the required properties to construct an inverse map.

We are interested in right hand errors here, i.e. we don't require a datum to be close to a solution to the equations, but rather that the equations are approximately satisfied. Of course the former implies the latter.

The proof of the boundary conditions in this section follows closely the steps of [Nak91] and [Hit83].

\subsection{Notions}

Let $\mathrm{G}$ be a Lie Group and fix an Ad-invariant scalar product on its Lie Algebra $\mathfrak{g}$.

Definition 7.1. A generalized Nahm datum is an element in $\left(T_{0}, T_{1}, T_{2}, T_{3}\right) \in F_{N}$,

$$
\mu_{N}\left(T_{0}, T_{1}, T_{2}, T_{3}\right)=\eta \in U_{N} .
$$

Definition 7.2. A generalized Bogomolny datum is an element in $(A, \phi) \in F_{B}$,

$$
\mu_{B}(A, \phi)=\eta \in U_{B} .
$$

\subsection{An Extension of the Nahm Transform}

The construction of the extended Nahm Transform is formally equivalent to the usual one. We define the same operator and use the same construction to produce the 
transformed datum. For this to work we only have to make sure that the index of the operator does not change and the kernel remains trivial on the larger class of connections.

Since we will be using the Weitzenböck formulas later on, we also prove those in this section.

\subsubsection{Nahm to Bogomolny}

Definition 7.3 (Nahm Dirac Operator). Let $\left(T_{0}, T_{1}, T_{2}, T_{3}\right)$ be a generalized Nahm datum and $V \rightarrow I$ the associated bundle. For every $x \in \mathbb{R}^{3}$, define

$$
\begin{aligned}
& \mathscr{D}_{x}: \mathbb{H} \otimes W_{0}^{1,2}(\mathrm{I}, \mathrm{V}) \rightarrow \mathbb{H} \otimes \mathrm{L}^{2}(\mathrm{I}, \mathrm{V}) \\
& \mathscr{D}_{x}=\mathbb{1}_{\mathbb{H}} \otimes \nabla_{\mathrm{t}}+\sum_{j=1}^{3}\left(\mathrm{cl}_{j} \otimes\left(\mathrm{T}_{j}-i x_{j} \mathbb{1}_{\mathrm{V}}\right)\right),
\end{aligned}
$$

where $\nabla_{t}=\partial_{t}+T_{0}$ is the connection induced by $T_{0}$ and $\mathrm{cl}_{j}$ are the Clifford multiplication on the spin bundle of $\mathbb{R}^{3}, \mathbf{S}=\underline{\mathbb{H}}$. The tensor product of $\mathbb{H} \cong \mathbb{C}^{2}$ and $V$ is over C.

Lemma 7.4. The adjoint operator of $\mathscr{D}_{\mathrm{x}}$ is given by

$$
\mathscr{D}_{x}^{*}=-\mathbb{1}_{\mathbb{H}} \otimes \nabla_{t}+\sum_{j=1}^{3}\left(c_{j} \otimes\left(T_{j}-i x_{j} \mathbb{1}_{V}\right)\right) .
$$

Proof. We see that

$$
\left(\mathrm{cl}_{\mathrm{j}} \otimes \mathrm{T}_{\mathrm{k}}\right)^{*}=-\mathrm{cl}_{\mathrm{j}} \otimes-\mathrm{T}_{\mathrm{k}}=\mathrm{cl}_{\mathrm{j}} \otimes \mathrm{T}_{\mathrm{k}}
$$

and

$$
\left(\mathrm{cl}_{\mathrm{j}} \otimes i x_{\mathrm{k}}\right)^{*}=-\mathrm{cl}_{\mathrm{j}} \otimes-i \mathrm{x}_{\mathrm{k}}=\mathrm{cl}_{\mathrm{j}} \otimes i x_{\mathrm{k}},
$$

so that the second summand of $\mathscr{D}_{\mathrm{x}}$ is self dual. $\nabla_{\mathrm{t}}$ is a $\mathbf{U}(\mathrm{k})$ connection and as such compatible with a metric from $V$. Using this with the vanishing of the sections on the boundary gives

$$
\left(\mathbb{1}_{\mathbb{H}} \otimes \nabla_{\mathrm{t}}\right)^{*}=-\mathbb{1}_{\mathbb{H}} \otimes \nabla_{\mathrm{t}}
$$

via integration by parts.

Proposition 7.5 (Weitzenböck Formula). Let $\left(T_{0}, T_{1}, T_{2}, T_{3}\right)$ be a generalized Nahm datum, with

$$
\begin{aligned}
& \dot{\mathrm{T}}_{1}+\left[\mathrm{T}_{0}, \mathrm{~T}_{1}\right]+\left[\mathrm{T}_{2}, \mathrm{~T}_{3}\right]=\eta_{1}, \\
& \dot{\mathrm{T}}_{2}+\left[\mathrm{T}_{0}, \mathrm{~T}_{2}\right]+\left[\mathrm{T}_{3}, \mathrm{~T}_{1}\right]=\eta_{2}, \\
& \dot{\mathrm{T}}_{3}+\left[\mathrm{T}_{0}, \mathrm{~T}_{3}\right]+\left[\mathrm{T}_{1}, \mathrm{~T}_{2}\right]=\eta_{3} .
\end{aligned}
$$


Then

$$
\mathscr{D}_{x}^{*} \mathscr{D}_{x}=\mathbb{1}_{\mathbb{H}} \otimes\left(\nabla_{t}^{*} \nabla_{t}+\sum_{j=1}^{3}\left(\mathrm{~T}_{j}-i x_{j}\right)^{*}\left(\mathrm{~T}_{j}-i x_{j}\right)\right)-\mathrm{cl}_{\eta},
$$

where

$$
\mathrm{cl}_{\eta}=\sum_{j=1}^{3} \eta_{i} \mathrm{cl}_{i}
$$

Proof. Define the abbreviating notation

$$
\mathscr{D}_{x}=\mathrm{d}_{\mathrm{t}}+\mathrm{T}+\mathrm{X}
$$

where $d_{t}=\mathbb{1}_{\mathbb{H}} \otimes \nabla_{t}$,

$$
\mathrm{T}=\sum_{j=1}^{3} \mathrm{cl}_{j} \otimes \mathrm{T}_{j} \quad \text { and } \quad \mathrm{X}=-\mathrm{i} \sum_{j=1}^{3} \mathrm{cl}_{j} \otimes \mathrm{x}_{j} \mathbb{1}_{\mathrm{V}} .
$$

Then by Lemma $7 \cdot 4$ we know that $\mathscr{D}_{x}^{*}=-d_{t}+T+X$. We see that

$$
\begin{aligned}
\mathscr{D}_{x}^{*} \mathscr{D}_{x} & =-\mathrm{d}_{\mathrm{t}}^{2}-\mathrm{d}_{\mathrm{t}} \mathrm{T}-\mathrm{T} \mathrm{d}_{\mathrm{t}}-\mathrm{X} \mathrm{d}_{\mathrm{t}}+\mathrm{T} \mathrm{d}_{\mathrm{t}}+\mathrm{T}^{2}+\mathrm{TX}+\mathrm{X} \mathrm{d}_{\mathrm{t}}+\mathrm{XT}+\mathrm{X}^{2} \\
& =-\mathrm{d}_{\mathrm{t}}^{2}-\mathrm{d}_{\mathrm{t}} \mathrm{T}+\mathrm{T}^{2}+\mathrm{TX}+\mathrm{X} \mathrm{T}+\mathrm{X}^{2}
\end{aligned}
$$

We now inspect the terms. Starting with

$$
\mathrm{d}_{\mathrm{t}} \mathrm{T}=\sum_{\mathrm{j}=1}^{3} \mathrm{cl}_{j} \otimes \nabla_{\mathrm{t}} \mathrm{T}_{\mathrm{j}}
$$

and continue with

$$
X^{2}=-\sum_{i, j=1}^{3} \mathrm{cl}_{i} \mathrm{cl}_{j} \otimes \mathrm{x}_{i} x_{j}=\mathbb{1}_{\mathrm{C}^{2}} \otimes \sum_{i=1}^{3} \mathrm{x}_{i}^{2}-\underbrace{\sum_{i \neq j} \mathrm{cl}_{i} \mathrm{cl}_{j} \otimes \mathrm{x}_{i} x_{j}}_{=0},
$$

where the second term vanishes because the $\mathrm{cl}_{i}$ anticommute. For XT $+\mathrm{TX}$ realize that $\mathrm{cl}_{j} \otimes \mathrm{T}_{j}$ and $\mathrm{cl}_{i} \otimes \mathrm{x}_{i}$ anticommute if $i \neq j$, so that the corresponding terms of TX and XT cancel. Only the diagonal terms survive, i.e.

$$
X \mathrm{~T}+\mathrm{TX}=2 i \mathbb{1}_{\mathrm{H}} \otimes \sum_{j=1}^{3} \mathrm{~T}_{j} x_{j} .
$$

Finally we need to look at

$$
\mathrm{T}^{2}=\sum_{i, j=1}^{3} \mathrm{cl}_{i} \mathrm{cl}_{j} \otimes \mathrm{T}_{i} \mathrm{~T}_{j}=-\mathbb{1}_{\mathbb{H}} \otimes \sum_{j=1}^{3} \mathrm{~T}_{j}^{2}+\sum_{i \neq j} \mathrm{cl}_{i} \mathrm{cl}_{j} \otimes \mathrm{T}_{i} \mathrm{~T}_{j} .
$$


For the second term we continue

$$
\begin{aligned}
\sum_{i \neq j} \mathrm{cl}_{i} \mathrm{cl}_{j} \otimes \mathrm{T}_{i} \mathrm{~T}_{j} & =\sum_{1 \leqslant i<j \leqslant 3} \mathrm{cl}_{i} \mathrm{cl}_{j} \otimes\left(\mathrm{T}_{i} \mathrm{~T}_{j}-\mathrm{T}_{j} \mathrm{~T}_{i}\right) \\
& =-\mathrm{cl}_{1} \otimes\left[\mathrm{T}_{2}, \mathrm{~T}_{3}\right]-\mathrm{cl}_{2} \otimes\left[\mathrm{T}_{3}, \mathrm{~T}_{1}\right]-\mathrm{cl}_{3} \otimes\left[\mathrm{T}_{1}, \mathrm{~T}_{2}\right] .
\end{aligned}
$$

We conclude that

$$
\begin{aligned}
(T+X)^{2} & =\mathbb{1}_{C^{2}} \otimes\left(\sum_{j=1}^{3}\left(x_{j}^{2}+2 i T_{j} x_{j}-T_{j}^{2}\right)\right)+L \\
& =\mathbb{1}_{C^{2}} \otimes\left(\sum_{j=1}^{3}\left(T_{j}-i x_{j}\right)^{*}\left(T_{j}-i x_{j}\right)\right)+L,
\end{aligned}
$$

where $L$ is defined by (7.24),

$$
-\mathrm{L}=\mathrm{cl}_{1} \otimes\left[\mathrm{T}_{2}, \mathrm{~T}_{3}\right]+\mathrm{cl}_{2} \otimes\left[\mathrm{T}_{3}, \mathrm{~T}_{1}\right]+\mathrm{cl}_{3} \otimes\left[\mathrm{T}_{1}, \mathrm{~T}_{2}\right] .
$$

This shows that

$$
\mathscr{D}_{x}^{*} \mathscr{D}_{x}=\mathbb{1}_{\mathbb{H}} \otimes\left(\nabla_{\mathrm{t}}^{*} \nabla_{\mathrm{t}}+\sum_{j=1}^{3}\left(\mathrm{~T}_{j}-\mathfrak{i} \mathrm{x}_{\mathrm{j}}\right)^{*}\left(\mathrm{~T}_{\mathrm{j}}-\mathfrak{i} \mathrm{x}_{\mathrm{j}}\right)\right)-\mathrm{d}_{\mathrm{t}} \mathrm{T}+\mathrm{L} .
$$

Plugging in the Nahm equations 7.10 through 7.12 shows that

$$
L-d_{t} T=-\sum_{j=1}^{3} \mathrm{cl}_{j} \otimes \eta_{j}
$$

which completes the proof.

We recover the Weitzenböck formula in [Nak91] by letting $\eta_{j}=0$.

Corollary 7.6. The $\mathrm{L}^{2}$ kernel of the family $\mathscr{D}_{x}$ vanishes for an approximate solution of the Bogomolny equations.

$$
\text { ker } \mathscr{D}_{x}=\{0\} \text {. }
$$

Proof. Using the Weitzenböck formula for $\sigma=s \otimes e \in \Gamma(S \otimes E)$, we see that right hand side is a positive operator if we assume $\eta$ to be small enough. So $\mathscr{D}_{x} \sigma=0$ if and only if $\|s \otimes e\|=0$.

Corollary 7.7. We have,

$$
\operatorname{dim} \operatorname{ker} \mathscr{D}_{x}^{*}=\operatorname{dim} \operatorname{coker} \mathscr{D}_{x}=\text { ind } \mathscr{D}_{x},
$$

where ind is the index of the differential operator. 
Theorem 7.8 ([Hit83]). For any generalized Nahm datum, we have

$$
\text { ind } \mathscr{D}_{x}=2 \text {. }
$$

Remark 7.9. The $\mathrm{L}^{2}$-kernel of $\mathscr{D}_{x}$ gives a 2-dimensional subspace in $\mathrm{E}_{\mathrm{x}} \subset \mathbb{H} \otimes \mathrm{L}^{2}(\mathrm{I}, \mathrm{V})$ for every $x \in \mathbb{R}^{3}$. This may be interpreted as a 2-dimensional subbundle of the infinite dimensional trivial bundle

$$
\mathrm{E} \subset \underline{\mathrm{L}^{2}(\mathrm{I}, \mathrm{S} \otimes \mathrm{V})} \rightarrow \mathbb{R}^{3} .
$$

Definition 7.10. Denote by $\pi_{x}: L^{2}(I, S \otimes V) \rightarrow E_{x}$ the orthogonal projection with respect to the $\mathrm{L}^{2}$ scalar product and by $\mathrm{l}: \mathrm{E} \rightarrow \underline{\mathrm{L}^{2}(\mathrm{I}, \mathrm{S} \otimes \mathrm{V})}$ the inclusion. Define the connection on $\mathrm{E}$ by

$$
\mathrm{d}_{\mathrm{A}}: \Gamma\left(\mathbb{R}^{3}, \mathrm{E}\right) \stackrel{\mathrm{\iota}}{\rightarrow} \Gamma\left(\mathrm{I}, \underline{\mathrm{L}^{2}(\mathrm{I}, \mathbf{S} \otimes \mathrm{V})}\right) \stackrel{\mathrm{d}}{\rightarrow} \Gamma\left(\mathrm{I}, \underline{\mathrm{L}^{2}(\mathrm{I}, \mathbf{S} \otimes \mathrm{V})}\right) \stackrel{\stackrel{\pi}{\rightarrow}}{ } \Gamma\left(\mathbb{R}^{3}, \mathrm{E}\right) .
$$

Further define the endomorphism of $E$ by

$$
\varphi: \Gamma\left(\mathbb{R}^{3}, E\right) \rightarrow \Gamma\left(\mathbb{R}^{3}, E\right), \quad s \mapsto \pi(\text { its }) \quad k \in\{1,2,3\} .
$$

\subsubsection{Bogomolny to Nahm}

Definition 7.11 (Bogomolny Dirac Operator). Let $(A, \phi)$ be a generalized Bogomolny datum and $E \rightarrow \mathbb{R}^{3}$ the associated bundle. For every $t \in I=(-1,1)$, define

$$
\mathscr{D}_{\mathrm{A}, \mathrm{t}}=\mathscr{D}_{\mathrm{A}}+(\phi-i t): \Gamma(\mathbf{S} \otimes \mathrm{E}) \rightarrow \Gamma(\mathbf{S} \otimes \mathrm{E})
$$

where $S \rightarrow \mathbb{R}^{3}$ is the spin bundle of $\mathbb{R}^{3}$.

Lemma 7.12. The formal adjoint of $\mathscr{D}_{\mathrm{A}, \mathrm{t}}$ is given by

$$
\mathscr{D}_{\mathrm{A}, \mathrm{t}}^{\star}=\mathscr{D}_{\mathrm{A}}-(\phi-i \mathrm{t}) .
$$

Proof. Note that $\mathscr{D}_{\mathrm{A}}$ is self-adjoint, and $\phi-i \mathrm{it} \in \mathfrak{u}(\mathbf{S} \otimes \mathrm{E})$.

Proposition 7.13 (Weitzenböck Formula). Let $(A, \phi)$ be a generalized Bogomolny datum, with

$$
\star \mathrm{F}^{\mathrm{A}}-\mathrm{d}_{\mathrm{A}} \varphi=\eta, \quad \eta=\left(\eta_{1}, \eta_{2}, \eta_{3}\right) \in \Omega^{1}\left(\mathbb{R}^{3}, \mathfrak{g}\right) .
$$

The Bogomolny Dirac operator satisfies the following Weitzenböck formula

$$
\mathscr{D}_{\mathrm{A}, \mathrm{t}}^{*} \mathscr{D}_{\mathrm{A}, \mathrm{t}}=\nabla^{*} \nabla-\mathbb{1}_{\mathrm{S}} \otimes(\phi-i t \mathbb{1})^{2}-\mathrm{cl}_{\eta},
$$

where

$$
\mathrm{cl}_{\eta}=\sum_{i=1}^{3} \eta_{i} \mathrm{cl}_{i}
$$


Proof. Let $\psi=\phi-i t 1$ for this proof. By definition

$$
\mathscr{D}_{\mathrm{A}, \mathrm{t}}^{*} \mathscr{D}_{\mathrm{A}, \mathrm{t}}=\mathscr{D}_{\mathrm{A}}^{*} \mathscr{D}_{\mathrm{A}}+\mathscr{D}_{\mathrm{A}}^{*}\left(\mathbb{1}_{\mathrm{S}} \otimes \psi\right)-\left(\mathbb{1}_{\mathrm{S}} \otimes \psi\right) \mathscr{D}_{\mathrm{A}}-\mathbb{1}_{\mathrm{S}} \otimes \psi^{2} .
$$

Using the Weitzenböck formula of $\mathscr{D}_{A}$, we see that

$$
\mathscr{D}_{\mathrm{A}}^{*} \mathscr{D}_{\mathrm{A}}=\nabla^{*} \nabla+\frac{1}{4} \mathrm{Sc}+\frac{1}{2} \mathscr{R},
$$

where

$$
\mathscr{R}(\mathrm{s} \otimes e)=\sum_{i, j} \mathrm{cl}_{i} \mathrm{cl}_{j} \mathrm{~s} \otimes \mathrm{F}^{\mathrm{A}}\left(\mathrm{e}^{\mathrm{i}}, e^{\mathrm{j}}\right) e,
$$

$\left(e^{i}\right)$ are standard coordinates and via $\mathrm{Ad}(\mathrm{P}) \rightarrow \operatorname{End}(\mathrm{E})$ we identify $\mathrm{F}^{\mathrm{A}}\left(e^{i}, e^{j}\right)=\mathrm{F}_{i j}^{\mathrm{A}}$ with an endomorphism of $\mathrm{E} . \mathrm{Cl}_{i}$ is a short notation for $\mathrm{cl}_{\mathrm{e}^{i}}$ and $\nabla$ is the connection on $\mathbf{S} \otimes \mathrm{E}$ induced by the Levi Civita connection and $\nabla_{\mathrm{A}}$. Since $\mathbb{R}^{3}$ is flat, $\mathrm{Sc}=0$ and we get

$$
\begin{aligned}
\mathscr{D}_{\mathrm{A}}(\mathrm{s} \otimes \psi e) & =\mathrm{cl} \nabla(\mathrm{s} \otimes \psi e) \\
& =\mathrm{cl}\left(\nabla_{\mathrm{LC}} \mathrm{s} \otimes \psi e+\mathrm{s} \otimes \nabla_{\mathrm{A}}[\psi e]\right) \\
& =\mathscr{D} \mathrm{s} \otimes \psi e+\mathrm{cl}\left(\mathrm{s} \otimes\left(\left[\mathrm{d}_{\mathrm{A}} \psi\right] e+\psi \nabla_{\mathrm{A}} e\right)\right) \\
& =\mathbb{1}_{\mathrm{S}} \otimes \psi\left(\mathscr{D}_{\mathrm{A}}(\mathrm{s} \otimes e)\right)+\mathrm{cl}\left(\mathrm{s} \otimes\left[\mathrm{d}_{\mathrm{A}} \psi\right] e\right),
\end{aligned}
$$

so that

$$
\mathscr{D}_{\mathrm{A}}\left(\mathbb{1}_{\mathrm{S}} \otimes \psi\right)-\left(\mathbb{1}_{\mathrm{S}} \otimes \psi\right) \mathscr{D}_{\mathrm{A}}=\operatorname{cl}\left(\mathbb{1}_{\mathrm{S}} \otimes \mathrm{d}_{\mathrm{A}} \psi\right) .
$$

Note that $d_{A} \psi=d_{A} \phi=\star F^{A}-\eta$, hence

$$
\begin{aligned}
\mathrm{cl}\left(\mathbb{1}_{S} \otimes \mathrm{d}_{A} \psi\right)(\mathrm{s} \otimes e)= & \mathrm{cl}\left(\mathbb { 1 } _ { \mathrm { S } } \otimes \left(\left(\mathrm{F}_{12}^{\mathrm{A}}-\eta_{3}\right) e^{3}+\left(\mathrm{F}_{23}^{\mathrm{A}}-\eta_{1}\right) e^{1}\right.\right. \\
& \left.\left.+\left(\mathrm{F}_{31}^{\mathrm{A}}-\eta_{2}\right) e^{2}\right)\right)(\mathrm{s} \otimes e) \\
= & \mathrm{cl}_{3} \mathrm{~s} \otimes \mathrm{F}_{12}^{\mathrm{A}} \mathrm{e}+\mathrm{cl}_{1} \mathrm{~s} \otimes \mathrm{F}_{23}^{\mathrm{A}} \mathrm{e}+\mathrm{cl}_{2} \mathrm{~s} \otimes \mathrm{F}_{31}^{\mathrm{A}} e \\
& \quad-\sum_{i=1}^{3} \eta_{i} \mathrm{cl}_{i}(\mathrm{~s} \otimes e) .
\end{aligned}
$$

On the other hand, we can calculate that

$$
\frac{1}{2} \mathscr{R}(\mathrm{s} \otimes e)=\sum_{i<j} \mathrm{cl}_{i} \mathrm{cl}_{j} \mathrm{~s} \otimes \mathrm{F}_{i j}^{\mathrm{A}} \mathrm{e}=-\mathrm{cl}_{3} \mathrm{~s} \otimes \mathrm{F}_{12}^{\mathrm{A}} \mathrm{e}+\mathrm{cl}_{2} \mathrm{~s} \otimes \mathrm{F}_{13}^{\mathrm{A}} \mathrm{e}-\mathrm{cl}_{1} \mathrm{~s} \otimes \mathrm{F}_{23}^{\mathrm{A}} \mathrm{e},
$$

where we have used that $\mathrm{cl}_{1}, \mathrm{cl}_{2}, \mathrm{cl}_{3}$ is multiplication by $-i,-j,-k \in \mathbb{H}$. Therefore these terms cancel and we end up with

$$
\mathscr{D}_{\mathrm{A}, \mathrm{t}}^{*} \mathscr{D}_{\mathrm{A}, \mathrm{t}}=\nabla^{*} \nabla-\mathbb{1}_{\mathrm{S}} \otimes(\phi-i \mathrm{t} \mathbb{1})^{2}-\sum_{i=1}^{3} \eta_{\mathrm{i}} \mathrm{cl}_{\mathrm{i}} .
$$


We recover the Weitzenböck formula in [Nak91] by letting $\eta_{j}=0$.

Remark 7.14. Note that the spin bundle of $\mathbb{R}^{3}, \mathbf{S} \rightarrow \mathbb{R}^{3}$, is the trivial bundle $\underline{\mathbb{H}}$. As such, we can identify $\Gamma\left(\mathbb{R}^{3}, S \otimes E\right) \cong \mathbb{H} \otimes \Gamma\left(\mathbb{R}^{3}, E\right)$. In this identification, the Dirac operator now becomes

$$
\mathscr{D}_{\mathrm{A}}=\mathrm{cl} \circ \nabla^{\mathrm{A}},
$$

and the Weitzenböck Formula becomes

$$
\mathscr{D}_{\mathrm{A}, \mathrm{t}}^{*} \mathscr{D}_{\mathrm{A}, \mathrm{t}}=\mathbb{1}_{\mathbb{H}} \otimes\left(\nabla^{\mathrm{A}}\right)^{*} \nabla^{\mathrm{A}}-\mathbb{1}_{\mathbb{H}} \otimes(\phi-i \mathrm{t} \mathbb{1})^{2}-\sum_{i=1}^{3} \eta_{i} \mathrm{cl}_{i} .
$$

This shows that in this identification and with $\eta_{i}=0, \mathscr{D}_{\mathrm{A}, \mathrm{t}}^{*} \mathscr{D}_{\mathrm{A}, \mathrm{t}}$ (hence also its inverse) commutes with $\mathrm{cl}_{i}$. This is the essential fact to show that the transformed datum satisfies the equations.

Corollary 7.15. The $\mathrm{L}^{2}$ kernel of the family $\mathscr{D}_{\mathrm{A}, \mathrm{t}}$ vanishes for an approximate solution of the Bogomolny equations.

$$
\operatorname{ker} \mathscr{D}_{\mathrm{A}, \mathrm{t}}=\{0\}
$$

Proof. Using the Weitzenböck formula for $\sigma=s \otimes e \in \Gamma(S \otimes E)$, we see that right hand side is a positive operator if we assume $\eta$ to be small enough (i.e. $\mathrm{cl}_{\eta}: W^{1,2} \rightarrow \mathrm{L}^{2}$ is small enough in the corresponding operator norm) depending on $A$ and $\phi$. So $\mathscr{D}_{\mathrm{A}, \mathrm{t}} \sigma=0$ if and only if $\|s \otimes e\|_{\mathrm{L}^{2}}=0$.

Corollary 7.16. We have,

$$
\operatorname{dim} \operatorname{ker} \mathscr{D}_{\mathrm{A}, \mathrm{t}}^{*}=\operatorname{dim} \operatorname{coker} \mathscr{D}_{\mathrm{A}, \mathrm{t}}=\operatorname{ind} \mathscr{D}_{\mathrm{A}, \mathrm{t}},
$$

where ind is the index of the differential operator.

Theorem 7.17 (Callias [Cal78]). For any generalized Bogomolny datum we have

$$
\text { ind } \mathscr{D}_{\mathrm{A}, \mathrm{t}}=\mathrm{k} \text {. }
$$

Remark 7.18. The $\mathrm{L}^{2}$-kernel of $\mathscr{D}_{\mathrm{A}, \mathrm{t}}$ gives a k-dimensional subspace in $\mathrm{V}_{\mathrm{t}} \subset \mathrm{L}^{2}\left(\mathbb{R}^{3}, \mathbf{S} \otimes\right.$ E) for every $t \in(-1,1)=I$. This may be interpreted as a $k$-dimensional subbundle of the infinite dimensional trivial bundle

$$
\mathrm{V} \subset \underline{\mathrm{L}^{2}\left(\mathbb{R}^{3}, \mathrm{~S} \otimes \mathrm{E}\right)} \rightarrow \mathrm{I} .
$$

Definition 7.19. Denote by $\pi_{t}: L^{2}\left(\mathbb{R}^{3}, S \otimes E\right) \rightarrow V_{t}$ the orthogonal projection with respect to the $\mathrm{L}^{2}$ scalar product and by $\mathrm{l}: \mathrm{V} \rightarrow \underline{\mathrm{L}^{2}\left(\mathbb{R}^{3}, S \otimes E\right)}$ the inclusion. Define the connection on $\mathrm{V}$ by

$$
\nabla_{\mathrm{t}}: \Gamma(\mathrm{I}, \mathrm{V}) \stackrel{\mathrm{l}}{\rightarrow} \Gamma\left(\mathrm{I}, \underline{\mathrm{L}^{2}\left(\mathbb{R}^{3}, \mathrm{~S} \otimes \mathrm{E}\right)}\right) \stackrel{\mathrm{d}}{\rightarrow} \Gamma\left(\mathrm{I}, \underline{\mathrm{L}^{2}\left(\mathbb{R}^{3}, \mathbf{S} \otimes \mathrm{E}\right)}\right) \stackrel{\pi}{\rightarrow} \Gamma(\mathrm{I}, \mathrm{V}) .
$$

Further define three endomorphisms of $\mathrm{V}$ by

$$
\mathrm{T}_{\mathrm{k}}: \Gamma(\mathrm{I}, \mathrm{V}) \rightarrow \Gamma(\mathrm{I}, \mathrm{V}), \quad \mathrm{s} \mapsto \pi\left(\mathrm{ix}_{\mathrm{k}} \mathrm{s}\right) .
$$




\subsection{Error Approximation}

Let us begin by some general remarks. First off, note that the orthogonal projection onto the kernel of $\mathscr{D}_{A, t / x}^{*}$ along the image of $\mathscr{D}_{A, t / x}$ (where we drop the subscript) is given by

$$
\pi=\mathbb{1}-\mathscr{D}\left(\mathscr{D}^{*} \mathscr{D}\right)^{-1} \mathscr{D}^{*}
$$

This implies immediately that the image of $\mathscr{D}$ is in the kernel of $\pi$, indeed,

$$
\pi(\mathscr{D} \psi)=\mathscr{D} \psi-\mathscr{D}\left(\mathscr{D}^{*} \mathscr{D}\right)^{-1} \mathscr{D}^{*} \mathscr{D} \psi=\mathscr{D} \psi-\mathscr{D} \psi=0 .
$$

Both the Bogomolny equations and the Nahm equations are vector bundle endomorphism valued. To show that they are satisfied, we pick an arbitrary element $\psi \in \operatorname{ker} \mathscr{D}^{*}$, apply the equation and show that the result is zero. Plugging in the definitions of the transformed data, we see that the last step of the equation is applying the projection $\pi$, so we use two tricks to drop terms. First, anything in the image of $\mathscr{D}$ vanishes, because of the calculation above, and second whenever $\mathscr{D}^{*}$ gets applied to $\psi$, the term vanishes.

It then turns out that we merely have to calculate some commutators, to apply the facts above. The final step uses the Weitzenböck formula that we have shown for the generalized Laplacian $\mathscr{D}^{*} \mathscr{D}$. The important part about these is that they commute with the Clifford multiplications $\mathrm{cl}_{i}$.

\section{7·3.1 Nahm to Bogomolny}

Theorem 7.20. If $\left(T_{0}, T_{1}, T_{2}, T_{3}\right)$ satisfy the Nahm equations, then the transformed datum $\left(\mathrm{d}_{\mathrm{A}}, \phi\right)$ satisfies the Bogomolny Equations.

Proof. Recall the Bogomolny equations

$$
\mathrm{F}^{\mathrm{A}}=\star \mathrm{d}_{\mathrm{A}} \phi .
$$

In coordinates we may write this as

$$
\mathrm{F}_{23}^{\mathrm{A}}=\nabla_{1}^{\mathrm{A}} \phi, \quad \mathrm{F}_{31}^{\mathrm{A}}=\nabla_{2}^{\mathrm{A}} \phi, \quad \mathrm{F}_{12}^{\mathrm{A}}=\nabla_{3}^{\mathrm{A}} \phi .
$$

Let $\psi \in \operatorname{ker} \mathscr{D}_{x}^{*}$. Then we can apply the equations to $\psi$ to get

$$
\begin{aligned}
& \left(\nabla_{1}^{\mathrm{A}} \phi\right) \psi-\left[\nabla_{2}^{\mathrm{A}}, \nabla_{3}^{\mathrm{A}}\right] \psi=0 \\
& \left(\nabla_{2}^{\mathrm{A}} \phi\right) \psi-\left[\nabla_{3}^{\mathrm{A}}, \nabla_{1}^{\mathrm{A}}\right] \psi=0 \\
& \left(\nabla_{3}^{\mathrm{A}} \phi\right) \psi-\left[\nabla_{1}^{\mathrm{A}}, \nabla_{2}^{\mathrm{A}}\right] \psi=0 .
\end{aligned}
$$

Plugging in the definitions and using

$$
\left(\nabla_{1}^{\mathrm{A}} \phi\right) \psi=\nabla_{1}^{\mathrm{A}}(\phi(\psi))-\phi\left(\nabla_{1}^{\mathrm{A}} \psi\right),
$$


we arrive to the equivalent form of the first equations

$$
\pi\left(\partial_{1} \pi(i t \psi)\right)-\pi\left(i t \pi\left(\partial_{1} \psi\right)\right)-\pi\left(\partial_{2} \pi\left(\partial_{3} \psi\right)\right)+\pi\left(\partial_{3} \pi\left(\partial_{2} \psi\right)\right)=0 .
$$

It suffices to show that

$$
\partial_{1} \pi(i t \psi)-i t \pi\left(\partial_{1} \psi\right)-\partial_{2} \pi\left(\partial_{3} \psi\right)+\partial_{3} \pi\left(\partial_{2} \psi\right) \in \operatorname{ker} \pi
$$

Using the fact that $\pi=1-\mathscr{D}_{x}\left(\mathscr{D}_{x}^{*} \mathscr{D}_{x}\right)^{-1} \mathscr{D}_{x}^{*}$, we end up with

$$
\begin{aligned}
& -\partial_{1} \mathscr{D}_{x}\left(\mathscr{D}_{x}^{*} \mathscr{D}_{x}\right)^{-1} \mathscr{D}_{x}^{*}(i t \psi)+i t \mathscr{D}_{x}\left(\mathscr{D}_{x}^{*} \mathscr{D}_{x}\right)^{-1} \mathscr{D}_{x}^{*}\left(\partial_{1} \psi\right) \\
& +\partial_{2} \mathscr{D}_{x}\left(\mathscr{D}_{x}^{*} \mathscr{D}_{x}\right)^{-1} \mathscr{D}_{x}^{*}\left(\partial_{3} \psi\right)-\partial_{3} \mathscr{D}_{x}\left(\mathscr{D}_{x}^{*} \mathscr{D}_{x}\right)^{-1} \mathscr{D}_{x}^{*}\left(\partial_{2} \psi\right) .
\end{aligned}
$$

Now permuting $\mathscr{D}_{x}$ with the other operators, we calculate

$$
\left[\mathscr{D}_{x}, \partial_{\mathrm{j}}\right]=\mathrm{cl}_{\mathrm{j}} \otimes \mathrm{i}, \quad\left[\mathscr{D}_{x}, \mathrm{it}\right]=\mathrm{i} .
$$

Using these brackets we arrive at

$$
\begin{array}{cc}
\mathrm{cl}_{1} \otimes i\left(\mathscr{D}_{x}^{*} \mathscr{D}_{x}\right)^{-1} \mathscr{D}_{x}^{*}(i t \psi)-\mathfrak{i}\left(\mathscr{D}_{x}^{*} \mathscr{D}_{x}\right)^{-1} \mathscr{D}_{x}^{*}\left(\partial_{1} \psi\right) & \operatorname{modim} \mathscr{D}_{x} . \\
-\mathrm{cl}_{2} \otimes i\left(\mathscr{D}_{x}^{*} \mathscr{D}_{x}\right)^{-1} \mathscr{D}_{x}^{*}\left(\partial_{3} \psi\right)+\mathrm{cl}_{3} \otimes i\left(\mathscr{D}_{x}^{*} \mathscr{D}_{x}\right)^{-1} \mathscr{D}_{x}^{*}\left(\partial_{2} \psi\right) &
\end{array}
$$

Next up we use the commutators with $\mathscr{D}^{*}$

$$
\left[\mathscr{D}_{x}^{*}, \partial_{\mathrm{j}}\right]=\mathrm{cl}_{\mathrm{j}} \otimes \mathrm{i}, \quad\left[\mathscr{D}_{x}^{*}, \mathrm{it}\right]=-\mathrm{i},
$$

to get modulo ker $\mathscr{D}_{x}^{*}$

$$
\begin{aligned}
& \left(\mathrm{cl}_{1} \otimes \mathbb{1}_{V}\right)\left(\mathscr{D}_{x}^{*} \mathscr{D}_{x}\right)^{-1} \psi+\left(\mathscr{D}_{x}^{*} \mathscr{D}_{x}\right)^{-1}\left(\mathrm{cl}_{1} \otimes \mathbb{1}_{\mathrm{V}}\right) \psi \\
& +\left(\mathrm{cl}_{2} \otimes \mathbb{1}_{\mathrm{V}}\right)\left(\mathscr{D}_{x}^{*} \mathscr{D}_{x}\right)^{-1}\left(\mathrm{cl}_{3} \otimes \mathbb{1}_{\mathrm{V}}\right) \psi-\left(\mathrm{cl}_{3} \otimes \mathbb{1}_{\mathrm{V}}\right)\left(\mathscr{D}_{x}^{*} \mathscr{D}_{\mathrm{x}}\right)^{-1}\left(\mathrm{cl}_{2} \otimes \mathbb{1}_{\mathrm{V}}\right) \psi
\end{aligned}
$$

Note that we have not used that $\left(T_{0}, T_{1}, T_{2}, T_{3}\right)$ satisfy the Nahm equations yet. Only now do we need the fact, that

$$
\left(\mathscr{D}^{*} \mathscr{D}\right)^{-1}=\mathbb{1}_{\mathbf{S}} \otimes \mathrm{G},
$$

where $G$ is the Greens Operator of the right rand side of the Weitzenböck formula 7.5 for vanishing error $\eta$. We arrive at

$$
(\underbrace{\left(\mathrm{cl}_{1}+\mathrm{cl}_{1}+\mathrm{cl}_{2} \mathrm{cl}_{3}-\mathrm{cl}_{3} \mathrm{cl}_{2}\right)}_{2 \mathrm{cl}_{1}-\mathrm{cl}_{1}-\mathrm{cl}_{1}=0} \otimes \mathbb{1}_{\mathrm{V}})\left(\mathscr{D}_{x}^{*} \mathscr{D}_{\chi}\right)^{-1} \psi,
$$

which finishes the proof. To prove the other equations, cyclically permute 1,2 and 3 in this proof. 
Corollary 7.21. For a generalized Nahm datum $\left(\mathrm{T}_{0}, \mathrm{~T}_{1}, \mathrm{~T}_{2}, \mathrm{~T}_{3}\right)$ with the error term satisfying $\left\|\mathrm{cl}_{\eta}\right\|<\left\|\mathrm{G}_{0}\right\|^{-1}$ (in the operator norm from $\mathrm{W}^{1,2}$ to $\mathrm{W}^{1,2}$ ), the transformed error term is given by

$$
\psi \mapsto \pi \circ\left(\begin{array}{l}
\mathrm{Ecl}_{1}+\mathrm{cl}_{1} \mathrm{E}+\mathrm{cl}_{2} \mathrm{Ecl}_{3}-\mathrm{cl}_{3} \mathrm{Ecl}_{2} \\
\mathrm{Ecl}_{2}+\mathrm{cl}_{2} \mathrm{E}+\mathrm{cl}_{3} \mathrm{Ecl}_{1}-\mathrm{cl}_{1} \mathrm{Ecl}_{3} \\
\mathrm{Ecl}_{3}+\mathrm{cl}_{3} \mathrm{E}+\mathrm{cl}_{1} \mathrm{Ecl}_{2}-\mathrm{Cl}_{2} \mathrm{Ecl}_{1}
\end{array}\right) \psi
$$

where

$$
E=\left(\sum_{n=1}^{\infty}\left(G_{0} c_{\eta}\right)^{n}\right) G_{0}
$$

$\mathrm{G}_{0}$ is the Greens operator of

$$
\mathbb{1}_{\mathbb{H}} \otimes\left(\nabla_{\mathrm{t}}^{*} \nabla_{\mathrm{t}}+\sum_{j=1}^{3}\left(\mathrm{~T}_{j}-i x_{j}\right)^{*}\left(\mathrm{~T}_{\mathrm{j}}-i x_{j}\right)\right)
$$

and for a section $\psi \in \Gamma\left(\mathbb{R}^{3}, E\right)$.

Proof. Let now $\mu_{N}\left(T_{0}, T_{1}, T_{2}, T_{3}\right)=\eta$. We have shown, that the Weitzenböck formula is given by

$$
\mathscr{D}_{x}^{*} \mathscr{D}_{x}=\mathbb{1}_{\mathbb{H}} \otimes\left(\nabla_{\mathrm{t}}^{*} \nabla_{\mathrm{t}}+\sum_{j=1}^{3}\left(\mathrm{~T}_{j}-i x_{j}\right)^{*}\left(\mathrm{~T}_{\mathrm{j}}-i x_{j}\right)\right)-\mathrm{cl}_{\eta} .
$$

Let us denote this by $\mathrm{G}_{0}^{-1}-\mathrm{cl}_{\eta}$, where $\mathrm{G}_{0}$ (the Greens operator) commutes with the Clifford multiplication. We derive an expression of the Green operator $\mathrm{G}$ of $\mathscr{D}_{x}^{*} \mathscr{D}_{x}$ in terms of the Green operator $G_{0}$. We see then, that

$$
\mathscr{D}_{x}^{*} \mathscr{D}_{x}=\mathrm{G}_{0}^{-1}\left(1-\mathrm{G}_{0} \mathrm{cl}_{\eta}\right),
$$

so that for $\left\|\mathrm{cl}_{\eta}\right\|<\left\|\mathrm{G}_{0}\right\|^{-1}$,

$$
\mathrm{G}=\left(1-\mathrm{G}_{0} \mathrm{cl}_{\eta}\right)^{-1} \mathrm{G}_{0}=\left(\sum_{n=0}^{\infty}\left(\mathrm{G}_{0} \mathrm{cl}_{\eta}\right)^{\mathrm{n}}\right) \mathrm{G}_{0}=\mathrm{G}_{0}+\mathrm{E},
$$

where $\mathrm{G}$ is the Greens operator of $\mathscr{D}_{x}^{*} \mathscr{D}_{x}$ and $\mathrm{E}$ is defined by the equation above. By looking at equations $(7.79)$ and $(7.80)$, we see that the error term's first component is given by

$$
\pi\left(\left(\mathrm{Ecl}_{1}+\mathrm{cl}_{1} \mathrm{E}+\mathrm{cl}_{2} \mathrm{Ecl}_{3}-\mathrm{Cl}_{3} \mathrm{Ecl}_{2}\right) \psi\right)
$$

The others follow similarly.

Corollary 7.22. If $\left\|\mathrm{cl}_{\eta}\right\|<\left\|\mathrm{G}_{0}\right\|^{-1}$ (in the operator norm from $\mathrm{W}^{1,2}$ to $\mathrm{W}^{1,2}$ ), the transformed error term $\eta_{\mathrm{Bog}}$ is in $\mathcal{O}\left(\mathrm{r}^{-4}\right)$. 
Proof. Let us denote the operator (7.85) by $X$. Then $X G_{0}=\mathbb{1}$, and we see that $\|X \psi\| \geqslant$ $\mathrm{Cr}^{2}\|\psi\|$ by the definition of $X$ for $r$ large enough and some constant $C$ (compare to argument for equation (7.126). Using this for $\psi=G_{0} \psi$ shows that

$$
\mathrm{Cr}^{2}\left\|\mathrm{G}_{0} \psi\right\|^{2} \leqslant\left\|X \mathrm{G}_{0} \psi\right\|^{2}=\|\psi\|^{2} .
$$

We conclude that $\left\|G_{0}\right\|=\mathcal{O}\left(r^{-2}\right)$. This implies that $E=\mathcal{O}\left(r^{-4}\right)$, which implies the claim (note that $\eta_{\mathrm{Nahm}}$ is independent of $x \in \mathbb{R}^{3}$ ).

Corollary 7.23. The first order term of the error map $\eta_{\mathrm{N}} \rightarrow \eta_{\mathrm{B}}$ is given by

$$
\eta_{B}(\psi)=-4 \pi\left(G_{0} \eta_{N} G_{0} \psi\right) \text {, }
$$

for a section $\psi \in \Gamma\left(\mathbb{R}^{3}, \mathrm{E}\right)$.

Proof. Let $\eta_{N}=\varepsilon \eta$, and compare coefficients by powers of $\varepsilon$.

\subsubsection{Bogomolny to Nahm}

Notation 7.24. For brevity we denote the Bogomolny Dirac Operator by just $\mathscr{D}$, instead of $\mathscr{D}_{\mathrm{A}, \mathrm{t}}$ in this section.

Theorem 7.25. Let $(A, \phi)$ satisfy the Bogomolny equations. Then the transformed datum $\left(\nabla_{t}, T_{j}\right)$ satisfies the Nahm equations.

Proof. Let us show the first Nahm equation.

$$
\nabla_{\mathrm{t}} \mathrm{T}_{1}+\left[\mathrm{T}_{2}, \mathrm{~T}_{3}\right]=0
$$

Take a $\psi \in \operatorname{ker} \mathscr{D}^{*}$ (i.e a section of the vector bundle) and calculate

$$
\begin{gathered}
\left(\nabla_{\mathrm{t}} \mathrm{T}_{1}\right)(\psi)=\nabla\left(\mathrm{T}_{1} \psi\right)-\mathrm{T}_{1} \nabla_{\mathrm{t}} \psi=\pi\left(\partial_{\mathrm{t}} \pi\left(\mathfrak{i} x_{1} \psi\right)-\mathfrak{i x} x_{1} \pi\left(\partial_{\mathrm{t}} \psi\right)\right) . \\
{\left[\mathrm{T}_{2}, \mathrm{~T}_{3}\right](\psi)=\pi\left(\mathfrak{i x}_{2} \pi\left(\mathfrak{i} x_{3} \psi\right)-\mathfrak{i} x_{3} \pi\left(\mathfrak{i x _ { 2 }} \psi\right)\right) .}
\end{gathered}
$$

So to show the equation, it suffices to show that

$$
\partial_{\mathrm{t}} \pi\left(i x_{1} \psi\right)-i x_{1} \pi\left(\partial_{t} \psi\right)-i x_{2} \pi\left(i x_{3} \psi\right)-i x_{3} \pi\left(i x_{2} \psi\right) \in \operatorname{ker} \pi
$$

We calculate

$$
\begin{aligned}
& \partial_{\mathrm{t}} \pi\left(i x_{1} \psi\right)-i x_{1} \pi\left(\partial_{\mathrm{t}} \psi\right)=\partial_{\mathrm{t}}\left(i x_{1} \psi-\mathscr{D}\left(\mathscr{D}^{*} \mathscr{D}\right)^{-1} \mathscr{D}^{*}\right)\left(i x_{1} \psi\right) \\
& -i x_{1}\left(\partial_{\mathrm{t}} \psi-\mathscr{D}\left(\mathscr{D}^{*} \mathscr{D}\right)^{-1} \mathscr{D}^{*}\right)\left(\partial_{\mathrm{t}} \psi\right) \\
& =i_{1} \mathscr{D}\left(\mathscr{D}^{*} \mathscr{D}\right)^{-1} \mathscr{D}^{*}\left(\partial_{\mathrm{t}} \psi\right)-\partial_{\mathrm{t}} \mathscr{D}\left(\mathscr{D}^{*} \mathscr{D}\right)^{-1} \mathscr{D}^{*}\left(\mathfrak{i x}_{1} \psi\right) \text {. }
\end{aligned}
$$


Similarly

$$
\begin{aligned}
& i x_{2} \pi\left(i x_{3} \psi\right)-i x_{3} \pi\left(i x_{2} \psi\right)= \\
& \quad i x_{3} \mathscr{D}\left(\mathscr{D}^{*} \mathscr{D}\right)^{-1} \mathscr{D}^{*}\left(i x_{2} \psi\right)-i x_{2} \mathscr{D}\left(\mathscr{D}^{*} \mathscr{D}\right)^{-1} \mathscr{D}^{*}\left(i x_{3} \psi\right) .
\end{aligned}
$$

By a calculation, we see that

$$
\nabla^{\mathrm{A}}\left(i x_{1} \psi\right)=\mathrm{d}\left(i x_{1}\right) \wedge \psi+i x_{1} \nabla^{\mathrm{A}} \psi
$$

hence

$$
\mathscr{D}_{\mathrm{A}}\left(i \chi_{1} \psi\right)=i \mathrm{cl}_{1} \psi+i x_{1} \mathscr{D}_{\mathrm{A}} \psi,
$$

so that

$$
\left[\mathscr{D}_{\mathrm{A}, \mathrm{t}}, i x_{j}\right]=i \mathrm{cl}_{j}
$$

and similarly

$$
\left[\mathscr{D}_{\mathrm{A}, \mathrm{t}}, \partial_{\mathrm{t}}\right]=\mathrm{i} .
$$

Hence

$$
i x_{1} \mathscr{D}\left(\mathscr{D}^{*} \mathscr{D}\right)^{-1} \mathscr{D}^{*}\left(\partial_{\mathrm{t}} \psi\right)=-i \mathrm{cl}_{1}\left(\mathscr{D}^{*} \mathscr{D}\right)^{-1} \mathscr{D}^{*}\left(\partial_{\mathrm{t}} \psi\right) \bmod \operatorname{im} \mathscr{D}
$$

Applying this to all leaves us modulo the image of $\mathscr{D}$ with

$$
\begin{aligned}
& +i\left(\mathscr{D}^{*} \mathscr{D}\right)^{-1} \mathscr{D}^{*}\left(i x_{1} \psi\right)-i \mathrm{cl}_{1}\left(\mathscr{D}^{*} \mathscr{D}\right)^{-1} \mathscr{D}^{*}\left(\partial_{\mathrm{t}} \psi\right) \\
& +i \mathrm{cl}_{2}\left(\mathscr{D}^{*} \mathscr{D}\right)^{-1} \mathscr{D}^{*}\left(i \chi_{3} \psi\right)-i \mathrm{cl}_{3}\left(\mathscr{D}^{*} \mathscr{D}\right)^{-1} \mathscr{D}^{*}\left(i \chi_{2} \psi\right) .
\end{aligned}
$$

Then using the commutators with the adjoint

$$
\left[\mathscr{D}_{\mathrm{A}, \mathrm{t}}^{*}, \partial_{\mathrm{t}}\right]=-\mathfrak{i} \quad\left[\mathscr{D}_{\mathrm{A}, \mathrm{t}}^{*}, i x_{j}\right]=i \mathrm{cl}_{j},
$$

we can permute $\mathscr{D}^{*}$ with the arguments to get by using that $\psi \in \operatorname{ker} \mathscr{D}^{*}$,

$$
\begin{aligned}
& -\left(\mathscr{D}^{*} \mathscr{D}\right)^{-1} \mathrm{cl}_{1} \psi-\mathrm{cl}_{1}\left(\mathscr{D}^{*} \mathscr{D}\right)^{-1} \psi \\
& -\mathrm{cl}_{2}\left(\mathscr{D}^{*} \mathscr{D}\right)^{-1} \mathrm{cl}_{3} \psi+\mathrm{cl}_{3}\left(\mathscr{D}^{*} \mathscr{D}\right)^{-1} \mathrm{cl}_{2} \psi .
\end{aligned}
$$

Note that we have not used that $(A, \phi)$ satisfy the Bogomolny equations yet. Only now do we need the fact, that

$$
\left(\mathscr{D}^{*} \mathscr{D}\right)^{-1}=\mathbb{1}_{\mathbf{S}} \otimes \mathrm{G},
$$

where $G$ is the Greens Operator of the right rand side of the Weitzenböck formula 7.13 for vanishing error $\eta$. The import part is that $\left(\mathscr{D}^{*} \mathscr{D}\right)^{-1}$ commutes with the Clifford multiplication, so that

$$
\left(-\mathrm{cl}_{1}-\mathrm{cl}_{1}-\mathrm{cl}_{2} \mathrm{cl}_{3}+\mathrm{cl}_{3} \mathrm{cl}_{2}\right)\left(\mathscr{D}^{*} \mathscr{D}\right)^{-1} \psi=0,
$$

because $\mathrm{cl}_{2} \mathrm{cl}_{3}=-\mathrm{cl}_{1}$ and $\mathrm{cl}_{3} \mathrm{cl}_{2}=\mathrm{cl}_{1}$. This shows the first Nahm equation. To prove the others, cyclically permute 1,2 and 3 in the proof above. 
Corollary 7.26. For a generalized Bogomolny datum $(A, \phi)$ with error term satisfying $\left\|\mathrm{cl}_{\eta}\right\|<$ $\left\|\mathrm{G}_{\mathrm{O}}\right\|$ (in the operatornorm from $\mathrm{W}^{1,2}$ to $\mathrm{W}^{1,2}$ ), the transformed error term is given by

$$
\psi \mapsto \pi \circ\left(\begin{array}{l}
-\mathrm{Ecl}_{1}-\mathrm{cl}_{1} \mathrm{E}-\mathrm{cl}_{2} \mathrm{Ecl}_{3}+\mathrm{cl}_{3} \mathrm{Ecl}_{2} \\
-\mathrm{Ecl}_{2}-\mathrm{cl}_{2} \mathrm{E}-\mathrm{cl}_{3} \mathrm{Ecl}_{1}+\mathrm{cl}_{1} \mathrm{Ecl} 3 \\
-\mathrm{Ecl}_{3}-\mathrm{cl}_{3} \mathrm{E}-\mathrm{cl}_{1} \mathrm{E} \mathrm{cl}_{2}+\mathrm{cl}_{2} \mathrm{Ecl}_{1}
\end{array}\right) \psi
$$

where

$$
E=\left(\sum_{n=1}^{\infty}\left(G_{0} c_{\eta}\right)^{n}\right) G_{0}
$$

$\mathrm{G}_{0}$ is the Greens operator of

$$
\mathbb{1}_{\mathbb{H}} \otimes\left(\left(\nabla^{\mathrm{A}}\right)^{*} \nabla^{\mathrm{A}}-(\phi-i t \mathbb{1})^{2}\right)
$$

and for a section $\psi \in \Gamma(\mathrm{I}, \mathrm{V})$.

Proof. The proof is identical to the Nahm case.

Corollary 7.27. If $\left\|\mathrm{cl}_{\eta}\right\|<\left\|\mathrm{G}_{0}\right\|^{-1}$ (in the operatornorm from $\mathrm{W}^{1,2}$ to $\mathrm{W}^{1,2}$ ), then the transformed error term $\eta_{\mathrm{Nahm}}$ is bounded on $\mathrm{I}$.

Proof. This follows from the same argument as in the Nahm case, $\left\|G_{0} \psi\right\| \geqslant C t^{2}\|\psi\|$ so that $\left\|\mathrm{G}_{\mathrm{O}}\right\| \leqslant \frac{1}{\mathrm{Ct}^{2}}$, hence $\mathrm{E}$ is bounded.

Corollary 7.28. The first order term of the error map $\eta_{\mathrm{B}} \rightarrow \eta_{\mathrm{N}}$ is given by

$$
\eta_{N}(\psi)=4 \pi\left(G_{0} \eta_{B} G_{0} \psi\right)
$$

for a section $\psi \in \Gamma(\mathrm{I}, \mathrm{V})$.

Proof. Let $\eta_{\mathrm{B}}=\varepsilon \eta$, and compare coefficients by powers of $\varepsilon$.

\subsection{Boundary Conditions}

The idea of the proof of the boundary conditions is identical for both directions. First an approximation operator is introduced that has the same boundary behavior as the Dirac operator. Then it is shown that the kernel of this new operator is close to the original one, in the sense that the boundary behavior of the elements in the kernel is sufficiently the same. Finally explicit solutions in the kernel of the approximating operator are given and it is shown that those have the correct boundary behavior.

We have to modify these proofs in two steps. We define the same approximating operator but have to show that the additional term $\mathrm{cl}_{\eta}$ in the Weitzenböck formulas still allow to show that the elements in the kernel are close. For this only need the asymptotic of the Weitzenböck formulas, which is unchanged by the modifications. This is justified in equations 7.126 and 7.147. 
The second step is that we need to make sure that the solutions of the approximated operator still have the correct boundary behavior (this uses satisfaction of the equations). Again it turns out that we merely need an asymptotic satisfaction of the equations, i.e. the transformed error has to be $\eta \in \mathcal{O}\left(r^{-2}\right)$ in the Bogomolny equations and $\eta \in \mathcal{O}\left(\frac{1}{t+1}\right)$ for $t \rightarrow-1$ and $\eta \in \mathcal{O}\left(\frac{1}{t-1}\right)$ for $t \rightarrow 1$ in the Nahm equations.

Since these poofs use a vast amount of details, we will not reproduce them completely here but rather give a overview of the important steps and show where adjustments have to be made.

\section{7·4.1 Nahm to Bogomolny}

We closely follow $[\overline{\mathrm{Hit} 83}]$ in this section.

Theorem 7.29. Let $\left(T_{0}, T_{1}, T_{2}, T_{3}\right)$ be an approximate solution to the Nahm equations of charge $k \in \mathbb{N}$, so that the transformed error term satisfies $\tilde{\eta} \in \mathcal{O}\left(\mathrm{r}^{-2}\right)$. Then the transformed datum $(A, \phi)$ satisfies the boundary conditions $B_{k}$.

Proof. We use C as a generic constant in this proof. As $\mathbf{S U}(2)$-representations we have the following decomposition.

$$
H \otimes V \cong S^{1} \otimes S^{k-1} \cong S^{k} \oplus S^{k-2} .
$$

Close to the boundary,

$$
\mathrm{T}_{j}=\frac{\rho_{j}}{1-\mathrm{t}}+\mathrm{b}(\mathrm{t}),
$$

with $\mathrm{b}$ smooth. The action of $\mathrm{T}=\sum_{j} \mathrm{cl}_{j} \otimes \rho_{j}$ can be understood via the Casimir operator to be multiplication by $\frac{1-k}{2}$ on $S^{k}$ and $\frac{-1-k}{2}$ on $S^{k-2}$. Let us approximate $\mathscr{D}_{x}$ by

$$
\mathscr{D}_{\mathrm{x}}^{0}=\mathbb{1}_{\mathbb{H}} \otimes \partial_{\mathrm{t}}+\left(\frac{1}{\mathrm{t}+1}+\frac{1}{\mathrm{t}-1}\right) \mathrm{T}-\mathrm{i} \sum_{j=1}^{3} \mathrm{cl}_{j} \otimes \mathrm{x}_{\mathrm{j}} \mathbb{1}_{\mathrm{V}} .
$$

Then $\mathscr{D}_{x}-\mathscr{D}_{x}^{0}=\mathrm{A}$ is a smooth operator on $\overline{\mathrm{I}}$ independent of $x \in \mathbb{R}^{3}$,

$$
A=\mathbb{1}_{\mathbb{H}} \otimes T_{0}+\sum_{j=1}^{3} \mathrm{cl}_{j} \otimes\left(T_{j}-\frac{\rho_{j}}{1+t}-\frac{\rho_{j}}{1-t}\right),
$$

so the index of $\mathscr{D}_{x}^{0}$ and $\mathscr{D}_{x}$ are equal. We can give explicit solutions in $\operatorname{ker}_{\mathrm{L}^{2}}\left(\mathscr{D}_{x}^{0}\right)^{*}$

$$
g_{ \pm}(t)=\left(t^{2}-1\right)^{\frac{k-1}{2}} e^{ \pm r(t-1)} v_{ \pm} .
$$

To explain this, fix $x \in \mathbb{R}^{3}$ for a second up to a scalar, i.e. $x=r u$ for a unitary quaternion $u \in \mathbb{R}^{3} \cong \operatorname{Im} \mathbb{H}$. Identifying $\mathfrak{s u}(2) \cong \operatorname{Im} \mathbb{H}$, u generates a copy $\mathbf{U}(1) \subset \mathbf{S U}(2)$ which decomposes the representations $S^{k}$ and $S^{k-2}$ into one-dimensional weight spaces,

$$
\begin{aligned}
S^{k} & =V_{k} \oplus V_{k-2} \oplus \cdots \oplus V_{-k} \\
S^{k-2} & =V_{k-2} \oplus V_{k-4} \oplus \cdots \oplus V_{-k+2}
\end{aligned}
$$


Now let $v_{ \pm}$be the highest (lowest) weight of $S^{k}$, which implies that it is fixed in $\mathbb{H} \otimes V$ by the action of $-i r \sum_{j} c_{j} \otimes u_{j} \mathbb{1}_{V}$. Since $\left(-i \sum_{j} \mathrm{cl}_{j} \otimes u_{j} \mathbb{1}_{V}\right)^{2}=1$, we see that

$$
\left(\sum_{j=1}^{3} \mathrm{cl}_{j} \otimes \mathrm{x}_{j} \mathbb{1}_{V}\right) v_{ \pm}= \pm r v_{ \pm},
$$

for $r=\|x\|$. Now we can see that

$$
\left(\mathscr{D}_{x}^{0}\right)^{*} g_{ \pm}(t)=\left(-\frac{2 t}{t^{2}-1} \mp r+\frac{1}{t+1}+\frac{1}{t-1}+ \pm r\right) g_{ \pm}(t)=0 .
$$

For big enough $r$ there is a $c_{j}>0$ such that $\left\|T_{j}-i x_{j}\right\| \geqslant c_{j}\left\|x_{j}\right\|$, which together with the Weitzenböck formula implies that for $\|x\| \geqslant r$,

$$
\left\langle\mathscr{D}_{x} \psi, \mathscr{D}_{x} \psi\right\rangle \geqslant\left(\mathrm{r}^{2}-\left\|\mathrm{cl}_{\eta}\right\|\right)\|\psi\|^{2} \geqslant \mathrm{Cr}^{2}\|\psi\|^{2},
$$

for $C>0$ and $r$ big enough, where we have used that $\eta$ is independent of $x \in \mathbb{R}^{3}$. This implies

$$
\left\langle\mathscr{D}_{x}^{0} \psi, \mathscr{D}_{x}^{0} \psi\right\rangle=\left\langle\left(\mathscr{D}_{x}-A\right) \psi,\left(\mathscr{D}_{x}-A\right) \psi\right\rangle \geqslant C^{2} r^{2}\|\psi\|^{2},
$$

if $\|x\| \geqslant r$ for $r$ big enough. Hence, $\operatorname{ker} \mathscr{D}_{x}^{0}=0$ for $\|x\|$ big enough. Let $G_{0}$ be the Greens Operator of $\mathscr{D}_{x}^{0}$, i.e.

$$
\mathscr{D}_{x}^{0} \mathrm{G}_{0}=\mathbb{1}-\pi_{0}, \quad \mathrm{G}_{0} \mathscr{D}_{x}^{0}=\mathbb{1}
$$

with $\pi_{0}$ the orthogonal projection onto $\operatorname{ker}\left(\left(\mathscr{D}_{x}^{0}\right)^{*}\right)$. Hence we have

$$
\|\psi\|^{2}-\left\|\pi_{0} \psi\right\|^{2} \geqslant \mathrm{Cr}^{2}\left\|\mathrm{G}_{0} \psi\right\|^{2},
$$

which implies that $\left\|\mathrm{G}_{0}\right\| \in \mathcal{O}\left(\mathrm{r}^{-1}\right)$, and then $\left\|\mathrm{G}_{0}^{*}\right\| \in \mathcal{O}\left(\mathrm{r}^{-1}\right)$. If $\psi \in \operatorname{ker} \mathscr{D}^{*}$, then

$$
\psi-\pi_{0} \psi=G_{0}^{*} \mathscr{D}_{0}^{*} \psi=G_{0}^{*}\left(\mathscr{D}^{*}-A^{*}\right) \psi=-G_{0}^{*} A^{*} \psi,
$$

so that $\left\|\psi-\pi_{0} \psi\right\| \leqslant \frac{\tilde{\mathrm{C}}}{\mathrm{r}}\|\psi\|$. This shows that the elements in kernel of $\mathscr{D}^{*}$ can be approximated to order $r^{-1}$ by elements in the kernel of $\mathscr{D}_{0}^{*}$.

[Hit83] now goes on to shows that the solutions of $\mathscr{D}_{0}^{*}$, which can be expressed by $g_{ \pm}$, satisfy the boundary conditions which implies the satisfaction of the boundary conditions of the elements in the kernel of $\mathscr{D}^{*}$. Notice that our $\mathscr{D}_{0}$ is identical to the one in [Hit83]. The only part where the equations come into play is shortly after equation [Hit83, Equation (2.13)], where $\left\|F^{A}\right\| \in \mathcal{O}\left(r^{-2}\right)$ needs to imply $\left\|d_{A} \phi\right\| \in \mathcal{O}\left(r^{-2}\right)$, which is true because of the assumption $\tilde{\eta} \in \mathcal{O}\left(r^{-2}\right)$ on the transformed error term.

Corollary 7.30. If $\left\|\mathrm{cl}_{\eta}\right\|<\left\|\mathrm{G}_{0}\right\|^{-1}$ and $\mathscr{D}_{x}^{*} \mathscr{D}_{\mathrm{x}}$ is a positive operator, then the extended Nahm transform from Nahm to Bogomolny is well defined.

Proof. This follows by Theorem 7.29 and Corollary 7.21 .

\footnotetext{
${ }^{1} \mathscr{D}_{x}$ is called $\Delta(x)$ in Hitchin's notation, $\mathscr{D}_{x}^{0}$ is denoted by $\Delta_{0}(x)$ and defined shortly after [Hit83. Theorem 2.8]. The relation between our definitions is $i \mathscr{D}_{x}=\Delta(x)$. Note also that Hitchin defines $\Delta(x)$ for $x \in \mathbb{R}^{4}$, but restricts it to $x \in \mathbb{R}^{3}$ after $[$ Hit83. Equation (2.10)]. Finally note that Hitchin uses the interval $\mathrm{I}=(0,2)$.
} 


\subsubsection{Bogomolny to Nahm}

We follow closely the description in Nakajima's paper [Nak91]. Similarly to this work $\mathrm{C}$ is used as a generic constant.

Theorem 7.31. Let $(A, \phi)$ be an approximate solution to the Nahm equations of charge $k \in \mathbb{N}$ and let the transformed error term satisfy $\tilde{\eta} \in \mathcal{O}\left(\frac{1}{\mathrm{t}+1}\right)$ for $\mathrm{t} \rightarrow-1$ and $\tilde{\eta} \in \mathcal{O}\left(\frac{1}{\mathrm{t}-1}\right)$ for $\mathrm{t} \rightarrow 1$. Then the transformed datum satisfies the boundary conditions $\mathrm{NC}_{\mathrm{k}}$.

Proof. Define

$$
\mathscr{D}_{\mathrm{A}, \mathrm{t}}=\mathscr{D}_{\mathrm{A}}+(\phi-i \mathrm{t}): \Gamma(\mathbf{S} \otimes \mathrm{E}) \rightarrow \Gamma(\mathbf{S} \otimes \mathrm{E})
$$

and use the index theorem of Callias (see Theorem 7.17) to see that this operator has index $k$. The Weitzenböck formula shows that

$$
\mathscr{D}_{\mathrm{A}, \mathrm{t}}^{*} \mathscr{D}_{\mathrm{A}, \mathrm{t}}=\nabla^{*} \nabla-\mathbb{1}_{\mathrm{S}} \otimes(\phi-i \mathrm{t} \mathbb{1})^{2}-\mathrm{cl}_{\eta},
$$

which is a positive operator if $\left\|\mathrm{cl}_{\eta}\right\|$ is small enough in the operator norm from $W^{1,2}$ to $\mathrm{L}^{2}$. Hence $\operatorname{ker}_{\mathrm{L}^{2}} \mathscr{D}_{\mathrm{A}, \mathrm{t}}^{*}$ is $\mathrm{k}$-dimensional. This defines a subbundle of the trivial vector bundle

$$
\mathrm{V} \subset \underline{\mathrm{L}^{2}\left(\mathbb{R}^{3}, \mathrm{~S} \otimes \mathrm{E}\right)} \rightarrow \mathrm{I}
$$

by letting $\mathrm{V}_{\mathrm{t}}=\operatorname{ker}_{\mathrm{L}^{2}} \mathscr{D}_{\mathrm{A}, \mathrm{t}}^{*}$. Nakajima shows [Nak91] that the boundary conditions assure that we can find an assymptotic gauge that the bundle $E$ decomposes into $L \oplus L^{*}$ the sum of two line bundles (the eigenspaces of $\phi$ for eigenvalues $i$ and $-i$ ) with $L=\mathcal{O}(k)(k \in \mathbb{N}$ from the boundary conditions). He goes on showing, that in this gauge

$$
A=\left(\begin{array}{cc}
A_{\infty}^{*} & 0 \\
0 & A_{\infty}
\end{array}\right)+\mathcal{O}\left(r^{-2}\right),
$$

and using this for the operator implies that

$$
\mathscr{D}_{\mathrm{A}, \mathrm{t}}^{*}=\left(\begin{array}{cc}
\mathrm{B}_{1} & 0 \\
0 & \mathrm{~B}_{2}
\end{array}\right)+\mathcal{O}\left(\mathrm{r}^{-2}\right),
$$

where

$$
\begin{aligned}
& B_{1}=\left(\begin{array}{cc}
i\left(\partial_{\mathrm{r}}+\mathrm{t}-1+\frac{\mathrm{k}+2}{2 \mathrm{r}}\right) & \frac{1}{\mathrm{r}} \mathscr{D}_{\mathrm{A}_{\infty}^{*}}^{-} \\
\frac{1}{\mathrm{r}} \mathscr{D}_{\mathrm{A}_{\infty}^{*}}^{*} & -i\left(\partial_{\mathrm{r}}-\mathrm{t}+1-\frac{k-2}{2 r}\right)
\end{array}\right) \\
& \mathrm{B}_{2}=\left(\begin{array}{cc}
i\left(\partial_{\mathrm{r}}+\mathrm{t}+1-\frac{\mathrm{k}-2}{2 \mathrm{r}}\right) & \frac{1}{\mathrm{r}} \mathscr{D}_{\mathrm{A}_{\infty}}^{-} \\
\frac{1}{\mathrm{r}} \mathscr{D}_{\mathrm{A}_{\infty}}^{+} & -i\left(\partial_{\mathrm{r}}-\mathrm{t}-1+\frac{k+2}{2 r}\right)
\end{array}\right),
\end{aligned}
$$

see page 4 of [Nak91]. We take $\mathscr{D}_{A, t}^{0}=\operatorname{diag}\left(\mathrm{B}_{1}, \mathrm{~B}_{2}\right)$ as the approximating operator, but we need to explain the notation first. Notice that the Spin bundle of $\mathbb{R}^{3} \backslash\{0\}=\mathbb{R}^{+} \times \mathbb{S}^{2}$ 
is isomorphic to the Spin bundle of $S^{2}$, which decomposes into $\mathbf{S}^{+}$and $\mathbf{S}^{-}$and $\mathscr{D}_{\mathbf{A}_{\infty}}^{ \pm}$ are the Dirac operators of $S^{2}$ twisted by $A_{\infty}$. $A_{\infty}$ is the connection induced by $A$ on the line bundle L. The matrix acts on the decomposition

$$
\begin{aligned}
\mathrm{S} \otimes \mathrm{E} & =\mathrm{L}^{*} \otimes \mathrm{S}^{+} \oplus \mathrm{L}^{*} \otimes \mathrm{S}^{-} \oplus \mathrm{L} \otimes \mathrm{S}^{+} \oplus \mathrm{L} \oplus \mathrm{S}^{-} \\
& =\mathcal{O}(-\mathrm{k}-1) \oplus \mathcal{O}(-\mathrm{k}+1) \oplus \mathcal{O}(\mathrm{k}-1) \oplus \mathcal{O}(\mathrm{k}+1) .
\end{aligned}
$$

This time we won't be able to give a explicit basis in $\operatorname{ker}\left(\mathscr{D}_{A, t}^{0}\right)^{*}$, but we can give a approximation in the following sense. Focusing on the boundary conditions at $t \rightarrow-1$, we define a k-dimensional space of functions close to $\operatorname{ker}\left(\mathscr{D}_{\mathrm{A}, \mathrm{t}}^{0}\right)^{*}$ by

$$
\psi(r, \theta)=\chi(r) e^{-(t+1) r} r^{\frac{k-2}{2}}\left(\begin{array}{c}
0 \\
0 \\
f(\theta) \\
0
\end{array}\right)
$$

for $f \in H^{\mathcal{O}}\left(\mathbb{C P}^{1}, \mathcal{O}(k-1)\right)$ (recall that $\mathcal{O}(k-1)$ has a $k$-dimensional space of holomorphic sections), and $\chi(r)$ a cut-off function which is 0 on $[0, R]$ and 1 on $[R+1, \infty)$ for some $R>0$. Here $r$ is the coordinate on $\mathbb{R}^{+}$and $\theta$ on $S^{2}$. Direct calculations show that

$$
\left|\mathscr{D}_{\mathrm{A}, \mathrm{t}}^{*} \psi\right|_{\mathrm{S} \otimes \mathrm{E}} \leqslant \mathrm{C} e^{-(\mathrm{t}+1) \mathrm{R}}(\mathrm{R}+1)^{\frac{\mathrm{k}-2}{2}}
$$

for $C$ independent of $R$ and $t$. In this sense it is an approximate solution to $\operatorname{ker}\left(\left(\mathscr{D}_{\AA, t}^{0}\right)^{*}\right)$ for large R. A solution $\varphi$ of the equation

$$
\mathscr{D}_{\mathrm{A}, \mathrm{t}}^{*} \mathscr{D}_{\mathrm{A}, \mathrm{t}} \varphi=\mathbb{1}_{\mathrm{S}} \otimes\left(\nabla_{\mathrm{A}}^{*} \nabla_{\mathrm{A}}-(\phi-i \mathrm{t})^{2}-\mathrm{cl}_{\eta}\right) \varphi=\mathscr{D}_{\mathrm{A}, \mathrm{t}}^{*} \psi
$$

then gives an element in $\operatorname{ker} \mathscr{D}_{A, t}^{*}$ as $\psi-\mathscr{D}_{\mathrm{A}, \mathrm{t}} \varphi$. We will show, that the boundary behavior of this element is dominated by $\psi$, but first we argue that such a $\varphi$ exists (uniquely). The equation

$$
\mathbb{1}_{S} \otimes\left(\nabla_{A}^{*} \nabla_{A}-(\phi-i t)^{2}-\mathrm{cl}_{\eta}\right) \varphi-\mathscr{D}_{A, t}^{*} \psi=0
$$

is the Euler Lagrange equation of the functional

$$
S(\varphi)=\frac{1}{2}\left\|\nabla_{A} \varphi\right\|_{\mathrm{L}^{2}}^{2}+\frac{1}{2}\|(\phi-i \mathrm{t}) \varphi\|_{\mathrm{L}^{2}}^{2}-\left\langle\varphi, \mathrm{cl}_{\eta} \varphi\right\rangle_{\mathrm{L}^{2}}-\left\langle\varphi, \mathscr{D}_{\mathrm{A}, \mathrm{t}}^{*} \psi\right\rangle_{\mathrm{L}^{2}} .
$$

This is a strictly convex function, which is also differentiable and coercive (see [JT80, Proposition IV.4.1]). By methods of variational calculus (see e.g. [JT80, Proposition VI.8.5]), it has a unique minimum $S(\varphi) \leqslant S(0)=0$. Therefore

$$
\left\|\mathscr{D}_{\mathrm{A}, \mathrm{t}} \varphi\right\|_{\mathrm{L}^{2}}^{2}=\left\|\nabla_{\mathrm{A}} \varphi\right\|_{\mathrm{L}^{2}}^{2}+\|(\phi-i \mathrm{t}) \varphi\|_{\mathrm{L}^{2}}^{2}-2\left\langle\varphi, \mathrm{cl}_{\eta} \varphi\right\rangle_{\mathrm{L}^{2}} \leqslant 2\left\langle\varphi, \mathscr{D}_{\mathrm{A}, \mathrm{t}}^{*} \psi\right\rangle_{\mathrm{L}^{2}} .
$$


For $t$ sufficiently close to -1 , we can estimate all these terms from below against the $\mathrm{L}^{2}$ norm of $\varphi$, i.e.

$$
\|(1+\mathrm{t}) \varphi\|_{\mathrm{L}^{2}}^{2} \leqslant \mathrm{C}\left(\left\|\nabla_{\mathrm{A}} \varphi\right\|_{\mathrm{L}^{2}}^{2}+\|(\phi-i \mathrm{t}) \varphi\|_{\mathrm{L}^{2}}^{2}-2\left\langle\varphi, \mathrm{cl}_{\eta} \varphi\right\rangle\right)=\mathrm{C}\left\|\mathscr{D}_{\mathrm{A}, \mathrm{t}} \varphi\right\|_{\mathrm{L}^{2}}^{2},
$$

for details on how to estimate the first two terms see [Nak91. Equations (2.4) and (2.5)]. But then we can restrict $t$ to a smaller neighborhood of -1 , so that it is satisfied also with our error term $\left\langle\mathrm{cl}_{\eta} \varphi, \varphi\right\rangle \leqslant\left\|\mathrm{cl}_{\eta}\right\|\|\varphi\|^{2}$ (independent of $\varphi$ ). Once we have established this equations, we can apply this to equation (7.146), to get

$$
\left\|\mathscr{D}_{\mathrm{A}, \mathrm{t}} \varphi\right\|_{\mathrm{L}^{2}}^{4} \stackrel{7.146}{\leqslant} 4\|\varphi\|_{\mathrm{L}^{2}}^{2}\left\|\mathscr{D}_{\mathrm{A}, \mathrm{t}}^{*} \psi\right\|_{\mathrm{L}^{2}}^{2} \stackrel{7.147}{\leqslant} \frac{1}{(1+\mathrm{t})^{2}} \mathrm{C}\left\|\mathscr{D}_{\mathrm{A}, \mathrm{t}} \varphi\right\|_{\mathrm{L}^{2}}^{2}\left\|\mathscr{D}_{\mathrm{A}, \mathrm{t}}^{*} \psi\right\|_{\mathrm{L}^{2}}^{2},
$$

or $\left\|\mathscr{D}_{\mathrm{A}, \mathrm{t}} \varphi\right\|_{\mathrm{L}^{2}} \leqslant \frac{1}{1+\mathrm{t}} \mathrm{C}\left\|\mathscr{D}_{\mathrm{A}, \mathrm{t}}^{*} \psi\right\|_{\mathrm{L}^{2}}$. From a direct calculation, we see that

$$
\left\|\mathscr{D}_{\mathrm{A}, \mathrm{t}}^{*} \psi\right\|_{\mathrm{L}^{2}}=\mathrm{C}(1+\mathrm{t})^{2}\|\psi\|_{\mathrm{L}^{2}},
$$

which shows that $\left\|\mathscr{D}_{\mathrm{A}, \mathrm{t}} \varphi\right\|_{\mathrm{L}^{2}} \leqslant \mathrm{C}(1+\mathrm{t})\|\psi\|_{\mathrm{L}^{2}}$, so the boundary behavior close to $t \rightarrow-1$ is dominated by $\psi$. It remains to show, that the action of $T_{i}$ on $\psi$ has the correct boundary conditions. This is shown in [Nak91, Lemma 2.6] where Nakajima shows that the action of $T_{i}$ on $f \in \mathrm{H}^{0}\left(\mathbb{C P}^{1}, \mathcal{O}(k-1)\right)$ is a multiple of a $k$-dimensional irreducible $\mathfrak{s u}(2)$ representation. The multiplicity is then fixed by satisfaction of the Nahm equations, where we need that the transformed error $\tilde{\eta}$ is in $\mathcal{O}\left(\frac{1}{t-1}\right)$ for $t \rightarrow 1$ and in $\mathcal{O}\left(\frac{1}{\mathfrak{t}+1}\right)$ for $t \rightarrow-1$, because then for $T_{j}=\frac{\rho_{j}}{1+t}+b_{j}$ with $b_{j} \in \mathfrak{C}^{\infty}(\bar{I}, \mathfrak{g})(j \in\{1,2,3\})$

$$
\nabla_{\mathrm{t}} \mathrm{T}_{\mathrm{i}}+\frac{1}{2} \sum_{j, k=1}^{3} \varepsilon_{i j k}\left[\mathrm{~T}_{j}, \mathrm{~T}_{k}\right]=\frac{1}{(\mathrm{t}+1)^{2}}\left(\rho_{i}+\frac{1}{2} \sum_{j, k=1}^{3}\left[\rho_{j}, \rho_{k}\right]\right)+\mathcal{O}\left(\frac{1}{\mathrm{t}+1}\right)
$$

for $t \rightarrow-1$ and similarly for $t \rightarrow 1$.

Corollary 7.32. If $\left\|\mathrm{cl}_{\eta}\right\|<\left\|\mathrm{G}_{0}\right\|^{-1}$ and $\mathscr{D}_{\mathrm{A}, \mathrm{t}}^{*} \mathscr{D}_{\mathrm{A}, \mathrm{t}}$ is a positive operator, then the extended Nahm transform form Bogomolny to Nahm is well defined.

Proof. This follows by Theorem 7·31 and Corollary $7 \cdot 26$.

\subsection{Behavior with Respect to Conformal Maps}

Recall, that the anti self-duality equations are conformally invariant.

Proposition 7.33. The ASD equations are conformally invariant. Let

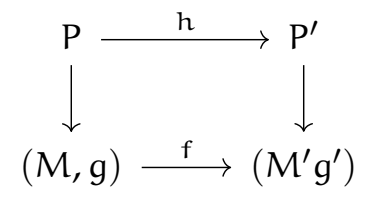


with $\mathrm{h}$ a $\mathrm{G}$-equivariant map and $\mathrm{f}$ an orientation preserving conformal map. Then $\mathrm{A} \in \mathrm{C}\left(\mathrm{P}^{\prime}\right)$ asd implies $\mathrm{h}^{*} \mathrm{~A} \in \mathcal{C}(\mathrm{P})_{\text {asd }}$.

Proof. See e.g. [Bauog, Satz 7.7].

Remark 7.34. Note that if $f^{*} g^{\prime}=u^{2} g$ for a function $u$, then

$$
\left.\star_{g}\right|_{\Lambda^{k}}=\left.u^{n-2 k} \star_{u^{2} g}\right|_{\Lambda^{k}},
$$

so in particular for $n=4, k=2$ we have $\left.\star_{g}\right|_{\wedge^{2}}=\left.\star_{u^{2} g}\right|_{\wedge^{2}}$. By Liouville's theorem the conformal maps on $\mathbb{R}^{4}$ can be decomposed into

- homothetic transformations

- isometries

- special transformations (reflections and an inversion on a sphere)

It seems natural to expect that Bogomolny monopoles and the Nahm equations are well behaved under these transformations respecting the decomposition of the dimensional reduction. We will show that this is true and also that the Nahm transform is equivariant with respect to those. It turns out that we already discussed the isometries, which are a combination of the $\mathbb{R}^{3}$ and $\mathbf{S O}(3)$ actions on the solutions of Nahm and Bogomolny. Homothetic transformations are given by

$$
f_{\varepsilon}: \mathbb{R}^{4} \rightarrow \mathbb{R}^{4}, x \mapsto \varepsilon x,
$$

and the special transformations do not respect the decomposition of the dimensional reductions.

Proposition 7.35. When we apply the homothety $\mathrm{f}$ to the Nahm equations, the interval gets scaled to $\varepsilon \mathrm{I}$. We have $\mathcal{M}^{\mathrm{Nahm}} \cong \mathcal{M}_{\varepsilon}^{\mathrm{Nahm}}$

Proof. Define

$$
\mathrm{T}_{i}^{\varepsilon}(\mathrm{t})=\frac{1}{\varepsilon} \mathrm{T}_{\mathrm{i}}\left(\frac{\mathrm{t}}{\varepsilon}\right)
$$

Then we calculate

$$
\begin{aligned}
& \left(\dot{\mathrm{T}}_{i}^{\varepsilon}+\left[\mathrm{T}_{0}^{\varepsilon}, \mathrm{T}_{i}^{\varepsilon}\right]+\frac{1}{2} \sum_{j k} \varepsilon_{i j k}\left[\mathrm{~T}_{i}^{\varepsilon}, \mathrm{T}_{j}^{\varepsilon}\right]\right)(\varepsilon t)= \\
& \frac{1}{\varepsilon^{2}}\left(\dot{\mathrm{T}}_{i}+\left[\mathrm{T}_{0}, \mathrm{~T}_{i}\right]+\frac{1}{2} \sum_{j k} \varepsilon_{i j k}\left[\mathrm{~T}_{i}, \mathrm{~T}_{j}\right]\right)(\mathrm{t})=0 .
\end{aligned}
$$

This gives a map from solutions to the Nahm equations on I to solutions on $\mathrm{I}^{\varepsilon}=$ $\varepsilon \mathrm{I}=(-\varepsilon, \varepsilon)$. 
Mapping the gauge transformations, via

$$
\mathcal{G}_{\varepsilon}^{\mathrm{Nahm}} \rightarrow \mathcal{G}^{\mathrm{Nahm}}, \quad g_{\varepsilon}(t)=g\left(\frac{1}{\varepsilon} \mathrm{t}\right),
$$

shows that

$$
g_{\varepsilon} T_{0}^{\varepsilon}(t)=g_{\varepsilon}(t) \frac{1}{\varepsilon} T_{0}\left(\frac{t}{\varepsilon}\right) g_{\varepsilon}{ }^{-1}(t)-\frac{1}{\varepsilon} \dot{g}_{\varepsilon}(t) g_{\varepsilon}{ }^{-1}(t)=\left(g T_{0}\right)^{\varepsilon}(t)
$$

and similarly

$$
g_{\varepsilon} T_{i}^{\varepsilon}=\left(g T_{i}\right)^{\varepsilon} .
$$

This shoes that the map projects down onto a map of the quotients and we have shown the isomorphism. (The inverse is $T_{i}(x)=\varepsilon T_{i}^{\varepsilon}(\varepsilon x)$ )).

Proposition 7.36. When we apply the homothety $f$ to the Bogomolny equations, $\mathbb{R}^{3}$ gets stretched by $\varepsilon$. We have $\mathcal{M}_{\varepsilon}^{\text {Bog }} \cong \mathcal{M}^{\text {Bog }}$

Proof. The argument is similar with

$$
A_{x}^{\varepsilon}=f_{\frac{1}{\varepsilon}}^{*} A_{x}=A_{\frac{1}{\varepsilon} x} \circ d f_{\frac{1}{\varepsilon}}=\frac{1}{\varepsilon} A_{\frac{1}{\varepsilon} x} .
$$

and

$$
\varphi^{\varepsilon}(x)=\left(f_{\frac{1}{\varepsilon}}^{*} \varphi\right)(x)=\varphi\left(\frac{1}{\varepsilon} x\right) .
$$

Then this implies

$$
\begin{aligned}
f_{\frac{1}{\varepsilon}}^{*} F_{x}^{A} & =\frac{1}{\varepsilon^{2}} F_{\frac{1}{\varepsilon} x}^{A} \\
f_{\frac{1}{\varepsilon}}^{*} d_{A} \varphi_{\chi} & =\frac{1}{\varepsilon} d_{A} \varphi_{\frac{1}{\varepsilon} x}
\end{aligned}
$$

and in particular

$$
\mathrm{F}_{\chi}^{\mathrm{A}^{\varepsilon}}=\frac{1}{\varepsilon^{2}} \mathrm{~F}_{\frac{1}{\varepsilon} \chi}^{\mathrm{A}}=\frac{1}{\mathcal{\varepsilon}^{2}} \star_{g} \mathrm{~d}_{\mathrm{A}} \varphi_{\frac{1}{\varepsilon} \chi}=\star_{\frac{1}{\varepsilon^{2}} g} \mathrm{~d}_{\mathcal{A}} \varphi_{\chi}^{\varepsilon},
$$

so it maps solutions to solutions.

The boundary conditions are satisfied trivially, since

$$
\lim _{r \rightarrow \infty}\left\|\phi^{\varepsilon}\right\|=\lim _{r \rightarrow \infty}\|\phi\|=1 .
$$

With gauging

$$
\mathcal{G}_{\varepsilon}^{\text {Bog }} \rightarrow \mathcal{G}^{\text {Bog }}, \quad g_{\varepsilon}=g\left(\frac{1}{\varepsilon} x\right) .
$$

we have

$$
g_{\varepsilon} A^{\varepsilon}=(g A)^{\varepsilon}, \quad g_{\varepsilon} \varphi=(g \varphi)^{\varepsilon} .
$$

The boundary condition remains satisfied because

$$
\lim _{r \rightarrow \infty} g_{\varepsilon}(x, y, z)=\lim _{r \rightarrow \infty} g(x, y, z)=1 .
$$


Proposition 7.37. The Nahm transform maps

$$
\mathcal{M}_{\mathrm{Nahm}}^{\varepsilon} \rightarrow \mathcal{M}_{\mathrm{Bog}}^{\varepsilon},
$$

i.e. it commutes with stretching.

Proof. Recall the operator

$$
\begin{gathered}
\mathscr{D}_{x}=\mathbb{1}_{\mathrm{C}^{2}} \otimes \nabla_{\mathrm{t}}+\left(\sum_{j=1}^{3} \sigma_{j} \otimes \mathrm{T}_{j}-i x_{j} \sigma_{j} \otimes \mathbb{1}_{\mathrm{V}}\right) \\
: \mathrm{W}_{\mathrm{O}}^{1,2}\left(\mathrm{I}, \mathrm{C}^{2} \otimes \mathrm{V}\right) \rightarrow \mathrm{L}^{2}\left(\mathrm{I}, \mathbb{C}^{2} \otimes \mathrm{V}\right)
\end{gathered}
$$

where $V$ is the $\mathbf{U}(k)$ vector bundle of the Nahm equations. Also recall the definition of the 2-dimensional sub bundle as

$$
\mathrm{E}_{\mathrm{x}}=\operatorname{ker} \mathscr{D}_{x}^{*} \subset \underline{\mathrm{L}^{2}\left(\mathrm{I}, \mathrm{C}^{2} \otimes \mathrm{V}\right)} x .
$$

We see that

$$
\mathscr{D}_{x}^{\mathcal{\varepsilon}}=\varepsilon \mathscr{D}_{\frac{\mathrm{x}}{\varepsilon}} .
$$

This implies that

$$
\operatorname{ker}\left(\left(\mathscr{D}_{x}^{\varepsilon}\right)^{*}\right)=\operatorname{ker} \mathscr{D}_{\frac{x}{\varepsilon}}^{*}
$$

i.e.

$$
E^{\varepsilon}=f_{\frac{1}{\varepsilon}}^{*} E .
$$

Further

$$
p_{x}^{\varepsilon}=p_{\frac{x}{\varepsilon}}
$$

which implies that

$$
\left(d_{A}^{\varepsilon}\right)_{\chi}=d_{A_{\chi}^{\varepsilon}}=p_{\frac{x}{\varepsilon}} \frac{1}{\mathcal{E}} d=\frac{1}{\mathcal{E}} p_{\frac{\chi}{\varepsilon}} d=\frac{1}{\mathcal{E}}\left(d_{A}\right)_{\frac{\chi}{\varepsilon}} .
$$

and similarly

$$
\phi^{\varepsilon}(x)=p_{\frac{x}{\varepsilon}} i \frac{t}{\varepsilon}=\phi\left(\frac{x}{\varepsilon}\right),
$$

where we used the identification

$$
\mathrm{L}^{2}\left(\mathrm{I}^{\varepsilon}, \mathrm{V}\right) \stackrel{\cdot \frac{1}{\varepsilon}}{\rightarrow} \mathrm{L}^{2}(\mathrm{I}, \mathrm{V}),
$$

that swallows a factor $\frac{1}{\varepsilon}$.

For the reverse notice that the inverse of an equivariant map is automatically equivariant.

Proposition 7.38. The Nahm transform is equivariant with respect to the $\mathbb{R}^{3}$-actions. 
Proof. Again looking at the operator

$$
\mathscr{D}_{x}=\mathbb{1}_{\mathrm{C}^{2}} \otimes \nabla_{\mathrm{t}}+\left(\sum_{j=1}^{3} \sigma_{j} \otimes \mathrm{T}_{j}-i x_{j} \sigma_{j} \otimes \mathbb{1}_{V}\right),
$$

we notice that if we act by $y \in \mathbb{R}^{3}$ on $T_{j}$ and use these matrices to define the operator, then

$$
\mathscr{D}_{x}^{y}=\mathbb{1}_{C} \otimes \nabla_{t}+\left(\sum_{j=1}^{3} \sigma_{j} \otimes T_{j}-i\left(x_{j}-y_{j}\right) \sigma_{j} \otimes \mathbb{1}_{V}\right)=\mathscr{D}_{x-y} .
$$

This shows that the Bogomolny data is simply shifted by $y$, which agrees with the action. For the reverse notice that the inverse of an equivariant map is automatically equivariant.

Proposition 7.39. The Nahm transform is equivariant with respect to the $\mathbf{S O}(3)$-actions

Proof. Again looking at the operator

$$
\mathscr{D}_{x}=\mathbb{1}_{\mathbb{C}^{2}} \otimes \nabla_{\mathrm{t}}+\left(\sum_{j=1}^{3} \sigma_{j} \otimes \mathrm{T}_{j}-i x_{j} \sigma_{j} \otimes \mathbb{1}_{V}\right),
$$

it now is convenient to denote $\vec{T}=i T_{1}+j T_{2}+k T_{3}$ and $\vec{x}=i x_{1}+j x_{2}+k x_{3}$, so that

$$
\mathscr{D}_{x}=\mathbb{1}_{\mathbb{C}^{2}} \otimes \nabla_{t}-\vec{T}+\vec{x} \otimes i \mathbb{1}_{V}
$$

We notice that if we act by $q \in \mathbf{S p}(1)$ on $T_{j}$ and use these to define the operator (without the regauging that is necessary only for the boundary conditions), we see that

$$
\mathscr{D}_{x}^{q}=\mathbb{1}_{\mathrm{C}} \otimes \nabla_{\mathrm{t}}-\mathrm{q} \overrightarrow{\mathrm{T}} \overline{\mathrm{q}}+\overrightarrow{\mathrm{x}} \otimes i \mathbb{1}_{V}=\mathrm{q} \mathscr{D}_{\overline{\mathrm{q}} \times \mathrm{q}} \overline{\mathrm{q}} .
$$

For a matrix $B \in \pi(q) \in \mathbf{S O}(3)$ this reads

$$
\mathscr{D}_{\mathrm{x}}=\mathrm{B} \mathscr{D}_{\mathrm{B}^{-1} \mathrm{x}} \text {. }
$$

Now the argument is the same as in Proposition 7.37 (compare to equation 7.171)).

Proposition 7.40. The extended Nahm Transform maps gauge equivalence classes to gauge equivalence classes..$^{2}$

Proof. This is true in much greater generality, see e.g. [Jaro4, Lemma 1]

\footnotetext{
${ }^{2}$ Technically speaking, there is a canonical isomorphism between the transformed bundles of two gauge equivalent connections induced by the gauge transformation of the original connections - and the connections agree under this isomorphism. Since all bundles are trivializable, these isomorphisms are indistinguishable from gauge transformation of the trivial bundle. See also the details in |Jaro4|.
} 


\subsection{Summary}

Let us summarize the results of this chapter. We noticed that we can extend the Nahm transform to an open neighborhood $\mathrm{U} \subset \mathrm{F}_{\mathrm{N}}$ of $\mu_{\mathrm{N}}^{-1}(0) \subset \mathrm{F}_{\mathrm{N}}$, and similarly to an open neighborhood of $\mu_{\mathrm{B}}{ }^{-1}(0) \subset \mathrm{F}_{\mathrm{B}}$. This is true because the conditions in Corollary $7 \cdot 30$ and Corollary 7.32 are open and satisfied on $\mu^{-1}(0)$. We have also shown that the extended Nahm transform is $\mathbf{S O}(3) \ltimes \mathbb{R}^{3}$-equivariant, commutes with homotheties and maps gauge equivalence classes to gauge equivalence classes.

An interesting question is if it can be extended to a even larger class of data, than what Corollary 7.30 and Corollary 7.32 suggest. This seems possible for two reasons. First, the derived properties in these corollaries are actually stronger than needed for the Nahm transform, and secondly we only used the property to have a handy expression of the Greens operator. It seems unlikely that it can be extended to all of $F_{B} / F_{N}$ though, as that would change the topology of the bundles involved in the transformation (compare to Remark 8.72). 


\section{Chapter 8}

\section{Identification of Gauge Theories}

In this chapter we will use the collected knowledge to formulate a relation between certain gauge theories. We will start by describing a construction that allows to identify gauge theories of dimensions less than four with gauges theories between dimensional four and eight. We shall then apply this construction to the examples of gauge theories we have discussed; relating solutions of the Nahm equations to solutions of the Haydys-Witten equations and solutions of the Bogomolny equations to $\mathbf{G}_{\mathbf{2}}$-Monopoles. Finally we will use the results of Chapter 7 to lift the relation of the Nahm equations to the Bogomolny equations to a relation of the Haydys-Witten equations to the $\mathbf{G}_{\mathbf{2}^{-}}$ Monopole equations.

\subsection{Prelude}

Lemma 8.1. Let $\mathrm{M}$ and $\mathrm{N}$ be two manifolds and $\mathrm{V}$ a finite dimensional vector space. Then $\mathfrak{C}^{\infty}(\mathrm{N}, \mathrm{V})$ has a natural vector space structure. Using this structure, the exponential law holds, i.e.

$$
\mathfrak{C}^{\infty}\left(M, \mathfrak{C}^{\infty}(\mathrm{N}, \mathrm{V})\right) \cong \mathfrak{C}^{\infty}(\mathrm{M} \times \mathrm{N}, \mathrm{V}) .
$$

Proof. See [KM97, Lemma 27.17].

Lemma 8.2. Let $\mathrm{E} \rightarrow \mathrm{M}$ be a smooth vector bundle on a manifold $\mathrm{M}$, and let

$$
s: \mathbb{R}^{k} \rightarrow \Gamma(E),
$$

be a family of sections. Then $\mathrm{s}$ is smooth if and only if $\hat{\mathrm{s}}: \mathbb{R}^{\mathrm{k}} \times \mathrm{M} \rightarrow \mathrm{E}$ is smooth with $\hat{s}(x, m)=s(x)(m)$.

Proof. This is a slight generalization of [KM97, Lemma 30.8] that follows from [KM97. Corollary 4.16].

Corollary 8.3. Let $\mathrm{E} \rightarrow \mathrm{M}$ be a vector bundle and $\mathrm{pr}_{M}^{*} \mathrm{E} \rightarrow \mathbb{R}^{\mathrm{k}} \times \mathrm{M}$ its pull back. Then

$$
\mathfrak{C}^{\infty}\left(\mathbb{R}^{k}, \Gamma(M, E)\right) \cong \Gamma\left(\mathbb{R}^{k} \times M, p^{*} E\right)
$$


Proof. All the hard analytic work is already done, we need to just check the algebra. A section in the pullback of $E$ to $\mathbb{R}^{k} \times M$ is given by a smooth map $\mathbb{R}^{k} \times M \rightarrow E$ that makes a commutative diagram with $\pi: E \rightarrow M$ and $\mathrm{pr}_{M}: \mathbb{R}^{k} \times M \rightarrow M$. But if $s \in \mathfrak{C}^{\infty}\left(\mathbb{R}^{k}, \Gamma(M, E)\right)$, then $\pi \circ \hat{s}(x, p)=\pi(s(x)(p))=p=\operatorname{pr}_{M}(x, p)$ for every $(x, p) \in$ $\mathbb{R}^{k} \times M$ and similarly the other way around.

Notation 8.4. We will call maps that intertwine G-actions G-equivariant, no matter if it is between left actions, right actions or mixed actions.

Proposition 8.5. Let $\mathrm{Q} \rightarrow \mathrm{X}$ be a principal $\mathrm{H}$ bundle and $\rho: \mathrm{H} \rightarrow \mathrm{Gl}(\mathrm{V})$ a representation of $\mathrm{H}$. Let further $\mathrm{P}$ be a reduction of $\mathrm{Q}$ to a principal $\mathrm{G}-$ bundle, with respect to $\lambda: \mathrm{G} \rightarrow \mathrm{H}$ and ı: $\mathrm{P} \rightarrow \mathrm{Q}$.

Then the vector bundles

$$
\mathrm{E}=\mathrm{Q} \times_{\mathrm{H}} \mathrm{V} \text { and } \mathrm{F}=\mathrm{P} \times_{\mathrm{G}} \mathrm{V},
$$

are canonically isomorphic, where the second bundle uses the induced representation $\mathrm{G} \rightarrow$ Gl(V) given by $\rho \circ \lambda$.

In particular the forms with values in these bundles can be canonically identified.

$$
\Omega^{\mathrm{k}}(\mathrm{X}, \mathrm{E}) \cong \Omega^{\mathrm{k}}(\mathrm{X}, \mathrm{F}), \quad \forall \mathrm{k} \in \mathbb{N}_{0} .
$$

Proof. Define the vector bundle map

$$
\mathrm{F} \rightarrow \mathrm{E}, \quad[\mathrm{p}, v] \mapsto[\mathrm{l}(\mathrm{p}), v]
$$

and notice that it is fiberwise an isomorphism, hence an isomorphism of vector bundles. The other claims follow immediately.

Remark 8.6. While the last proposition was easy to prove, it is not very descriptive how the identification works. The key to the construction are equivariant extensions. The reverse map $E \rightarrow F$ is constructed by picking a representative of $[q, v]$, such that $q \in \mathfrak{l}(P)$. Let us discuss the forms for the example with $k=0$.

Then sections of the bundle associated to $E$ are given by maps

$$
\mathrm{u}: \mathrm{Q} \rightarrow \mathrm{V}, \quad \mathrm{u} \text { is H-equivariant. }
$$

Similarly sections of $\mathrm{F}$ are G-equivariant maps $\mathrm{P} \rightarrow \mathrm{V}$.

If $\mathrm{u}: \mathrm{Q} \rightarrow \mathrm{V}$ is H-equivariant, then $\mathrm{u}^{\prime}: \mathrm{P} \rightarrow \mathrm{Q} \rightarrow \mathrm{V}$ is G-equivariant. If $v: \mathrm{P} \rightarrow \mathrm{V}$ is G-equivariant, we may extend $v$ H-equivariantly to $Q$. For $q \in Q$, pick $p \in P$ and $h \in H$ with $p h=q$ and define $v^{\prime}(q)=h^{-1} v(p)$.

- $v^{\prime}$ is well defined. If $q=p_{1} h_{1}=p_{2} h_{2}$, then $p_{1}=p_{2} h_{2} h_{1}^{-1}$ and $h_{2} h_{1}^{-1} \in G$, hence

$$
v_{1}^{\prime}(\mathrm{q})=\mathrm{h}_{1} v\left(\mathrm{p}_{1}\right)=\mathrm{h}_{1} v\left(\mathrm{p}_{2} \mathrm{~h}_{2} \mathrm{~h}_{1}^{-1}\right)=\mathrm{h}_{1}^{-1} \mathrm{~h}_{1} \mathrm{~h}_{2}^{-1} v\left(\mathrm{p}_{2}\right)=v_{2}^{\prime}(\mathrm{q}) .
$$


- $v^{\prime}$ is H-equivariant. Indeed, if $q \in Q, h, h^{\prime} \in H$ and $p \in P$ with $q=p h$ then

$$
v^{\prime}\left(\mathrm{qh}^{\prime}\right)=v^{\prime}\left(\mathrm{phh}^{\prime}\right)=\mathrm{h}^{-1} \mathrm{~h}^{-1} v^{\prime}(\mathrm{p})=\mathrm{h}^{-1} v^{\prime}(\mathrm{q}) .
$$

Lemma 8.7. Let $(\mathrm{M}, \mathrm{g})$ be an $\mathrm{n}$-dimensional Riemannian manifold and $\varepsilon \in \mathbb{R}^{>0}$. Then $\mathrm{g}$ and $\varepsilon^{2} \mathrm{~g}$ are two metrics on $\mathrm{M}$, and the induced Hodge stars are related by

$$
\left.\star_{g}\right|_{\Lambda^{k}}=\left.\varepsilon^{n-2 k} \star_{\varepsilon^{2} g}\right|_{\Lambda^{k}}
$$

Proof. Use the formula

$$
\omega \wedge \star \eta=\langle\omega, \eta\rangle \text { vol } \quad \omega, \eta \in \Omega^{k}(M),
$$

which uniquely determines the Hodge Star operator. Also note that

$$
\operatorname{vol}_{g}=\varepsilon^{n} \operatorname{vol}_{\varepsilon^{2} g}
$$

and restricted to $k$-forms on $M$,

$$
\langle-,-\rangle_{\varepsilon^{2} g}=\varepsilon^{2 k}\langle-,-\rangle_{g} .
$$

Lemma 8.8. Let $\mathrm{X}, \mathrm{Y}$ be two manifolds and $\mathrm{P} \rightarrow \mathrm{X}$ and $\mathrm{Q} \rightarrow \mathrm{Y}$ two principal $\mathrm{G}-$ bundles. Then there is a bijection between $\mathrm{G}$-equivariant maps $\mathrm{u}: \mathrm{P} \rightarrow \mathrm{Q}$ and maps $\check{\mathrm{u}}: \mathrm{X} \rightarrow \mathrm{Y}$ such that $\check{u}^{*} \mathrm{Q} \cong \mathrm{P}$.

Proof. First a G-equivariant map $\mathrm{P} \rightarrow \mathrm{Q}$ is equivalent to the following commuting square.

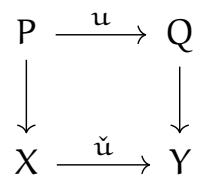

By general categorial considerations, such a square is equivalent to a morphism $\mathrm{P} \rightarrow \check{u}^{*} \mathrm{Q}$ of G-principal bundles over X. Finally every morphism of principal bundles is an isomorphism.

Remark 8.9. The last Lemma in particular implies that if we have a G-equivariant map $P \rightarrow Q$, then $P$ is the pull back of $Q$ by a map $\check{u}$. Now if $\check{u}$ is homotopic to $\check{v}$, then the pulled back bundles are isomorphic, the inverse is however only true if $\mathrm{Q}$ is contractible. So we can have different homotopy classes of maps $\check{u}: X \rightarrow Y$.

Notation 8.10. Just like chapter 5 we will use the notation where an indexed Lie Algebra means vanishing on the corresponding fundamental vector fields. E.g. $\omega \in \Omega(M)_{\mathfrak{g}}$ (with $G \frown M$ ) satisfies $\iota_{\mathfrak{g}} \omega=0$. 


\subsection{General Construction}

\subsubsection{Reduction of Gauge Theories on Vector Bundles}

Let $\mathrm{E} \rightarrow \mathrm{X}$ be a vector bundle and $\pi_{\mathrm{P}}: \mathrm{P} \rightarrow \mathrm{E}$ a principal G-bundle on $\mathrm{E}$. Denote by $\mathrm{Q}$ a principal $\mathrm{H}$-bundle to which $\mathrm{E}$ is associated via a representation $\rho: \mathrm{H} \rightarrow \operatorname{End}(\mathrm{V})$.

Since $E$ deformation retracts to $X, P$ comes from a bundle on $X, P \cong \pi_{E}^{*} P_{X}$, where $P_{X} \rightarrow X$ is a principal G-bundle.

On the other hand there is a one-to-one correspondence between equivariant bundles on the total space $Q \times V$ and regular bundles on the base space so that $P \rightarrow E$ corresponds to a H-equivariant bundle $\hat{\mathrm{P}} \rightarrow \mathrm{Q} \times \mathrm{V}$.

Lemma 8.11. The bundle $\hat{\mathrm{P}}$ comes from a $\mathrm{H}$-equivariant bundle on $\mathrm{Q}$.

Proof. Notice that we have the commutative box, where all principal bundles on top and are pull backs of $P_{X}$.

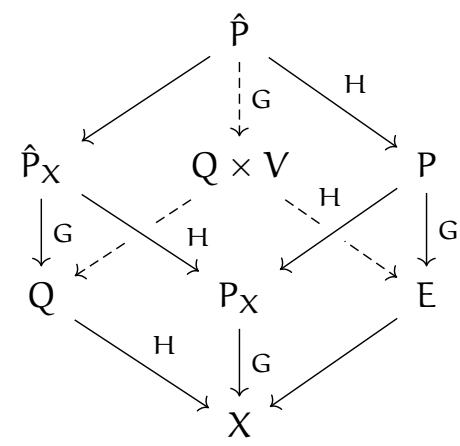

Corollary 8.12. As H-equivariant principal G-bundles $\hat{\mathrm{P}} \cong \hat{\mathrm{P}}_{\mathrm{X}} \times \mathrm{V}$.

Proof. By definition we have

$$
\hat{\mathrm{P}}=\pi_{\mathrm{Q}}^{*} \hat{\mathrm{P}}_{X}=\left\{(\mathrm{p},(\mathrm{q}, v)) \mid \pi_{\hat{\mathrm{P}}_{X}}(\mathrm{p})=\pi_{\mathrm{Q}}(\mathrm{q}, v)=\mathrm{q}\right\},
$$

and we have maps

$$
\hat{\mathrm{P}} \rightarrow \hat{\mathrm{P}}_{X} \times \mathrm{V}, \quad(\mathrm{p},(\mathrm{q}, v)) \mapsto(\mathrm{p}, v),
$$

and

$$
\hat{\mathrm{P}}_{X} \times \mathrm{V} \rightarrow \hat{\mathrm{P}}, \quad(\mathrm{p}, v) \mapsto\left(\mathrm{p},\left(\pi_{\hat{\mathrm{P}}_{X}}(\mathrm{p}), v\right)\right) .
$$

These are obviously inverse diffeomorphisms and if we equip $\hat{P}_{X} \times V$ with the bundle structure induced by

$$
\hat{\mathrm{P}}_{\mathrm{X}} \times \mathrm{V} \rightarrow \mathrm{Q} \times \mathrm{V}, \quad(\mathrm{p}, v) \mapsto \pi_{\hat{\mathrm{P}}_{X}}(\mathrm{p}),
$$


and

$$
(p, v) \cdot g=(p . g, v) \quad \forall(p, v) \in \hat{P}_{X} \times V, \forall g \in G,
$$

these maps become isomorphisms of principal G-bundles. Finally we see that the identifying maps are H-equivariant for the action

$$
(p, v) \cdot h=\left(p \cdot h, h^{-1} \cdot v\right),
$$

because $\pi_{\hat{\mathrm{P}}_{\mathrm{X}}}$ and $\pi_{\mathrm{Q}}$ is H-equivariant.

Proposition 8.13. In the notation above,

$$
\Gamma(\mathrm{E}, \operatorname{Ad}(\mathrm{P})) \cong \mathfrak{C}^{\infty}\left(\mathrm{V}, \Gamma\left(\mathrm{Q}, \operatorname{Ad}\left(\hat{\mathrm{P}}_{\mathrm{X}}\right)\right)\right)^{\mathrm{H}} .
$$

Proof.

$$
\begin{aligned}
& \Gamma(E, \operatorname{Ad}(P)) \cong \mathfrak{C}^{\infty}(P, \mathfrak{g})^{\mathrm{G}} \cong \mathfrak{C}^{\infty}(\hat{\mathrm{P}}, \mathfrak{g})^{\mathrm{G} \times \mathrm{H}} \\
& \cong \mathfrak{C}^{\infty}\left(\hat{\mathrm{P}}_{X} \times \mathrm{V}, \mathfrak{g}\right)^{\mathrm{G} \times \mathrm{H}} \cong \mathfrak{C}^{\infty}\left(\mathrm{V}, \mathfrak{C}^{\infty}\left(\hat{\mathrm{P}}_{X, \mathfrak{g}}\right)^{\mathrm{G}}\right)^{\mathrm{H}} .
\end{aligned}
$$

In the last step, the H-invariancy becomes an H-equivariancy in the following sense. For every $s \in \mathfrak{C}^{\infty}\left(\mathrm{V}, \Gamma\left(\mathrm{Q}, \operatorname{Ad}\left(\hat{\mathrm{P}}_{\mathrm{X}}\right)\right)\right)^{\mathrm{H}}$ and $v \in \mathrm{V}, \mathrm{h} \in \mathrm{H}$ we have

$$
s(h v)=s(v) \circ R_{h^{-1}} .
$$

Corollary 8.14. If $\mathrm{E}$ is the trivial vector bundle, then

$$
\Gamma(\mathrm{E}, \operatorname{Ad}(\mathrm{P})) \cong \mathfrak{C}^{\infty}(\mathrm{V}, \Gamma(\mathrm{X}, \operatorname{Ad}(\mathrm{Px})))
$$

Proof. This follows immediately from $H=1$ and $Q=X$, so that also $P_{X}=\hat{P}_{X}$.

Proposition 8.15. In the same setting of the last proposition, let W be some G-space (Grepresentation, or an arbitrary space with $\mathrm{G}$-action and $\mathrm{k}=0$ in the following). Then

$$
\Omega^{k}(P, W)^{G} \cong\left[\bigoplus_{i=0}^{k} \mathfrak{C}^{\infty}\left(V, \Lambda^{k-i} V^{\vee} \otimes \Omega^{i}\left(\hat{P}_{X}, W\right)^{G}\right)^{H}\right]_{\mathfrak{h}}
$$

Proof. We calculate

$$
\Omega^{k}(P, W)^{G} \cong \Omega^{k}(\hat{P}, W)_{\mathfrak{h}}^{G \times H} \cong \Omega^{k}\left(\hat{P}_{X} \times V, W\right)_{\mathfrak{h}}^{G \times H}
$$


We continute the calculation

$$
\begin{aligned}
\Omega^{k}(P, W)^{G} & \cong \Gamma\left(\hat{P}_{X} \times V, \Lambda^{k}\left(T^{\vee} \hat{P}_{X} \oplus T^{\vee} V\right) \otimes W\right)_{\mathfrak{h}}^{G \times H} \\
& \cong\left[\bigoplus_{i=0}^{k} \mathfrak{C}^{\infty}\left(V, \Gamma\left(\hat{P}^{X}, \Lambda^{i} T^{\vee} \hat{P}_{X} \otimes \Lambda^{k-i} V^{\vee}\right)^{G}\right)^{H}\right]_{\mathfrak{h}} \\
& \cong\left[\bigoplus_{i=0}^{k} \mathfrak{C}^{\infty}\left(V, \Lambda^{k-i} V^{\vee} \otimes \Omega^{i}\left(\hat{P}_{X}, W\right)^{G}\right)^{H}\right]_{\mathfrak{h}} .
\end{aligned}
$$

The action of $\mathrm{H}$ on $\mathrm{V}$ is identical to the induced action of $\mathrm{H}$ on $\mathrm{T}_{v} \mathrm{~V}$ under the (canonical) identification $T_{\nu} V \cong V$.

Corollary 8.16. $\quad$ - If $\mathrm{E}$ is trivial, then the last proposition becomes

$$
\Omega^{k}(P, W)^{G} \cong \bigoplus_{i=0}^{k} \mathfrak{C}^{\infty}\left(V, \Lambda^{k-i} V^{\vee} \otimes \Omega^{i}\left(\hat{P}_{X}, W\right)^{G}\right)
$$

- If $\mathrm{\omega}$ is a form on $\mathrm{E}$ with values in the associated bundle $\mathrm{F}=\mathrm{P} \times{ }_{\mathrm{G}} \mathrm{W}$ (for a vector $\mathrm{G}$-space $\mathrm{W})$, then an inspection shows that the $\mathfrak{g}$-horizontality commutes with the construction. We get

$$
\Omega^{k}(E, F) \cong\left[\bigoplus_{i=0}^{k} \mathfrak{C}^{\infty}\left(V, \Lambda^{k-i} V^{\vee} \otimes \Omega^{i}(Q, \hat{F})\right)^{H}\right]_{\mathfrak{h}},
$$

where $\hat{\mathrm{F}}$ is the bundle associated to $\hat{\mathrm{P}}_{\mathrm{X}}$ and $\mathrm{W}$.

- If $\omega$ is a form on $\mathrm{E}$ horizontal w.r.t. $\mathrm{E} \rightarrow \mathrm{X}$, then $\omega$ lives in the last summand of the last proposition. We get

$$
\Omega^{\mathrm{k}}(\mathrm{E}, \mathrm{F})_{\mathrm{hor}} \cong \mathfrak{C}^{\infty}\left(\mathrm{V}, \Omega^{\mathrm{k}}(\mathrm{Q}, \hat{\mathrm{F}})_{\mathfrak{h}}\right)^{\mathrm{H}}
$$

Remark 8.17. Let us look at the last proposition for the special case of $\Omega^{1}(\mathrm{P}, \mathfrak{g})^{\mathrm{G}}$ for a moment. The proposition tells us that

$$
\Omega^{1}(\mathrm{P}, \mathfrak{g})^{\mathrm{G}} \cong\left[\mathfrak{C}^{\infty}\left(\mathrm{V}, \mathrm{V}^{\vee} \otimes \mathfrak{C}^{\infty}\left(\hat{\mathrm{P}}_{\mathrm{X}, \mathfrak{g})}\right)^{\mathrm{G}}\right)^{\mathrm{H}} \oplus \mathfrak{C}^{\infty}\left(\mathrm{V}, \Omega^{1}\left(\hat{\mathrm{P}}_{\mathrm{X}}, \mathfrak{g}\right)^{\mathrm{G}}\right)^{\mathrm{H}}\right]_{\mathfrak{h}}
$$

To realize this splitting, we start with a G-equivariant one form $A \in \Omega^{1}(P, \mathfrak{g})^{G}$ and pull it back to $\hat{P}$. Since $\hat{P} \cong \hat{P}_{X} \times V$, we can decompose the pull back by restricting to $T \hat{P}_{X}$ and TV. Since $V$ acts on $\hat{P}$ by the above decomposition, the second restriction may be identified with $\imath_{V} \pi_{\hat{\mathrm{P}}}^{*} A$, where $\pi_{\hat{\mathrm{P}}}: \hat{\mathrm{P}} \rightarrow \mathrm{P}$. This corresponds to the restriction of $A$ to the vertical subbundle.

Finally we use 8.1 to identify the two components. We may write symbolically

$$
A \sim(c, a)=\left(\operatorname{lv} \pi_{\hat{p}}^{*} A,\left.\pi_{\hat{p}}^{*} A\right|_{T \hat{P}_{x}}\right) .
$$


Proposition 8.18. In the notation of the Remark above, if $\mathrm{A} \in \mathrm{C}(\mathrm{P})$ is a connection on $\mathrm{P}$, then a is a family of connections on $\hat{P}_{X}$, i.e.

$$
a \in \mathfrak{C}^{\infty}\left(\mathrm{V}, \mathcal{C}\left(\hat{\mathrm{P}}_{X}\right)\right)^{\mathrm{H}} .
$$

Proof. We have already established that a takes values in the G-equivariant 1-forms on $\hat{P}_{X}$ with values in $\mathfrak{g}$. What is left to show is that

$$
\mathfrak{l}_{\mathfrak{g}} \mathrm{a}(v)=\mathrm{id}_{\mathfrak{g}} \quad \forall v \in \mathrm{V} .
$$

For this, note that if $X$ and $Y$ are two G-spaces, $f: X \rightarrow Y$ is a G-map, $W$ is some G-representation and $\omega \in \Omega^{1}(Y, W)^{G}$, then

$$
\left(\mathfrak{l}_{\mathfrak{g}}\left(f^{*} \omega\right)_{\chi}\right)(\xi)=\left(f^{*} \omega\right)_{\chi}\left(K_{\chi}^{\xi}\right)=\omega_{f(x)}\left(D f_{x} K_{\chi}^{\xi}\right)=\omega_{f(x)}\left(K_{f(x)}^{\xi}\right)=\left(f^{*}\left(\mathfrak{l}_{\mathfrak{g}} \omega\right)\right)_{\chi}(\xi),
$$

i.e. as maps $X \rightarrow \operatorname{Hom}(\mathfrak{g}, W)^{G}, \mathfrak{l}_{\mathfrak{g}}\left(f^{*} \omega\right)$ agrees with $f^{*}\left(\mathfrak{t}_{\mathfrak{g}} \omega\right)$. Now notice that $\hat{P} \rightarrow P$ (as a pull back map) is G-equivariant, as is the identification $\hat{P} \cong \hat{P}_{X} \times V$. Finally, the action of $G$ on $\hat{P}_{X} \times V$ is only on the first component, so the restriction to $T \hat{P}_{X}$ is G-equivariant. This shows, that

$$
\mathfrak{l}_{\mathfrak{g}} \mathrm{a}(v)=\mathfrak{\iota}_{\mathfrak{g}} A=\mathrm{id}_{\mathfrak{g}}
$$

at every $\hat{p} \in \hat{P}_{X}$.

\subsubsection{Compensation through bigger Structure Groups}

The last section allowed us to identify the connection on $\mathrm{P}$ with smooth maps into various section spaces of bundles on $\mathrm{Q}$. We can get rid of the smooth maps part if we enlarge the structure groups of the involved bundles.

Definition 8.19. Note that $\hat{P}_{X} \cong \mathrm{Q} \times_{X} \mathrm{P}_{X}$ as principal $\mathrm{G}$ bundles. Now denote by $\mathcal{G}=\mathfrak{C}^{\infty}(\mathrm{V}, \mathrm{G})$. Then we can interpret $\mathrm{G}$ as the subgroup of constant maps in $\mathcal{G}$, and define $\mathrm{P}^{\prime}$ to be the fiber extension of $\mathrm{P}_{\mathrm{X}}, \mathrm{P}^{\prime}=\mathrm{P}_{\mathrm{X}} \times_{G} \mathcal{G}$. Similarly we extend $\hat{\mathrm{P}}_{\mathrm{X}}$ to $\hat{\mathrm{P}}^{\prime}$ by the map

$$
\operatorname{id}_{\mathrm{H}} \times \text { const }_{\mathrm{G}}: \quad \mathrm{H} \times \mathrm{G} \rightarrow \mathrm{H} \ltimes \mathcal{G} .
$$

It is easy to see that

$$
\hat{\mathrm{P}}^{\prime} \cong \mathrm{Q} \times \mathrm{P}^{\prime}
$$

Remark 8.20. Note that $\mathrm{H}$ has a nontrivial action on $\mathcal{G}$ by composition. This implies that $\mathrm{Q} \times{ }_{\mathrm{X}} \mathrm{P}^{\prime}$ has the structure group $\mathrm{H} \ltimes \mathcal{G}$. Of course the subgroup $\mathrm{G} \subset \mathcal{G}$ is a pointwise stabilizer of the $\mathrm{H}$ action.

Proposition 8.21. For any G-space W, we have

$$
\mathfrak{C}^{\infty}\left(\mathrm{V}, \mathfrak{C}^{\infty}\left(\hat{\mathrm{P}}_{X}, \mathrm{~W}\right)^{\mathrm{G}}\right)^{\mathrm{H}} \cong \mathfrak{C}^{\infty}\left(\hat{\mathrm{P}}^{\prime}, \mathfrak{C}^{\infty}(\mathrm{V}, \mathrm{W})\right)^{\mathfrak{} \times \mathrm{H}}
$$


Proof. Starting at the right side, we can apply Proposition 8.5 to see that

$$
\mathfrak{C}^{\infty}\left(\hat{\mathrm{P}}^{\prime}, \mathfrak{C}^{\infty}(\mathrm{V}, \mathrm{W})\right)^{\mathcal{G} \rtimes \mathrm{H}}=\mathfrak{C}^{\infty}\left(\hat{\mathrm{P}}_{\mathrm{X}}, \mathfrak{C}^{\infty}(\mathrm{V}, \mathrm{W})\right)^{\mathrm{G} \times \mathrm{H}},
$$

where the action of $G \subset \mathfrak{C}^{\infty}(\mathrm{V}, \mathrm{G})$ is via the inclusion.

Using Lemma 8.1 twice, we see that the right hand side of the last equation is given by $\mathfrak{C}^{\infty}\left(\mathrm{V}, \mathfrak{C}^{\infty}\left(\hat{\mathrm{P}}_{\mathrm{X}}, \mathfrak{g}\right)^{\mathrm{G}}\right)^{\mathrm{H}}$, showing the equality.

Proposition 8.22. We may generalize the last proposition to the statement

$$
\mathfrak{C}^{\infty}\left(V, \Lambda^{k-i} V^{\vee} \otimes \Omega^{i}\left(\hat{P}_{X}, W\right)^{G}\right)^{H} \cong \Omega^{i}\left(\hat{P}^{\prime}, \Lambda^{k-i} V^{\vee} \otimes \mathfrak{C}^{\infty}(V, W)\right)^{9 \rtimes H}
$$

Proof. The proof is similar to the last proposition, but we use Lemma 8.2 instead of Lemma 8.1.

\section{Corollary 8.23.}

$$
\mathfrak{C}^{\infty}\left(\mathrm{V}, \mathfrak{C}^{\infty}\left(\hat{\mathrm{P}}_{X, \mathfrak{g})}\right)^{\mathrm{G}}\right)^{\mathrm{H}} \cong \mathfrak{C}^{\infty}\left(\hat{\mathrm{P}}^{\prime}, \operatorname{Lie}(\mathcal{G})\right)^{\mathfrak{G} \times \mathrm{H}} .
$$

Proof. Proposition 8.21 for $W=\mathfrak{g}$.

\section{Proposition 8.24.}

$$
\mathfrak{C}^{\infty}\left(\mathrm{V}, \mathrm{C}\left(\hat{\mathrm{P}}_{\mathrm{X}}\right)\right)^{\mathrm{H}} \cong \mathrm{C}\left(\hat{\mathrm{P}}^{\prime}\right)^{\mathrm{H}}
$$

Remark 8.25. It may be tempting to try to prove this with a small extension of Proposition 8.5 to forms that are not horizontal. Note however that this is impossible, since it is unclear what the forms values on fundamental vector fields of the bigger group (the vertical vector fields of the extension) are supposed to be.

However, in this case we can overcome this gap, by knowing what the values of the extended form on vertical vector fields has to be. What is left, is a choice of splitting into vertical and horizontal vector fields. That can be accomplished by either using the connections themselves or by choosing any fixed connection and showing invariancy under this choice. We follow the first path.

Proof. Let us begin with a smooth map $A \in \mathfrak{C}^{\infty}\left(\mathrm{V}, \mathrm{C}\left(\hat{\mathrm{P}}_{\mathrm{X}}\right)\right)^{\mathrm{H}}$. Using the fact that we can extend any connection from $\hat{P}_{X}$ to $\hat{P}^{\prime}$ (this extension is also H-equivariant), we have a map

$$
\mathcal{C}\left(\hat{\mathrm{P}}_{\mathrm{X}}\right) \rightarrow \mathcal{C}\left(\hat{\mathrm{P}}^{\prime}\right)
$$

inducing an element

$$
\hat{A} \in \mathfrak{C}^{\infty}\left(V, \mathcal{C}\left(\hat{P}^{\prime}\right)\right)^{\mathrm{H}} .
$$


Via Lemma 8.1. we identify this with an element in $\Omega^{1}\left(\hat{\mathrm{P}}^{\prime}, \mathfrak{C}^{\infty}(\mathrm{V} \times \mathrm{V}, \mathfrak{g})\right)^{\mathcal{G} \times \mathrm{H}}$ as follows. First note that $\operatorname{Lie}(\mathcal{G})=\mathfrak{C}^{\infty}(\mathrm{V}, \mathfrak{g})$ and

$$
\begin{aligned}
\mathfrak{C}^{\infty}\left(\mathrm{V}, \Omega^{1}\left(\hat{\mathrm{P}}^{\prime}, \mathrm{Lie}(\mathcal{G})\right)\right)^{\mathrm{H}} & \cong \mathfrak{C}^{\infty}\left(\mathrm{V}, \mathfrak{C}^{\infty}\left(\pi_{\hat{\mathrm{P}}^{\prime}}^{*} \mathrm{Fr}_{\mathrm{Gl}}\left(\mathrm{P}^{\prime}\right), \mathrm{U} \otimes \mathfrak{C}^{\infty}(\mathrm{V}, \mathfrak{g})\right)\right)^{\mathrm{Gl} \times(\mathcal{G} \rtimes \mathrm{H})} \\
& \left.\cong \mathfrak{C}^{\infty}\left(\pi_{\hat{\mathrm{P}}^{\prime}}^{*} \mathrm{Fr}_{\mathrm{Gl}}\left(\mathrm{P}^{\prime}\right), \mathrm{U} \otimes \mathfrak{C}^{\infty}(\mathrm{V} \times \mathrm{V}, \mathfrak{g})\right)\right)^{\mathrm{Gl} \times(\mathcal{G} \rtimes \mathrm{H})} \\
& \cong \Omega^{1}\left(\hat{\mathrm{P}}^{\prime}, \mathfrak{C}^{\infty}(\mathrm{V} \times \mathrm{V}, \mathfrak{g})\right)^{\mathfrak{G} \times \mathrm{H}}
\end{aligned}
$$

where $\mathrm{Gl}\lrcorner \mathrm{U}$ is the representation associated to $\pi_{\hat{\mathrm{P}}^{\prime}}^{*} T \mathrm{P}^{\prime}$ and $\pi_{\hat{\mathrm{P}}^{\prime}}^{*} \mathrm{Fr}_{\mathrm{Gl}}\left(\mathrm{P}^{\prime}\right)$ the G1-bundle of frames (compare to chapter 3 ). The diagonal map $\Delta: \mathrm{V} \rightarrow \mathrm{V} \times \mathrm{V}$ yields an element

$$
A^{\prime} \in \Omega^{1}\left(\hat{P}^{\prime}, \mathfrak{C}^{\infty}(\mathrm{V}, \mathfrak{g})\right)^{\mathcal{G} \rtimes \mathrm{H} .}
$$

Let us show that $A^{\prime} \in \mathcal{C}\left(\hat{P}^{\prime}\right)^{\mathrm{H}}$. We only need to show that $A$ has the correct values on fundamental vector fields of $\mathcal{G}$. Let $\xi \in \operatorname{Lie}(\mathcal{G})$. Then for all $v \in \mathrm{V}$,

$$
A^{\prime}(\xi)(v)=\hat{A}(v)\left(K^{\xi}\right)(v)=\xi(v),
$$

because $\hat{A}(v)$ is a connection on $\hat{\mathrm{P}}^{\prime}$, hence $\hat{\mathrm{A}}(v)\left(\mathrm{K}^{\xi}\right)=\xi$ for any $v$.

For the other way around, start with a connection $A^{\prime} \in \mathcal{C}\left(\hat{\mathrm{P}}^{\prime}\right)^{\mathrm{H}}$ and identify it with a map $\tilde{A} \in \mathfrak{C}^{\infty}\left(\mathrm{V}, \Omega^{1}\left(\hat{\mathrm{P}}^{\prime}, \mathfrak{g}\right)\right)^{\mathcal{G} \rtimes \mathrm{H}}$. Then using the reduction map $i: \hat{P}_{X} \rightarrow \hat{\mathrm{P}}^{\prime}$, which is $\mathrm{H}$ and equivariant with respect to $G \rightarrow \mathcal{G}, g \mapsto c_{g}$, we can pull back this to

$$
A \in \mathfrak{C}^{\infty}\left(\mathrm{V}, \Omega^{1}\left(\hat{\mathrm{P}}_{X}, \mathfrak{g}\right)\right)^{\mathrm{G} \times \mathrm{H}} .
$$

We claim that this takes values in the space of connections. Indeed, if $\xi \in \mathfrak{g}$, then

$$
A(v)\left(K_{G}^{\xi}\right)=\tilde{A}(v)\left(\operatorname{Di}\left(K_{G}^{\xi}\right)\right)=\tilde{A}(v)\left(K_{g}^{c \xi}\right)=c_{\xi}(v)=\xi,
$$

where we have used that the G-equivariant map $i$ maps fundamental vector fields to fundamental vectorfields, i.e.

$$
\operatorname{Di}\left(K_{G}^{\xi}\right)=K_{g}^{c_{\xi}}, \quad c_{\xi}=\operatorname{Dl}(\xi) .
$$

Finally let us remark how to see that these maps are inverse of each other. Recall that if we extend a connection $A(v)$ to $\tilde{A}(v)$, then the latter is characterized by

$$
A(v)=i^{*} \tilde{A}(v), \quad \forall v \in V .
$$

Hence if we start with $A$, map it to $A^{\prime}$ and then back to

$$
B(v)=i^{*} A^{\prime}(v)=\left(i^{*} \tilde{A}(v)\right)(v)=A(v) .
$$


Remark 8.26. Notice that we have used the inclusion $G \hookrightarrow \mathfrak{C}^{\infty}(\mathrm{V}, \mathrm{G})$ by constant maps here, but this can be generalized somewhat. The only problematic step is the reverse step of Proposition 8.24. Here we can patch the theorem by requiring the inclusion $\mathrm{G} \rightarrow \mathfrak{C}^{\infty}(\mathrm{V}, \mathrm{G})$ be as constant maps on a large open subset $\mathrm{U} \subset \mathrm{V}$; then the resulting one forms in $\mathfrak{C}^{\infty}\left(\mathrm{V}, \Omega^{1}\left(\hat{\mathrm{P}}_{\mathrm{X}}\right)^{\mathrm{G}}\right)^{\mathrm{H}}$ will only be a connection for $v \in \mathrm{U}$.

We are interested in another class of inclusions here. Take some function $f \in \mathfrak{C}^{\infty}(\mathrm{V})$ and integrate the Lie Algebra map

$$
\mathfrak{g} \ni \xi \mapsto \xi \mathfrak{f} \in \mathfrak{C}^{\infty}(\mathrm{V}, \mathfrak{g})=\operatorname{Lie}(\mathcal{G}),
$$

to a map of Lie groups (if $\pi_{1}(G) \neq 0$, this is not unique). This gives a homomorphism $G \rightarrow \mathcal{G}$. If we assume that $f \neq 0$ on all of $V$ and that $f$ is $H$-invariant (so that $G \subset \mathcal{G}$ is a pointwise H-stable) then the induced form in Proposition 8.24 will not be a connection, but $\frac{1}{f}$ A will be (compare to equation 8.55 )

$$
\mathrm{A}(v)\left(\mathrm{K}_{\mathrm{G}}^{\xi}\right)=\tilde{A}(v)\left(\mathrm{Di}\left(\mathrm{K}_{\mathrm{G}}^{\xi}\right)\right)=\tilde{A}(v)\left(\mathrm{K}_{\mathcal{G}}^{\mathrm{f \xi}}\right)=(\mathrm{f} \xi)(v)=\mathrm{f}(v) \xi .
$$

This way the theorem will remain true with this modification.

Remark 8.27. Note that we can use Proposition 8.15 and 8.21 to transform the gauge group $\mathcal{G}(\mathrm{P})=\mathfrak{C}^{\infty}(\mathrm{P}, \mathrm{G})^{\mathrm{G}}$ to

$$
\mathfrak{C}^{\infty}\left(\hat{\mathrm{P}}^{\prime}, \mathcal{G}\right)^{\mathcal{G} \rtimes \mathrm{H}}
$$

which is the gauge group of $\hat{P}^{\prime}$ restricted to gauges that lift the identity gauge on $\mathrm{Q}$. We trace the action on the data to see how it acts on the other side in the following proposition.

Proposition 8.28. Let $\mathrm{A} \in \mathcal{C}(\mathrm{P})$ be a connection on $\mathrm{P}, \phi \in \Gamma(\operatorname{Ad}(\mathrm{P}))$ be a section of the adjoint bundle and $\mathrm{g} \in \mathcal{G}(\mathrm{P})$ a gauge. Let $(\mathrm{a}, \mathrm{c}) \in \mathcal{C}\left(\hat{\mathrm{P}}^{\prime}\right)^{\mathrm{H}} \times \mathfrak{C}^{\infty}\left(\hat{\mathrm{P}}^{\prime}, \mathrm{V}^{\vee} \otimes \operatorname{Lie}(\mathcal{G})\right)^{\mathcal{G} \times \mathrm{H}}$ and $\mathrm{b} \in \mathfrak{C}^{\infty}\left(\hat{\mathrm{P}}^{\prime}, \operatorname{Lie}(\mathcal{G})\right)^{\mathcal{G} \times \mathrm{H}}$ be the transformed data. Then the transformation of A.g and $\phi . \mathrm{g}$ is given by

$$
\left(\operatorname{Ad}_{\tilde{g}^{-1}} a+\tilde{g}^{*} \mu_{\mathcal{g}}, \operatorname{Ad}_{\tilde{g}^{-1}} R_{\tilde{g}}^{*} c\right)
$$

and

$$
\operatorname{Ad}_{\tilde{g}^{-1}} b,
$$

where $\tilde{\mathrm{g}}$ is the transformed gauge (see the last remark).

Proof. The proof consists mainly of going through the steps and seeing how the action of the gauge group is modified. The interesting step is for the connection $A \in \mathcal{C}(P)$, where $g \in \mathcal{G}(P)$ acts by

$$
\text { A.g }=\operatorname{Ad}_{g^{-1}} A+g^{*} \mu_{G} .
$$

When we split the pull back of $A$ to $\hat{P}$ into the components $(c, a)$ as in (8.35), we see that

$$
a . g=\operatorname{Ad}_{g^{-1}} a+g^{*} \mu_{G}, \quad \text { c.g }=\operatorname{Ad}_{g^{-1}} c
$$


since $\iota_{V} g^{*} \mu_{G}=0$ (on the identification with $\hat{P}_{X} \times V$ ). This remains true on $\hat{P}$, just the equations are true for every $v \in V$. In the compensation step we enlarge the group of gauges, but using Proposition 8.5 (for the slight generalization from vector bundles to fiber bundles), we see that the gauge group of the extended bundle is isomorphic to the original gauge group.

\subsection{Examples}

We will now apply the theory to two examples, starting with the Haydys-Witten equations. The general idea to apply this construction is a 3-step procedure. The first step is to assume that the frame bundle of the underlying 5-dimensional manifold reduces algebraically to $\mathbf{S O}(4)$ and split the equations via the representation theory. The next step is to assume that the $\mathbf{S O}(4)$-structure comes from an underlying 4-dimensional manifold; i.e. we are on a vector bundle of a 4-manifold. This is used to describe the data of the equations as living on the underlying manifold. Finally we use section 8.2.2 to enlarge the gauge group and describe the equations as generalized Seiberg-Witten equations with infinite dimensional targets.

\subsubsection{Haydys-Witten Instantons}

Assume $M=\mathbb{R} \times X, X$ Riemannian, oriented 4-manifold, $P \stackrel{G}{\rightarrow} M$ a principal G-bundle, and $Y=\partial_{t}$ the canonical vector field along $\mathbb{R}$. Assume further that $(A, B) \in \mathcal{C}(P) \times$ $\Omega_{+}^{2}(\operatorname{Ad}(P))$ is a solution to the Haydys-Witten equations

$$
\begin{aligned}
\iota_{Y} F^{A}-\delta_{+}^{A} B=0 & \in \Omega^{1}(\operatorname{Ad}(P)), \\
F_{+}^{A}-\nabla_{Y}^{A} B-\sigma(B, B)=0 & \in \Omega_{+}^{2}(\operatorname{Ad}(P)) .
\end{aligned}
$$

The first step is not required for Haydys-Witten instantons, since they are already defined on a manifold with SO(4)-structure. For the second step, let $\mathrm{P}_{X} \rightarrow X$ denote the principal G-bundle which induces $P$ as in section 8.2.1.

Then using Proposition 8.15 and 8.18 , we can associate $A$ with a pair

$$
(a, c) \in \mathfrak{C}^{\infty}\left(\mathbb{R}, \mathcal{C}\left(P_{X}\right)\right) \times \mathfrak{C}^{\infty}\left(\mathbb{R}, \mathfrak{C}^{\infty}\left(P_{X}, \mathfrak{g}\right)^{G}\right)
$$

and similarly $\mathrm{B} \in \Omega_{+}^{2}(\mathrm{Ad}(\mathrm{P}))$ with an element

$$
\mathrm{b} \in \mathfrak{C}^{\infty}\left(\mathbb{R}, \Omega_{+}^{2}\left(\mathrm{X}, \operatorname{Ad}\left(\mathrm{P}_{X}\right)\right) .\right.
$$

Lemma 8.29. The Haydys-Witten equations in differential equations form (compare Remark 5.6) are given by

$$
\begin{array}{ll}
\dot{\mathrm{a}}=\left(\mathrm{d}_{+}^{\mathrm{a}}\right)^{*} \mathrm{~b}+\mathrm{d}_{\mathrm{a}} \mathrm{c}, & \in \mathfrak{C}^{\infty}\left(\mathbb{R}, \Omega^{1}\left(\mathrm{X}, \operatorname{Ad}\left(\mathrm{P}_{\mathrm{X}}\right)\right)\right) \\
\dot{\mathrm{b}}=\mathrm{F}_{+}^{\mathrm{a}}-\sigma(\mathrm{b}, \mathrm{b})-[\mathrm{c}, \mathrm{b}], & \in \mathfrak{C}^{\infty}\left(\mathbb{R}, \Omega_{+}^{2}\left(\operatorname{Ad}\left(\mathrm{P}_{\mathrm{X}}\right)\right)\right) .
\end{array}
$$




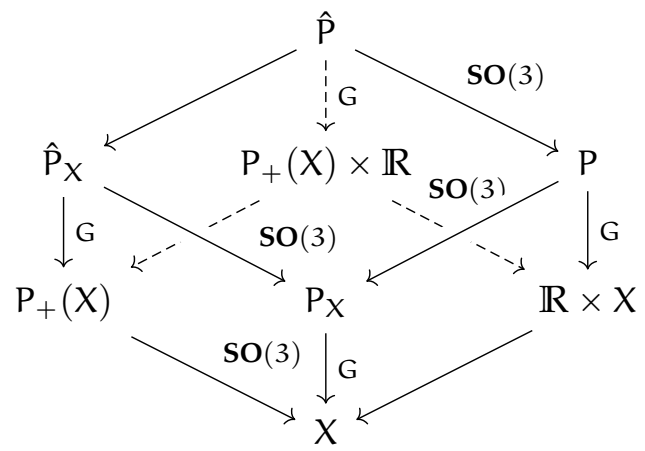

Figure 8.1: Involved principal bundles for Haydys-Witten Instantons

Proof. See [Hay15b].

In the last step we may compensate by the bigger structure group of $\mathrm{P}^{\prime}$. Note that we make use of the fact that the $\mathrm{H}$ action on $\mathcal{G}$ is trivial in this example, so that the structure group is simply given by $\mathcal{G} \times \mathrm{H}$. Using Proposition 8.21 and 8.24 we may identify a with an element in $\mathcal{C}\left(\mathrm{P}^{\prime}\right)$, b with an element in $\Omega_{+}^{2}(\operatorname{Ad}(\mathrm{P}))$ and $\mathrm{c}$ with an element in $\Gamma\left(\mathrm{X}, \operatorname{Ad}\left(\mathrm{P}^{\prime}\right)\right)$. The following relates the $5 \mathrm{~d}$-Haydys Witten equations on $M$ with solutions of the generalized Seiberg-Witten equations.

We worked on $M=\mathbb{R} \times X$ for simplicity so far, but in order to get the Nahm moduli space as a target space we have to modify this to $M=I \times X$. Of course the following theorem remains true for $\mathbb{R} \times X$ if we modify the space $W_{B}$ to be $\mathfrak{C}^{\infty}(\mathbb{R}, \mathfrak{g} \otimes \mathbb{H})$.

Theorem 8.30. A pair

$$
(A, B) \in \mathcal{C}(P) \times \Omega_{+}^{2}(\operatorname{Ad}(P))
$$

satisfies the Haydys-Witten equations

$$
\begin{aligned}
\iota_{Y} F^{A}-\delta_{+}^{A} B=0 & \in \Omega^{1}(\operatorname{Ad}(P)), \\
F_{+}^{A}-\nabla_{Y}^{A} B-\sigma(B, B)=0 & \in \Omega_{+}^{2}(\operatorname{Ad}(P)) .
\end{aligned}
$$

if and only if the associated triple (associated as explained in equations (8.68) and (8.69))

$$
\begin{aligned}
& a \in \mathcal{C}\left(\mathrm{P}^{\prime}\right) \\
& b \in \Omega_{+}^{2}\left(\operatorname{Ad}\left(\mathrm{P}^{\prime}\right)\right) \\
& c \in \Gamma\left(X, \operatorname{Ad}\left(\mathrm{P}^{\prime}\right)\right)
\end{aligned}
$$

satisfy the generalized Seiberg-Witten equations with target $\mathrm{W}_{\mathrm{N}}$, i.e.

$$
\mathscr{D}_{\mathrm{a}} \mathrm{u}=0, \quad \mu_{\mathrm{N}} \circ \mathrm{u}=\mathrm{F}_{+}^{\mathrm{a}},
$$


where $\mathrm{u}=\mathrm{c}+\mathrm{b} \in \mathfrak{C}^{\infty}\left(\mathrm{P}_{+} \times{ }_{\mathrm{X}} \mathrm{P}^{\prime}, \mathrm{W}_{\mathrm{N}}\right)^{\mathrm{SO}(3) \times \mathcal{G}}$ and

$$
\mu_{N} \circ u=\left(\begin{array}{l}
\dot{u}_{1}+\left[u_{0}, u_{1}\right]+\left[u_{2}, u_{3}\right] \\
\dot{u}_{2}+\left[u_{0}, u_{2}\right]+\left[u_{3}, u_{1}\right] \\
\dot{u}_{3}+\left[u_{0}, u_{3}\right]+\left[u_{1}, u_{2}\right]
\end{array}\right)
$$

and $\mathrm{P}_{+}=\mathrm{Q}$ (compare to diagram in Lemma 8.11) is the principal $\mathbf{S O}(3)$-bundle associated to $\Lambda_{+}^{2} \mathrm{X}$.

Proof. First we will show that $\mu \circ u=F_{+}^{a}$ if and only if the second equation of the Haydys-Witten equations in differential equation form holds. For that recall that our identification is

$$
\mathrm{u}_{0}=\mathrm{c}, \quad \mathrm{b}=\left(\mathrm{u}_{1}, \mathrm{u}_{2}, \mathrm{u}_{3}\right) .
$$

Using the identification $\Lambda_{+}^{2} \cong \operatorname{Im} \mathbb{H}$, we see that in some frame $f_{+}$of $P_{+}$(induced by $d x^{i}$ of $\left.\operatorname{Fr}_{S O(4)}(X)\right)$, i.e.

$$
f_{+}^{1}=d x^{12}+d x^{34} \quad f_{+}^{2}=d x^{13}-d x^{24} \quad f_{+}^{3}=d x^{14}+d x^{23},
$$

we have

$$
b=\sum_{i=1}^{3} u_{i} f_{+}^{i}
$$

and we calculate

$$
\sigma(b, b)=\left(\left[u_{2}, u_{3}\right],\left[u_{3}, u_{1}\right],\left[u_{1}, u_{2}\right]\right),
$$

and

$$
[c, b]=\left(\left[u_{0}, u_{1}\right],\left[u_{0}, u_{2}\right],\left[u_{0}, u_{3}\right]\right) .
$$

so that $\mu_{N} \circ u=\dot{b}+\sigma(b, b)+[c, b]$. This shows the equivalence of the second equations.

For the first equations, we will again use local coordinates. In such, we have

$$
a=\sum_{i=1}^{4} a_{i} d x^{i}, \quad b=\sum_{i=1}^{3} b_{i} f_{+}^{i} ;
$$

and so

$$
\begin{gathered}
\mathrm{d}_{\mathrm{a}} \mathrm{c}=\mathrm{dc}+[\mathrm{a}, \mathrm{c}]=\sum_{i=1}^{3}\left(\partial_{i} \mathrm{c}+\left[\mathrm{a}_{i}, \mathrm{c}\right]\right) \mathrm{d} x^{i}=\sum_{i=1}^{3} \nabla_{i}^{a} c d x^{i} \\
\dot{\mathrm{a}}=\sum_{i=1}^{4} \dot{\mathrm{a}}_{i} \mathrm{~d} x^{i}
\end{gathered}
$$

About $\left(d_{a}^{+}\right)^{*}$. Note that $d_{+}^{a}=\pi_{+} \circ d_{a}: \Omega^{1} \rightarrow \Omega_{+}^{2}$, hence

$$
\left(d_{a}^{+}\right)^{*}=\left(\pi_{+} \circ d_{a}\right)^{*}=d_{a}^{*} \circ \pi_{+}^{*}=d_{a}^{*} \circ \pi_{+}
$$


and

$$
d_{a}^{*}=-\star d_{a} \star
$$

So we may calculate,

$$
\begin{aligned}
-\mathrm{d}_{\mathrm{a}}^{*} \mathrm{~b}= & \star \mathrm{d}_{\mathrm{a}} \star \mathrm{b}=\star \mathrm{d}_{\mathrm{a}}(\mathrm{dt} \wedge \mathrm{b}) \\
= & \star\left(\mathrm{dt} \wedge\left[\nabla_{1}^{\mathrm{a}} \mathrm{b}_{1} \mathrm{~d} x^{134}+\nabla_{2}^{\mathrm{a}} \mathrm{b}_{1} \mathrm{~d} x^{234}+\nabla_{3}^{\mathrm{a}} \mathrm{b}_{1} \mathrm{~d} x^{312}+\nabla_{4}^{\mathrm{a}} \mathrm{b}_{1} \mathrm{~d} x^{412}\right]\right) \\
& +\star\left(\mathrm{dt} \wedge\left[-\nabla_{1}^{\mathrm{a}} \mathrm{b}_{2} \mathrm{~d} x^{124}+\nabla_{2}^{\mathrm{a}} \mathrm{b}_{2} \mathrm{~d} x^{213}-\nabla_{3}^{\mathrm{a}} \mathrm{b}_{2} \mathrm{~d} x^{324}+\nabla_{4}^{\mathrm{a}} \mathrm{b}_{2} \mathrm{~d} x^{413}\right]\right) \\
& +\star\left(\mathrm{dt} \wedge\left[\nabla_{1}^{\mathrm{a}} \mathrm{b}_{3} \mathrm{~d} x^{123}+\nabla_{2}^{\mathrm{a}} \mathrm{b}_{3} \mathrm{~d} x^{214}+\nabla_{3}^{\mathrm{a}} \mathrm{b}_{3} \mathrm{~d} x^{314}+\nabla_{4}^{\mathrm{a}} \mathrm{b}_{3} \mathrm{~d} x^{423}\right]\right) \\
= & \star{ }_{4}\left[\left(\nabla_{3}^{\mathrm{a}} \mathrm{b}_{1}-\nabla_{2}^{\mathrm{a}} \mathrm{b}_{2}+\nabla_{1}^{\mathrm{a}} \mathrm{b}_{3}\right) \mathrm{d} x^{123}+\left(\nabla_{2}^{\mathrm{a}} \mathrm{b}_{1}+\nabla_{3}^{\mathrm{a}} \mathrm{b}_{2}+\nabla_{4}^{\mathrm{a}} \mathrm{b}_{3}\right) \mathrm{d} x^{234}\right. \\
& \left.+\left(\nabla_{1}^{\mathrm{a}} \mathrm{b}_{1}+\nabla_{4}^{\mathrm{a}} \mathrm{b}_{2}-\nabla_{3}^{\mathrm{a}} \mathrm{b}_{3}\right) \mathrm{d} x^{341}+\left(\nabla_{4}^{\mathrm{a}} \mathrm{b}_{1}-\nabla_{1}^{\mathrm{a}} \mathrm{b}_{2}-\nabla_{2}^{\mathrm{a}} \mathrm{b}_{3}\right) \mathrm{d} x^{412}\right] \\
= & -\left(\nabla_{2}^{\mathrm{a}} \mathrm{b}_{1}+\nabla_{3}^{\mathrm{a}} \mathrm{b}_{2}+\nabla_{4}^{\mathrm{a}} \mathrm{b}_{3}\right) \mathrm{d} x^{1}+\left(\nabla_{1}^{\mathrm{a}} \mathrm{b}_{1}+\nabla_{4}^{\mathrm{a}} \mathrm{b}_{2}-\nabla_{3}^{\mathrm{a}} \mathrm{b}_{3}\right) \mathrm{d} x^{2} \\
& -\left(\nabla_{4}^{\mathrm{a}} \mathrm{b}_{1}-\nabla_{1}^{\mathrm{a}} \mathrm{b}_{2}-\nabla_{2}^{\mathrm{a}} \mathrm{b}_{3}\right) \mathrm{d} x^{3}+\left(\nabla_{3}^{\mathrm{a}} \mathrm{b}_{1}-\nabla_{2}^{\mathrm{a}} \mathrm{b}_{2}+\nabla_{1}^{\mathrm{a}} \mathrm{b}_{3}\right) \mathrm{d} x^{4}
\end{aligned}
$$

So we see that

$$
\left(\mathrm{d}_{+}^{\mathrm{a}}\right)^{*} \mathrm{~b}+\mathrm{d}_{\mathrm{a}} \mathrm{c}=\left(\begin{array}{c}
\nabla_{1}^{\mathrm{a}} \mathfrak{u}_{0}+\nabla_{2}^{\mathrm{a}} \mathrm{u}_{1}+\nabla_{3}^{\mathrm{a}} \mathrm{u}_{2}+\nabla_{4}^{\mathrm{a}} \mathfrak{u}_{3} \\
\nabla_{2}^{\mathrm{a}} \mathfrak{u}_{0}-\nabla_{1}^{\mathrm{a}} \mathfrak{u}_{1}-\nabla_{4}^{\mathrm{a}} \mathfrak{u}_{2}+\nabla_{3}^{\mathrm{a}} \mathfrak{u}_{3} \\
\nabla_{3}^{\mathrm{a}} \mathfrak{u}_{0}+\nabla_{4}^{\mathrm{a}} \mathfrak{u}_{1}-\nabla_{1}^{\mathrm{a}} \mathfrak{u}_{2}-\nabla_{2}^{\mathrm{a}} \mathrm{u}_{3} \\
\nabla_{4}^{\mathrm{a}} \mathfrak{u}_{0}-\nabla_{3}^{\mathrm{a}} \mathfrak{u}_{1}+\nabla_{2}^{\mathrm{a}} \mathfrak{u}_{2}-\nabla_{1}^{\mathrm{a}} \mathfrak{u}_{3}
\end{array}\right)
$$

Using the formulas from Lemma 6.21. we know that

$$
D_{j}^{a} u=\partial_{j} u+K_{u}^{a_{j}}=\partial_{j} u+\left[a_{j}, u\right]-\left(\begin{array}{c}
\dot{a}_{j} \\
0 \\
0 \\
0
\end{array}\right)=\nabla_{j}^{a} u-\left(\begin{array}{c}
\dot{a}_{j} \\
0 \\
0 \\
0
\end{array}\right),
$$

hence

$$
\left(d_{+}^{a}\right)^{*} b+d_{a} c-\dot{a}=D_{4}^{a} u-D_{5}^{a} u-J D_{6}^{a} u-K D_{7}^{a} u,
$$

for a left - left structure, where we multiplied the last three equations in (8.98) with -1 .

Theorem 8.31. If we use the octonionic structure given by $-\varphi^{\prime}$ (compare Definition 2.24), we get the same theorem but with $\mathrm{W}_{\mathrm{N}}^{\mathrm{s}}$ as target instead of $\mathrm{W}_{\mathrm{N}}$.

Proof. $\phi^{\prime} \rightarrow-\phi^{\prime}$ can be realized by inverting the coordinates $e^{1}, e^{2}$ and $e^{3}$ of the $\mathbf{G}_{\mathbf{2}}{ }^{-}$ structure. This changes the orientation of $\Lambda_{+}^{2}$, which implies that $\sigma(b, b)$ gets multiplied by -1 . Comparing with the last proof, we see that this gives the equations

\footnotetext{
${ }^{1}$ See also the footnote of Theorem 8.50
} 
$F_{+}^{a}=\mu_{N}^{s} \circ u$. The change of orientation also removes the "-" from equation (8.89), so that the second equation is equivalent to

$$
\left(d_{+}^{a}\right)^{*} b+d_{a} c=\left(\begin{array}{c}
\nabla_{1}^{a} u_{0}-\nabla_{2}^{a} u_{1}-\nabla_{3}^{a} u_{2}-\nabla_{4}^{a} u_{3} \\
\nabla_{2}^{a} u_{0}+\nabla_{1}^{a} u_{1}+\nabla_{4}^{a} u_{2}-\nabla_{3}^{a} u_{3} \\
\nabla_{3}^{a} u_{0}-\nabla_{4}^{a} u_{1}+\nabla_{1}^{a} u_{2}+\nabla_{2}^{a} u_{3} \\
\nabla_{4}^{a} u_{0}+\nabla_{3}^{a} u_{1}-\nabla_{2}^{a} u_{2}+\nabla_{1}^{a} u_{3}
\end{array}\right)
$$

which are the correct signs for the left - right structure of generalized Seiberg-Witten with target $W_{N}^{s}$ (without multiplying the equations by -1 ).

Remark 8.32. Note that the action of $\mathbf{S O}(3)$ is exactly the rotating action on $W_{\mathrm{N}}$ (compare to $(6.40)$ ). Once we include the boundary conditions, we equip $W_{N}$ with the action of (6.44), which also agrees with the given action, up to gauging by an element. Remark 8.33. This theorem works in both ways provided we start with some bundle $\mathrm{P} \rightarrow \mathrm{M}=\mathrm{I} \times \mathrm{X}$. Under certain conditions we can use a solution to the generalized Seiberg-Witten equations to induce a bundle on $M$.

Lemma 8.34. Let $(\mathrm{a}, \mathrm{u})$ be a solution to generalized Seiberg-Witten with target $\mathrm{W}_{\mathrm{N}}$ and principal bundle $\mathrm{P}^{\prime} \rightarrow \mathrm{X}$. If there is a reduction $\mathrm{P}_{\mathrm{X}} \rightarrow \mathrm{P}^{\prime}$ to a principal $\mathrm{G}-$ bundle then the solution induces a solution of the Haydys-Witten equations on the bundle $\mathrm{pr}_{\mathrm{X}}^{*} \mathrm{P}_{\mathrm{X}} \rightarrow \mathrm{M}$.

Proof. This follows immediately from the proof of the theorem. Note that the existence of the reduction is the only obstruction of transforming the bundle $\mathrm{P}^{\prime}$ to $\mathrm{P}$.

There are two interesting settings when such a reduction is given. For a more discussions on this see also section 9.2

Lemma 8.35. Such a reduction to a principal G-bundle are given if

- The bundle $\mathrm{P}^{\prime}$ is trivial,

- The aholomorphic spinor $\mathrm{u}$ takes values in $\mu_{\mathrm{N}}^{(s)}{ }^{-1}(0) \subset \mathrm{W}_{\mathrm{N}}$.

Proof. The first example is trivial, whereas the second uses the different possible descriptions of the Nahm equations. As explained in Remark 6.6, there are two different ways of describing the moduli space of Nahm equations as quotients. The description with the structure group $G$ is a reduction of the description with structure group $\mathfrak{C}^{\infty}(\mathrm{I}, \mathrm{G})$. The $\mathcal{G}$-equivariant map $u: \mathrm{P}_{+} \times_{X} \mathrm{P}^{\prime} \rightarrow W_{N}$ (or, since there is no action of SO $(3)$ on $W_{N}$ here $\left.u: P^{\prime} \rightarrow W_{N}\right)$ induces a map from $Q=P_{+}$to the associated bundle of $W_{N}$. We can use this map to pull back the reduction to the structure group $G$ back and have a reduction of $\hat{\mathrm{P}}^{\prime}$ to a principal G-bundle on $\mathrm{Q}$. Note that one has to be careful with the boundary conditions for this reduction, we have to allow different irreducible representations for the different ends of the interval. 
Notation 8.36. Denote by $\mathcal{M}_{\mathrm{HW}}^{\mathrm{P}}$ the moduli space of solutions of the Haydys-Witten equations on a principal bundle $P$. Denote by $\mathcal{M}_{g}^{\hat{P}^{\prime}, W_{N}}$ the moduli space of solutions to the generalized Seiberg-Witten equations on the bundle $\hat{P}^{\prime}$ with target $W_{N}$ (see e.g. [Pido4]).

Proposition 8.37. In the setting of this section,

$$
\mathcal{M}_{\mathrm{HW}}^{\mathrm{P}} \cong \mathcal{M}_{g}^{\hat{P}^{\prime} S^{\prime} W_{\mathrm{N}}} .
$$

Proof. In Proposition 8.28 it was shown the identification respects gauge equivalence classes. Note that $\mathfrak{C}^{\infty}\left(\hat{\mathrm{P}}^{\prime}, \mathcal{G}\right)^{\mathfrak{G} \times \mathrm{H}}$ is the gauge group of the given generalized Seiberg Witten system (see [Calio, Lemma 3.1.12]).

Theorem 8.38. In the setting of this section let $\mathrm{G}=\mathbf{U}(\mathrm{k})$ and let $(\mathrm{A}, \mathrm{B})$ be a solution of the Haydys-Witten equations on $\mathrm{P}$. We say that $(\mathrm{A}, \mathrm{B})$ satisfies the boundary conditions of type $k \in \mathbb{N}$, if

1. $\left\|\mathrm{l}_{\mathrm{I}} \hat{\mathrm{A}}\right\|$ is bounded when $\mathrm{t} \rightarrow \pm 1$ ( $\mathrm{t}$ is the coordinate of $\mathrm{I}$ ) for the pull back of $\mathrm{A}$ to $\hat{\mathrm{P}} \cong \hat{\mathrm{P}}_{\mathrm{X}} \times \mathrm{I}$ (everywhere on $\hat{\mathrm{P}}_{\mathrm{X}}$ )

2. $\|(t-1) \hat{B}-\rho\| \in \mathcal{O}(t-1)$ for $t \rightarrow 1$, where $\hat{B} \in \mathfrak{C}^{\infty}\left(\hat{P}, \Lambda_{+}^{2} \vee \otimes g\right)$ is the pull back of $\mathrm{B}$ and $\rho \in \Gamma\left(\operatorname{Hom}\left(\Lambda_{+}^{2}, \mathrm{Ad} \mathfrak{g}\right)\right)$ is induced by an irreducible representation $\Lambda_{+}^{2 \vee} \otimes \mathfrak{g} \cong$ $\mathfrak{s u}(2)^{\vee} \otimes \mathfrak{g}$ of $\mathfrak{s u}(2)$.

3. Similarly $\|(t+1) \hat{B}-\rho\| \in \mathcal{O}(t+1)$ for $t \rightarrow-1$.

Then these boundary conditions are invariant under the reduced gauge group where we require that $\mathrm{g} \in \mathcal{G}(\mathrm{P})=\mathfrak{C}^{\infty}(\mathrm{P}, \mathrm{G})^{\mathrm{G}}$ is such that the pull back $\hat{\mathrm{g}}$ to $\hat{\mathrm{P}}$ satisfies $\|\mathrm{g}-1\| \in \mathcal{O}(\mathrm{t}-1)$ for $\mathrm{t} \rightarrow 1$ and $\|\mathrm{g}-1\| \in \mathcal{O}(\mathrm{t}+1)$ for $\mathrm{t} \rightarrow-1$. Then the moduli space of solutions to the Haydys Witten equations subject to the boundary conditions of type $k \in N$ gauged by the reduced gauge group is isomorphic to the solutions of the generalized Seiberg-Witten equations with target $\mathrm{F}_{\mathrm{N}}^{\mathrm{k}}$,

$$
\mathcal{M}_{\mathrm{H} W}^{\mathrm{k}} \cong \mathcal{M}_{\mathrm{g} S W}^{\hat{\mathrm{P}}^{\prime}, \mathrm{F}_{\mathrm{N}}^{\mathrm{k}}}
$$

Proof. Note that we have chosen the boundary conditions so that the boundary conditions of the Nahm equations are satisfied. It remains to remark that the gauge group $9_{0}$ of the Nahm equations can be achieved by Remark 8.26. where we can pick $f$ to be (e.g.) $-x^{2}+1$.

Theorem 8.39. Restrict $\mathcal{M}_{\mathrm{H} W}^{\mathrm{k}}$ to solutions such that A has no self-dual curvature, i.e. $\mathrm{F}_{+}^{\mathrm{A}}=0$, and call that space $\mathcal{M}_{\mathrm{HW}, \mathrm{r}}^{\mathrm{k}}$. Then

$$
\mathcal{M}_{\mathrm{HW}, \mathrm{r}}^{\mathrm{k}} \cong\left\{\mathrm{u}: \mathrm{P}_{+} \rightarrow \mathcal{N}_{\mathrm{k}} \mid \mathrm{u} \text { is } \mathbf{S O}(3) \text {-equivariant and aholomorphic }\right\}
$$


Proof. Note that the requirement on the curvature implies that the reduced SeibergWitten equations are satisfied, i.e.

$$
\mathscr{D}_{\mathrm{a}} u=0 \quad \text { and } \quad \mu \circ u=0 .
$$

Noticing that the image is now the total space of a hyperkähler reduction bundle, we can identify the maps by Lemma 8.8, and Haydys [Hay12, Proposition 4.5] has shown that solutions of these are in correspondence with aholomorphic maps to the quotient.

Remark 8.40. The last theorem implies that $u: \hat{P}^{\prime} \rightarrow \mu^{-1}(0)$ carries the complete information of the solution to the generalized Seiberg-Witten equations. We can recover $a$ as follows. First we note that $u$ induces a $\mathbf{S O}(3)$-equivariant $\check{u}: P_{+} \rightarrow \mathcal{N}$ so that $P_{+} \cong \check{u}^{*} \mu^{-1}(0)$. The unique connection that satisfies the equations is then given by (the negative of) the pull back of the connection induced by the Riemannian submersion $\mu^{-1}(0) \rightarrow \mathcal{N}$. Alternatively we can use Lemma 2.13 to see that the pulled back connection is indeed anti self-dual, because the curvature of the connection of a hyperkähler reduction is of type $(1,1)$ with respect to all complex structures (compare chapter 2).

Theorem 8.41. Assume that $\mathrm{u}: \mathrm{P}_{+} \rightarrow \mathcal{N}_{k}$ is a $\mathbf{S O}(3)$-equivariant map. Then there is a bundle $\hat{\mathrm{P}}^{\prime} \rightarrow \mathrm{P}_{+}$and a connection a on it such that $(\mathrm{a}, \mathrm{u})$ satisfy the reduced generalized SeibergWitten equations. Furthermore, if there is a reduction $\hat{\mathrm{P}} \rightarrow \hat{\mathrm{P}}^{\prime}$ to the structure group $\mathrm{G}$, then this induces a solution of the Haydys Witten equations on the induced bundle $\mathrm{P} \rightarrow \mathrm{X} \times \mathrm{I}$ without self-dual curvature.

Proof. This follows from the last Remark and Lemma 8.34

Remark 8.42. There is a stronger statement than [Hay12, Proposition 4.5]. The connection exists for all $u: \hat{P}_{X} \rightarrow F_{N}$ given that a certain equations (which specializes to $\mathscr{D} \check{\mathrm{u}}=0$ on $\left.\mu^{-1}(0)\right)$ is satisfied and $\mu \circ u$ is small enough [|Pidi7|. It can also be shown that the connection if the image of $u$ meets no points which have a nontrivial $\mathcal{G}_{0}$ stabilizer. For this case it suffices to know $u$ in order to reconstruct the solution $(a, u)$. This is the justification for only transforming $u$ in our construction.

\subsubsection{Construction of Examples}

We can use this to construct classes of examples of solutions to the Haydys Witten equations.

Example 8.43. Let $X$ be a 4-dimensional manifold. Then every $u: P_{+} \rightarrow \mathcal{N}_{k}, \mathbf{S O}(3)$ equivariant and aholomorphic such that a reduction (as in Lemma 8.34) exists induces a solution of the Haydys Witten equations. If $P_{+}$is trivial (i.e. $X$ has a not necessarily integrable hyperkähler structure, compare to Proposition 2.44 then every aholomorphic map $X \rightarrow \mathcal{N}_{k}$ induces a solution of the Haydys-Witten equations. 
Example 8.44. Given any 4-dimensional manifold $X$, the map $u: P_{+} \rightarrow \mathcal{N}_{k}$ given by $\mathrm{u}=$ const $_{\mathrm{BPS}}$, the constant map to the unique spherical symmetric solution (of fixed mass 1 ), known as the BPS-monopole (given by $T_{i}=0$ for all $0 \leqslant i \leqslant 3$ ) [Pra75].

Example 8.45. If $X$ is a quaternionic 4-manifold, then any constant map $X \rightarrow \mathcal{N}_{k}$ produces an example.

Instead of putting the spinor first, we can also start with the connection.

Example 8.46. Given any $a \in \mathfrak{C}^{\infty}\left(I, \mathcal{C}\left(\hat{P}_{X}\right)\right)$ such that for every $t \in I F_{+}^{a(t)}=0$, i.e. $a(t)$ is a self-dual connection, and a $u \in \mathfrak{C}^{\infty}\left(\hat{\mathrm{P}}^{\prime}, \mu^{-1}(0)\right)$ with $\mathscr{D}_{\tilde{a}} \mathfrak{u}=0$, where $\tilde{a}$ is the connection associated via Proposition 8.24. Then this data induces a solution to the Haydys-Witten equations.

Example 8.47. As an example of the last class, take a to be the constant map to a anti self-dual connection, and $u$ ã-aholomorphic.

\subsection{3 $\quad \mathrm{G}_{2}$ Monopoles}

Let $M$ be a 7 manifold with (not necessarily integrable) $\mathbf{G}_{\mathbf{2}}$-structure $\varphi$ and $\psi$ the associated and coassociated form respectively $\left(\psi=\star_{7} \varphi\right)$ and $\mathrm{P} \rightarrow M$ a principal Gbundle. Assume further that $(A, \phi) \in \mathcal{C}(P) \times \Gamma(\operatorname{Ad}(P))$ is a solution of the $\mathbf{G}_{\mathbf{2}}$-monopole equations, i.e.

$$
\psi \wedge F^{A}=\star d_{A} \phi .
$$

For the first step assume that $M$ has a (not necessarily integrable) $\mathbf{S O}(4) \subset \mathbf{G}_{2}$ structure.

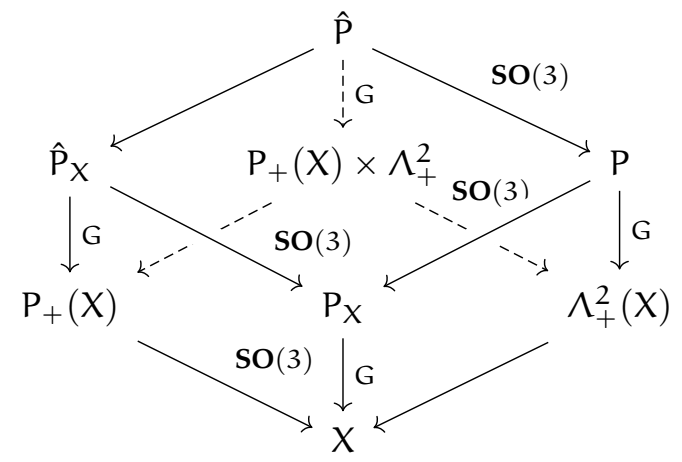

Figure 8.2: Involved principal bundles for $\mathbf{G}_{\mathbf{2}}$-Monopoles

Lemma 8.48. The $\mathbf{G}_{\mathbf{2}}$ monopole equations split to the following two sets of equations, given 
with respect to a frame in the reduced $\mathbf{S O}(4)$-frame bundle,

$$
\begin{aligned}
F_{23}^{A}-F_{45}^{A}-F_{67}^{A} & =\nabla_{1}^{A} \phi, \\
-F_{13}^{A}-F_{46}^{A}+F_{57}^{A} & =\nabla_{2}^{A} \phi, \\
F_{12}^{A}-F_{47}^{A}-F_{56}^{A} & =\nabla_{3}^{A} \phi,
\end{aligned}
$$

and

$$
\begin{aligned}
\mathrm{F}_{15}^{\mathrm{A}}+\mathrm{F}_{26}^{\mathrm{A}}+\mathrm{F}_{37}^{\mathrm{A}} & =\nabla_{4}^{\mathrm{A}} \phi, \\
-\mathrm{F}_{14}^{\mathrm{A}}-\mathrm{F}_{27}^{\mathrm{A}}+\mathrm{F}_{36}^{\mathrm{A}} & =\nabla_{5}^{\mathrm{A}} \phi, \\
\mathrm{F}_{17}^{\mathrm{A}}-\mathrm{F}_{24}^{\mathrm{A}}-\mathrm{F}_{35}^{\mathrm{A}} & =\nabla_{6}^{\mathrm{A}} \phi, \\
-\mathrm{F}_{16}^{\mathrm{A}}+\mathrm{F}_{25}^{\mathrm{A}}-\mathrm{F}_{34}^{\mathrm{A}} & =\nabla_{7}^{\mathrm{A}} \phi .
\end{aligned}
$$

Proof. Recall that the defining form of the $\mathbf{G}_{2}$ structure is given by

$$
\varphi^{\prime}=e^{123}-e^{145}-e^{167}-e^{246}+e^{257}-e^{347}-e^{356} .
$$

Now the reduction to $\mathbf{S O}(4)$ restricts to frames given by frames on $X$ which are lifted to frames on $\Lambda_{+}^{2} X$. By reordering, we may assume that $e_{1}, e_{2}$ and $e_{3}$ span the fiber of the vector bundle, whereas $e_{4}$ through $e_{7}$ span the base. This agrees with the associative form above.

The reduction $\mathbf{S O}(4) \hookrightarrow \mathbf{G}_{\mathbf{2}}$ splits the Grassmann bundles of $\mathrm{M}$ further. We arrive at the representations

\begin{tabular}{c|c|c} 
Space & $\mathbf{G}_{\mathbf{2}}$-representation & $\mathbf{S O}(4)$-representation \\
\hline$\Lambda^{1}$ & $\Lambda_{7}^{1}$ & $S^{20} \oplus \mathrm{S}^{11}$ \\
$\Lambda^{2}$ & $\Lambda_{7}^{2} \oplus \Lambda_{14}^{2}$ & $\left(S^{20} \oplus \mathrm{S}^{11}\right) \oplus\left(\mathfrak{s o}(4) \oplus \mathrm{S}^{31}\right)$ \\
$\Lambda^{3}$ & $\Lambda_{1}^{3} \oplus \Lambda_{7}^{3} \oplus \Lambda_{27}^{3}$ & $\left(S^{00}\right) \oplus\left(\mathrm{S}^{20} \oplus \mathrm{S}^{11}\right) \oplus\left(\mathrm{S}^{00} \oplus \mathrm{S}^{11} \oplus \mathrm{S}^{22} \oplus \mathrm{S}^{40} \oplus \mathrm{S}^{31}\right)$
\end{tabular}

To see this, note that the way $\mathbf{S O}(4) \subset \mathbf{G}_{\mathbf{2}}$ is embedded, immediately implies that $\mu \cong S^{11} \oplus S^{20}$. The rest of the claims is applying the Glebsch-Gordan decomposition to $S_{0}^{2} \mu$ and $\Lambda^{2} \mu$. Recall the definition of the $\mathbf{G}_{2}$-equivariant map

$$
\mathrm{G}(\psi): \wedge^{\bullet} \rightarrow \Lambda^{\bullet}, \quad \omega \mapsto \star(\psi \wedge \omega) .
$$

Then $\mathrm{G}(\psi)$ is in particular a $\mathbf{S O}(4)$-equivariant map and as such respects the decomposition into $\mathbf{S O}(4)$-representations, i.e. $G(\psi)$ identifies the $S^{20}$ and $S^{11}$ representations in $\Lambda^{1}$ and $\Lambda^{2}$ (note that $\mathfrak{s o}(4)$ also contains a $S^{20}$, however since $\beta$ is a $\mathbf{G}_{2}$ map, it's image is in $\Lambda_{7}^{2}$ when restricted to $\left.\Lambda^{1}\right)$.

We have arranged the frames such that $e^{1}, e^{2}$ and $e^{3}$ span $S^{20}$ and the other $e^{i}$ span $S^{11}$. Using the explicit form of $\varphi^{\prime}$ we can calculate how $\mathrm{G}(\psi)$ acts. The condition that $(A, \psi)$ is a $\mathbf{G}_{\mathbf{2}}$-monopole is equivalent to

$$
\mathrm{G}(\psi)\left(\mathrm{F}^{\mathrm{A}}\right)=\mathrm{d}_{\mathrm{A}} \varphi .
$$


If we write $F^{A}=\sum_{1 \leqslant i<j \leqslant 7} F_{i j}^{A} e^{i j}$, we arrive at the equations

$$
\begin{aligned}
F_{23}^{A}-F_{45}^{A}-F_{67}^{A} & =\nabla_{1}^{A} \phi \\
-F_{13}^{A}-F_{46}^{A}+F_{57}^{A} & =\nabla_{2}^{A} \phi \\
F_{12}^{A}-F_{47}^{A}-F_{56}^{A} & =\nabla_{3}^{A} \phi,
\end{aligned}
$$

for the $S^{20}$ representation and

$$
\begin{aligned}
F_{15}^{A}+F_{26}^{A}+F_{37}^{A} & =\nabla_{4}^{A} \phi \\
-F_{14}^{A}-F_{27}^{A}+F_{36}^{A} & =\nabla_{5}^{A} \phi \\
F_{17}^{A}-F_{24}^{A}-F_{35}^{A} & =\nabla_{6}^{A} \phi \\
-F_{16}^{A}+F_{25}^{A}-F_{34}^{A} & =\nabla_{7}^{A} \phi
\end{aligned}
$$

for the $S^{11}$ representation in this frame.

We can now, in order to pursue step 2, assume that $M \cong \Lambda_{+}^{2} X$ for some 4-manifold $X$. Using Proposition 8.18 to identify $A \in \mathcal{C}(P)$ with

$$
(a, c) \in\left[\mathfrak{C}^{\infty}\left(\Lambda_{+}^{2}, \mathcal{C}\left(\hat{P}_{X}\right)\right)^{\mathbf{S O}(3)} \times \Lambda_{+}^{2 \vee} \otimes \mathfrak{C}^{\infty}\left(\Lambda_{+}^{2}, \mathfrak{C}^{\infty}\left(\hat{\mathrm{P}}_{X}, \mathfrak{g}\right)^{\mathrm{G}}\right)^{\mathbf{S O}(3)}\right]_{\mathfrak{s o}(3)}
$$

and Proposition 8.15 to identify $\phi \in \Gamma(M, \operatorname{Ad}(P))$ with

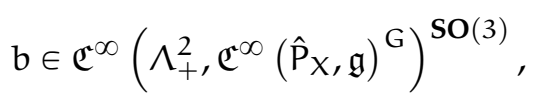

we arrive at the following equations.

Lemma 8.49. The $\mathbf{G}_{\mathbf{2}}$-monopole equations in differential equations form (compare to Remark (5.6) are given by

$$
\star \mathrm{F}^{\mathrm{c}}-\mathrm{d}^{\mathrm{c}} \mathrm{b}=\mathrm{F}_{+}^{\mathrm{a}}
$$

and

$$
\begin{aligned}
\partial_{1} a_{5}+\partial_{2} a_{6}+\partial_{3} a_{7} & =\nabla_{4}^{a} b+\nabla_{5}^{a} c_{1}+\nabla_{6}^{a} c_{2}+\nabla_{7}^{a} c_{3} \\
-\partial_{1} a_{4}-\partial_{2} a_{7}+\partial_{3} a_{6} & =\nabla_{5}^{a} b-\nabla_{4}^{a} c_{1}-\nabla_{7}^{a} c_{2}+\nabla_{6}^{a} c_{3} \\
\partial_{1} a_{7}-\partial_{2} a_{4}-\partial_{3} a_{5} & =\nabla_{6}^{a} b+\nabla_{7}^{a} c_{1}-\nabla_{4}^{a} c_{2}-\nabla_{5}^{a} c_{3} \\
-\partial_{1} a_{6}+\partial_{2} a_{5}-\partial_{3} a_{4} & =\nabla_{7}^{a} b-\nabla_{6}^{a} c_{1}+\nabla_{5}^{a} c_{2}-\nabla_{4}^{a} c_{3},
\end{aligned}
$$

where we interpret $\mathrm{c} \in \mathfrak{C}^{\infty}\left(\hat{\mathrm{P}}_{\mathrm{X}}, \Omega^{1}\left(\Lambda_{+}^{2}, \mathfrak{g}\right)\right)^{\mathrm{G} \times \mathbf{S O}(3)}$, as a connection on the trivial G-bundle on the fibers of $\Lambda_{+}^{2}$ and $\mathrm{b} \in \mathfrak{C}^{\infty}\left(\hat{\mathrm{P}}_{X}, \mathfrak{C}^{\infty}\left(\Lambda_{+}^{2}, \mathfrak{g}\right)\right)^{\mathrm{SO}(3) \times \mathrm{G}}$ as a section of the corresponding adjoint bundle. 
Proof. We have to choose local representatives of the connections. Let $\mathrm{s}: \mathrm{E} \supset \mathrm{U} \rightarrow \mathrm{P}$ be a local section of $P$. This is equivalent to a smooth map $U \rightarrow P_{X}$ that commutes with the projections $\mathrm{P}_{\mathrm{X}} \rightarrow \mathrm{X}$ and $\mathrm{E} \rightarrow \mathrm{X}$. Using $\mathrm{Q} \hookrightarrow \mathrm{Q} \times \mathrm{V} \rightarrow \mathrm{E}$, we can turn this map into a map from $\mathrm{Q} \supset \mathrm{pr}_{1}\left(\pi^{-1}(\mathrm{U})\right) \rightarrow \mathrm{PX}_{\mathrm{X}}$. A quick inspection shows that it commutes with the projections to $X$, hence it defines a section $\operatorname{pr}_{1}\left(\pi^{-1}(U)\right) \rightarrow \hat{P}_{X}$.

Tracing $A$ through the construction we see that if we lift the frame $e_{i}$ to the bundle $P$, then

$$
A\left(e^{i}\right)= \begin{cases}c_{i} & \text { if } i \in\{1,2,3\} \\ a_{i} & \text { if } i \in\{4,5,6,7\}\end{cases}
$$

This can be used to see that

$$
F_{i j}^{A}= \begin{cases}F_{i j}^{c} & \text { if } i, j \in\{1,2,3\} . \\ F_{i j}^{a} & \text { if } i, j \in\{4,5,6,7\} . \\ \partial_{i} a_{j}-\partial_{j} c_{i}+\left[c_{i}, a_{j}\right]=\partial_{i} a_{j}-\nabla_{j}^{a} c_{i} & \text { if } i \in\{1,2,3\}, j \in\{4,5,6,7\} .\end{cases}
$$

Using this, we identify the first three equations with

$$
\star \mathrm{F}^{\mathrm{c}}-\mathrm{F}_{+}^{\mathrm{a}}=\mathrm{d}^{\mathrm{c}} \mathrm{b} \quad \Leftrightarrow \quad \mu_{\mathrm{B}} \circ \mathrm{u}=\mathrm{F}_{+}^{\mathrm{a}},
$$

whereas the last four equations become

$$
\begin{aligned}
\partial_{1} a_{5}+\partial_{2} a_{6}+\partial_{3} a_{7}-\nabla_{5}^{a} c_{1}-\nabla_{6}^{a} c_{2}-\nabla_{7}^{a} c_{3} & =\nabla_{4}^{a} b \\
-\partial_{1} a_{4}-\partial_{2} a_{7}+\partial_{3} a_{6}+\nabla_{4}^{a} c_{1}+\nabla_{7}^{a} c_{2}-\nabla_{6}^{a} c_{3} & =\nabla_{5}^{a} b \\
\partial_{1} a_{7}-\partial_{2} a_{4}-\partial_{3} a_{5}-\nabla_{7}^{a} c_{1}+\nabla_{4}^{a} c_{2}+\nabla_{5}^{a} c_{3} & =\nabla_{6}^{a} b \\
-\partial_{1} a_{6}+\partial_{2} a_{5}-\partial_{3} a_{4}+\nabla_{6}^{a} c_{1}-\nabla_{5}^{a} c_{2}+\nabla_{4}^{a} c_{3} & =\nabla_{7}^{a} b
\end{aligned}
$$

In the last step we may use Proposition 8.24 to identify a with a connection on $\hat{\mathrm{P}}^{\prime}$,

$$
a \in \mathcal{C}\left(\hat{\mathrm{P}}^{\prime}\right)_{\mathfrak{s o}(3)}^{\mathbf{S O}(3)}
$$

For $\mathrm{b}$, we use Proposition 8.21 to identify $\mathrm{b}$ with an element in

$$
\mathfrak{C}^{\infty}\left(\hat{\mathrm{P}}^{\prime}, \operatorname{Lie}(\mathcal{G})\right)^{\mathcal{G} \rtimes \mathrm{SO}(3)} \cong \mathfrak{C}^{\infty}\left(\mathrm{P}_{+} \times{ }_{X} \mathrm{P}^{\prime}, \mathfrak{C}^{\infty}\left(\Lambda_{+}^{2}, \mathfrak{g}\right)\right)^{\mathcal{G} \rtimes \mathrm{SO}(3)}
$$

Similarly we may identify $\mathrm{c}$ with an element in

$$
\Lambda_{+}^{2} \otimes \mathfrak{C}^{\infty}\left(\mathrm{P}_{+} \times{ }_{x} \mathrm{P}^{\prime}, \mathfrak{C}^{\infty}\left(\Lambda_{+}^{2}, \mathfrak{g}\right)\right)^{\mathcal{G} \rtimes \mathrm{SO}(3)} .
$$

Putting this together, we get map

$$
\mathrm{u}=(\mathrm{b}, \mathrm{c}) \in \mathfrak{C}^{\infty}\left(\mathrm{P}_{+} \times_{X} \mathrm{P}^{\prime}, \mathbb{H} \otimes \mathfrak{C}^{\infty}\left(\Lambda_{+}^{2}, \mathfrak{g}\right)\right)^{\mathfrak{g} \rtimes \mathrm{SO}(3)},
$$

where we identified $\mathbb{H} \cong \mathbb{R} \otimes \Lambda_{+}^{2}$. 
Theorem 8.50. The pair $(A, \phi)$ is a $\mathbf{G}_{\mathbf{2}}$ monopole if and only if the associated pair $(a, u)$ is a solution to the generalized Seiberg Witten equations with target $\mathrm{W}_{\mathrm{B}}$, given by

$$
\mathscr{D}_{\mathrm{a}} \mathrm{u}=0, \quad \text { and } \quad \mu_{\mathrm{B}} \circ \mathrm{u}=\mathrm{F}_{+}^{\mathrm{a}},
$$

where $\mu_{\mathrm{B}}: \mathrm{W}_{\mathrm{B}} \rightarrow \mathrm{U}_{\mathrm{B}}$ is the moment map of the Bogomolny equations. The Dirac operator is defined via the Levi-Civita connection on $\mathrm{P}_{+}$and $\mathrm{a} \in \mathcal{C}\left(\hat{\mathrm{P}}^{\prime}\right)_{\mathfrak{s o}(3)}^{\mathbf{S O}(3)}$.

Proof. Finally we have to see that this is indeed the generalized Dirac operator acting on $u$. For this note that the action of $g \in \mathcal{G}$ on $\mathfrak{C}^{\infty}\left(\Lambda_{+}^{2}, g\right) \otimes \mathbb{H}$ if given by (compare Lemma 6.54).

$$
\frac{d(g \cdot A)}{d s}=[\xi, A]-d \xi, \quad \frac{d(g \cdot \varphi)}{d s}=[\xi, \varphi] .
$$

Taking a frame as before,

$$
D_{j}^{a} u=\partial_{j} u+K_{u}^{a_{j}}=\partial_{j} u+\left[a_{j}, u\right]-\left(\begin{array}{c}
0 \\
\partial_{1} a_{j} \\
\partial_{2} a_{j} \\
\partial_{3} a_{j}
\end{array}\right),
$$

so that the equations 8.127 through 8.130 become

$$
0=D_{4}^{a}\left(\begin{array}{c}
b \\
-c_{1} \\
-c_{2} \\
-c_{3}
\end{array}\right)+D_{5}^{a}\left(\begin{array}{c}
c_{1} \\
b \\
-c_{3} \\
c_{2}
\end{array}\right)+D_{6}^{a}\left(\begin{array}{c}
c_{2} \\
c_{3} \\
b \\
-c_{1}
\end{array}\right)+D_{7}^{a}\left(\begin{array}{c}
c_{3} \\
-c_{2} \\
c_{1} \\
b
\end{array}\right) .
$$

If we multiply the last three equations by -12 , we get

$$
0=\mathscr{D}_{\mathrm{a}} u=\mathrm{D}_{4}^{\mathrm{a}} \mathrm{u}-\mathrm{ID}_{5}^{\mathrm{a}} \mathrm{u}-\mathrm{JD}_{6}^{\mathrm{a}} \mathrm{u}-\mathrm{KD}_{7}^{\mathrm{a}} \mathrm{u},
$$

for a left - left structure, which agrees with Corollary 6.57,

Theorem 8.51. The pair $(A, \phi)$ is a $\mathbf{G}_{\mathbf{2}}$ monopole with $\mathbf{G}_{\mathbf{2}}$-structure given by $-\phi^{\prime}$ if and only if $(\mathrm{a}, \mathrm{u})$ is a solution to the generalized Seiberg Witten equations with target $\mathbb{H} \otimes$ $\mathfrak{C}^{\infty}\left(\Lambda_{+}^{2}, \mathfrak{g}\right)$, given by

$$
\mathscr{D}_{\mathrm{a}} \mathrm{u}=0, \quad \text { and } \quad \mu_{\mathrm{B}}^{\mathrm{s}} \circ \mathrm{u}=\mathrm{F}_{+}^{\mathrm{a}},
$$

where $\mu_{\mathrm{B}}^{\mathrm{s}}: \mathrm{W}_{\mathrm{B}} \rightarrow \mathrm{U}_{\mathrm{B}}$ is the moment map of self-dual Bogomolny monopoles. The dirac operator is defined via the Levi-Civita connection on $\mathrm{P}_{+}$and $\mathrm{a} \in \mathrm{C}\left(\hat{\mathrm{P}}^{\prime}\right)_{\mathfrak{s o}(3)}^{\mathrm{SO}(3)}$.

\footnotetext{
${ }^{2}$ This is an artifact of how we embedd $\mathbf{S O}(4)$ in $\mathbf{G}_{\mathbf{2}}$; see Remark 2.39
} 
Remark 8.52. Note that this agrees with how $\phi^{\prime}$ becomes $-\phi^{\prime}$, i.e. by mapping $e^{i} \rightarrow-e^{i}$, for $i \in\{1,2,3\}$. This changes the orientation of $\mathbb{R}^{3} \subset \mathbb{R}^{4}$, which turns the anti self-dual monopoles into self-dual monopoles.

The proof is similar, except that the last three equations need not be multiplied with -1 (for the Dirac operator). The signs agree with Corollary 6.57 to give a left right structure.

Just as for the Haydys-Witten equations, the only obstruction of reversing this construction is the existence of a reduction.

Lemma 8.53. Let $(a, u)$ be a solution to generalized Seiberg-Witten with target $W_{B}$ and principal bundle $\hat{\mathrm{P}}^{\prime} \rightarrow \mathrm{P}_{+}$. If there is a reduction $\hat{\mathrm{P}}_{\mathrm{X}} \rightarrow \hat{\mathrm{P}}^{\prime}$ to a principal $\mathrm{G}-$ bundle then the solution induces a solution of the $\mathbf{G}_{\mathbf{2}}$-Monopole equations on the bundle $\operatorname{pr}_{\mathrm{X}}^{*} \mathrm{P}_{\mathrm{X}} \rightarrow \mathrm{M}$.

See the discussion in section 9.2 about the existence of such reductions.

Proof. The proof is identical to the Haydys-Witten case, except that the bundle is an honest bundle on top of $\mathrm{P}_{+}$. Since the principal bundle of the hyperkähler reduction of the Bogomolny monopoles is $\mathbf{S O}(3)$-equivariant, and $u$ is $\mathbf{S O}(3)$-equivariant, the bundle $\hat{\mathrm{P}}^{\prime} \rightarrow \mathrm{P}_{+}$is $\mathbf{S O}(3)$ - equivariant. The reduction to $\hat{\mathrm{P}}_{X}$ is (because of our choice of embedding of $\mathrm{G} \rightarrow$ G) even $\mathbf{S O}(3)$-invariant, so corresponds to a bundle on the base $\mathrm{X}$.

Notation 8.54. Denote by $\mathcal{M}_{\mathbf{G}_{2}}^{P}$ the moduli space of $\mathbf{G}_{\mathbf{2}}$-instantons on a principal bundle P. Denote by $\mathcal{M}_{g}^{\hat{P}^{\prime}, W_{B}}$ the moduli space of solutions to the generalized Seiberg-Witten equations on the bundle $\hat{P}^{\prime}$ with target $W_{B}$ (see e.g. |Pido4|).

Proposition 8.55. In the setting of this section,

$$
\mathcal{M}_{\mathrm{G}_{2}}^{\mathrm{P}} \cong \mathcal{M}_{g}^{\hat{P}^{\prime}, W_{B}}
$$

Proof. The proof is identical to the case of the Haydys-Witten equations.

Theorem 8.56. In the setting of this section let $\mathrm{G}=\mathbf{S U}(2)$ and let $(A, \phi)$ be a solution of the $\mathbf{G}_{\mathbf{2}}$-monopole equations on $\mathrm{P}$. We say that $(A, \phi)$ satisfies the boundary conditions of type $k \in \mathbb{N}$, if for $r \rightarrow \infty$ (where $r$ denotes the radial distance to the zero section of $\Lambda_{+}^{2}$ )

1. $\|\mathrm{b}\|=1-\frac{\mathrm{k}}{2 \mathrm{r}}+\mathcal{O}\left(\mathrm{r}^{-2}\right)$

2. $d\|b\|=\mathcal{O}\left(r^{-2}\right)$

3. $\left\|\mathrm{d}_{\mathrm{c}} \mathrm{b}\right\|=\mathcal{O}\left(\mathrm{r}^{-2}\right)$,

where $\mathrm{c} \in \mathfrak{C}^{\infty}\left(\hat{\mathrm{P}}_{\mathrm{X}}, \Omega^{1}\left(\Lambda_{+}^{2}, \mathfrak{g}\right)\right)^{\mathbf{S O}(3) \times \mathrm{G}}$ and $\mathrm{b} \in \mathfrak{C}^{\infty}\left(\hat{\mathrm{P}}_{X}, \mathfrak{C}^{\infty}\left(\Lambda_{+}^{2}, \mathfrak{g}\right)\right)^{\mathbf{S O}(3) \times \mathrm{G}}$ is interpreted as in Lemma 8.49 Furthermore, we require that there is a $\mathbf{U}(1)$-bundle $\mathrm{R} \rightarrow \Lambda_{+}^{2}$ defined 
on the complement of some compact set on the fibers of $\wedge_{+}^{2}$ and with a fixed reduction of the Bogomolny data (compare to the boundary conditions of Bogomolny monopoles.

Then solutions to the $\mathbf{G}_{\mathbf{2}}$-monopole equations subject to the boundary conditions of type $k \in \mathbb{N}$ gauged by the reduced gauge group is isomorphic to the solutions of the generalized Seiberg-Witten equations with target $\mathrm{F}_{\mathrm{B}}^{\mathrm{k}}$,

$$
\mathcal{M}_{\mathbf{G}_{2}}^{k} \cong \mathcal{M}_{\mathrm{g} S W}^{\hat{P}^{\prime}, F_{\mathrm{B}}^{\mathrm{k}}}
$$

Proof. Again we have chosen the boundary conditions so that the boundary conditions of the Bogomolny equations are satisfied. It remains to remark that the gauge group $\mathcal{G}_{0}$ of the Bogomolny equations can be achieved by Remark 8.26, where we can pick $f$ to be $\frac{1}{\|x\|}$ smoothed around 0 .

Theorem 8.57. Restrict $\mathcal{M}_{\mathbf{G}_{2}}^{k}$ to solutions such that the transformed data given by a $\in$ $\mathfrak{C}^{\infty}\left(\Lambda_{+}^{2}, \mathcal{C}\left(\hat{\mathrm{P}}_{X}\right)\right)^{\mathrm{H}}$ has no self-dual curvature, i.e. $\mathrm{F}_{+}^{\mathrm{a}(v)}=0$ for every $v \in \Lambda_{+}^{2}$, and call that space $\mathcal{M}_{\mathbf{G}_{2}, r}^{k}$. Then

$$
\mathcal{M}_{\mathbf{G}_{2}, \mathrm{r}}^{\mathrm{k}} \cong\left\{\mathrm{u}: \mathrm{P}_{+} \rightarrow \mathcal{N}_{\mathrm{k}} \mid \mathrm{u} \text { is aholomorphic }\right\}
$$

Proof. The proof is identical to the case of the Haydys Witten equations.

Remark 8.58. We like to find a better description of $\mathcal{M}_{\mathbf{G}_{2}, r}^{k}$. Notice that for a manifold with $\mathbf{S O}(4) \subset \mathbf{G}_{\mathbf{2}}$ holonomy, the bundle $T^{\vee} M$ decomposes into $S^{20} \oplus S^{11}$. We can compute

$$
\begin{aligned}
\Lambda^{2} T^{\vee} M & =\Lambda^{2} S^{20} \oplus S^{20} \otimes S^{11} \oplus \Lambda^{2} S^{11} \\
& =S^{20} \oplus\left(S^{13} \oplus S^{11}\right) \oplus \mathfrak{s o}(4),
\end{aligned}
$$

and compare this to

$$
\Lambda^{2} T^{\vee} M=\underbrace{\left(S^{20} \oplus S^{11}\right)}_{\mu} \oplus \underbrace{\left(\mathfrak{s o}(4) \oplus S^{31}\right)}_{\mathfrak{g}_{2}},
$$

arising from the decomposition of the $\mathbf{G}_{\mathbf{2}}$-representations. Then the identity map

$$
S^{20} \oplus\left(S^{13} \oplus S^{11}\right) \oplus \mathfrak{s o}(4) \rightarrow\left(S^{20} \oplus S^{11}\right) \oplus\left(\mathfrak{s o}(4) \oplus S^{31}\right),
$$

is given by

$$
(a, b, c, d, e) \rightarrow\left(\frac{1}{\sqrt{2}}(a-d), c, \frac{1}{\sqrt{2}}(a+d), e, b\right),
$$

with respect to these decompositions. To see this notice that by the Lemma of Schur it is only unclear how the $S^{20}$ are mapped $\left(\mathfrak{s o}(4) \cong S^{20} \oplus S^{02}\right)$.

The first $S^{20}$ in the source is given by $\Lambda^{2}\left\langle e^{1}, e^{2}, e^{3}\right\rangle=\left\langle e^{12}, e^{23}, e^{31}\right\rangle$, the second one by $\Lambda_{+}^{2}\left\langle e^{4}, e^{5}, e^{6}, e^{7}\right\rangle=\left\langle e^{45}+e^{67}, e^{46}-e^{57}, e^{47}+e^{56}\right\rangle$. In the target the first $S^{20}$ is given by

$$
\left\langle e^{23}-e^{45}-e^{67}, e^{31}-e^{46}+e^{57}, e^{12}-e^{47}-e^{56}\right\rangle,
$$


(compare to equations 8.117) through (8.119); this depends on the choice of $\mathbf{G}_{\mathbf{2}}$ structure) which explains the $a-d$ component. The other follows by completing $\frac{1}{\sqrt{2}}(1,-1)$ to a ONB of $\mathbb{R}^{2}$. So the condition on $F^{A} \in \Lambda^{2} T^{\vee} M$ is that

$$
\mathrm{F}_{7,3}^{\mathrm{A}}=-\mathrm{F}_{14,3+}^{\mathrm{A}}
$$

where 7,3 is the projection onto the $S^{20} \subset \mu$ component and $14,3+$ the projection onto $\mathrm{S}^{20} \subset \mathfrak{g}_{2}$.

Theorem 8.59. Assume that $\mathrm{u}: \mathrm{P}_{+} \rightarrow \mathcal{M}_{\mathrm{k}}$ is a $\mathbf{S O}(3)$-equivariant map. Then there is a bundle $\hat{\mathrm{P}}^{\prime} \rightarrow \mathrm{P}_{+}$and a connection a on it such that $(\mathrm{a}, \mathrm{u})$ satisfy the reduced generalized Seiberg-Witten equations. Furthermore, if there is a reduction $\hat{\mathrm{P}} \rightarrow \hat{\mathrm{P}}^{\prime}$ to the structure group $\mathrm{G}$, then this induces a solution of the $\mathbf{G}_{\mathbf{2}}$-monopole equations on the induced bundle $\mathrm{P} \rightarrow \Lambda_{+}^{2} \mathrm{X}$ without self-dual curvature.

Proof. The proof is identical to the case of Haydys-Witten equations. Note that the pulled back bundle is $\mathbf{S O}(3)$-equivariant.

\subsubsection{Construction of Examples}

Similar to the case of the Haydys Witten equations, we can construct examples from this.

Example 8.6o. Let $X$ be a 4-dimensional manifold. Then every $u: P_{+} \rightarrow \mathcal{M}_{k}, \mathbf{S O}(3)$ equivariant and aholomorphic such that a reduction exists induces a solution of the $\mathrm{G}_{2}$-monopole equations. If $\mathrm{P}_{+}$is trivial (i.e. $\mathrm{X}$ has a not necessarily integrable hyperkähler structure) then every aholomorphic map $X \rightarrow \mathcal{M}_{k}$ induces a solution of the $\mathbf{G}_{\mathbf{2}}$-monopole equations.

Example 8.61. Given any 4-dimensional manifold $\mathrm{X}$, the map $\mathrm{u}: \mathrm{P}_{+} \rightarrow \mathcal{M}_{\mathrm{k}}$ given by $\mathrm{u}=$ const $_{\mathrm{BPS}}$, the constant map to the unique spherical symmetric solution (of fixed mass 1), known as the BPS-monopole [Pra75].

Example 8.62. If $X$ is a quaternionic 4-manifold, then any constant map $X \rightarrow \mathcal{M}_{k}$ produces an example.

Instead of putting the spinor first, we can also start with the connection.

Example 8.63. Given any $\mathrm{a} \in \mathfrak{C}^{\infty}\left(\Lambda_{+}^{2}, \mathrm{e}\left(\hat{\mathrm{P}}_{\mathrm{X}}\right)_{\mathfrak{s o}(3)}\right)^{\mathrm{SO}(3)}$ such that for every $\mathrm{t} \in \mathrm{I} \mathrm{F}_{+}^{\mathrm{a}(\mathrm{t})}=0$, i.e. $a(t)$ is a self-dual connection, and a $\mathfrak{C}^{\infty}\left(\hat{\mathrm{P}}^{\prime}, \mu^{-1}(0)\right)$ with $\mathscr{D}_{\tilde{a}} u=0$, where $\tilde{a}$ is the connection associated via Proposition 8.24. Then this data induces a solution to the $\mathrm{G}_{\mathbf{2}}$-monopole equations.

Example 8.64. As an example of the last class, choose $\check{a} \in \mathcal{C}\left(\mathrm{P}_{\mathrm{X}}\right)$ and pull it back to $\hat{\mathrm{P}}_{X}$. Then this is a connection $a \in \mathcal{C}\left(\hat{\mathrm{P}}_{X}\right)_{\mathfrak{s o}(3)}^{\mathbf{S O}(3)}$ and hence we can use the constant map $\Lambda_{+}^{2} \rightarrow a$ as our connection. 


\subsection{Adiabatic scaling}

If we want to look at the general picture, i.e. the identification of the moduli spaces with generalized Seiberg-Witten with target $F_{B / N}$, then the question arises how we can make sure that our solutions are approximate solutions (in the sense of Definitions 7.1 and 7.2). The idea is to use adiabatic scaling, i.e. changing the metric on the underlying manifold $X$ by $g \mapsto \varepsilon^{-2} g$. Having a $\mathbf{S O}(4) \subset \mathbf{G}_{2}, \mathbf{S O}(4) \subset \mathbf{S O}(5)$ reduction then results in different conformal weights on the equations

$$
\mathscr{D}_{\mathrm{a}} \mathrm{u}^{\varepsilon}=0 \quad \mu \circ \mathrm{u}^{\varepsilon}=\varepsilon^{2} \mathrm{~F}_{\mathrm{a}}^{+} .
$$

Example 8.65. - For $\mathbf{G}_{2}$ monopoles we have $\mathbf{S O}(4) \subset \mathbf{G}_{2}$ which gives $\Lambda^{1} \cong \Lambda_{4}^{1} \oplus \Lambda_{3}^{1}$, which (being $S^{11}$ and $S^{20}$ ) have weights 1 and 2 .

Hence adiabatic scaling stretches $\Lambda_{3}^{1}$ doubly, so $\mathcal{M}_{\varepsilon}^{\mathrm{Bog}}$ is the new target.

- On $\mathbf{S O}(4) \subset \mathbf{S O}(5)$ we want the same phenomenon, so

$$
\Lambda_{4}^{1} \oplus \Lambda_{1}^{1}
$$

we set the scaling on $\Lambda_{1}^{1}$ to have weight 2 and then $\mathcal{M}_{\varepsilon}^{\mathrm{Nahm}}$ is the new target.

We have shown that the Nahm transform commutes with these scalings in Proposition $7 \cdot 38$

\subsection{A Transform Between $\mathrm{G}_{2}$-Monopoles and solutions of the Haydys-Witten Equations}

Theorem 8.66. Let $\mathrm{X}$ be a 4-dimensional Riemannian manifold. Then for any $\check{\mathrm{u}}: \mathrm{P}_{+} \rightarrow \mathcal{M}^{k}$ $\left(\check{u}: \mathrm{P}_{+} \rightarrow \mathcal{N}^{k}\right)$ we define a transformed spinor $\check{v}: \mathrm{P}_{+} \rightarrow \mathcal{M}^{k} \rightarrow \mathcal{N}^{k}\left(\check{v}: \mathrm{P}_{+} \rightarrow \mathcal{N}^{k} \rightarrow \mathcal{M}^{k}\right)$. Now restrict $\mathcal{M}_{\mathbf{G}_{2}, r}^{k}\left(\mathcal{M}_{\mathrm{HW}, \mathrm{r}}^{\mathrm{k}}\right)$ to spinors u such that

1. All ǔ: $\mathrm{P}_{+} \rightarrow \mathcal{M}\left(\check{\mathrm{u}}: \mathrm{P}_{+} \rightarrow \mathcal{N}\right)$ belong to a $(\mathbf{S O}(3)$-equivariant) homotopy class.

2. $\check{u}^{*} \mu^{-1}(0)$ has a reduction.

3. For the transformed maps $\check{v}, \breve{v}^{*} \mu^{-1}(0)$ has a reduction.

Notice that if there is a reduction for one of the $\breve{\mathrm{u}}$, then there is one for all of them, and similar with the $\check{v}$, since the transformation of a homotopy class remains a homotopy class. Then we have maps

$$
{ }^{[\check{u}]} \mathcal{M}_{\mathrm{HW}, \mathrm{r}}^{\mathrm{k}} \leftrightarrows{ }^{[\check{v}]} \mathcal{M}_{\mathbf{G}_{2}, r}^{\mathrm{k}} .
$$

These maps are inverses of each other, showing

$$
{ }^{[\check{u}]} \mathcal{M}_{\mathrm{HW}, \mathrm{r}}^{\mathrm{k}} \cong{ }^{[\check{v}]} \mathcal{M}_{\mathbf{G}_{2,}, \mathrm{r}}^{\mathrm{k}} .
$$


Proof. The claim follows from Theorem 8.39 and 8.57 together with Proposition 7.39 and the fact that the Nahm transform is - up to a constant factor - a hyperkähler isometry [Nak91. Theorem 6.5]. The latter ensures that a aholomorphic map gets mapped to a aholomorphic map. The former two theorems identify the solutions with equivariant maps $\mathrm{P}_{+} \rightarrow \mathcal{N}_{k} / \mathcal{N}_{k}$ and the Proposition ensures that the transformed data is still $\mathbf{S O}(3)$-equivariant.

The Nahm transform between $\mathcal{M}_{k} / \mathcal{N}_{k}$ is always injective, the injectivity can only break if there are multiple connections that make $\check{u}$ to a aholomorphic map. This is true if and only if $\check{u}$ meets a nondiscrete stabilizer of $\mathcal{G}_{0}$.

Example 8.67. Take $X$ a Riemannian 4-manifold. The homotopy class of the map $\mathrm{P}_{+} \rightarrow$ $\mathcal{M}_{k} / \mathcal{N}_{k}$ mapping to the BPS-monopole satisfies the requirements of the last theorem, because the pull back is trivial and the transformed class contains the BPS-monopole.

Example 8.68. If we let $X=\mathbb{R}^{4}$, then $\mathrm{P}_{+}$is trivial and hence any two maps $\check{\mathrm{u}}: \mathrm{P}_{+} \rightarrow$ $\mathcal{M}_{k} / \mathcal{N}_{k}$ are homotopic. Furthermore the reduction always exist and we get

$$
\mathcal{M}_{\mathrm{HW}, \mathrm{r}}^{\mathrm{k}} \cong \mathcal{M}_{\mathrm{G}_{2}, \mathrm{r}}^{\mathrm{k}}
$$

Example 8.69. If $X$ is a quaternionic 4-manfiold, then any aholomorphic map $X \rightarrow \mathcal{N}_{k}$ $\left(\mathcal{M}_{k}\right)$ such that the reduction exists gives riseto a transformed $X \rightarrow \mathcal{M}_{k}\left(\mathcal{N}_{k}\right)$. If the reduction exists, we get a transform as in the last example.

Theorem 8.70. Let $\mathrm{X}$ be a compact 4-dimensional Riemannian manifold and $\mathrm{P}_{\mathrm{X}} \rightarrow \mathrm{X}$ a principal $\mathbf{U}(\mathrm{k})$-bundle. Then there is a open neighborhood $\mu_{\mathrm{N}}^{-1}(0) \subset \mathrm{U} \subset \mathrm{F}_{\mathrm{N}}^{\mathrm{k}}$ such that we have a map

$$
u \in \mathfrak{C}^{\infty}\left(\mathrm{P}_{+} \times x \mathrm{P}^{\prime}, \mathrm{U}\right)^{\mathcal{G}_{0}^{N} \rtimes \mathbf{S O}(3)} \rightarrow v \in \mathfrak{C}^{\infty}\left(\mathrm{P}_{+} \times x \mathrm{R}_{[\tilde{v}]}^{\prime}, \mathrm{F}_{\mathrm{B}}^{\mathrm{k}}\right)^{\mathcal{G}_{0}^{\mathrm{B}} \rtimes \mathrm{SO}(3)},
$$

where $\mathrm{R}_{[\check{v}]}^{\prime}$ is the pull back of the principal bundle $\mathrm{F}_{\mathrm{N}}^{\mathrm{k}} \rightarrow \mathrm{F}_{\mathrm{N}}^{\mathrm{k}} / \mathcal{G}_{0}$, by the transformed projection $\check{v}$.

Proof. The action of $\mathcal{G}_{0}$ is free and proper on $\mathrm{F}_{\mathrm{N}}^{\mathrm{k}}$ because of the framing (see e.g. [MV81]). Given a $u: \hat{P}^{\prime} \rightarrow F_{N}^{k}$, we apply the generalized Nahm transform to define the transformed map $\check{v}: \mathrm{P}_{+} \rightarrow \mathrm{F}_{\mathrm{B}}^{\mathrm{k}} / \mathcal{G}_{0}$ and pull back the bundle $\mathrm{F}_{\mathrm{B}}^{\mathrm{k}}$ along it to define the transformed bundle.

Of course the map can also be formulated in the other direction with the very same ideas.

Theorem 8.71. Let $\mathrm{X}$ be a compact 4-dimensional Riemannian manifold and $\mathrm{P}_{\mathrm{X}} \rightarrow \mathrm{X}$ a principal $\mathbf{S U}(2)$-bundle. Then there is a open neighborhood $\mu_{\mathrm{B}}{ }^{-1}(0) \subset \mathrm{U} \subset \mathrm{F}_{\mathrm{B}}^{\mathrm{k}}$ such that we have a map

$$
u \in \mathfrak{C}^{\infty}\left(\mathrm{P}_{+} \times{ }_{x} \mathrm{P}^{\prime}, \mathrm{u}\right)^{\mathcal{G}_{0}^{\mathrm{B}} \rtimes \mathbf{S O}(3)} \rightarrow v \in \mathfrak{C}^{\infty}\left(\mathrm{P}_{+} \times_{x} \mathrm{R}_{[\check{v}]}^{\prime}, \mathrm{F}_{\mathrm{N}}^{\mathrm{k}}\right)^{\mathcal{G}_{0}^{N} \rtimes \mathbf{S O}(3)}
$$


where $\mathrm{R}_{[\check{v}]}^{\prime}$ is the pull back of the principal bundle $\mathrm{F}_{\mathrm{B}}^{\mathrm{k}} \rightarrow \mathrm{F}_{\mathrm{B}}^{\mathrm{k}} / \mathcal{G}_{0}$, by the transformed projection $\check{v}$.

Remark 8.72. The space $\mathrm{F}_{\mathrm{B}}^{\mathrm{k}}$ is contractible and as such $\mathrm{F}_{\mathrm{B}}^{\mathrm{k}} \rightarrow \mathrm{F}_{\mathrm{B}}^{\mathrm{k}} / \mathcal{G}_{0}$ is a classifying bundle for $\mathcal{G}_{0}$. However since we did not extend the Nahm transform to all of $\mathrm{F}_{\mathrm{B}}^{\mathrm{k}}$, not all maps $u$ are necessarily homotopic, so again we can have images in different bundles.

Remark 8.73. Notice that Theorem 8.70 and Theorem 8.71 give extensions of the maps defined in Theorem 8.66

Remark 8.74. The transform described in Theorem 8.70 and Theorem 8.71 also describes how the spinors are transformed in Theorem 8.66. Since the neighborhood $\mathrm{U}$ retracts to $\mu^{-1}(0)$, the involved bundles agree. As such it seems to be the natural extension of the transform. What remains to discuss is how the connections enter the transform. Since I have not arrived at a satisfactory answer for that, it will be discussed in the Outlook chapter.

Remark 8.75. The fact that we have found a transform of our topological data (the involved bundles) is rather remarkable, since the Nahm transform does not have such a feature, all involved bundles are trivial(lizable).

Remark 8.76. It may be tempting to try to use adiabatic scaling to make sure that the data is in the neighborhood $\mathrm{U}$; however, this also scales the Nahm data, so that $\mathscr{D}^{\varepsilon}=$ $\varepsilon \mathscr{D}$ (compare (7.171) ) hence $\left\|\mathrm{G}_{0}^{\varepsilon}\right\|=\frac{1}{\varepsilon^{2}}\left\|\mathrm{G}_{0}\right\|$ so that the defining condition (compare Corollary $7 \cdot 30$ and $7 \cdot 32$.

$$
\left\|\mathrm{cl}_{\mathrm{F}_{+}^{\mathrm{a}}}\right\|<\left\|\mathrm{G}_{\mathrm{O}}\right\|^{-1}
$$

remains unchanged under adiabatic scaling. It seems this should be fixable by finding another requirement as discussed in section 7.6. 


\section{Chapter 9}

\section{Outlook}

\subsection{About the Connections}

We have only explained how to transform the spinors in Theorem 8.70 and 8.71 and not described how to transform the connection. There is a formula [Pid17] guaranteeing the existence of a unique connection for any spinor $u$ with image sufficiently close to $\mu^{-1}(0)$ such that

$$
\mathscr{D}_{\mathrm{a}} \mathrm{u}=0 .
$$

This agrees with the theme that the spinor already carries all the information of the solution, as it is the case for spinors with values in $\mu^{-1}(0)$. However, this time we have to check by hand that the second equation of the generalized Seiberg-Witten equations are satisfied, i.e.

$$
\mu \circ \mathrm{u}=\mathrm{F}_{+}^{\mathrm{a}} .
$$

We have done a substantial step towards that goal by giving an explicit formula for the transformed error in Corollary 7.21 and 7.26. This gives an explicit map $\mathrm{U}_{\mathrm{N}} \leftrightarrows \mathrm{U}_{\mathrm{B}}$, which may be associated with the normal bundles of $\mu^{-1}(0) \subset F_{B / N}$ (see Proposition 3.16). This suggests that it might be best to first try to show that the linearized versions of Corollary 7.23 and 7.28 of the error map satisfies a linearized version of the second equations.

\subsection{About the Boundary Conditions and Other Remarks}

The boundary conditions we chose in this work were dictated by the fact that we wanted to use the Nahm transform. However it would be interesting to understand how the conditions in [MW13] translate to boundary conditions on the space of Nahm data. In particular it would be interesting to understand how the knot topology can be understood in this framework. 
While there is a blank check principal bundle reduction in the case of the Nahm equations in Lemma 8.35, the author is unaware of such a reduction for the case of Bogomolny equations.

It seems interesting to take a closer look at the topology of the involved bundles in the transform defined via Theorem 8.66 (and extended by Theorem 8.70 and 8.71 . In particular it would be interesting to find an explicit example for which the transformed data consists of multiple (different) bundles.

Another question that arose while working on this is if the Nahm transform can be lifted to dimensions 5 and 7 without the necessity of the intermediate step to generalized Seiberg-Witten. For this note that a 5-dimensional manifold with holonomy in $\mathbf{S O}(4)$ (even $\mathbf{O}(4)$ ) is spin, as is a 7-dimensional manifold with holonomy in $\mathbf{G}_{2}$. It is possible to copy the definitions to this setting, but many analytic questions would need addressing (the index, positivity, ... of the operators). Also the proof of the transform fails because of cross-terms in the general setting. This could maybe be addressed by requiring certain invariance of the involved data.

For the Examples 8.43 and 8.60 it seems interesting to understand the space of aholomorphic maps from a 4-dimensional quaternionic manifold to the spaces $\mathcal{M}_{N}$ and $\mathcal{M}_{\mathrm{B}}$.

It might also be interesting to look at the physical implications of the mentioned transform, even though the author's knowledge does not suffice to make an informed judgment. 


\section{Bibliography}

[AFG75] J. Arafune, P. G. O. Freund, and C. J. Goebel. "Topology of Higgs fields". In: J. Mathematical Phys. 16 (1975), pp. 433-437. ISSN: 0022-2488. uRL: https: //doi.org/10.1063/1.522518.

[AH88] Michael Atiyah and Nigel Hitchin. The geometry and dynamics of magnetic monopoles. M. B. Porter Lectures. Princeton University Press, Princeton, NJ, 1988, pp. viii+134. ISBN: o-691-08480-7. DOI: 10.1515/9781400859306. URL: http://dx.doi.org/10.1515/9781400859306.

[Bauog] H. Baum. Eichfeldtheorie: Eine Einführung in die Differentialgeometrie auf Faserbündeln. Springer Berlin Heidelberg, 2009.

[BC64] Richard L. Bishop and Richard J. Crittenden. Geometry of manifolds. Pure and applied mathematics. UKM. New York, London: Academic Press, 1964. ISBN: o-12-102450-4. URL: http://opac . inria.fr/record=b1079826.

[Bieo8] Roger Bielawski. "Curvature of hyperkaehler quotients". In: Cent. Eur. J. Math. 6 (2008), pp. 191-203.

[Bry87] Robert L. Bryant. "Metrics with Exceptional Holonomy". In: Annals of Mathematics 126 (3 Nov. 1987). DoI: $10.2307 / 1971360$.

[Calio] Martin Callies. "Dimensional reduction for the generalized Seiberg-Witten equations and the Chern-Simons-Dirac functional". MA thesis. Universität Göttingen, 2010.

[Cal78] Constantine Callias. "Axial anomalies and index theorems on open spaces". In: Comm. Math. Phys. 62.3 (1978), pp. 213-234. IssN: oo1o-3616. uRL: http: //projecteuclid.org/euclid.cmp/1103904395

[Don83] S. K. Donaldson. "An application of gauge theory to four-dimensional topology". In: J. Differential Geom. 18.2 (1983), pp. 279-315. ISSN: 0022-040X. URL: http://projecteuclid.org/euclid.jdg/1214437665.

[Don84] S. K. Donaldson. "Nahm's equations and the classification of monopoles". In: Communications in Mathematical Physics 96 (3 1984). 
[DSi1a] Simon Donaldson and Ed Segal. "Gauge theory in higher dimensions, II". In: Surveys in differential geometry. Volume XVI. Geometry of special holonomy and related topics. Vol. 16. Surv. Differ. Geom. Int. Press, Somerville, MA, 2011, pp. 1-41. URL: https://doi.org/10.4310/SDG.2011.v16.n1.a1

[DSi1b] Simon Donaldson and Ed Segal. "Gauge theory in higher dimensions, II". In: Surveys in differential geometry. Volume XVI. Geometry of special holonomy and related topics. Vol. 16. Surv. Differ. Geom. Int. Press, Somerville, MA, 2011, pp. 1-41. DOI: 10.4310/SDG.2011.v16.n1.a1. URL: http://dx.doi. org/10.4310/SDG.2011.v16.n1.a1

[Gaig9] Ana Rita Pires Gaio. "J-Holomorphic Curves and Moment Maps". PhD thesis. University of Warwick, 1999.

[GN92] Toru GOCHO and Hiraku NAKAJIMA. "Einstein-Hermitian connections on Hyper-Kähler quotients". In: Journal of the Mathematical Society of Japan 44.1 (Jan. 1992), pp. 43-51. DOI: 10.2969/jms j/04410043. URL: http://dx. doi.org/10.2969/jmsj/04410043.

[Halo3] Brian C. Hall. Lie Groups, Lie Algebras, and Representations: An Elementary Introduction. 1st ed. Graduate Texts in Mathematics 222. Springer-Verlag New York, 2003.

[Hay12] Andriy Haydys. "Gauge theory, calibrated geometry and harmonic spinors". In: J. Lond. Math. Soc. (2) 86.2 (2012), pp. 482-498. ISSN: 0024-6107. URL: https://doi.org/10.1112/jlms/jds008.

[Hay15a] Andriy Haydys. "Dirac operators in gauge theory". In: New ideas in low dimensional topology. Vol. 56. Ser. Knots Everything. World Sci. Publ., Hackensack, NJ, 2015, pp. 161-188. URL: https://doi.org/10.1142/9789814630627_ 0005 .

[Hay15b] Andriy Haydys. "Fukaya-Seidel category and gauge theory". In: J. Symplectic Geom. 13.1 (2015), pp. 151-207. ISSN: 1527-5256. URL: https://doi.org/ 10.4310/JSG.2015.v13.n1.a5.

[Hit83] N. J. Hitchin. "On the construction of monopoles". In: Comm. Math. Phys. 89.2 (1983), pp. 145-190. ISSN: 0010-3616. URL: http: // projecteuclid. org/euclid.cmp/1103922679.

[Hit+87] N. J. Hitchin et al. "Hyper-Kähler metrics and supersymmetry". In: Communications in Mathematical Physics 108.4 (1987), pp. 535-589. URL: http: //projecteuclid.org/euclid.cmp/1104116624

[Jaro4] Marcos Jardim. "A survey on Nahm transform". In: J. Geom. Phys. 52.3 (2004), pp. 313-327. ISSN: 0393-0440. URL: https ://doi .org/10.1016/j. geomphys.2004.03.006. 
[JT80] Arthur Jaffe and Clifford Taubes. Vortices and monopoles. Vol. 2. Progress in Physics. Structure of static gauge theories. Birkhäuser, Boston, Mass., 1980, pp. v+287. ISBN: 3-7643-3025-2.

[KM97] Andreas Kriegl and Peter W. Michor. The convenient setting of global analysis. Vol. 53. Mathematical Surveys and Monographs. American Mathematical Society, Providence, RI, 1997, pp. x+618. ISBN: o-8218-0780-3. DoI: 10.1090/ surv/053. URL: http://dx.doi.org/10.1090/surv/053.

[KN69] S. Kobayashi and K. Nomizu. Foundations of Differential Geometry: Vol.: 2. Interscience Tracts in Pure and Applied Mathematics. Interscience Publishers, 1969 .

[Max65] J. C. Maxwell. "A dynamical theory of the electromagnetic field". In: Phil. Trans. R. Soc. (London) 155 (Jan. 1865), pp. 459-512. DOI: $10.1098 /$ rstl. 1865.0008

[MV81] P. K. Mitter and C.-M. Viallet. "On the bundle of connections and the gauge orbit manifold in Yang-Mills theory". In: Comm. Math. Phys. 79.4 (1981), pp. 457-472. ISSN: oo10-3616. URL: http://projecteuclid.org/euclid. cmp/1103909137.

[MW13] Rafe Mazzeo and Edward Witten. The Nahm Pole Boundary Condition. 2013. eprint: arXiv:1311.3167.

[Nak91] Hiraku Nakajima. Monopoles and Nahm's Equations. Einstein Metrics and Yang-Mills Connections. Marcel Dekker, New York, 1991.

[Oli14] Goncalo Oliveira. "Monopoles on the Bryant-Salamon $\mathrm{G}_{2}$-manifolds". In: J. Geom. Phys. 86 (2014), pp. 599-632. ISSN: 0393-0440. URL: https ://doi . org/10.1016/j.geomphys.2014.10.005.

[Onio4] Arkady L. Onishchik. Lectures on real semisimple Lie algebras and their representations. ESI Lectures in Mathematics and Physics. European Mathematical Society (EMS), Zürich, 2004, pp. $x+86$. IsBN: 3-03719-002-7. DOI: 10.4171/002, URL: http://dx.doi.org/10.4171/002,

[O'N66] Barrett O'Neill. “The fundamental equations of a submersion." In: The Michigan Mathematical Journal 13.4 (Dec. 1966), pp. 459-469. Dor: $10.1307 /$ mmj/1028999604. URL: http://dx.doi.org/10.1307/mmj/1028999604.

[Pido4] Victor Pidstrygach. "Hyperkähler Manifolds and Seiberg-Witten Equations". In: Proceedings of the Steklov Institute of Mathematics 246 (2004), pp. 249-262.

[Pid17] Victor Pidstrygach. personal communication. Nov. 27, 2017.

[Pra75] Charles M. Prasad M. K.; Sommerfield. "Exact Classical Solution for the 't Hooft Monopole and the Julia-Zee Dyon". In: Physical Review Letters 35 (12 Sept. 1975). Dor: $10.1103 /$ PhysRevLett. 35.760 . 
[Sal89] Simon Salamon. Riemannian Geometry and Holonomy Groups. Subsequent. Research Notes in Mathematics Series. Longman Sc \& Tech, 1989.

[SW94a] N. Seiberg and E. Witten. "Electric-magnetic duality, monopole condensation, and confinement in $\mathrm{N}=2$ supersymmetric Yang-Mills theory". In: Nuclear Phys. B 426.1 (1994), pp. 19-52. ISSN: 0550-3213. URL: https : //doi.org/10.1016/0550-3213(94)90124-4.

[SW94b] N. Seiberg and E. Witten. “Erratum: "Electric-magnetic duality, monopole condensation, and confinement in $\mathrm{N}=2$ supersymmetric Yang-Mills theory"'". In: Nuclear Phys. B 430.2 (1994), pp. 485-486. ISSN: 0550-3213. URL: https://doi.org/10.1016/0550-3213(94)00449-8.

[Tau99] Clifford Henry Taubes. "Nonlinear generalizations of a 3-manifold's Dirac operator". In: Trends in mathematical physics (Knoxville, TN, 1998). Vol. 13. AMS/IP Stud. Adv. Math. Amer. Math. Soc., Providence, RI, 1999, pp. 475486.

[Varo1] V. S. Varadarajan. "Spin(7)-subgroups of $\mathrm{SO}(8)$ and Spin(8)". In: Expo. Math. 19.2 (2001), pp. 163-177. ISSN: 0723-0869. DOI: 10.1016 / S07230869 (01)80027-X. URL: http://dx.doi.org/10.1016/S0723-0869(01) $80027-\mathrm{X}$.

[Xuo8] Feng Xu. "Geometry of SU(3) Manifolds". PhD thesis. Duke University, 2008.

[Yoko9] I. Yokota. "Exceptional Lie groups". In: ArXiv e-prints (Feb. 2009). arXiv: 0902.0431 [math.DG]. 